NUREG/CR-5815

BNL-NUREG-52311

\title{
Evaluations of 1990 \\ PRISM Design Revisions
}

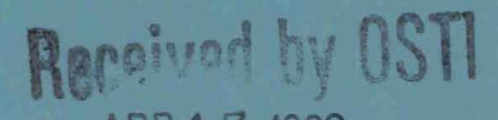

APR 171992

Prepared by

G. J. Van Tuyle, G. C. Slovik, B. C. Chan,

A. L. Aronson, R. J. Kennett

Brookhaven National Laboratory

Prepared for

U.S. Nuclear Regulatory Commission 


\section{AVAILABILITY NOTICE}

Availability of Reference Materials Cited in NRC Publications

Most documents cited in NRC publications will be avallable from one of the following sources:

1. The NRC Publlc Document Room, 2120 L Street, NW., Lower Level, Washington, DC 20555

2. The Superintendent of Documents, U.S. Government Printing Office, P.O. Box 37082 , Washington, DC 20013-7082

3. The National Technical Information Service, Springfield, VA 22161

Although the listing that follows represents the majority of documents cited in NRC publications, it is not Intended to be exhaustive.

Referenced documents avallable for inspection and copying for a fee from the NRC Public Document Room Include NRC correspondence and internal NRC memoranda; NRC bulletins, circulars, Information notices, Inspection and investigation notices; licensee event reports; vendor reports and correspondence; Commission papers; and applicant and llcensee documents and correspondence.

The following documents in the NUREG series are avallable for purchase from the GPO Sales Program: formal NRC staff and contractor reports, NRC-sponsored conference proceedings, and NRC booklets and brochures. Also available are regulatory guides, NRC regulations in the Code of Federal Regulations, and Nuclear Regulatory Commission Issuances.

Documents available from the National Technical Information Service include NUREG-series reports and technical reports prepared by other Federal agencies and reports prepared by the Atomic Energy Commission, forerunner agency to the Nuclear Regulatory Commission.

Documents available from public and special technical libraries include all open literature items, such as books, Journal articles, and transactions. Federal Register notices, Federal and State legislation, and congressional reports can usually be obtained from these libraries.

Documents such as theses, dissertations, foreign reports and translations, and non-NRC conference proceedings are available for purchase from the organization sponsoring the publication cited.

Single copies of NRC draft reports are avallable free, to the extent of supply, upon written request to the Office of Administration. Distribution and Mall Services Section, U.S. Nuclear Regulatory Commission, Washington, DC 20555.

Coples of industry codes and standards used in a substantive manner in the NRC regulatory process are maintained at the NRC Library, 7920 Norfolk Avenue, Bethesda, Maryland, for use by the public. Codes and standards are usually copyrighted and may be purchased from the originating organization or, If they are American National Standards, from the American National Standards Institute, 1430 Broadway, New York, NY 10018.

\section{DISCLAIMER NOTICE}

This report was prepared as an account of work sponsored by an agency of the United States Government. Neither the United States Government nor any agency thereof, or any of their employees, makes any warranty, expressed or implied, or assumes any legal liability of responsibility for any third party's use, or the results of such use, of any information, apparatus, product or process disclosed in this report, or represents that its use by such third party would not infringe privately owned rights. 


\section{DISCLAIMER}

This report was prepared as an account of work sponsored by an agency of the United States Government. Neither the United States Government nor any agency Thereof, nor any of their employees, makes any warranty, express or implied, or assumes any legal liability or responsibility for the accuracy, completeness, or usefulness of any information, apparatus, product, or process disclosed, or represents that its use would not infringe privately owned rights. Reference herein to any specific commercial product, process, or service by trade name, trademark, manufacturer, or otherwise does not necessarily constitute or imply its endorsement, recommendation, or favoring by the United States Government or any agency thereof. The views and opinions of authors expressed herein do not necessarily state or reflect those of the United States Government or any agency thereof. 


\section{DISCLAIMER}

Portions of this document may be illegible in electronic image products. Images are produced from the best available original document. 
TT92 011579

\section{Evaluations of 1990 PRISM Design Revisions}

Manuscript Completed: January 1992

Date Published: March 1992

Prepared by

G. J. Van Tuyle, G. C. Slovik, B. C. Chan,

A. L. Aronson, R. J. Kennett

Brookhaven National Laboratory

Upton, NY 11973

Prepared for

Division of Regulatory Applications

Orfice of Nuclear Regulatory Research

U.S. Nuclear Regulatory Commission

Washington, DC 20555

NRC FIN A3827 
- 


\begin{abstract}
Analyses of the 1990 version of the PRISM Advanced Liquid Metal Reactor (ALMR) design are presented and discussed. Most of the calculations were performed using BNL computer codes, particularly SSC and MINET. In many cases, independent BNL calculations were compared against analyses presented by General Electric when they submitted the PRISM design revisions for evaluation by the Nuclear Regulatory Commission (NRC).

The current PRISM design utilizes the metallic fuel developed by Argonne National Laboratory (ANL) which facilitates the passive/ "inherent" shutdown mechanism that acts to shut down reactor power production whenever the system overheats. While most of the reactivity feedbacks are negative, and therefore tend to reduce power, the sodium density feedback is positive. Boiling of the sodium could add a significant amount of positive feedback and cause a power increase. Various postulated unscrammed events were examined by GE and/or BNL, and much of the analysis discussed in this report is focussed on this category of events.

For the most part, the BNL evaluations are consistent with the analyses submitted by General Electric for PRISM. The principal areas of concern are related to the performance of the EM pumps and the many facets related to the ternary metal fuel. The ternary metal fuel is still being developed, and many questions regarding the fuel may be resolved as ANL continues with its metal fuel development and testing program.
\end{abstract}



Abstract $\ldots \ldots \ldots \ldots \ldots \ldots \ldots \ldots \ldots \ldots \ldots \ldots \ldots \ldots \ldots \ldots \ldots \ldots \ldots \ldots \ldots$

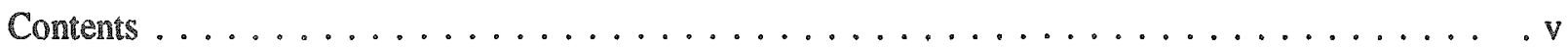

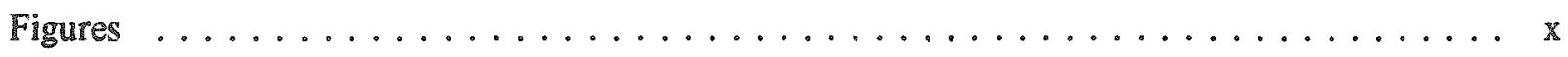

Tables $\ldots \ldots \ldots \ldots \ldots \ldots \ldots \ldots \ldots \ldots \ldots \ldots \ldots \ldots \ldots \ldots \ldots \ldots \ldots$

Executive Summary $\ldots \ldots \ldots \ldots \ldots \ldots \ldots \ldots \ldots \ldots \ldots \ldots \ldots \ldots$

Acknowledgements $\ldots \ldots \ldots \ldots \ldots \ldots \ldots \ldots \ldots \ldots \ldots \ldots \ldots \ldots \ldots \ldots$

1 Introduction $\ldots \ldots \ldots \ldots \ldots \ldots \ldots \ldots \ldots \ldots \ldots \ldots \ldots \ldots \ldots \ldots \ldots$

2 Comments Regarding Design Changes ..................... 2-1

2.1 Description of Design Changes and Pertinence $\ldots \ldots \ldots \ldots \ldots \ldots \ldots \ldots$

2.1.1 Increase in Reactor Power Level and System Power Production ........ 2-1

2.1 .2 Ultimate Shutdown System (USS) . . . . . . . . . . . . . . 2-1

2.1 .3 Gas Expansion Modules (GEMs) ................... 2 . 1

2.1.4 Mechanical Stops on Control Rods ................... 2-1

2.1.5 Below-Core Structure ....................... 2-2

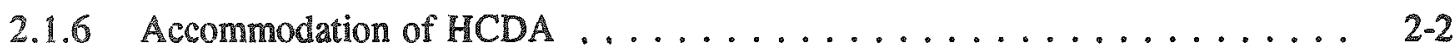

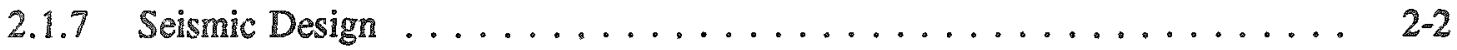

2.1.8 Containment Improvements and Dome . . . . . . . . . . . . 2-3

2.1 .9 Helical Coil Steam Generator . . . . . . . . . . . . . . . . 2-3

2.1.10 IHTS Auxiliary Cooling System Modifications . . . . . . . . . . . . . 2-3

2.1.11 Reactor Re-Design Outlined in Table G.2.2-2 . . . . . . . . . . . 2-3

2.2 Impact of Changes on Analyses $\ldots \ldots \ldots \ldots \ldots \ldots \ldots \ldots \ldots \ldots \ldots \ldots \ldots . \ldots \ldots$

2.2.1 Increase in Power Level ...................... 2-4

2.2 .2 Ultimate Shutdown System (USS) . . . . . . . . . . . . . . . 24

2.2 .3 Gas Expansion Modules (GEMs) ................... 2-4

2.2.4 Mechanical Stops on Control Rods .................... 2-5

2.2 .5 Below Core Structure ....................... 2-5

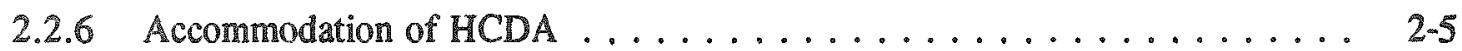

2.2 .7 Seismic Design .......................... $2-5$

2.2 .8 Containment Improvements . . . . . . . . . . . . . . . . $2-5$

2.2 .9 Helical Coil Steam Generator .................... $2-5$

2.2.10 IHTS Auxiliary Cooling System (ACS) Modifications . . . . . . . . . . $2-5$

2.2 .11 Reactor Re-Design ....................... 2-6 
3 Comments Regarding R \& D Programs In Support of Prism $\ldots \ldots \ldots \ldots \ldots \ldots$

3.1 R \& D Results Since $1986-87 \ldots \ldots \ldots \ldots \ldots \ldots \ldots \ldots \ldots \ldots$ 3-1

3.1.1 EM Pump Insulation $\ldots \ldots \ldots \ldots \ldots \ldots \ldots \ldots \ldots \ldots \ldots . \ldots \ldots$

3.1.2 Helical Coil Steam Generator $\ldots \ldots \ldots \ldots \ldots \ldots \ldots \ldots \ldots \ldots$ 3-1

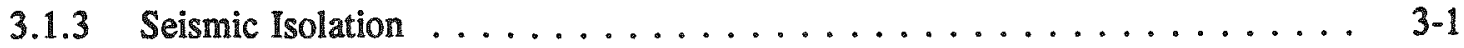

3.1.4 Thermal-Hydraulics Testing $\ldots \ldots \ldots \ldots \ldots \ldots \ldots \ldots . \ldots \ldots$ 3-1

3.1.5 Passive Reactivity Reduction $\ldots \ldots \ldots \ldots \ldots \ldots \ldots \ldots \ldots . \ldots \ldots$ 3-2

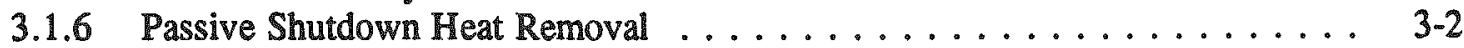

3.1.7 Safety and Licensing Support $\ldots \ldots \ldots \ldots \ldots \ldots \ldots \ldots \ldots . \ldots . \ldots .2$

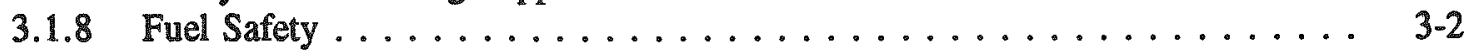

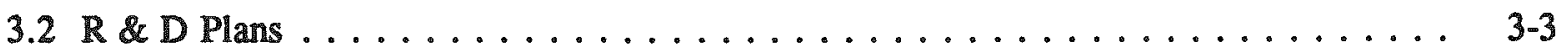

3.2.1 Plans in Support of ALMR (PRISM) $\ldots \ldots \ldots \ldots \ldots \ldots \ldots \ldots . \ldots \ldots$

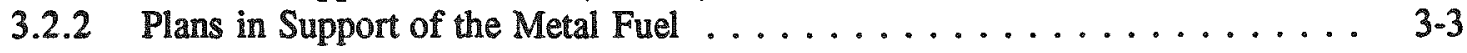

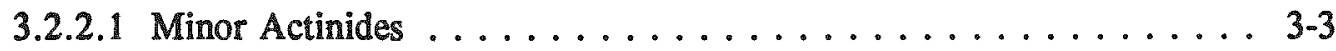

3.2.2.2 Fuel Cycle Facility: HFEF/South $\ldots \ldots \ldots \ldots \ldots \ldots \ldots .3-4$

3.2.2.3 Integral Fast Reactor (IFR) Technology Demonstration . . . . . . 3-4

3.3 Other Apparent R\&D Needs $\ldots \ldots \ldots \ldots \ldots \ldots \ldots \ldots \ldots \ldots . \ldots \ldots$

4 Analyses of Safety Issues Cited by GE in Appendix G.4 . . . . . . . . .

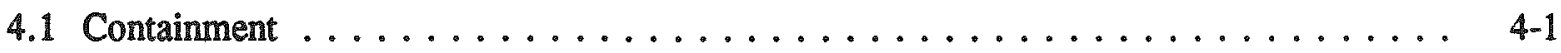

4.1.1 Revisions to the Containment Design ................ 4-1

4.1.2 Design Basis/Site Suitability Source Term $\ldots \ldots \ldots \ldots \ldots \ldots \ldots$. . . . . .

4.1.3 Containment Performance Analysis . . . . . . . . . . . 4 4

4.1.3.1 Applicant's Analysis of the Event ............ 4-4

4.1.3.2 BNL's Evaluation of the Applicant's Results $\ldots \ldots \ldots \ldots \ldots$. . . .

4.1.3.2.1 Total Energy Released by the Sodium Fire . . . . . . . 4-5

4.1.3.2.2 Rate of Combustion ... . . . . . . . . 4 4-5

4.1.3.2.3 Rate of Air Heat-Up . . . . . . . . . . 4 4-6

4.1.3.2.4 The Pressure Increase Over the First 4 Minutes. . . . . . 4-6

4.1.3.2.5 The Pressure Peak at 4 Minutes ........... 4-6

4.1.3.2.6 The Pressure Decrease after 4 Minutes ....... 4.7

4.1.3.2.7 Summary Assessment of CONTAIN Calculation . . . . . 4-7 
4.1.4 Oher Events Covered in Appendix G.4.1 ............... 4-8

4.2 Shutdown System $\ldots \ldots \ldots \ldots \ldots \ldots \ldots \ldots \ldots \ldots \ldots \ldots$

4.2.1 The Control Rod Stop System ..................... 4 .8

4.2.2 The Ulimate Shutdown System (USS) . . . . . . . . . . . . . 4-10

4.360 Year Plant Life $\ldots \ldots \ldots \ldots \ldots \ldots \ldots \ldots \ldots \ldots \ldots \ldots \ldots \ldots$

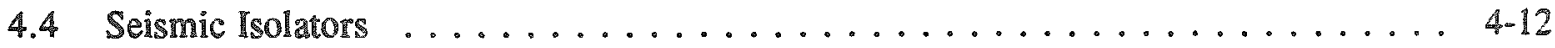

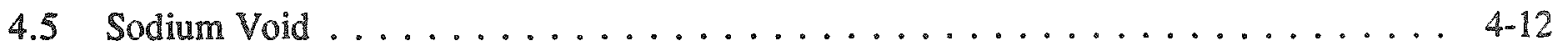

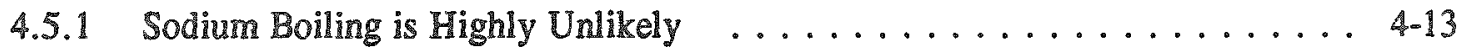

4.5.2 Can the HCDA Be Contained in the Vessel ? .............. 4-13

4.5.3 Can the Sodium Void Worth Be Reduced? ................. 4-14

4.5.3.1 Options Considered by ANL and GE ............ 4-14

4.5.3.2 Alternatives Not Considered by ANL and GE ............4-15

4.5.4 The Minor Actinide Factor ..................... . 4-19

4.6 Flow Blockage $\ldots \ldots \ldots \ldots \ldots \ldots \ldots \ldots \ldots \ldots \ldots \ldots \ldots \ldots \ldots . \ldots \ldots$

4.7 Electromagnetic Pumps $\ldots \ldots \ldots \ldots \ldots \ldots \ldots \ldots \ldots \ldots \ldots \ldots \ldots \ldots . \ldots \ldots$

4.7.1 EM Pump Characteristic ..................... 4-22

4.7 .2 Pump Control ............................ 4-. 4

4.7 .3 Synchronous Machines Isolated ................... 4-24

4.7 .4 Tuning the Coastdown ......................... 4.24

4.7 .5 Rods in Before Pump Trip ..................... 4-26

4.7.6 The 3 of 4 Pump Coastdown Case .................. 4.26

4.7 .7 EM Pumps at High Temperatures ................... 4.26

4.7 .8 The EM Pumps - Summary .................... 4.26

4.8 Sodium/Water Reaction Pressure Relief System (SWRPRS) . . . . . . . . . . 4-27

4.9 Reactor Vessel Auxiliary Cooling System (RVACS) . . . . . . . . . . . 4.28

4.9.1 Analytical Tools Used for RVACS Calculations ................ 4-29

4.9.2 PRISM System Conditions During RVACS Operation ............. . 4-29

4.9.3 Analyses of RVACS Performance During Reference LOHS .......... 4-29

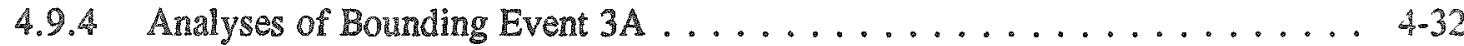

4.9 .5 Analyses of Bounding Event $3 B \ldots \ldots \ldots \ldots \ldots \ldots \ldots \ldots . \ldots \ldots . \ldots \ldots$

4.9 .6 Analyses of Vessel Leak Case .................... 4.32 
4.9.7 PRA Implications $\ldots \ldots \ldots \ldots \ldots \ldots \ldots \ldots \ldots \ldots \ldots \ldots \ldots$

4.10 Control Room $\ldots \ldots \ldots \ldots \ldots \ldots \ldots \ldots \ldots \ldots \ldots \ldots \ldots$

4.11 Emergency Preparedness $\ldots \ldots \ldots \ldots \ldots \ldots \ldots \ldots \ldots \ldots \ldots \ldots$

4.12 Role of Operator . . . . . . . . . . . . . . . . . . . . . . . . 4-39

4.13 Multi-Module Control . . . . . . . . . . . . . . . . . . . . . 4 4-39

4.14 Security $\ldots \ldots \ldots \ldots \ldots \ldots \ldots \ldots \ldots \ldots \ldots \ldots \ldots \ldots \ldots \ldots . \ldots \ldots$

4.15 Prototype Tests $\ldots \ldots \ldots \ldots \ldots \ldots \ldots \ldots \ldots \ldots \ldots \ldots \ldots$

4.16 Safety Analyses $\ldots \ldots \ldots \ldots \ldots \ldots \ldots \ldots \ldots \ldots \ldots \ldots \ldots \ldots$

4.16.1 SER Positions and Pertinence of Design Changes . . . . . . . . . . . . . 4-41

4.16.2 Summary of GE's Revised Results and BNL Interpretation . . . . . . . . . . 4-41

4.16.3 Revised Analyses of Core-Related Bounding Events (1 through 4) . . . . . . 4-41

4.16.3.1 Analytical Approach . . . . . . . . . . . . . . . 4-44

4.16.3.1.1 SCC Modeling [Ref. 21] . . . . . . . . . . . 4-44

4.16.3.1.2 MINET Representation [Refs. 19 and 20] . . . . . . . 4-48

4.16.3.2 Damage and Failure Limits ... . . . . . . . . . . . . . 4-48

4.16.3.3 Analyses of Individual Bounding Events $\ldots \ldots \ldots \ldots . \ldots .4 .53$

4.16.3.3.1 Unprotected Transient-Over-Power Events (UTOPs) . . 4-53

4.16.3.3.2 B.E. 2: Loss of Power . . . . . . . . . . . . . . . . 4.90

4.16.3.3.3 LOHS Events With Partial Blockage of RVACS Ducting 4-127

4.16.3.3.4 ULOF Missing One Pump Coastdown . . . . . . . 4-129

4.16.4 Gas Expansion Modules . . . . . . . . . . . . . . . . . . . 4-162

4.16 .5 Control Rod Stops ......................... 4-164

4.17 Station Blackout $\ldots \ldots \ldots \ldots \ldots \ldots \ldots \ldots \ldots \ldots$. . . . . . . . . . . . . . . . .

4.18 Risk Assessment $\ldots \ldots \ldots \ldots \ldots \ldots \ldots \ldots \ldots \ldots \ldots \ldots \ldots$

4.18 .1 Impact of Design Changes $\ldots \ldots \ldots \ldots \ldots \ldots \ldots \ldots \ldots \ldots \ldots$ 4-167

4.18.1.1 Seismic Isolation of the Synchronous Machines . . . . . . . . . 4-167

4.18.1.2 Strengthening Buildings and Enclosures . . . . . . . . . . . 4 4-167

4.18.1.3 Gas Expansion Modules (GEMs) . . . . . . . . . . . . . . . . 4-167

4.18.1.4 Ultimate Shutdown System (USS) . . . . . . . . . . . . . 4-167 
4.18.1.5 The Control Rod Stops ... . . . . . . . . . . . . . 4-168

4.18.1.6 Designing to Accommodate HCDAs ... . . . . . . . . . . 4-168

4.18.1.7 Designing to A ccommodate Core Meltdown . . . . . . . . . . 4-168

4.18.1.8 The Containment Dome ... . . . . . . . . . . . . 4-168

4.18 .2 Sensitivity Studies $\ldots \ldots \ldots \ldots \ldots \ldots \ldots \ldots \ldots$. . . . . . . . . . . . . .

4.19 Mitigation of Severe Core Accidents . . . . . . . . . . . . . . . . . . 4-169

4.19.1 Impact of HCDA on PRISM Vessel and Structures . . . . . . . . . . 4 4-170

4.19.2 Analysis of Molten Core on Support Plate ............. 4-170

5 Summary and Conclusions $\ldots \ldots \ldots \ldots \ldots \ldots \ldots \ldots \ldots \ldots \ldots \ldots \ldots$

6 References $\ldots \ldots \ldots \ldots \ldots \ldots \ldots \ldots \ldots \ldots \ldots \ldots \ldots \ldots \ldots \ldots \ldots$ 
1 PRISM Containment Dome and Vessel . . . . . . . . . . . . . . . . . 4-2

2 Control Rod Drive and $\operatorname{Rod}$ Stop Mechanism . . . . . . . . . . . . . . . . 4-9

3 Ultimate Shutdown Assembly $\ldots \ldots \ldots \ldots \ldots \ldots \ldots \ldots \ldots \ldots \ldots$

4a ANL and KfK Studies in Proceedings of 1990 Fast Reactor SafetyMeeting: by Cahalan, et al, and Royl, et al, respectively . . . . . . . . . . . . . . 4-16

4b Results from October 1990 Study of Omberg and Waltar for PRISM Core, Using Diffusion Codes and Neutron Transport Codes . . . . . . . . . . . . . . . . . . 4-16

5 Effect of Core Height on Sodium Void Reactivity Worth for Four LMR Fuel Forms in Soviet BN-1600 Type Reactor ........................ 4-17

6 Trade-Off Between Sodium Void Worth and Burnup Reactivity Swing for a Small ALMR Core. (Source: 10/90 WHC Study by A. Waltar and R. Omberg) . . . . . . . . . 4-18

7 Influence of Minor Actinide Content on Sodium Void and Doppler Reactivity Feedbacks . . . 4-20

8 PRISM EM Pump Head vs. Sodium Flow Rate $\ldots \ldots \ldots \ldots \ldots \ldots \ldots$

9 Predicted Flow Coastdown after a Trip from ARIES (G.E.) and MINET (BNL) . . . . . 4-25

10 PRISM During RVACS Cooling Event (No Vessel Leak) . . . . . . . . . . . . . 4-30

11 PRISM LOHS with RVACS Performing Normally . . . . . . . . . . . . . . 4-31

12 PRISM LOHS with RVACS Air Flow Ducting $75 \%$ Blocked . . . . . . . . . . . 4-33

13 PRISM LOHS with RVACS Blocked 12 Hours; Open $25 \%$ Thereafter . . . . . . . . . . . $4-34$

14 PRISM RVACS with Postulated Leak in Reactor Vessel . . . . . . . . . . . . . . 4 4-35

15 PRISM LOHS with Leak in Reactor Vessel (No Spill-Over) . . . . . . . . . . . . . . 4-36

16 Peak Fuel, Clad, and Sodium Temperatures for 4 Key Bounding Events and Pertinent Limits for Fuel, Cladding, Structures, and Sodium Boiling . . . . . . . . . . 4 4-43

17 SSC Representation of PRISM Reactor System . . . . . . . . . . . . . . . 4-45 
FIGURES (Cont'd)

Page

18 Operation of the Gas Expansion Module (GEM) Tested in FFTF (Which has a Similar Behavior in PRISM) $\ldots \ldots \ldots \ldots \ldots \ldots \ldots \ldots \ldots \ldots \ldots \ldots$

19 MINET Representation of PRISM System . . . . . . . . . . . . . . . . . 4 449

20 Relative Power Prediction from SSC for PRISM During a 40 Cent UTOP for Assumptions Similar to ARIES (i.e., GE Code) . . . . . . . . . . . . . . . . 4-56

21 Average Core Outlet Temperature Prediction from SSC for PRISM During a $40 c$ UTOP for Assumptions Similar to ARIES . . . . . . . . . . . . . . . . . . . 4-57

22 Predicted IHX Inlet and Outlet Temperature Predictions from SSC for PRISM During a 40 C UTOP for Assumptions Similar to ARIES . . . . . . . . . . . . . . . . 4-58

23 Reactivity Feedbacks Predicted for the 406 UTOP from SSC Using Conditions Similar to ARIES . . . . . . . . . . . . . . . . . . . . . . . . . . . 4 459

24 Reactivity Feedbacks Predicted for the 40C UTOP from SSC Using Conditions Similar to ARIES .................................. . 4.60

25 Reactivity Feedbacks Predicted for the 40C UTOP from SSC Using Conditions Similar to ARIES ................................... . 4 4 61

26 Predicted Fuel Temperature Distribution from SSC for the Third Node from the Top (i.e., $0.898 \mathrm{~m}-0.674 \mathrm{~m}$ ) Using Nominal Ternary Fuel Thermal Conductivities . . . . . . 4-62

27 Predicted Fuel Temperatures from SSC for the Second Node from the Top (i.e., $1.122 \mathrm{~m}-0.897 \mathrm{~m}$ ) for a 40 C UTOP Using Nominal Ternary Fuel Thermal Conductivities ................................. 4 4-63

28 Predicted Fuel Temperature Distribution for the Top $0.224 \mathrm{~m}$ (i.e., $1.346 \mathrm{~m}$ $1.122 \mathrm{~m}$ ) of the Fuel Pin Using Nominal Ternary Fuel Thermal Conductivities

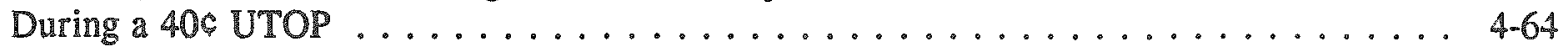

29 Relative Power Prediction from SSC for PRISM During a 409 UTOP . . . . . . . . . . 4-67

30 Average Core Outlet Temperature Predicted from SSC for PRISM During a 40c UTOP . . 4-68

31 Predicted Total, Above Core Load Pad (ACLP) and Core Support Grid Plate Reactivity Feedback from SSC for a 40 c UTOP . . . . . . . . . . . . . . . . . . . . . 4-69

32 Predicted Radial Expansion, Sodium Density (Density), and Axial Expansion Reactivity Feedback from SSC for a 40 C UTOP ....................... 4.70 
33 Predicted Doppler, Control Rod Expansion, and Gas Expansion Module (GEM) Reactivity Feedback from SSC for a $40 \mathrm{C}$ UTOP . . . . . . . . . . . . . . . . . . . . 4-71

34 Predicted Fuel Temperature Distribution from SSC for the Third Node From the Top (i.e., $0.897 \mathrm{~m}-0.673 \mathrm{~m}$ ) During a $40 \mathrm{C}$ UTOP . . . . . . . . . . . . . . . . . . . 4 4-72

35 Predicted Fuel Temperature Distribution from SSC for the Second Node from the Top (i.e., $1.122 \mathrm{~m}-0.897 \mathrm{~m}$ ) During a $40 \mathrm{c}$ UTOP . . . . . . . . . . . . . . . . . . 4 4-73

36 Predicted Fuel Temperature Distribution form SSC for the Top Node (i.e., $1.346 \mathrm{~m}-1.122 \mathrm{~m}$ ) . . . . . . . . . . . . . . . . . . . . . 4.74

37 Predicted Sodium Saturation Margin from SSC for the 40c UTOP . . . . . . . . . . . 4 4-75

38 Relative Power Prediction from SSC for PRISM During a 40 cent UTOP/LOF . . . . . . 4 4-79

39 Average Core Outlet Temperature Prediction from SSC During a 40c UTOP/LOF . . . . 4-80

40 Predicted Total, Above Core Load Pad (ACLP) and Core Support Grid Plate Reactivity Feedback from SSC for a $40 \mathrm{C}$ UTOP/LOF . . . . . . . . . . . . 4 4-81

41 Predicted Radial Expansion, Sodium Density (Density), and Axial Expansion Reactivity Feedback from SSC during a 40 C UTOP/LOF . . . . . . . . . . . . . 4-82

42 Predicted Doppler, Control Rod Expansion, and Gas Expansion Module (GEM) Reactivity Feedback from SSC during a $40 \mathrm{c}$ UTOP/LOF . . . . . . . . . . . . 4-83

43 Predicted Channel Exit Sodium Temperatures During a 40c UTOP/LOF . . . . . . . . . .4-84

44 Predicted Fuel Temperature Lisuribution from SSC for the Third Node from the Top (i.e., $0.897 \mathrm{~m}-0.673 \mathrm{~m}$ ) During a $40 \mathrm{C}$ UTOP/LOF . . . . . . . . . . 4 4-85

45 Predicted Fuel Temperature Distribution for the Top Node (i.e., $1.346 \mathrm{~m}$ -

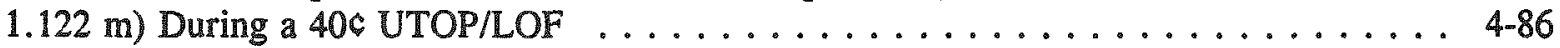

46 Predicted Sodium Saturation Margin from SSC for a 40c UTOP/LOF . . . . . . . . 4-87

47 Predicted Sodium Level in the GEMs from SSC for a $40 \mathrm{C}$ UTOP/LOF . . . . . . . . . 4-88

48 Peak Fuel, Clad, and Sodium Temperatures for UTOP Events: GE's 1A, BNL's 1A With and Without Pu Migration, and BNL's UTOP/LOF . . . . . . . . . . . 4-89 


\section{FIGURES (Cont'd)}

49 Predicted Relative Power and Core Flow from SSC During a ULOF/LOHS . . . . . . . . 4-91

50 Average Core Outlet Temperature Prediction from SSC During a ULOF/LOHS . . . . . 4 4-93

51 Predicted Total, Above Core Load Pad (ACLP) and Core Support Grid Plate Reactivity Feedback from SSC for a 40 C UTOP/LOHS . . . . . . . . . . . . . . 4-93

52 Predicted Radial Expansion, Sodium Density, and Axial Expansion Reactivity Feedback from SSC During a ULOF/LOHS ... . . . . . . . . . . . . . . 4 4-93

53 Predicted Doppler, Control Rod Expansion, and Gas Expansion Module (GEM) Reactivity Feedback from SSC during a 40 c ULOF/LOHS . . . . . . . . . . . . . . . 4-93

54 Predicted Sodium Level in the GEMs from SSC During a ULOF/LOHS . . . . . . . . 4 4-93

55 Predicted GEM Gas Pressure from SSC During a ULOF/LOHS . . . . . . . . . . . . 4-93

56 Predicted Fuel Temperature Distribution from SSC for the Top Node (i.e., $1.346 \mathrm{~m}-1.122 \mathrm{~m}$ ) During a ULOF/LOHS . . . . . . . . . . . . . . . $4-93$

57 Predicted Sodium Saturation Margin from SSC During a ULOF/LOHS . . . . . . . . 4-93

58 Predicted Relative Power from SSC for a ULOF with and without GEMs . . . . . . . . 4-93

59 Average Core Outlet Temperature from SSC for a ULOF With and Without GEMs . . . . 4 4-93

60 Predicted Fuel Temperature Distribution from SSC for the Third Node from the Top (i.e., $0.897 \mathrm{~m}-0.673 \mathrm{~m}$ ) for a ULOF with GEMs $\ldots \ldots \ldots \ldots \ldots \ldots \ldots$

61 Predicted Fuel Temperature Distribution from SSC for the Top Node (i.e., $1.346 \mathrm{~m}$ $1.122 \mathrm{~m}$ ) for a ULOF with GEMs $\ldots \ldots \ldots \ldots \ldots \ldots \ldots . \ldots \ldots$. . . . . . . . . . . . . . . . .

62 Predicted Total, ACLP, and GRID Plate Reactivity Feedback from SSC for a ULOF with GEMs . . . . . . . . . . . . . . . . . . . . . . . . 4 4-93

63 Predicted Radial Expansion, Sodium Density, and Axial Expansion Reactivity Feedback from SSC for a ULOF with GEMs

64 Predicted Doppler, Control Rod Expansion, and GEM Reactivity Feedback from SSC for a ULOF with GEMs . . . . . . . . . . . . . . . . . . . . . . 4 4-107

65 Predicted Margin to Sodium Saturation from SSC for a ULOF With and Without GEMs . . 4-109 
FIGURES (Cont'd)

Page

66 Predicted Total, ACLP, and GRID Plate Reactivity Feedback from SSC for a ULOF Without

GEMs .................................. . 4-110

67 Predicted Radial Expansion, Sodium Density, and Axial Expansion Reactivity Feedback from SSC for a ULOF Without GEMs $\ldots \ldots \ldots \ldots \ldots \ldots \ldots \ldots \ldots \ldots \ldots \ldots . \ldots \ldots$

68 Predicted Doppler, Control Rod Drive Line Expansion, and GEM Reactivity Feedback from SSC for a ULOF Without GEMs . . . . . . . . . . . . . . . . . . . . . . 4-112

69 Predicted Fuel Temperature Distribution from SSC for the Second Node from the Top (i.e., $1.122 \mathrm{~m}-0.897 \mathrm{~m}$ ) for a ULOF Without GEMs . . . . . . . . . . . . 4-113

70 Predicted Fuel Temperature Distribution from SSC for the Top Node (i.e., $1.346 \mathrm{~m}$ $1.122 \mathrm{~m}$ ) for a ULOF Without GEMs . . . . . . . . . . . . . . . . 4-114

71 Predicted Inlet and Outlet IHX Temperatures from SSC for a ULOHS . . . . . . . . . 4-116

72 Predicted Relative Power from SSC for a ULOHS $\ldots \ldots \ldots \ldots \ldots \ldots$. . . . . . . . . . 117

73 Predicted Core Inlet Plenum Bulk Average Temperature from SSC for a ULOHS . . . . 4-118

74 Predicted Average Core Outlet Sodium Temperature from SSC for a ULOHS $\ldots \ldots$. . . . 4-119

75 Predicted Total, ACLP, and GRID Plate Reactivity Feedback from SSC for a ULOHS . . 4 4-120

76 Predicted Radial Expansion, Sodium Density, and Axial Expansion Reactivity Feedback from $\mathrm{SSC}$ for a ULOHS . . . . . . . . . . . . . . . . . . . . . . . . . .

77 Predicted Doppler, Control Rod Expansion, and GEM Reactivity Feedback from SSC for a ULOHS.

78 Predicted Fuel Temperature Distribution from SSC for the Third Node from the Top (i.e., $0.897 \mathrm{~m}-0.673 \mathrm{~m}$ ) for a ULOHS

79 Predicted Fuel Temperature Distribution from SSC for the TOP Node (i.e., $1.346 \mathrm{~m}$ $1.122 \mathrm{~m}$ ) for a ULOHS . . . . . . . . . . . . . . . . . . . . . . 4-124

80 Predicted Margin to Sodium Saturation from SSC for a ULOHS . . . . . . . . . . . . 4-125

81 Peak Fuel, Clad, and Sodium Temperatures for Various Unscrammed Loss of Flow and/ or Loss of Heat Sink Events . . . . . . . . . . . . . . . . . . . . . . . . . 
82 Peak Reactor Outlet Sodium Temperatures for Scrammed LOHS (RVACS-Only) Events . $\begin{array}{r}\text { Page } \\ 4-128\end{array}$

83 Predicted Pump Flow Rate from MINET for a ULOF/LOHS with 4 EM Pumps . . . . . 4-130

84 Predicted Pump Flow from MINET for a ULOF/LOHS with a Pump Seizure and 3 Pumps

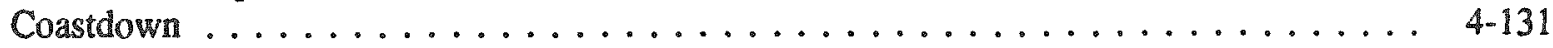

85 Predicted Flow in a Seized EM Pump for a ULOF/LOHS with Coastdown from 3 Pumps . $4-132$

86 Predicted Core Outlet Flow from MINET for a ULOF/LOHS with 1 Pump Seizure and 3 Pump Coastdown ............................. 4-133

87 Predicted Relative Power and Normalized Core Flow from SSC for a ULOF with 1 Pump Seized ............................... . . 4-134

88 Predicted Total, ACLP, and Grid Plate Reactivity Feedback from SSC or a ULOF with 1 Pump Seized . . . . . . . . . . . . . . . . . . . . . . . . 4-135

89 Predicted Core Radial Expansion, Sodium Density, and Axial Expansion Reactivity Feedback from SSC for a ULOF with 1 Pump Seized . . . . . . . . . . . . . . 4-136

90 Predicted Doppler, Control Rod Drive Line Thermal Expansion and GEM Reactivity Feedback from SSC for a ULOF with 1 Seized Pump . . . . . . . . . . . . . 4-137

91 Predicted Sodium Level in GEMs from SSC for a ULOF with 1 Pump Seized . . . . . . 4-139

92 Predicted Fuel Temperature Distribution for the Third from the Top Node (i.e., $0.897 \mathrm{~m}-0.673 \mathrm{~m}$ ) of a Fuel Pin from SSC for a ULOF with 1 Pump Seized . . . 4 4-140

93 Predicted Fuel Temperature Distribution for the Top Node (i.e., $1.346 \mathrm{~m}-1.122 \mathrm{~m}$ ) of a Fuel Pin from SSC for a ULOF with 1 Seized Pump . . . . . . . . . . . . . 4-141

94 Predicted Margin to Sodium Saturation from SSC for a ULOF with 1 Pump Seized . . . . 4-142

95 Core Exit Average Sodium Temperature from SSC for a ULOF with 1 Pump Seized . . . 4-143

96 Predicted Relative Power and Normalized Core Flow from SSC for a Pump Seizure . . . 4-144

97 Predicted Total, ACLP, and Core Support GRID Plate Reactivity Feedback from SSC for a Pump Seizure . . . . . . . . . . . . . . . . . . . . . . . 4-145

98 Predicted Core Radial Expansion, Sodium Density, and Axial Expansion Reactivity Feedback from SSC for a Pump Seizure . . . . . . . . . . . . . . . . . 4 4-146 


\section{FIGURES (Cont'd)}

99 Predicted Doppler, Control Rod Drive Thermal Expansion, and GEM Reactivity Feedback from $\mathrm{SSC}$ for a Pump Seizure . . . . . . . . . . . . . . . . . . . . 4-147

100 Predicted Fuel Temperature Distribution from SSC for the Third Node from the Top (i.e., $0.897 \mathrm{~m}-0.673 \mathrm{~m}$ ) for a Pump Seizure ................ . 4-148

101 Predicted Margin to Sodium Saturation from SSC for a Pump Seizure . . . . . . . . 4-149

102 Core Outlet Average Sodium Temperature from SSC for a Pump Seizure . . . . . . 4-150

103 Predicted IHX Inlet and Outlet Sodium Temperatures from SSC for a Pump Seizure . . 4-151

104 PRISM Core Sodium Flow Rates After 4 Pumps "Seize" . . . . . . . . . . . . . 4-153

105 Predicted Relative Power and Normalized Core Flow from SSC for a Pump Seizure . . . 4-154

106 Predicted Total, ACLP, and Core Support GRID Plate Reactivity Feedback from SSC for a Total EM Pump Failure $\ldots \ldots \ldots \ldots \ldots \ldots \ldots \ldots$. . . . . . . . . . . . . . . . . . .

107 Predicted Core Radial Expansion, Sodium Density, and Axial Expansion Reactivity Feedback from SSC for a Total EM Pump Failure . . . . . . . . . . . . . . . . 4-156

108 Predicted Doppler, Control Rod Drive Line Thermal Expansion, and GEM Reactivity Feedback from SSC for a Total EM Pump Failure . . . . . . . . . . . . . . . . 4-157

109 Predicted Sodium Level in the GEMs from SSC for a Total EM Pump Failure . . . . . 4-158

110 Predicted Fuel Temperature Distribution from SSC for the Third Node from the Top (i.e., $0.897 \mathrm{~m}-0.673 \mathrm{~m}$ ) for a Total EM Pump Failure . . . . . . . . . . . . . . . 4-159

111 Predicted Margin to Sodium Saturation from SSC for a Total EM Pump Failure . . . . 4-160

112 Channel Exit Sodium Temperature from SSC for a Total EM Pump Failure . . . . . . . 4-161

113 Peak Fuel, Clad, and Sodium Temperatures for Variations on the Unscrammed Loss of Flow Event . . . . . . . . . . . . . . . . . . . . . . . . . . . . 4-163 


\section{TABLES}

Page

1 Initial and Key Operating Parameters $\ldots \ldots \ldots \ldots \ldots \ldots \ldots \ldots \ldots \ldots$

2 Peak Temperatures Predicted by SSC and ARIES ................ 4.65

3 Referenced Thermal Conductivities ...................... 4-66

4 Peak Values for UTOP Variant Case . . . . . . . . . . . . . . . . . . . . 4-76 



\section{EXECUTIVE SUMMARY}

This report is part of a continuing process to evaluate the General Electric (GE) Advanced Liquid Metal Reactor (ALMR) concept known as PRISM. Analyses performed in order to evaluate the PRISM concept as documented in the original Preliminary Safety Information Document (PSID) are summarized in the draft Safety Evaluation Report (SER) [NUREG 1368] and detailed in a supporting BNL report [NUREG/CR-5364]. During early 1990, in response to comments included in the SER, GE chose to amend the PRISM PSID and added Amendments 12 and 13, which formed a new Appendix G. Because GE did not alter the first five volumes of the PRISM PSID (Appendix G became Volume 6), we have left the corresponding independent analyses in the earlier reports intact. The new independent analyses, corresponding to the revised PRISM design documented in the new Appendix G, is documented herein. In addition, material included in this report will be factored into the NRC staff's SER, which will take into account PSID Appendix G. For that reason, the outline of this report corresponds to that of GE's PSID Appendix G.

The recent design revisions, made largely in response to NRC concerns, have been quite significant and have changed some of our perceptions regarding the safety of this design. The increase in reactor power (for economic reasons) may have changed some safety margins, and the switch to a single-wall-tube helical coil steam generator may increase the likelihood of a tube leak and a sodiumwater reaction (note: this steam generator design also has good accident mitigation capabilities). Addition of the Ultimate Shutdown System (USS) and the containment dome are believed to be significant safety improvements. Modifications to the below-core structure, the seismic design (especially regarding the placements of the synchronous machines), and the IHTS Auxiliary Cooling System (ACS) are also considered to be substantial improvements. Some changes were also required to compensate for new test data for the ternary metal fuel. These changes include a reactor-redesign, and the addition of the GEMs and the control rod stop system.

Our overall evaluation of these changes is that the PRISM system design has been improved, but that a more detailed evaluation of the ternary metal fuel has revealed some apparent problems. The data collected to date clearly shows migration of the uranium and zirconium components during burnup, and suggests that some plutonium migration may also occur. In addition, the temperature cited by ANL for the onset of eutectic penetration at the fuel-cladding interface (970K) may not be conservative, as simple modeling and the early data suggest a lower limit (900K) may be more correct. It must be recognized that the ANL experience with the high Pu (26.5\%) ternary metal fuel has been quite limited, and that some problems should have been expected. More data is needed before ANL can resolve some of the current questions about plutonium migration and low eutectic temperatures.

The research and development program outlined by GE covers many of the higher priority needs, and includes some indications that there will be increased emphasis on examining factors important to accident mitigation. It is clear that most of the $R \& D$ support will be in the metal fuels area, particularly a fuel cycle facility (HFEF/South) and further testing in EBR-II. Part of this work will likely examine the impact of factoring minor actinides into the ternary fuel, which should take place if LWR spent fuel is to be used to produce the initial fuel loading for PRISM. If the only minor actinide feed for PRISM is that which comes along with the LWR spent fuel plutonium, the impact on core physics parameters may well be acceptable. (However, a special purpose machine having more actinides than uranium and plutonium would have some significant reactivity problems and would bear little resemblance to PRISM).

Although GE did not discuss their planned R\&D into key severe accident issues, such as rapid axial expulsion and the behavior of molten metal fuel in sodium pools, it is clear that they, along with $D O E$ and $A N L$, plan to increase the efforts in these areas. 
The bulk of GE's Appendix $G^{11}$ is addressed to 19 "Safety Issues", as interpreted by GE from the draft SER'.2. Some of our evaluations of these responses follow.

\section{Containment}

The PRISM reactor system is very different from light water reactors (LWRs), so one has quite different design objectives. With respect to a possible sodium fire event, analysis indicates the current containment design should properly contain a worst-case sodium pool fire. A principal concern regarding the PRISM containment is that data for metal fuel behavior during a severe accident is unavailable, so it is very difficult to characterize the potential challenges to the containment.

\section{The Shutdown System}

Because of the large positive sodium reactivity worth, and despite the passive reactor shutdown characteristic, the reactor shutdown system is one of the most important safety systems in PRISM. The main shutdown system, based on six independent control rod sub-systems, appears to be highly reliable and redundant. However, the recent addition of the Ultimate Shutdown System adds an alternate shutdown system and thereby largely addresses a previous concern. With respect to the Control Rod Stop System, it is needed to reduce TOP initiators to levels acceptable for the fuel in PRISM. However, some operational aspects of moving the small distances required for the control rod stops has not been completely explained by the vendor.

\section{Sodium Void}

GE and ANL are making progress in trying to address our concerns regarding the large positive sodium void reactivity worth. Clearly, no one wants this design characteristic, but the options for eliminating it are limited and have undesirable impacts on other design characteristics. GE's stated response has three components. First, the chances of the sodium bolling are very small (we concur). Second, if the sodium boils the resultant HCDA could probably be absorbed without catastrophic results (appears to be correct). Third, their most viable options for reducing the sodium void worth, using the metal fuel, is to "spoil" the geometry so as to increase neutron leakage and the "increased leakage" component of the sodium voiding reactivity feedback. Unfortunately, this increases the burnup reactivity swing, and therefore the potential size of the UTOP initiator.

\section{Sodium/Water Reaction Pressure Relief System (SWRPRS)}

By switching from the straight double-tube-wall steam generator (like EBR-II) to the helical-coil single-tube-wall steam generator, GE has probably increased the likelihood of a tube rupture but has also improved the capability to deal with such an event. The main objective of this system is to prevent damage to the IHX. The revised SWRPRS is likely to prevent damage to the IHX, based on both an active (isolation valves) and a passive (rupture disks plus carefully chosen piping elevations) sub-system that is included in the system.

"General Electric Advanced Nuclear Technology, "PRISM Preliminary Safety Information Document (PSID)," GEFR-00793. UC-87Ta, December 1987 (Limited Distribution).

(2) Landry, R. R., King, T. L., Wilson, J. N., "Draft Preapplication Safety Evaluated Report for Power Reactor Inherently Safe Module Liquid Metal Reactor," Nuclear Regulatory Commission Report, NUREG-1368, September 1989. 


\section{Reactor Vessel Auxiliary Cooling System (RVACS)}

RVACS is one of the most important safety systems in the PRISM design and seems very difficult to defeat. Our calculations have consistently confirmed those submitted by the applicant, and RVACS appears to be an excellent decay heat removal system. At this time, it is difficult to develop any reasonable failure probabilities for this system; however we suspect a massive seismic event may be the dominant contribution. There is a very subtle failure mode that may eventually prevail for this and other passive systems. This is based on system degradation coupled with a primary failure, e.g., the reactor vessel leaks and the heat transfer surfaces are fouled. Of course, this type of failure mode may be very low, but this could be the dominant mode for a system that is very highly failure resistant.

\section{Multi-Module Control}

A nine-module PRISM plant should be controlled by a state-of-the-art digital control system. The technology is available or can be readily developed. However, the size and complexity of such a system would make quality assurance and/or independent assessment a very formidable task. The more practical approach would be to determine that the reactor is sufficiently well protected by the Reactor Protection System (RPS) and by the passive response characteristics, such that errors generated by the control system would not be damaging.

\section{Prototype Tests}

We consider the prototype testing of the first PRISM module to be quite desirable, particularly with respect to the passive shutdown response and fuel performance. The vendor has expressed a desire to substitute an air-dump heat exchanger for the steam generator and sodium-water reaction protection relief system. The prototype would be much cheaper without these large and complex systems, and the impact on the test program would be minimal regarding determining core performance. However, some testing of these systems would be necessary, perhaps using some separate facilities, and they could be added later, if needed.

\section{Safety Analyses}

Several postulated events were analyzed using independent codes, such as SSC and MINET. In many cases, we could duplicate GE's calculations. However, the BNL calculations were made conservative.

Generally speaking, the PRISM RVACS has consistently stood up to close scrutiny, and it appears to be a very robust system. It is difficult to adequately factor this system into a PRA, because most failure modes are barely credible. We suspect the most plausible failure mode may be a single failure (blockage of one duct or a leak in the reactor vessel, for example) in combination with previously unnoticed degradation in some key performance parameters. While this combination may be unlikely, it may be more likely than either a double vessel failure or the complete blockage of all four air ducts.

The situation regarding the passive reactor shutdown is far more complex, especially since the flow of data for ternary metal fuel has only recently begun. Most of the metal fuel characteristics are based on extrapolations from binary data or a few ternary experiments which have either different cladding or PU concentrations. More data must be collected since the ternary metal fuel behavior appears to be very complex and there is evidence of some undesirable redistribution of the uranium, zirconium, and perhaps the plutonium components. In addition, the potential for eutectic penetration at the fuelcladding interface at relatively low temperatures appears to be possible. Some changes may be needed 
if the new data does not support the higher eutectic temperatures now postulated by ANL.

The greatest challenge, at the moment, appears to be the unprotected transient-over-power (UTOP) event, which boosts fuel centerline temperatures. The peak temperatures of a $40 \mathrm{c}$ initiator may be too large, given our current knowledge of the ternary fuel and the associated solidus temperature. GE may need to adjust the rod stops more frequently so as to reduce the largest TOP initiator into the $30 \mathrm{c}$ range to reduce peak center line temperatures. The vendor clearly has some options in this area, so it seems wise to withhold judgement here until GE proposes a final design.

Because of the GEMs, the power drops quickly with flow, and this has increased the safety margins for the ULOF events when compared to the previous design. As long as there is at least a partial coastdown provided by the synchronous machines, analysis indicates that PRISM would survive the ULOF category events.

The PRISM passive shutdown has always appeared to perform well for the ULOHS events, and nothing has changed significantly with the revised design. If there are problems here, they will develop only if the event continues for a long time, and the addition of the USS makes this very unlikely.

If there is a concern with normal operating events it will be at the fuel-cladding interface. Early data extrapolated from binary fuel or ternary fuel with the wrong Pu concentration or cladding indicates that the rate of eutectic formation at the temperatures experienced during the unscrammed events is very slow, so minimal damage is to be expected. However, the initial data and some simple analysis (thermal analysis only) suggest the eutectic formation may begin about $70 \mathrm{~K}$ lower than design criteria has been assuming. If the new data confirms the lower temperature limits, GE may need to make some materials substitutions or other design changes to account for the fuel-clad chemical interaction.

In summary, many of the PRISM reactor system changes must be considered improvements from the previous design. The increase in the reactor power production may have reduced some of the safety margins, although in most cases the margins are still substantial. Ironically, the principal safety issue remaining is the performance of the ternary metal fuel. Certainly there are some clear advantages to using the metal fuel, and the fuel developers seem very confident that any remaining technical problems can be resolved. Until more work has been completed on the ternary metal fuel protypical of PRISM, one can only say that the metal fuel has the potential to be an excellent fuel in a liquid metal cooled reactor. 


\section{ACKNOWLEDGEMENTS}

The Nuclear Regulatory Commission's (NRC's) effort to evaluate the various proposed advanced reactor concepts has already gone through a couple of iterations, so several staff members have been involved in the review of the advanced liquid metal reactors. The staffers who have been directly involved in evaluating the revisions to the PRISM design include Dr. Ralph Landry, Mr. Jerry Wilson, and Mr. Ed Throm. All actively and enthusiastically contributed to the effort to explore and understand this very novel reactor system design, and provided very useful insights and feedback regarding this report. We are indebted to them for their expert advice, as well as their support of our program. More recently, Mr. Robert Pierson, Mr. Edward Goodwin, Mr. John C. Lane, and Mr. Stephen Sands have become actively involved in pre-application reviews of PRISM, and provided very helpful suggestions regarding this report.

The "applicants" in the case of PRISM are General Electric, their sponsors at the Department of Energy, and their technical supporters at the Argonne National Laboratory. To some degree, representatives of these organizations were obligated to interact with NRC and its contractors. However, several of the individuals were especially helpful in responding to our questions and requests for further information. Mr. Nick Grossman (DOE), Dr. Geza Gyorey (GE), Dr. Par Magee (GE), Mr. Richard Hardy (GE), and Dr. Dean Pederson (ANL) were immensely helpful.

Finally, this report is both lengthy and complex. The responsibility of editing it fell upon the shoulders of Dr. James Guppy and Dr. Robert Bari, and their efiorts are much appreciated. Finally the process of typing, assembling, and cutting-and-pasting the numerous figures was ably managed by $M$ s. Kathy Ratto, with assistance from Mrs. Linda Hanlon. 


\section{INTRODUCTION}

This report is part of a continuing process to evaluate the General Electric (GE) Advanced Liquid Metal Reactor (ALMR) concept known as PRISM [Ref. 1]. Analyses performed in order to evaluate the PRISM concept as documented in the original Preliminary Safety Information Document (PSID) are summarized in the draft Safety Evaluation Report (SER) [Ref. 2] and detailed in a supporting BNL report [Ref. 3]. During early 1990, in response to comments included in the SER, GE chose to amend the PRISM PSID and added Amendments 12 and 13, which formed a new Appendix $G^{3}$. Because GE did not alter the first five volumes of the PRISM PSID (Appendix G became Volume 6), we have left the corresponding independent analyses in the earlier reports intact. The new independent analyses, corresponding to the revised PRISM design documented in the new Appendix $G$, is documented herein. In addition, material included in this report will be factored into the NRC staff's SER, which will take into account PSID Appendix G. For that reason, the outline of this report corresponds to that of GE's PSID Appendix G.

In responding to the SER, GE switched to a newer system design (already under evaluation by DOE) and responded to concerns expressed by the NRC staff, some of which originated as part of the independent evaluations performed by $\mathrm{BNL}$. In reviewing Appendix $\mathrm{G}$, we have deferred to the NRC in several instances where GE is responding to a concern expressed originally by NRC staff (i.e., not a concern originally flagged by $\mathrm{BNL}$ ) or where GE's response to a concern is based on a licensing position as opposed to a design change or new analyses.

Appendix $\mathrm{G}$ was written by GE primarily to help resolve NRC concerns through design revisions, improved analysis, or increased R\&D commitments. However, because of the higher power level of the newer design and some new information on performance of the ternary metal fuel alloys, there are new concerns regarding the safety of PRISM. Thus, certain design improvements, such as the new containment dome and a new "ultimate shutdown" device, are partially offset.

The design changes cited by GE and the impact these changes have on prior analyses and conclusions are discussed in Section 2. The R\&D program cited in Appendix $G$ is discussed in Section 3, which includes comments regarding where further R\&D needs should be considered by DOE. Several of the 19 Safety Issues cited by GE and discussed in the 19 sections of Appendix G.4 are covered in Section 4 of this report (some are deferred to the NRC staff for evaluation). Our findings regarding the revised PRISM design and analyses are summarized in Section 5.

\footnotetext{
(3) General Electric Advanced Nuclear Technology, "PRISM Preliminary Safety Information Document (PSID)." GEFR-00793, UC-87Ta, December 1987 (Limited Distribution).
} 


\section{COMMENTS REGARDING DESIGN CHANGES}

This section is divided into two sub-sections. In Secrion 2.1 the design change is briefly described and its pertinence to sarety is discussed. In Section 2.2 the impact on the analysis of key postulated evenis is discussed.

\subsection{Description of Design Changes and Pertinence}

\subsubsection{Increase in Reactor Power Level and System Power Production}

The reactor power level and the nine-reactor-system power production were increased from 425 MWt and $1245 \mathrm{MWe}$ to $471 \mathrm{MWt}$ and $1395 \mathrm{MWe}$, respectively. This was done primarily for economic reasons at the direction of DOE. GE did not re-size the key decay heat removal system, RVACS, and this results in higher temperatures during postulated accident conditions. In addition, the normal system operating temperatures are $17 \mathrm{~K}(30 \mathrm{~F})$ higher, so all event analyses had to be revised, accounting for hotter initial conditions.

\subsubsection{Ultimate Shutdown System (USS)}

While the passive reactor shutdown mechanism, based on reactivity feedbacks, has significant safety advantages, it usually leaves the reactor in a critical condition and therefore exposed to further changes in system conditions. With the addition of the USS, GE has provided an alternate means of shutting down the reactor. In this case, many small spheres of $\mathrm{B}_{4} \mathrm{C}$ are released to fall through a tube into the center of the core, in response to an operator actuated shutdown command. The device fills an important gap in the PRISM safety defenses. That is, the passive shutdown no longer has to function indefinitely, as a neutronic shutdown can be anticipated within some reasonable time frame.

\subsubsection{Gas Expansion Modules (GEMs)}

A key question regarding the passive shutdown mechanism is whether it can act to reduce reactor power quickly enough to prevent sodium boiling or fuel damage. The crucial test is the Unprotected Loss of Flow (ULOF) Case, which results in a relatively quick reduction in coolant flow to the reactor. Initially, the applicant believed that the reactivity feedbacks and their associated uncertainties were such that the passive shutdown could function effectively without GEMs in response to the postulated ULOF.

GEMs are simple devices, resembling large inverted test-tubes, containing a trapped region of gas above the core during normal operating conditions. They are placed on the perimeter of a reactor, and increase the neutron leakage and thereby reduce reactivity whenever the gas drops into the active core region. Under full pumping conditions, the gas pressure is high enough so sodium occupies that portion of the GEM which resides in the active core and traps the gas above the core. When the pumps stop and the system pressure falls, the gas region expands into the core, speeding the decrease in reactor power through increased leakage of neutrons.

\subsubsection{Mechanical Stops on Control Rods}

A key safety question regarding the passive shutlown, particularly with the use of metal fuel and its small Doppler reactivity feedback, is how much reactivity can be added to the core by withdrawing the control rods and whether the resulting power increase can be safely accommodated. In the original PRISM reactor design, GE and the metal fuel experts at ANL felt they could predict the burn-up 
reactivity swing to be less than $35 \mathrm{c}$, including a degree of uncertainty. More recent fuel data and fuel cycle analysis indicates that the burnup reactivity swing, due in part to significant axial expansion in the fuel, could be larger than was first believed. As the passive accommodation of reactivity additions is limited to roughly $40 \mathrm{c}$, some means was needed to limit the amount that could be inserted during the period when the control rod insertion was much higher, e.g., one dollar. This was done by placing electronically-controlled mechanical rod stops on the control rods. These would be positioned to limit the amount the rod could be removed from the core, and adjusted a few times during burn-up to assure that the possible reactivity insertion would be limited to a manageable level. Assuming the control rods are at mid-core (limiting), the maximum feedback for the movement of all six rods would be 75 cents/inch. Thus, a relatively large measurement error of $1 / 8$ inch would introduce a less than 10 cents error. However, there may be larger uncertainties involved in predicting control rod worth at the new position, so it remains to be determined whether the 10 cent uncertainty margin is sufficient.

\subsubsection{Below-Core Structure}

The original PRISM design relied almost exclusively on accident prevention and placed little emphasis on mitigation. More recently, however, GE and ANL have been addressing accident mitigation concerns, and the revision to the below-core structure is intended to result in better accommodation of a core-melt event. However, the data base regarding metal fuel failure under melt conditions or during postulated power excursions is incomplete, so it is difficult to evaluate the performance of such a structure under severe accident conditions. The analysis suggests that such a below-core structure would accommodate a melt and would probably result in a less-than-critical-mass. However, it is currently very difficult to predict behavior of the metal fuel during the transition period, as it relocates from the core configuration to the below-core structure. It is not clear how soon such data will become available, but it may take a few years to develop such a data base.

\subsubsection{Accommodation of HCDA}

The key factor that would terminate a power excursion would be the rapid axial extrusion of the fuel, which is expected to push rapidly ("pop") up into the fission gas plenum, effectively expanding the core and increasing neutron leakage, i.e., shutting down the reactor. To be effective, this expansion needs to take place very quickly. The fastest data that ANL has for metal fuel axial expansion is for a time period on the order of about eight seconds (the TREAT facility). In contrast, data from the international in-pile CABRI [Ref.4] test program (co-sponsored by US NRC through early 1985) describes oxide fuel axial expansion on a millisecond scale.

When one considers the applicant's analysis and some cross-comparisons of key parameters, it appears that the PRISM structures could accommodate a fairly large HCDA event. Thus, it is entirely possible that the applicant will establish that the PRISM vessels, head, and containment designs can withstand some design basis HCDA without failing catastrophically.

\subsubsection{Seismic Design}

GE has moved two key systems, i.e., the EM Pump Synchronous Machines and the Reactor Protection System (RPS) Electronics, into the seismically isolated region. Seismic isolation provides some protection against horizontal ground acceleration, and the isolation of these two key safety systems seems to be a significant improvement. In particular, BNL had previous concerns regarding cables running between the non-isolated synchronous machines and the isolated EM pumps. 


\subsubsection{Containment Improvements and Dome}

GE has improved the PRISM containment design, making more of the system leak-tight and adding a containment dome over the reactor head. In addition, they added isolation valves in the IHTS. This was done as part of an effort to strengthen the design regarding accident mitigation. The second vessel, called either a guard vessel or a containment vessel, is cooled by outside air as part of the RVACS heat removal system. This has clear advantages for preventing serious accidents in PRISM. The behavior of fission products and actinides from the metal fuel in either molten metal fuel or a sodium pool has never been well characterized. Therefore, it isn't known how many and how much of the most hazardous components would escape from the fuel and the sodium pool and make their way into the containment dome. Further, if it is not clear whether the containment design basis should be a core melt, a sodium fire, or an energetic event (see also Section 4.1)

\subsubsection{Helical Coil Steam Generator}

The applicant has substituted a single wall tube (thickness) helical coil steam generator in place of an older double wall tube concept which was based on the steam generators used in EBR-II. The older design was very conservative, and leakages between the sodium and water/steam were quite unlikely. The newer design may be more likely to suffer such a leak, but has the additional capability to accommodate any resulting sodium expansion and a capability to bypass the sodium-water reaction byproducts (see Section 4.8).

\subsubsection{IHTS Auxiliary Cooling System Modifications}

The applicant has added a forced circulation capability to this system, which removes heat from the outside of the steam generator. This is an "investment protection" system, and the forced circulation capability will likely reduce the time required to cool down the reactor in the event of a loss of normal cooling. As this is not a safety grade system, it was not evaluated in detail by BNL. However, the addition of a forced circulation capability would seem to be helpful to safety, since the system can still function under natural circulation.

\subsubsection{Reactor Re-Design Outlined in Table G.2.2-2}

As well as the addition of 3 GEMs and 1 USS to the core, the applicant has made various changes in pin size, fuel loading, power densities, and burnup. Most of these changes were made in response to some new information on the ternary metallic fuel, as well as a more thorough examination of reactor performance at different times in the fuel cycle. With these devices, the response of the reactor to unscrammed events may be a little betier on balance, although there are some problem areas not previously perceived. The performance of the revised reactor design is discussed throughout much of this report.

\section{Key Issues/Concerns in Section 2.1}

- While the addition of GEMs improves the passive shutdown response for ULOF events, are there instances where the GEMs could add reactivity or fail to function when needed? What would be the outcome of such failures and could these be prevented?

- Once one introduces control rod stops to limit potential UTOP initiators, one must determine a limit, e.g., 30c, and define the accuracy of the Rod Stop positioning. If the accuracy of the Rod Stop positioning is poor in comparison to the acceptable UTOP 
limit, then there would be a safety issue regarding the Rod Stops.

- Regarding accommodation of HCDAs, there is not sufficient data to confidently predict the size of an HCDA in a metal fuel ALMR. Therefore, the likelihood of an HCDA being accommodated within the PRISM vessel may remain an open issue until more data becomes available.

\subsection{Impact of Changes on Analyses}

\subsubsection{Increase in Power Level}

The increase in power level and other adjustments to the estimated decay heat curves resulted in a significant (15 to $20 \%$ ) increase in the decay heat that must be removed through RVACS in the case of a loss of heat sink event. (Note: the largest impact of minor actinides would be for the first cores, from LWR spent fuel. However, the largest decay heat will be in equilibrium cores, at end-of-life, and the minor actinide impact should be relatively small at that time). Previously the safety margins were quite high, and even simple models could demonstrate the effectiveness of RVACS. With the higher decay heat loads, one must be more precise in analyzing the RVACS performance. Fortunately, this was possible using slight modifications of existing analytical tools, and the agreement of the revised analyses with the applicant's calculational results is very close. While the safety margins are reduced, the performance of RVACS is still fairly impressive, and this is not viewed as a major problem area.

\subsubsection{Ultimate Shutdown System (USS)}

BNL's prior analyses of the postulated unscrammed events were terminated after a few minutes because (1) the worst of the transient was believed to be over and (2) there were shortcomings in our physical models in SSC when utilized beyond the first several minutes. With the introduction of the USS, it seems unlikely that an unscrammed transient would be allowed to continue indefinitely. Thus, the analysis of such postulated events beyond five or six minutes may no longer be necessary, depending on how long one assumes it takes for the operators to actuate the USS (it takes about one minute for shutdown after it is activated).

\subsubsection{Gas Expansion Modules (GEMs)}

The GEMs are important contributors to the mitigation of postulated unscrammed events involving loss of pumping, and they must be modeled explicitly. Therefore, models were added to the SSC representation of the PRISM reactor, and factored into the analyses of all postulated unscrammed events. It is noted that the potential impact of the GEMs is so significant that their usage in FFTF was sufficient to ensure that an oxide-fuel core could survive an unscrammed loss-of-flow. The impact on the PRISM ULOF analysis is very substantial.

There are some potential drawbacks associated with the GEMs, including the obvious questions about their failure to operate when needed, which could happen if some of the gas leaked out during normal operation, filling the GEM with sodium. Alternately, if the pumps are turned on while the reactor is critical, a significant power increase would result. Further, addition of the GEMs raises questions about shielding (more neutrons escape through the gas) and possible reactivity insertions should the gas escape and migrate into the central regions of the core. 


\subsubsection{Mechanical Stops on Control Rods}

The applicant argues that by adjusting the rod stops at proper intervals they can keep the potential TOP initiator below $40 \mathrm{c}$ ( $30 \mathrm{c}$ plus $10 \mathrm{c}$ of margin for error). While we must reserve judgement as to whether $40 \mathrm{c}$ is indeed a credible upper bound, we used 400 as the UTOP initiator for our revised analyses.

\subsubsection{Below Core Structure}

No attempt was made to perform detailed analysis of core melt or core disruptive events because the data base for molten metal fuel is incomplete. Arguments by the applicant regarding the geometry of the melt when imposed on the below core structure seemed consistent, with a major consideration being the porosity of the fuel- which is a key factor in cooling the melt.

\subsubsection{Accommodation of HCDA}

There is no data base to indicate how quickly the metal fuel will undergo extrusion, which is a rapid form of axial expansion and the key factor in limiting the size of the HCDA. Arguments made by the applicant regarding extrapolating from oxide fuel sound reasonable, but more data is required before one can make a final judgement regarding accommodation of an HCDA in PRISM.

\subsubsection{Seismic Design}

The impact of changes in the seismic design would be clear in any revision of the PRISM PRA. However, the applicant did not perform such a revision and BNL did not attempt to project what such a revision would look like. There was no impact from the changes, e.g., movement of the synchronous machines into seismic isolation, on the deterministic analyses of postulated events.

\subsubsection{Containment Improvements}

The applicant provided an analysis of a postulated sodium fire, which included the simulation of conditions in the containment as well as a source term calculation. Simple calculations confirmed the trends in conditions within the containment, and also revealed a small error in GE's analysis using the CONTAIN Code (see Section 4.1.3.2.6). However, the source term calculation depends strongly on assumptions regarding how many and how much of the key isotopes escape from the fuel, the sodium, the cover gas, and into the containment. The impact of the containment dome is believed to be fairly small in comparison to these other factors, with respect to limiting the source term.

\subsubsection{Helical Coil Steam Generator}

Because of the projected effectiveness of the RVACS in removing after-heat, our focus has not been on the steam generator. By this design change, the applicant may have increased the likelihood of a sodium-water reaction, which would be reflected in a revision to the PRA. However, a design feature of the new unit should help accommodate such an accident (see Section 4.8). Neither the old stearn generator nor the new design was explicitly factored into our calculations.

\subsubsection{IHTS Auxiliary Cooling System (ACS) Modifications}

Because the ACS is not a safety grade system, it has not been factored into BNL deterministic analyses. If it was to be factored in, it would likely be the natural circulation mode of operation that 
would be considered, as opposed to the new forced cooling operational mode.

\subsubsection{Reactor Re-Design}

The revisions to the PRISM reactor design were fairly extensive and all analyses for unscrammed events had to be repeated. Due to the addition of the GEMs and additional information regarding performance of the ternary metal fuel, our principal area of concern has shifted from the unscrammed loss of flow events to postulated transient-over-power events.

\section{Key Issues/Concerns in Section 2.2}

- Introduction of the GEMs could impact on various types of analysis. Their contribution during the basic unscrammed events (ULOF, ULOHS, UTOP) is significant and generally helpful. However, the GEMs will become an integral part of the core, and will have to be factored into many types of analysis, including reliability-based analysis.

- With respect to the analyses of severe accidents, some bounding analysis can be helpful until better data becomes available. However, there remains a great deal of work to be completed in this area, and this will continue to be true for at least two or three years.

- New data on the ternary metal fuel indicates there is much more to be learned, especially with respect to phase transitions. The ternary fuel is clearly different from the U-Zr fuel, and the data base is being built at this time. This is a key issue, especially during overpower events. 


\section{COMMENTS REGARDING RESEARCH AND DEVELOPMENT PROGRAMS IN SUPPORT OF PRISM}

The applicant used Appendix $G$ to discuss recent results from the supporting $R \& D$ program and to indicate planned Research and Development efforts, which include some increased efforts in the area of accident mitigation. The subsections below contain commentary regarding recent safety-related $R \& D$ accomplishments, planned R\&D, and some BNL concerns regarding the planned R\&D.

\subsection{Research and Development Results Since 1986-87}

\subsubsection{EM Pump Insulation}

Various potential electrical insulation materials are being testing for prolonged (accelerated) aging periods at high temperatures. This insulation is important to safety, as the loss of current to an EM pump. will result in an nearly instantaneous stoppage in pumping. Such a stoppage at full power would be very undesirable, although a reactor scram or a "passive shutdown" would prevent any serious damage to the reactor. However, should more than one pump stop this way, or should such a failure occur under adverse (very hot) conditions, the safety of the reactor could be very much dependent on achieving a successful reactor scram. It is still too early to explicitly factor the EM pump insulating materials into accident analyses, although this could be a very important common mode failure mechanism.

\subsubsection{Helical Coil Steam Generator}

Testing of a $70 \mathrm{MW}$ unit at ETEC was discontinued in 1989 after some 667 days of testing. This testing included operation at various conditions and included several transients. While these test results are likely to support the applicant's effort to implement a similar unit in PRISM, and to perform transient calculations and control system studies, our greater interest would be the resistance of the unit to tube leaks and the accommodation of any sodium-water reactions so as to prevent any damage to the $\mathbb{H X}$. If there was any testing performed in this area (beyond the testing of normal operational modes for nearly 2 years), it was not mentioned in Appendix G.

\subsubsection{Seismic Isolation}

A data base in support of the use of seismic isolators in PRISM is under development. This program involves ETEC and UC, Berkeley, and includes both laboratory scale tests and utilization in supporting new buildings. At one building in San Bernardino, a $.15 \mathrm{~g}$ acceleration at the basemat was experienced during a February 1990 earthquake. The building response was reported to be close to prior predictions.

\subsubsection{Thermal-Hydraulics Testing}

ANL has a 1/5 scale model of the PRISM vessel, with clear plexiglass used to represent the structures within the vessel and water used to represent the sodium coolant. Laser techniques are used to evaluate the flow fields under normal and off-normal conditions, and the results are used for both flow visualization and for comparison with 3-D code (COMMIX) calculations. At this time, it does not appear that 3-D flow patterns are crucial to the safety of PRISM, due to the relatively small pools and the high thermal conductivity of the sodium, but there may be cases where it could become important. Therefore, the facility may be of more interest in future evaluations of PRISM. 


\subsubsection{Passive Reactivity Reduction}

The ANL unscrammed test series at the EBR-II reactor in Idaho continue to be very successful. It is quite clear that the small metal fuel reactor has a very strong tendency to effectively shut down whenever it is overheated. This is clear not only from the test results, but also from the best-estimates of the reactivity feedbacks, which are all significantly negative. In particular, the negative sodium temperature reactivity feedback is an added layer of protection, because increases in the coolant temperatures act to bring the power level down. However, there are at least two problems on extrapolating from EBR-II to PRISM. First, the positive sodium temperature reactivity feedback in the larger PRISM core is a major safety concern, and is very different from that in EBR-II. Second, EBR-II uses primarily uranium-based fuel, although elements containing some plutonium are gradually being substituted into the core. Since PRISM is to use $26 \% \mathrm{Pu}$ in the metal fuel, the extrapolation from the recent tests at EBR-II is non-trivial.

The Westinghouse Hanford Corporation tests at FFTF have also recorded some successes, although aided in part by incorporation of GEMs in the core. GEMs are needed with oxide fuel, as the large Doppler reactivity feedback tends to hold up any power decrease and prevents the passive shutdown from being effective enough for some faster transients.

\subsubsection{Passive Shutdown Heat Removal}

Several tests were performed at the ANL facility representing the air-side RVACS heat removal. This large facility represents an annular sector of RVACS in full length. Testing has largely confirmed ANL models of the system, and results have been used to benchmark some of ANL's computer models for simulating RVACS performance under various conditions.

\subsubsection{Safety and Licensing Support}

This appears as an item in Table G.3.1-1 [Ref. 1] with the comment "Supplemental data base and models for characterization of radionuclide transport and residual risk as necessary." This is undoubtedly in reference to the GE/DOE/ANL intention to expand the metal fuels data base as necessary with respect to severe accident mitigation. It appears that this program is still in the planning stages.

\subsubsection{Fuel Safety}

ANL is continuing to run further tests at TREAT, EBR-II, and some out-of-pile materials test facilities. One major issue here is the $26 \% \mathrm{Pu}$ fuel that is to be used in PRISM, as ANL has only recently begun to collect data at low burnup for this fuel . It is noted that the ternary fuel behavior is somewhat different than that of the uranium metal fuel, so ANL may need a fairly substantial test program in support of the high Pu PRISM fuel. In particular, ANL must better characterize fuel component migration and low temperature eutectic formulation at the fuel-cladding interface. A second major need is for fuel extrusion (rapid axial expansion) data in response to fast transients (see also Section 2.1.6 of this report), and this may be very difficult data to develop. Therefore, there is a very substantial need for more data on the PRISM fuel, and a large ongoing ternary fuel test program at ANL will likely be needed to support PRISM in future evaluations.

Key Issues/Concerns in Section 3.1

- Past metal fuel efforts have de-emphasized the need to develop data in the severe accident area. This applies to both the behavior of fission products within molten metal fuel and the fuel 
extrusion process that is expected to terminate a potential HCDA.

- Most of the metal fuel data base from EBR-II does not cover the ternary metal fuel. Only in recent years has plutonium been added, and the corresponding data at higher burnups is only now becoming available.

\subsection{Research and Development Plans}

\subsubsection{Plans in Support of ALMR (PRISM)}

Much of the planned R\&D outlined in Section G.3.2.1 [Ref. 1] is an extension of ongoing work. Four items identified in Figure G.3.2-1 in the FY92 through FY95 time frame are noteworthy. The EM Pump Prototype testing and the Helical Coil Steam Generator Qualification Programs should provide data that will be important to future safety evaluations. In particular, the performance of the EM pumps is crucial during unscrammed events and any prototypic data would be scrutinized in detail. The third item, Licensing $R \& D$, is likely in reference to repeated requests for more data applicable to accident mitigation analysis. In particular, data on fuel behavior during rapid overpower events and on the behavior of fission products in molten fuel and sodium pools are needed. This may require a very substantial program. It appears that the applicant is simply indicating that they are working to develop a plan to obtain the necessary data. The fourth item, Metal Fuel Qualification is clearly part of a large ongoing effort. Therefore, we assume that where completion at the end of FY95 is indicated, they mean that is when the data base for prototypic $26 \%$ Pu metal fuel will be complete, i.e., that enough data will be available for all pertinent burnup levels.

In the longer term, the applicant is assuming that a prototypic unit will be built and ready for startup around the year 2000 . If the prototype is to be built and a set of tests is to be performed, it would be best if the NRC and its contractors were involved from the outset, as the system instrumentation will be crucial in acquiring all the data that may be required in the safety analyses of PRISM.

\subsubsection{Plans in Support of the Metal Fuel}

\subsubsection{Minor Actinides}

ANL is examining the impact of including minor actinides in the ternary metal fuel. If spent fuel from LWRs is to be used to make fuel for PRISM, and if a pyro-processing of that LWR oxide fuel is to be utilized, then the minor actinides are going to come along with the plutonium anyway. The advantage of consuming minor actinides in PRISM would be the transmutation of a key long-lived hazard that is currently slated to go into the repository. The disadvantage is that the minor actinides add to the decay heat load, change the reactivity feedbacks, and add further complications to the metal fuel chemical interaction with the clad. The fuel would become approximately $26 \% \mathrm{Pu} 1 \% \mathrm{~Np} 2 \% \mathrm{Am} .2 \% \mathrm{Cm} 61 \% \mathrm{U}$ $10 \% \mathrm{Zr}$ for the initial loading. Also, the minor actinides will tend to increase the sodium void reactivity worth and reduce the Doppler feedback, as is discussed in Section 4.5.4. In addition, there is significant uncertainty attached to the cross-sections for the minor actinides, so calculations regarding reactivity feedbacks and burnups would contain more uncertainty than currently is the case. It is noted that there are two proposed applications for "actinide burning" in metal fuel LMRs. The application proposed for PRISM is almost inadvertent, resulting from the fuel processing, and would result in a modest inventory of minor actinides. A more aggressive proposal is for a machine dedicated to minor actinide burning, which may be very different from PRISM and may not even come under NRC preview. 


\subsubsection{Fuel Cycle Facility: HFEF/South}

DOE and ANL continue to propose that metal fuel reprocessing facilities will be co-located with the reactors, although utility interest in owning and operating such facilities does not seem to be very high at this time. Still, a preliminary evaluation of such a facility by NRC is strongly desired by ANL and DOE so that the potential can be evaluated. The safety of such a facility is hardly a foregone conclusion, as there may exist issues such as fuel fires (if exposed to oxygen), and perhaps some remote chance of the inadvertent formation of a critical mass. ANL is working on a demonstration plant attached to EBR-II facility an its operation in the coming year should provide much insight into the matter.

\subsubsection{Integral Fast Reactor (IFR) Technology Demonstration}

Three Phases of the IFR program are described, with Phase II scheduled for completion at the end of FY90 and Phase III continuing through the end of FY95. Major accomplishments to be complete by the end of Phase III include: demonstration of high burnup fuel, demonstration of electro-refining on an engineering scale, provision of a "safety data base" in support of PRISM, converting the EBR-II core to $\mathrm{U}-\mathrm{Zr}$ and $\mathrm{U}-\mathrm{Pu}-\mathrm{Zr}$ fuel, and refurbishment of $\mathrm{HFEF} / \mathrm{South}$.

The Phase III outlined in this section involves several items that might be considered extensions of Phase II programs. However, by the completion of Phase III, the metal fuel cycle is to be "fully developed for commercial application." Several items are cited as being due for completion by the end of FY95:

- Fuel performance demonstrated to $150,000 \mathrm{MWd} / \mathrm{T}$ burnup

- Demonstration of "inherent safety potential" of "recycled fuels" through EBR-II

- Demonstration of fuel cycle on prototype scale

- Waste form certification

- Demonstration of actinide recycle capability

- Providing licensing data base support $w /$ NRC (of PRISM)

The first five items seem entirely consistent with discussions we have had with ANL staff members, and it seems likely they can complete or make major progress toward completing each of the five tasks. However, the significance of what is planned may be a little overstated. For example, it is already apparent that EBR-II is an excellent natural load-follow machine and that the passive reactivity shutdown will work well for unscrammed loss of flow and loss of heat sink events (there are questions about worst case UTOP events, but that is a different issue). ANL could likely load many variations on their metal fuel in EBR-II and continue to get the desired passive shutdown, but a major factor would be the small core with the core restraint system that is currently in place. Extrapolation to a larger PRISM core or any larger ALMR would require careful analysis.

The sixth item on the Phase III list could be interpreted to mean ANL's provision of technical support in discussions with the NRC, especially with respect to the behavior of the metal fuel in the PRISM reactor. However, some additional data for licensing interactions may also be required, and it is not clear how soon ANL will be able to develop such data.

\section{Key Issues/Concerns in Section 3.2}

- Inclusion of modest amounts (a few per cent, from LWR spent fuel) of minor actinides will have some impact on the reactivity feedback parameters and the reactor after-heat. However, a machine dedicated to "actinide burning" and containing more than $10 \%$ minor actinides would 
have very different reactivity feedbacks and may not retain the "passive shutdown" characteristic.

- Future tests to obtain "severe accident" data for metal fuel are going to be difficult and probably expensive. It may be prudent for the NRC staff to keep informed regarding the testing process.

\subsection{Other Apparent R\&D Needs}

Until very recently, most metal fuel work in the U.S. has been focused on improving the fuel burnup performance, demonstrating the fuel cycle, and optimizing some of the inherent safety capabilities in PRISM. A very modest effort was devoted to determining the metal fuel performance under severe accident conditions, i.e., during melts or power excursions. While the chances of such severe conditions developing in a metal fuel ALMR appear to be small, it is doubtful that a new reactor design would be accepted without well substantiated answers to some obvious questions. For the metal fuel ALMR, the questions are with respect to (1) the behavior of molten metal fuel, and its retention of fission products, while in the hot sodium pool, and (2) the behavior of the metal fuel during a power excursion, particularly the "extrusion" mechanism. 


\section{ANALYSES OF SAFETY ISSUES CITED BY GE IN APPENDIX G.4}

In Appendix G of Ref. 1, GE cited and addressed 19 safety issues originally raised by the NRC staff (and its consultants), either in the SER [Ref.2] or in discussions in technical meetings. Of the 19 issues and responses, some can only be evaluated by the NRC staff, some should be evaluated by BNL, in its consulting role, and some require evaluations by both. In this section, we will go through the issues in the sequence they appear in GE's Appendix G, offering detailed independent analyses in some cases, a few comments and remarks in others, and a simple comment deferring to the NRC staff where they are better qualified to evaluate GE's responses.

\subsection{Containment}

\subsubsection{Revisions to the Containment Design}

The containment concept originally developed for PRISM was unconventional, which is not surprising given the differences between PRISM and current light water reactors (LWRs) and even the more conventional liquid metal cooled reactors (LMRs). There are several contributing factors, including four that are particularly noteworthy. First, the sodium coolant system is at low pressure under normal operating conditions, so the need to use a large containment to absorb a massive loss-of-coolant accident is not present. Second, there are major advantages associated with keeping the core covered with sodium, so there is a strong desire to build a guard/containment vessel system to catch any leaks within a relatively small volume, so the sodium level won't fall too low. Third, with sodium in the reactor vessel and a moderate power production in the reactor, one can design a natural draft air-cooling system capable of removing after-heat, assuming one can indeed bring outside air into proximity with the reactor vessel. Fourth, when one down-sizes the reactor in order to achieve better safety characteristics, one has to minimize costs on a per reactor module basis, and the use of multiple large containment buildings could be prohibitively expensive. As a result, the applicant's original containment concept for PRISM bore little resemblance to those designed for conventional LWRs. (See also Section 6.2 of Ref. 1.)

In trying to convince the NRC staff that their original PRISM containment concept was appropriate for PRISM, the applicant relied heavily upon the argument that the chance of a major accident and radioactive release was very small. While these arguments had merits, there was and remains too much uncertainty regarding the metal fuel (among other things) for these arguments to be completely convincing. As a result the SER [Ref. 2] repeatedly identified concerns about various events that might lead to releases, and expressed concerns about the lack of an adequate containment for PRISM.

As a result of these concerns, the applicant chose to upgrade the PRISM containment. The revisions fall into three categories. First, they have added provisions that "ensure that none of the event-category III (EC-III) bounding events of concern leads to core damage or sodium boiling", in an attempt to limit the probability to less than $10^{-6}$ (1 chance in 1 million) per plant year. Second, they strengthened design provisions to ensure the vessel and vessel closure would resist core melts and possible HCDAs. Third, GE added a containment dome above the head access area (HAA) and added isolation valves in the IHTS, trying to assure that the probability of a $1 \mathrm{rem}$ radiation dose at the site boundary

over a 36 hour period following a severe accident is less than $10^{-6}$ per plant year. The revised PRISM containment concept is shown in Figure 1.

It is noted that the revised containment concept is still consistent with the four objectives pointed out above, i.e., it is still relatively small, which should lead to less expense, and consistent with natural draft air-cooling the vessel. Further, it may be the correct concept for the PRISM design, depending on 


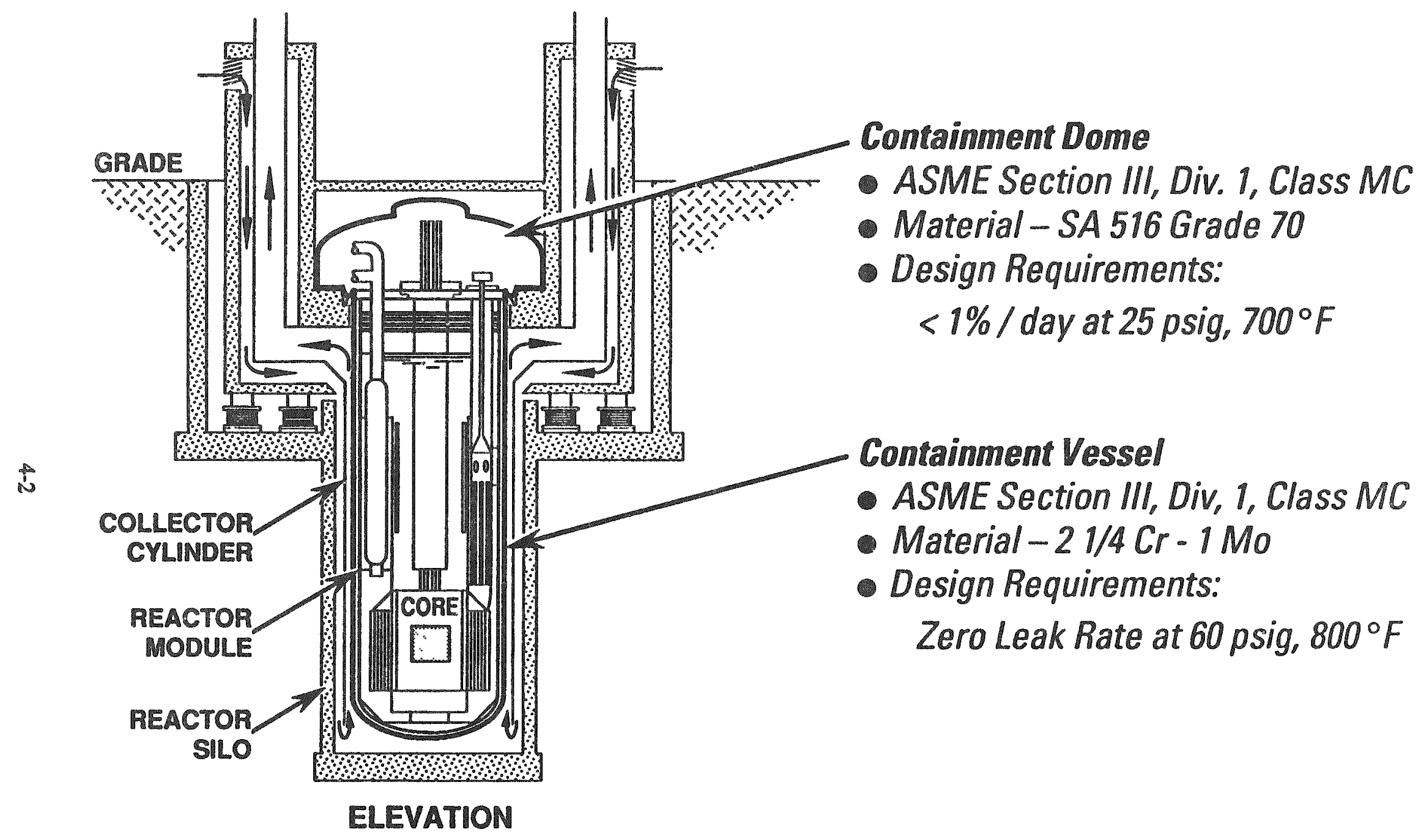

Figure 1. PRISM Containment Dome and Vessel. 
whether it could, in fact, withstand postulated HCDA events.

\subsubsection{Design Basis/Site Suitability Source Term}

While there is considerable agreement that a mechanistic source term would be preferable to the non-mechanistic source terms currently used for LWRs, it is difficult to come up with a reasonable source term for the ALMR (PRISM). Initially, the applicant argued that the chances of an HCDA or a core melt were so low that some minor release should be assumed as a design basis event. After considerable discussion, the applicant has now moved toward using a source term based on a substantial HCDA that triggers a major sodium fire in the containment dome.

While it is believed to be a positive development for the applicant to acknowledge that some worst-case events can not be entirely ruled out, the effort to analyze the mitigative capabilities of the design suffers from some major gaps in the data. First and foremost, without data on fuel extrusion for rapid power excursions it is very difficult to estimate when the excursion would be terminated. This would be difficult data to acquire, although there is currently a test program [Ref. 4] to develop similar data for oxide fuel. Second, the major factors preventing the release of harmful fission products will be (1) retention in the fuel due to its comparatively low melting temperature and (2) retention in the sodium pools of key fission products such as iodine. Again, there is relatively little data to support these arguments, even though it seems that such behavior would be likely.

The applicant defines the containment design basis in Table G.4.1-1, with the releases defined over the initial 10 seconds (the HCDA) and over the period from 10 seconds through 6 hours (the sodium fire). The significance of the 6 hour cut-off is not explained, although the containment pressure turns negative (relative to outside the containment) in this time frame, i.e., once the oxygen has been consumed in the fire. During the first 10 seconds, it is assumed that all of the noble gases are released, that $0.1 \%$ of the halogens (iodine), the alkali metals (cesium), tellurium and ruthenium escape, and that $0.01 \%$ of the strontium, barium, fuel, and other fission products are released. Over the longer interval, an additional $0.8 \%$ of the halogens, $1.6 \%$ of the alkali metals, $0.004 \%$ of the tellurium and ruthenium, $0.0016 \%$ of the strontium and barium, and $0.0008 \%$ of the fuel and other fission products are assumed to be released. Also indicated in this table is the release of $0.4 \%$ of the sodium over the 10 second to 6 hour period, presumably corresponding to the amount burned, and the assumed containment leak rate of $<1 \%$ day at $0.274 \mathrm{MPa}(25 \mathrm{psig})$ and $644 \mathrm{~K}(700 \mathrm{~F})$.

It should be noted that the processes involved can be very complex, and involve a combination of chemistry and radioactive decay. For example, the fission products Br-89 and I-138 are both soluble in sodium, and can be released from the fuel and transported efficiently in the coolant. However, each isotope decays within a few seconds, to $\mathrm{Kr}-89$ and Xe-138, respectively. These noble gasses will escape to the cover gas, before decaying a few minutes later to $\mathrm{Rb}-89$ and $\mathrm{Cs}-138$, respectively, which would likely settle out on some surface. Thus, the process by which the fission products can escape the fuel, the sodium pool, the cover gas, and perhaps even further is very complex, and a substantial effort may be required before a reasonably accurate source term can be developed.

The applicant does not explicitly state in Appendix $G$ what the basis is for the numbers in Table G.4.1-1, but it is very likely the basis is data from oxide fuel in sodium pools. If that is true, there are three problems related to the usage of such data to extrapolate for metal fuel. First, the models for oxide fuel are largely empirical, and there is little basis for extrapolating from equations which are mostly fitted data. Second, the metal fuel melts at a relatively low temperature so many fission products would remain in solid form, i.e., as solid particles in molten liquid fuel. As a result, they are more likely to stay with the fuel, which is helpful. Third, the metal fuel includes zirconium rather than oxygen, so the 
sodium-fuel chemistry would be different. As a further complication, if the initiating event is an HCDA, some of the fuel could reach very high temperatures, so a portion of the fission products could become molten or even conceivably vaporize. The net of all this is that the numbers in Table G.4.1-1 are very uncertain.

\subsubsection{Containment Performance Analysis}

Note: This section involves a check of the GE calculation in Appendix G.1, and entails several simple checks of the results presented by GE. Since GE's plots are all in "engineering units" rather that SI units, we depart from our normal practice here and sometimes use engineering units as the primary units and indicate the SI equivalents parenthetically.

GE sponsored calculations by the Westinghouse Hanford Corporation (WHC) to analyze the containment system performance during the proposed design basis event (the HCDA and sodium fire), using the CONTAIN Code [Ref. 5]. While Hanford staff members had experience using the CONTAIN Code for this type of application (for FFTF), they relied upon the CONTAIN Code developers at Sandia National Laboratory for up-to-date versions of the code, as well as updated input descriptions. (As it turned out, there was at least one instance where there was some confusion, and an incorrect data specification lead to what appears to have been a relatively small and conservative error in the CONTAIN results included in Appendix G.)

The CONTAIN Code was used to predict conditions within the containment dome and the quantities of the various isotopes released from the containment during the several hours of transient time analyzed. Since the CONTAIN Code does not include the capability to calculate the radiological consequences of any release from the containment, GE used the SMART [Ref. 6] Code to estimate the radiological consequences of the releases predicted by CONTAIN.

Because of the large uncertainties associated with both the size of the possible HCDA initiator and the behavior of the fission products in the fuel and in the sodium pool, we did not commit major resources to evaluating this event in detail. However, the impact of a large sodium fire on the containment is of interest and enough is known about the system design and the phenomena involved to make its analysis significant and credible. Therefore, some steps were taken to determine whether that portion of the CONTAIN analysis appears to be correct, what the impact on the containment might be, and how the predictions depend on key assumptions in the analysis.

\subsubsection{GE's Analysis of the Event}

GE provided a description of the assumptions used in specifying the system and the accident scenario for the CONTAIN calculation. A key assumption regarding the sodium fire is that the sodium pool is in direct contact with the containment atmosphere, which should give a maximum rate of burning. Several important parameters are listed in Table G.4.1-2, and seem to be at least approximately correct. The assumptions regarding fission product transport within the containment dome and the containment dome leak area (0.0005 square inches) were not evaluated for the reasons discussed above.

Results from the CONTAIN calculation for this design basis event are summarized in Figures G.4.1-7 through G.4.1-20, which include containment dome pressure, water vapor mass, containment cell temperatures, oxygen mole fraction, intercell flow rates, oxygen consumption rates, sodium combustion rates, combustion energy generation rates, structural temperatures, leak flow to the environment, as well as aerosol deposition and suspended aerosol with and without fire, as a function of time (minutes) into the event. Most of these plots go out 600 minutes (10 hours), although there are a 
few that terminate after 200 minutes when the inventory falls to zero fairly early in the event.

The plots indicate four major periods in the event, after the initial HCDA. During the first four minutes, or so, a rapid heat-up takes place, leading to peaks in the pressure and the leak rate to the environment. After about 50 minutes, the water vapor is exhausted. This shows up as a change in the slope of curves tracking the containment pressure, the leak rate, the cell atmosphere temperatures, and the sodium combustion rate. Between 4 minutes and 360 minutes, the sodium combustion process decays on an exponential basis (with the kink in the curves at 50 minutes, of course), as the cumbustion rate is proportional to the amount of reactants present and therefore decays exponentially. After the oxygen has been fully consumed (slightly past 6 hours), the whole process flattens out, with cell temperatures gradually steadying, and inter-cell flow rates leveling at just under $2000 \mathrm{lbs} / \mathrm{minute}$.

\subsubsection{BNL's Evaluation of the Applicant's Results}

\subsection{Total Energy Released by the Sodium Fire}

The most crucial factor in this analysis is the sodium combustion, which forms sodium monoxide Zwhen there is no excess oxygen available:

$$
2 \mathrm{Na}+1 / 2 \mathrm{O}_{2} \rightarrow \mathrm{Na}_{2} \mathrm{O}+2195 \mathrm{cal} / \mathrm{g} \text { 。 }
$$

and sodium peroxide if there is excess oxygen available:

$$
2 \mathrm{Na}+\mathrm{O}_{2} \rightarrow \mathrm{Na}_{2} \mathrm{O}_{2}+2500 \mathrm{cal} / \mathrm{g}
$$

The amount of energy released per unit mass of the reaction product is $3955 \mathrm{BTU} / \mathrm{lb}(9.2 \mathrm{MJ} / \mathrm{kg})$ for the sodium monoxide and $4505 \mathrm{BTU} / \mathrm{lb}(10.5 \mathrm{MJ} / \mathrm{kg})$ for the sodium peroxide. Since there is much more sodium available than oxygen, then it would seem that more sodium monoxide would form than sodium peroxide. If we compare Figures $\mathrm{G} .4 .1-12$ and -13 , which show the rate of oxygen combustion and the rate of sodium combustion, we can infer that for each pound of oxygen consumed, a little more than two pounds of sodium are consumed (this trend roughly holds true through the transient). This implies that approximately twice as much (moles) sodium monoxide is being formed as compared to the formation of sodium peroxide. Since we began with about $602(273 \mathrm{~kg})$ lbs of oxygen in the containment dome, this implies that about $1166 \mathrm{lbs}(529 \mathrm{~kg}$ ) of sodium monoxide and about $734 \mathrm{lbs}(333 \mathrm{~kg})$ of sodium peroxide are formed. This should release about 7.9 million BTUs of heat. If we integrate the area under the curve in Figure G.4.1-14, which shows the combustion energy generation during the transient, we get between 7 and 8 million BTUs. This indicates the CONTAIN calculation is at least consistent with respect to the chemical reactions.

As a conservative variation, we could assume that all of the oxygen goes to form sodium monoxide. This would create about 2333 pounds of sodium monoxide, releasing about 9.2 million BTUs of energy. Therefore, even if all the oxygen went into forming sodium monoxide, the increase in energy release would be only around $16 \%$, which would not appear to pose a major problem with respect to the apparent safety margins for this event.

\subsection{Rate of Combustion}

While the total energy produced by the sodium fire is important, it is the rate of combustion that directly impacts on peak temperatures and pressures within the containment dome. Models used for analyzing sodium pool fires are quite complex, as they must simulate the air and sodium flow patterns 
around the sodium-air interface. In addition. such models typically contain some input data that is somewhat judgmental in nature, so the user may have more control over key parameters ("dials") than might be desirable. However, there has been quite a bit of validation work done to confirm the performance of sodium pool fire models used to support the CONTAIN Code [Ref. 7]. Therefore, barring some user-input errors, the sodium fire analysis used in the applicant's simulation is probably fairly accurate.

As a further check for this combustion rate, we utilized a "rule of thumb" cited in Reference 8 , i.e., "Typical burning rates for pool fires in air are around $25 \mathrm{~kg} \mathrm{Na} / \mathrm{meter}$ squared/hour". Since the sodium pool diameter is 5.64 meters, this implies an initial energy release rate of about $2.16 \mathrm{MJ} / \mathrm{sec}$ (2048 BTU/sec), assuming that all the sodium goes to creating sodium monoxide. The peak combustion energy generation shown on Figure G.4.1-14 is about $1400 \mathrm{BTU} / \mathrm{sec}$, which reflects several geometric considerations that are not in our "rule of thumb" estimate. Due to the fact that this energy generation rate is not out of line with respect to our very rough estimate, and the fact that the sodium pool fire models used with CONTAIN have a fairly good validation base, it seems likely that Figure G.4.1-14 is at least approximately correct.

\subsection{2.3 Rate of Air Heat-Up}

The initial inventory of air in the containment is roughly $1180 \mathrm{~kg}$ (2601 lbs), and the heat capacity of air around $477 \mathrm{~K}(4 \mathrm{COF})$ is about $1028 \mathrm{joules} / \mathrm{kg} / \mathrm{K}$. At the original rate of energy generation of $1.47 \mathrm{MJ} / \mathrm{s}(1400 \mathrm{BTU} / \mathrm{s})$, the air in the containment would increase from $100 \mathrm{~F}$ to $550 \mathrm{~F}$ (see Figure G.4.1-9), i.e., by $450 \mathrm{~F}$ or $250 \mathrm{~K}$, in a little under 3.5 minutes. This then explains why the air temperatures in this figure increase so rapidly.

\subsection{The Pressure Increase Over the First 4 Minutes.}

The ideal gas law dictates the relationship between temperature and pressure, assuming the volume and mass hold constant. In this case, the pressure in $\mathrm{Pa}$ is equal to 325 times the temperature in $\mathrm{K}$. Thus, initially the pressure of $101325 \mathrm{~Pa}$ (1 atmosphere, $14.7 \mathrm{psia})$ is consistent with the temperature of $311 \mathrm{~K}(100 \mathrm{~F})$. Once the sodium fire heats the atmosphere to $561 \mathrm{~K}(550 \mathrm{~F})$, the pressure should reach approximately $183,300 \mathrm{~Pa}$ or $26.45 \mathrm{psia}$, assuming the amount of oxygen consumed by the fire can be neglected for the first four minutes. This pressure is about $11.75 \mathrm{psig}$, which is a little higher than the peak pressure of $9.8 \mathrm{psig}$ in Figure G.4.1-7 (which would include the consumption of oxygen in the fire).

\subsection{The Pressure Peak at 4 Minutes}

As the air in the containment dome is heated, some heat starts to transfer to the internal structures and through the dome to the outside air. Once that rate of heat transfer equalizes with the rate of energy being generated by the sodium fire, the air temperature will peak. With the dome having a heat transfer area of about 336 square meters and the equipment "slab" having a heat transfer surface area of about 107 square meters, the temperature required to release the energy from the initial portion of the fire would be about $544 \mathrm{~K}(520 \mathrm{~F})$. As was discussed in the previous subsection, a temperature of $550 \mathrm{~F}$ would give a pressure a little below $11.75 \mathrm{psig}$. Therefore, the temperature peaking around $550 \mathrm{~F}$, as shown in Figure G.4.1-9, is entirely reasonable.

It should be noted that the containment is designed to leak less than $1 \% /$ day at 25 psig $(0.274$ $\mathrm{MPa}$ ) and $700 \mathrm{~F}(644 \mathrm{~K})$. While we are making several approximations in comparing against the CONTAIN calculations, none of these approximations suggest errors large enough to increase the 
containment pressure and temperature nearly that high. Even if several worst case assumptions were combined, it seems unlikely that the design basis sodiun fire would push the containment pressure above 25 psig.

\subsection{The Pressure Decrease after 4 Minutes}

Once the heat transfer process catches up with and then passes beyond the heat generation rate from the sodium fire, the system temperatures can level off and begin to decrease as the fire starts to exhaust the supply of oxygen. In addition, as the oxygen portion of the air is gradually consumed, the pressure decreases. In fact, if we go back to the ideal gas law, and estimate the pressure of only the nitrogen portion of the air at the elevated temperature of $325 \mathrm{~F}$ ( $436 \mathrm{~K}$ ) (from Figure G.4.1-9 at 6 hours), we get .1048 MPA (15.2 psia). On the gauge scale this is $0.5 \mathrm{psig}$, which compares well with the containment dome pressure of 0.1 psig at 6 hours on Figure G.4.1-7.

While we can estimate where the pressure will go to, it is more difficult to confirm the trend through the 6 hours of transient. However, the dominant processes are rate dependent, i.e., the rate of change is proportional to the inventory. This should lead to classic exponential decay curves. In fact, most of the curves from the CONTAIN code calculations fit this description. The only oddity during this long period shows up as a kink in the slope (of several curves) after about 55 minutes. The reason for this kink can be traced to Figure G.1.4-8, which shows the water mass in the containment. If we assume $100 \%$ relative humidity in the containment at the start of the transient, with the ambient temperature of $310 \mathrm{~K}(100 \mathrm{~F})$, we can estimate a water vapor pressure of $6.632 \mathrm{kPa}(.96 \mathrm{psia})$ which gives a mass of $51.4 \mathrm{~kg}(113.3 \mathrm{lbs})$. Due to an error in the input deck, the initial water vapor inventory from the CONTAIN Code calculation, shown in Figure G.4.1-8, is $157 \mathrm{lbs}$, which is $39 \%$ too high. In either case, the water vapor reacts with the sodium to form sodium hydroxide:

$$
2 \mathrm{Na}+2 \mathrm{H}_{2} \mathrm{O} \rightarrow 2 \mathrm{NaOH}+\mathrm{H}_{2}
$$

This reaction acts to remove the water vapor from the containment atmosphere during the first hour. Once the water vapor has been removed, the rate of the drop in pressure slows, which creates the various kinks in the curves at 55 minutes. Because there is $39 \%$ too much water vapor in the CONTAIN calculation, the impact and duration of this process is exaggerated somewhat. However, there is far more oxygen and nitrogen in the containment atmosphere, so a $39 \%$ error in water vapor inventory is probably not very significant.

\subsection{Summary Assessment of CONTAIN Calculation}

Our various attempts to probe the results from the CONTAIN calculation indicate that the analysis seems reasonable. The user-input mistake in specifying the amount of water vapor in the containment dome is the type of error that occurs fairly frequency when a large computer code is being modified, and is unlikely to have changed the results enough to cause concern. Our simple analysis shows there are large margins to accommodate errors and uncertainties, in that the pressure increase could more than double before there would be any cause for concern. Therefore, our best estimate at this time is that the PRISM containment dome could accommodate a worst case sodium pool fire, and would do so with fairly large safety margins.

With respect to the fission product release fractions and site boundary doses, we feel there is far greater uncertainty involved. In Table G.4.1-4, the applicant shows projected doses at the site boundary ranging from $1 \%$ to $40 \%$ of the Protective Action Guidelines (PAGs). However, these low doses are due to the relatively small amount of fission products and fuel that escape from the sodium pool into the 
containment dome (see Section 4.1.2).

The data base supporting those release rates is not complete, so these predicted doses involve a great deal of angineering judgement and should be used with care. A major concern here is that the models for fission product release from oxide fuel are largely empirical, so the extrapolation to metal fuel is based on observation more than understanding. However, GE/ANL arguments regarding the likely retention of fission products within the metal fuel and the sodium pools appear to be a reasonable assumption.

\subsubsection{Other Events Covered in Appendix G.4.1}

The applicant provides a brief description of some other events that might result in a leakage into the containment. This list includes a maintenance related opening in the reactor closure, a large primary sodium spill, a major refueling accident, and a couple of postulated IHX failure events (which challenge the isolation valves added to the IHTS). In most cases, the applicant uses qualitative arguments regarding either the likelihood of such events or the probable outcome of such events. As these events seem far less significant than the design basis event previously discussed, we spent little time or effort reviewing these other events.

Key Issues/Concerns in Section 4.1

- The current PRISM Containment Design appears to be appropriate for that design, and could probably accommodate a postulated HCDA event. The remaining concerns are regarding the severe accident data base for metal fuel, which is relatively limited at this time.

\subsection{Shutdown System}

In the original draft SER [Ref. 2], several concerns were expressed about the capability of the passive ("inherent") shutdown system, which is based on reactivity feedback characteristics, to adequately serve as a second reactor shutdown system. In particular, the large positive sodium void reactivity worth and the potential for reactivity additions should fuel motion occur, made this a particularly important question.

The applicant made two significant changes to the PRISM Reactor Shutdown System, i.e., the addition of rod stops on the primary control rods and the addition of the Ultimate Shutdown System (USS). The rod stops became necessary when new data on the ternary metal fuel and a closer examination of the variations during the fuel cycle indicated that the burnup reactivity swings could no longer be limited to around 35 cents, thereby raising the possibility of a reactivity insertion caused by control rod withdrawal too large to be accommodated safely without scram. The USS device was added to resolve the question of how they intend to eventually terminate long term unscrammed event.

\subsubsection{The Control Rod Stop System}

This system is comprised of a motor driven, movable (mechanical) stop within each control rod drive mechanism and a computerized controller. The stop physically limits the withdrawal stroke of the control rod drives, as indicated in Figure 2. An electronic controller computes the position to which the rod stop should be set, subject to plant operator permission for set changes, in order to accommodate reactivity changes over the operating cycle. It is expected that resetting the rod stop position will be required 5 to 6 times each 18 month fuel cycle. The requirements include limiting the possible reactivity insertion to $40 c$ (including a $10 c$ margin for uncertainties) when all rods are withdrawn, allowing for 
DRIVE MECHANISM

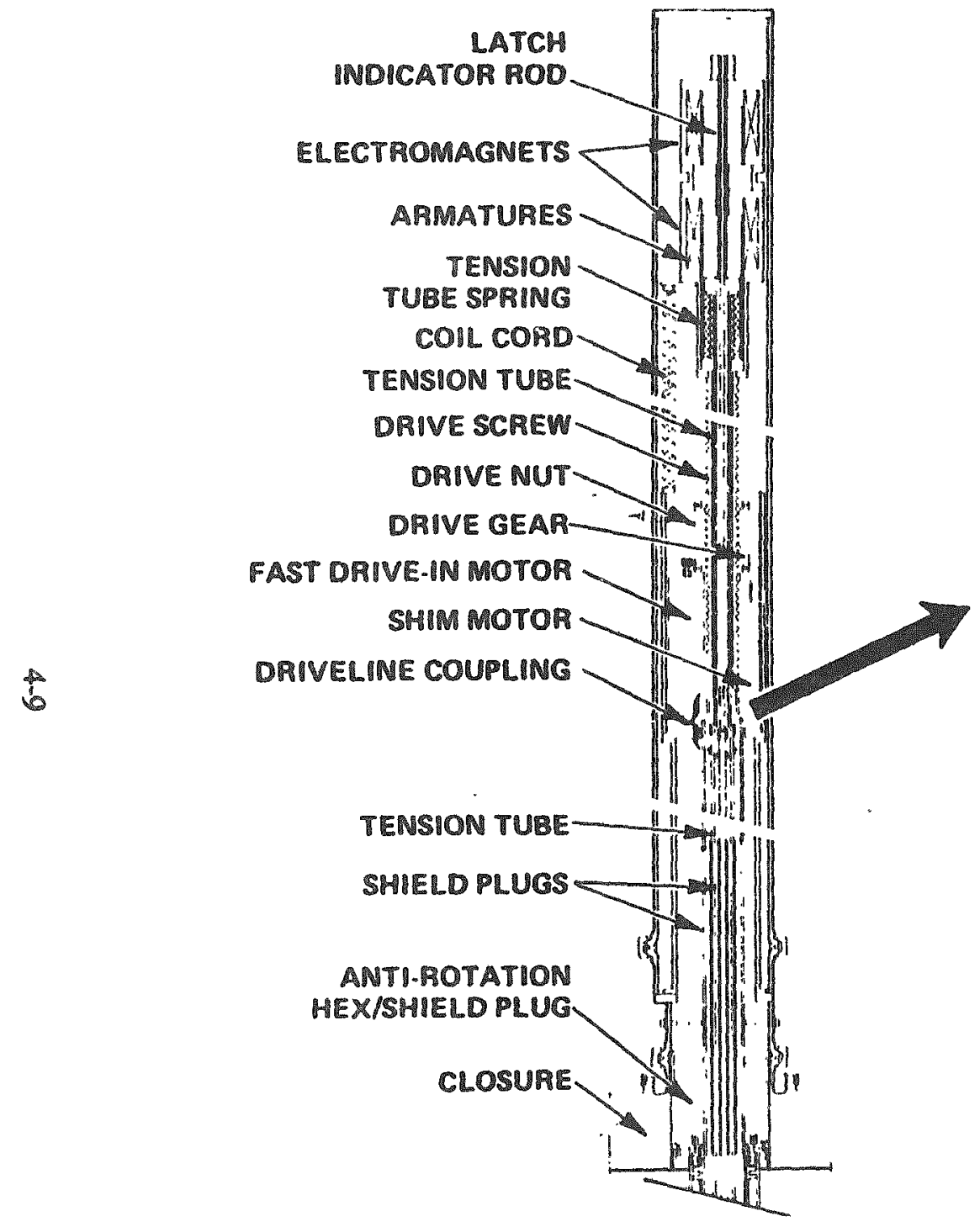

ROD STOP MECHANISM

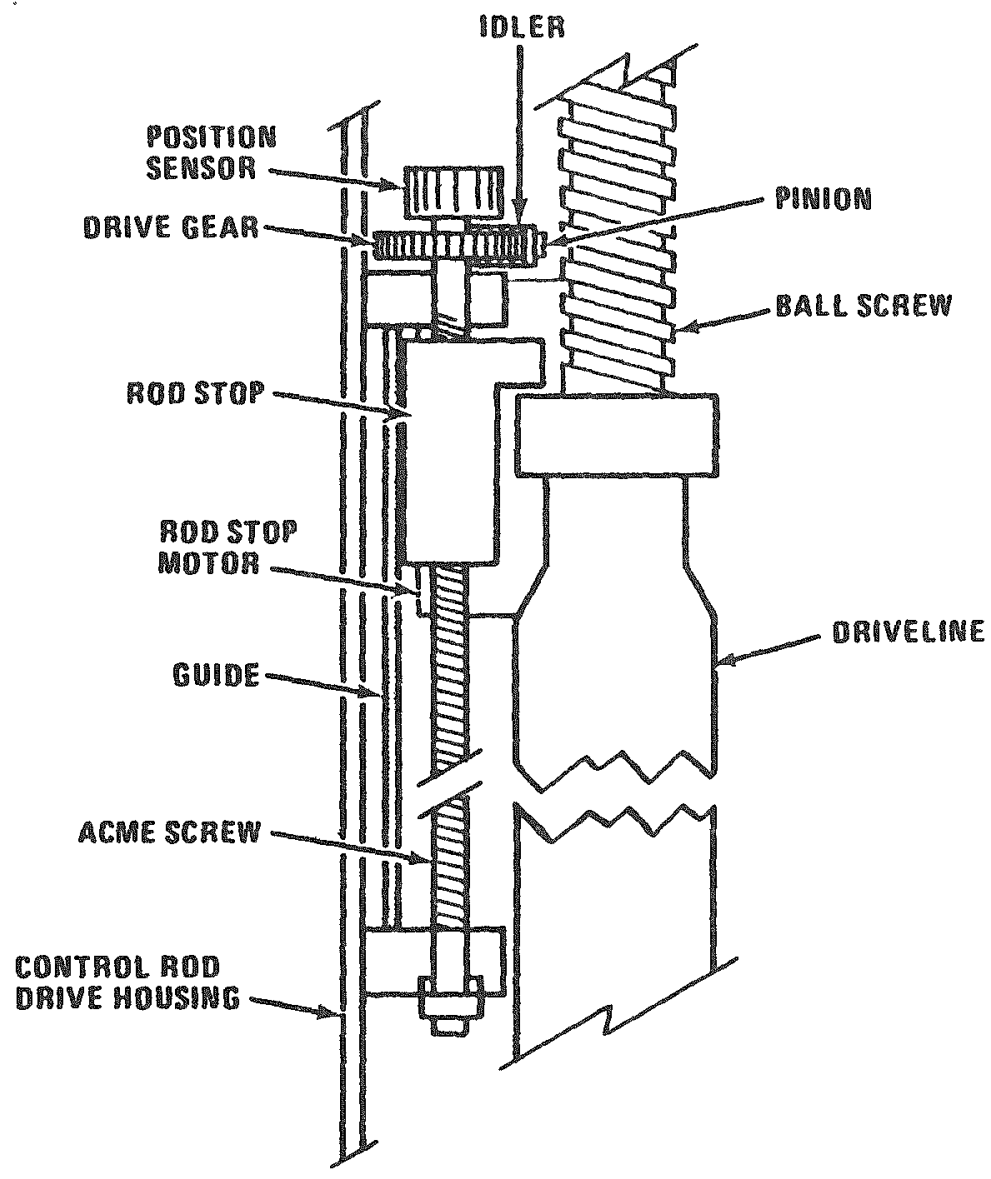

Figure 2. Control Rod Drive and Rod Stop Mechanism. 
adjustment during the operation cycle, and having a probability of failure on demand of less than one-in-a-thousand.

At the beginning of cycle, the core reactivity is highest and the control rods are inserted the furthest, inserting perhaps a dollar or more of negative reactivity. At this stage, the mechanical rod stops would be positioned fairly low so the top of control rod drive lines would butt up against them before the rods are withdrawn too far, i.e., more than $40 \mathrm{c}$, including uncertainties. After several weeks, the core reactivity will have decreased and the control rods will have been pulled out to compensate. Eventually the top of the control rods will approach the rod stops, at which time the rod stops would have to be moved higher to allow the control rods to be further withdrawn. Ideally, each raising of the rods stops would take place when the maximum reactivity insertion is only a few cents, and after the adjustment the maximum possible withdrawal would be around 30 cents.

The rod stops became necessary once it was determined that 5\% axial swelling (with burnup) would require at last $\$ 1.20$ of reactivity compensation (rod withdrawal). With the rod stops becoming necessary, new failure modes become apparent, e.g., someone or something could raise the stops too high, and a reactivity insertion of greater than $50 \mathrm{c}$ could be possible, and such a large insertion could result in fuel damage.

Because the rod stops must be moved regularly, the question of precision becomes important. At the mid-core position, the withdrawal of all six control rods one inch inserts a maximum of 76 cents of reactivity. If we assume the applicants estimates of measurement errors of less than .01 inches, there is relatively little reactivity uncertainty from measurement error. However, the uncertainty associated with predicting the reactivity worth of the rods at the new maximum withdrawn position could be significantly larger.

\subsubsection{The Ultimate Shutdown System (USS)}

The USS is illustrated in Figure 3. $\mathrm{B}_{4} \mathrm{C}$ balls are stored in a dry canister above the core, and upon actuation, the balls fall by gravity down a guide tube into an open thimble at the core center. The reactivity worth of the balls is believed to be high enough to bring the reactor from $135 \%$ full power (UTOP) to cold shutdown.

The system is made up of four subassemblies, including the canister, the center shutdown absorber assembly, the absorber guide tube, and the guide tube drive mechanism. The canister keeps the absorber material dry and out of the core during normal conditions, and holds about one cubic foot of the $1 / 4$ inch diameter absorber balls. The canister release mechanism is operated by a drive motor at the top of the assembly, which pulls on a rod running the length of the canister. Absorber release is accomplished by pulling on the actuator rod and disengaging the pull pins. A backup release is available by pushing down on the actuator rod and shearing the hinge support bar. The center core assembly maintains the $B_{4} C$ absorber balls in the proper location once the USS has been actuated. The USS is activated manually from a pair of ultimate shutdown switches located in the remote shutdown facility, or from a similar pair of switches in the reactor protection system vaults.

Because the $\mathrm{B}_{4} \mathrm{C}$ balls are dropped into sodium within the absorber assembly, there could be some question about the balls falling into the core. The key consideration is the amount of sodium upflow in the assembly, which GE indicates should be quite low (roughly $.3 \mathrm{~kg} / \mathrm{s}$ ). At that low flow rate, the upward drag force on the $\mathrm{B}_{4} \mathrm{C}$ sphere should be much less than the gravitational force pushing downward. We've estimated that a sodium uplow of $11.8 \mathrm{~kg} / \mathrm{s}$ would be needed before the upward force would equal the gravitational of force. Therefore, unless the sodium flow through the USS assembly is roughly 40 


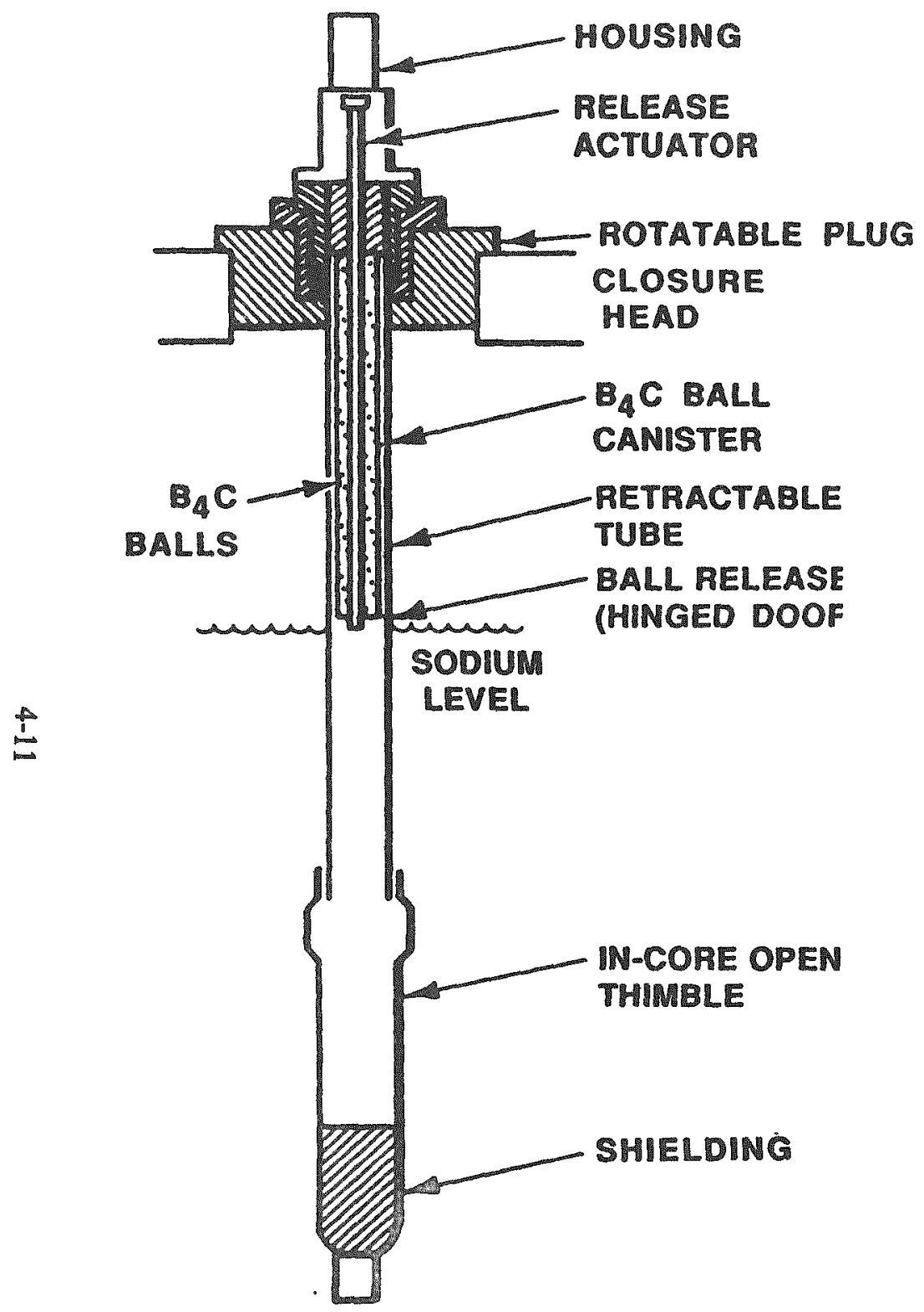

\begin{tabular}{|c|c|}
\hline \multicolumn{2}{|c|}{ CHARACTERISTICS } \\
\hline SHUTDOWN: & METAL \& OXIDE CORES \\
\hline DIVERSITY: & FULLY \\
\hline TESTABILTV: & TESTABLE \\
\hline RESETABULIT: & OFF-LNE \\
\hline ACTUATION: & MANUAL \\
\hline & - CONTROL ROOM \\
\hline & $\begin{array}{l}\text { - REMOTE SHUTDOWN } \\
\text { FACILITY (IE) }\end{array}$ \\
\hline
\end{tabular}

Figure 3. Ultimate Shutdown Assembly. 
times greater than the design level, the balls will fall down into the core.

The USS fulfills a necessary function, providing an alternate means of shutting down the reactor. The complexity of the USS design seems to be related to requirements including testability, internal diversity, reliability, and the ability to move it out of the way for refueling and maintenance. It is entirely possible that the device will provide the highly reliable backup shutdown capability that has been needed.

Key Concerns in Section 4.2

- Movement of the control rod stops must be fairly precise to minimize potential UTOP initiators. The two key factors are measurement errors, which should be small at a rate of $20 \mathrm{c}$ per inch for all 6 rods, and calculational error, which could also be minimized if one is careful to factor in the most recent data, e.g., from the most recent control rod movement.

- The USS provides the second shutdown function and allows the operators to terminate an unscrammed event. However it is a relatively slow system, and does rely on the reactor "passive shutdown" response to keep the reactor conditions acceptable for the first few minutes.

\subsection{0-Year Plant Life}

There are no known weaknesses in the PRISM design that would preclude an extended plant life-time, assuming that the necessary/studies are performed and key components are replaced when necessary.

Key Issues/Concerns in Section 4.3

None

\subsection{Seismic Isolators}

The principal issue regarding the seismic isolators is the need for further data, and the current requirements in this area can be better addressed by the NRC staff. Certainly this appears to be a promising area of study, but it will take time to develop a strong data base. With U.S. tests at ETEC and the University of California at Berkeley, and Japanese tests at CRIEPI and elsewhere, the data base is growing and could be fairly extensive before any PRISM prototype is built. The isolators planned for usage in PRISM are fundamentally simple and provide the desired horizontal isolation, which is particularly useful for the tall and narrow PRISM design.

Key Issues/Concerns in Section 4.4

None

\subsection{Sodium Void}

In this section, the applicant responded to concerns expressed in the SER [Ref. 3] and elsewhere regarding the large positive sodium void reactivity worth. This provides a potential means of accidentally increasing the core reactivity to up to nearly 6 dollars super critical, although realistically, the other reactivity feedbacks would respond fast enough to reduce the net reactivity. Regardless, a very damaging power excursion would likely result from a complete voiding of the core, so the positive sodium void 
worth is potentially a major safety concern.

The response provided by the applicant is similar to the previous position, although bolstered this time by an ANL study examining some trade-off options. Briefly, the applicant's position is that 1) wide-scale sodium boiling is highly unlikely, 2) that should such an event develop the energy could be absorbed in the vessels and structures, and 3) available options for reducing the sodium void worth are very unattractive and tend to cause problems in other areas. In the following sections, we'll consider each of these three response positions.

\subsubsection{Sodium Boiling is Highly Unlikely}

Because of the highly diverse reactor shutdown system and the reactivity-feedback based passive ("inherent") reactor shutdown mechanism, it certainly appears that wide-scale sodium voiding is indeed very unlikely to occur in PRISM. For an independent PRA study, it seems likely that a failure probability in the range of 1 in 100,000 would be assumed for the shutdown system. While this failure probability is more pessimistic than GE's, it is more optimistic than failure rates assumed for LWRs. Similarly, the failure rate to be assigned to the passive shutdown is probably in the range of 1 in 100 to 1 in 1000 attempts. Factored together, PRISM should eventually be credited for a failure probability in the range of 1 in 10 million to 1 in 100 million, per scram attempt. Since PRISM is relatively small, it could take 1000 reactor modules to eventually replace the current U.S. LWR capacity, and each module could be scrammed as frequently as 10 times per year (for the first few years, anyway). Over a 10 year period, that works out to 100,000 scrams. Therefore, over that 10 year period, the chance of a sodium voiding event occurring in a PRISM reactor might be between 1 in 100 and 1 in 1000 . This means that while a sodium voiding event is very unlikely, it is not impossible.

The arguments stated above assume that these systems perform as designed. There may be ways for the maintenance and operations staff to adversely impact on both the reactor scram system (maintenance) and the passive shutdown (synchronous machines), so the chances of sodium voiding is probably somewhat higher than the odds cited above. That is not to say that sodium voiding should be expected, because the defenses against such a development are indeed extensive. However, it is only prudent to consider the possibility of such a development.

\subsubsection{Can the HCDA Be Contained in the Vessel?}

There is a reasonably good chance that a large HCDA could be accommodated in the PRISM reactor vessel, but it may be some time before that can be established with confidence. Some essential data regarding fuel expulsion during rapid transients simply do not exist. The fastest data available are for an 8 second period transient-over- power event. In contrast, data for oxide fuel are available over a few millisecond period [Ref.4].

Because of the lack of the key metal fuel data, the applicant adapted some HCDA analysis that was performed previously for the FFTF reactor, and evaluated how the PRISM vessels and structures would stand up to that event. Their analysis indicates that the PRISM reactor vessel and closure can safely accommodate HCDA loads resulting from energetics on the order of $500 \mathrm{MJ}$ without loss of structural integrity, disengagement of the rotatable plug from the reactor closure, or expulsion of sodium. Independent (BNL) examination of the applicant's analysis has not revealed any apparent errors. Therefore, it does appear likely that the PRISM system could withstand this large HCDA postulated for FFTF. 
In comparing the postulated HCDA $s$ for different fuel types, a recent paper [Ref. 9] from the Indira Gandhi Center for Atomic Research is particularly useful. Their focus is on worst-case scenarios, with arbitrarily large reactivity insertions in $500 \mathrm{MWe}$ sodium cooled reactors, using metal, oxide, and carbide fuel. They make several interesting points:

- The lower operating and melting temperatures for metal fuel decreases any concerns about fuel-coolant interactions (FCIs), which are essentially benign for metal fuel.

- The large difference between the melting and boiling temperatures in the metal fuel will tend to keep the core together longer, and would result in larger melt fractions (perhaps $100 \%)$.

- Because the fraction of metal fuel melting is much higher, the potential reactivity insertion is metal fuel (due to slumping) is also higher.

- For reactivity insertions under about $\$ 75 /$ second, the metal core releases more energy than the oxide and carbine cores. However, for insertions above $\$ 100 /$ second, the energy release for the metal core is significantly lower.

Perhaps the most crucial finding from Reference 9 is that the energy release from a $\$ 200 / \mathrm{sec}$ ramp is only about $300 \mathrm{MJ}$, and is increasing only gradually as the reactivity insertion rate increases. For the $155 \mathrm{MWe}$ PRISM core, the release would be less, and certainly less than the $500 \mathrm{MJ}$ estimate made for FFTF and utilized by the applicant for determining HCDA loads. Thus, the analysis in Reference 9 supports the applicants ${ }^{\circ}$ contention that the PRISM vessel and head could survive a large HCDA event.

Finally, late in the review process it was revealed that the GEMs might expose the core barrel to relatively high fluence (the gas does not scatter the neutrons as the sodium would). This raises the possibility of radiation damage weakening some of the structure needed to withstand an HCDA. Although not a major concern at this time, the possibility of radiation induced embrittlement of structures should be considered in future evaluations.

\subsubsection{Can the Sodium Void Worth Be Reduced?}

\subsubsection{Options Considered by ANL and GE}

In response to requests from the various parties involved in reviewing and evaluating the PRISM reactor, ANL performed a fairly extensive study of options for reducing the reactivity worth from sodium voiding to $\$ 1$ or less, and some of this work is summarized in Appendix G.5. The choice of "less than $\$ 1^{\prime \prime}$ comes from the belief that such a reactivity addition could be countered quickly by Doppler and thermal expansion mechanisms, and the core would behave more like a partial core melt than a HCDA. Ideally, a negative sodium void reactivity worth would be best, but this would be extremely difficult unless the reactor was nearly as small as EBR-II.

While much of the ANL study on ways to reduce the sodium void worth in metal fuel LMRs was largely an update of some work compiled over the years (others have struggled with this problem before), it was fairly extensive. There are indeed a few options for reducing the sodium void worth, but they all seem to have serious adverse impacts. A major shortcoming in one of the more practical alternatives is that of spoiling the geometry enough to make the void worth small or negative. The burnup reactivity swing becomes quite large, which requires far more control rod compensation, which increases the 
magnitude of potential UTOP initiators. Thus, one can overcome the void worth problem, but obtains a large and potentially damaging transient-over-power initiator in the exchange.

\subsubsection{Alternatives Not Considered by ANL and GE}

It was implicitly assumed by ANL and GE that the metal fuel was the only fuel option to be considered. This is not an unreasonable assumption for them to make, as they have dedicated a major effort into developing the metal fuel and incorporating it in the PRISM design. The metal fuel has some very desirable characteristics, and the designers have implicitly assumed that the problem of a large positive sodium void worth is less important than the advantages gained elsewhere by using the metal fuel. Even so, there exists an oxide core design for PRISM, particularly for use in the international market. In addition, other fuel forms are being considered by the French and the Soviets.

While the ANL study did not consider alternate fuel types, there have been a few recent studies in which metal fuel was cross-compared against oxide, nitride, and carbide fuels [Refs. 10-14], including one in which ANL participated. When taken together, these studies show that metal fuel gives the largest positive sodium void reactivity worth, by at least $35 \%$ in comparison to the alternate fuel types.

Sodium reactivity worth estimations from two related studies for large LMR cores are shown in Figure 4A. In the ANL study [Ref. 10] for a large 3500 reactor, the sodium density was $35 \%$ higher in the metal fuel core than in the equivalent oxide fuel core. For a similar core, KfK [Ref. 11] estimated the sodium density feedback would be $38 \%$ higher with metal fuel than with oxide fuel. KfK also evaluated the Super Phenix core, and found that by substituting metal fuel for oxide fuel they would increase the positive sodium density reactivity feedback by more than twice (106\%).

In a recent study [Ref. 12], Westinghouse Hanford Corp. (WHC) has evaluated the potential impact of substituting nitride fuel into the PRISM reactor. Results regarding the sodium void reactivity worth are shown in Figure 4B. Using analytical tools based on neutron diffusion theory, WHC estimated a $70 \%$ reduction in sodium void worth. They then utilized analytical tools based on the more accurate neutron transport methods, and estimated that a $58 \%$ reduction in void worth could be gained by substituting nitride fuel into PRISM. In addition, WHC analyzed a shortened ("pancaked") PRISM reactor $76 \%$ as tall as the current design, and estimated the sodium void worth could be reduced to about 58 cents. In addition to work WHC performed in evaluating the nitride fuel effect on sodium void worth, they also simulated the key unscrammed events postulated for PRISM, i.e., the ULOF and UTOP, and found results with nitride fuel that appear to be better than those projected for the metal fuel.

The Soviets have also been considering alternative fuel types for incorporation in their $\mathrm{BN}-1600$ LMRs [Ref. 13]. A plot showing the relationship between four fuel options, i.e., metal, carbide, oxide, and nitride, for varying core heights, is reproduced here as Figure 5. This plot indicates that the metal fuel sodium void worth is considerably more positive (at least $50 \%$ ) than the other three fuel options. The nitride fuel was estimated to give the smallest sodium void worth, although not significantly better than oxide fuel, judging by the plotted results.

As was mentioned before, there is a strong trade-off between sodium void reactivity worth and burnup reactivity swing, and it is difficult to reduce both simultaneously into desirable regions. This is shown in Figure 6, which was adapted from Reference 12. The target zone indicated includes a negative or small positive sodium void worth and a burnup reactivity swing below $\$ 1$. As ANL has shown, it is difficult to develop a practical metal core with both a small burnup swing and a small sodium void worth, and this is supported by the metal fuel curve in Figure 6 which does not closely approach the indicated 


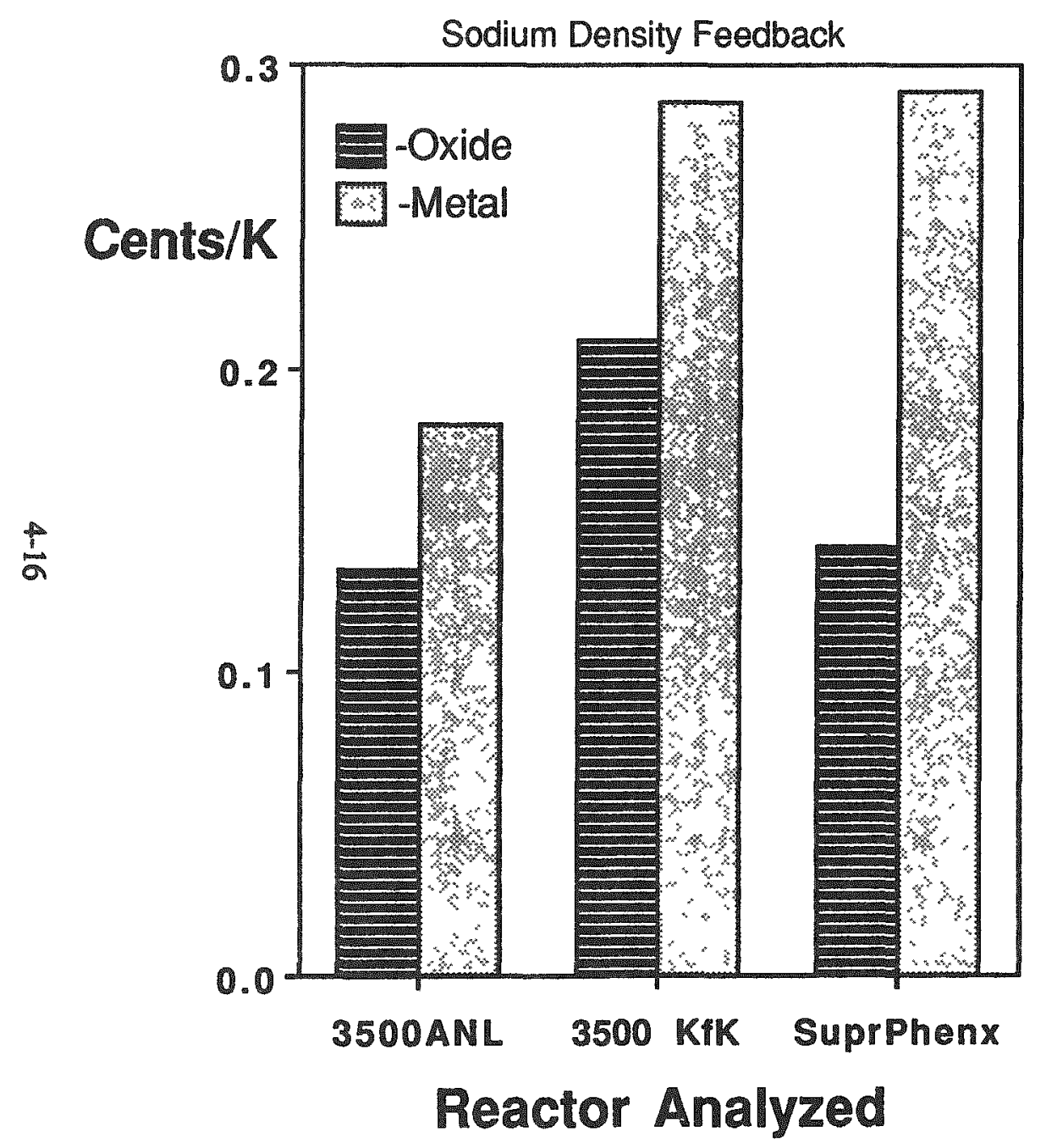

Figure 4a. ANL and KfK Studies in Proceedings of 1990 Fast Reactor Safety Meeting: by Cahalan, et al, and Royl, et al, respectively.

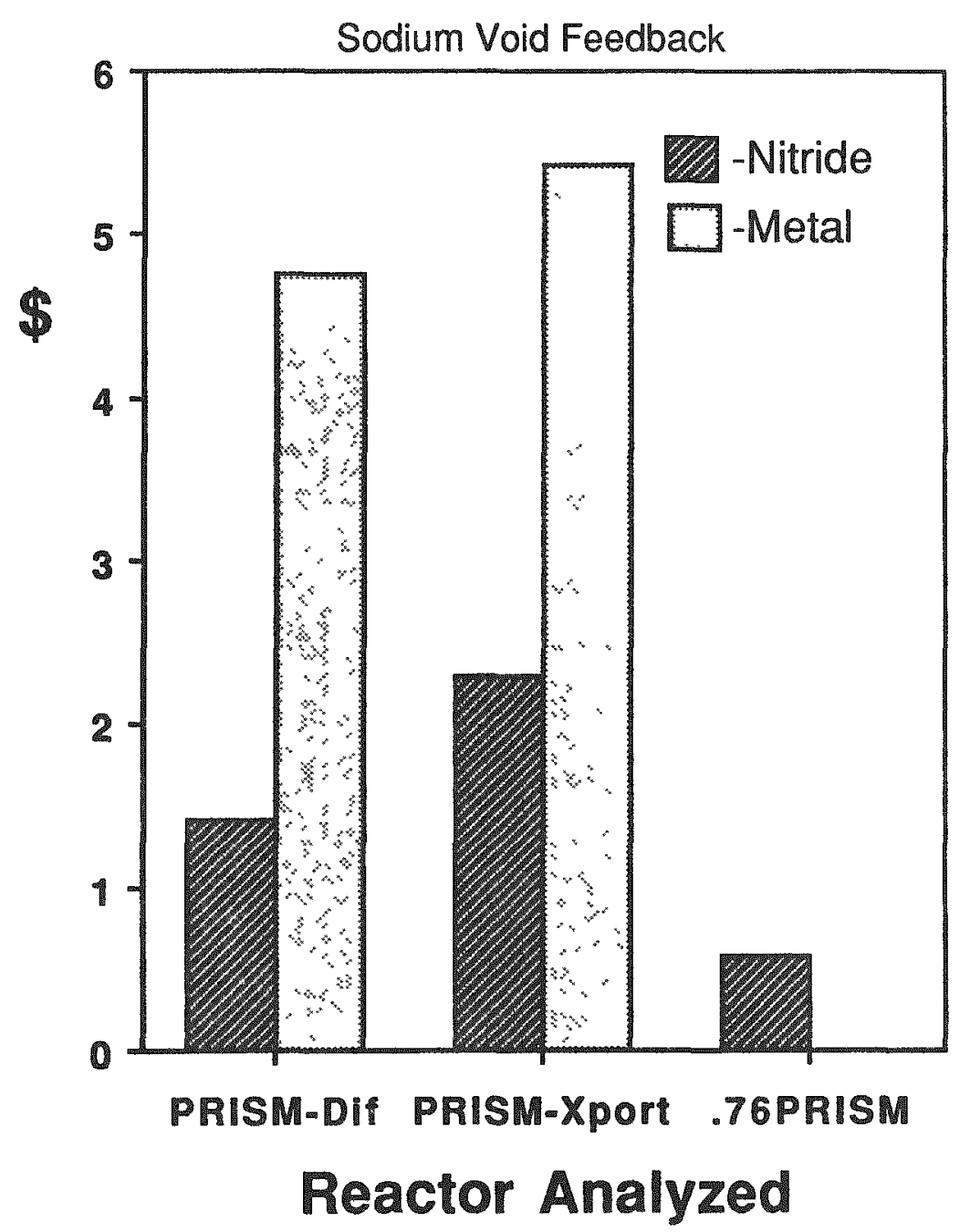

Figure 4b. Results from October 1990 Study of Omberg and Waltar for PRISM Core, Using Diffusion Codes and Neutron Transport Codes. 


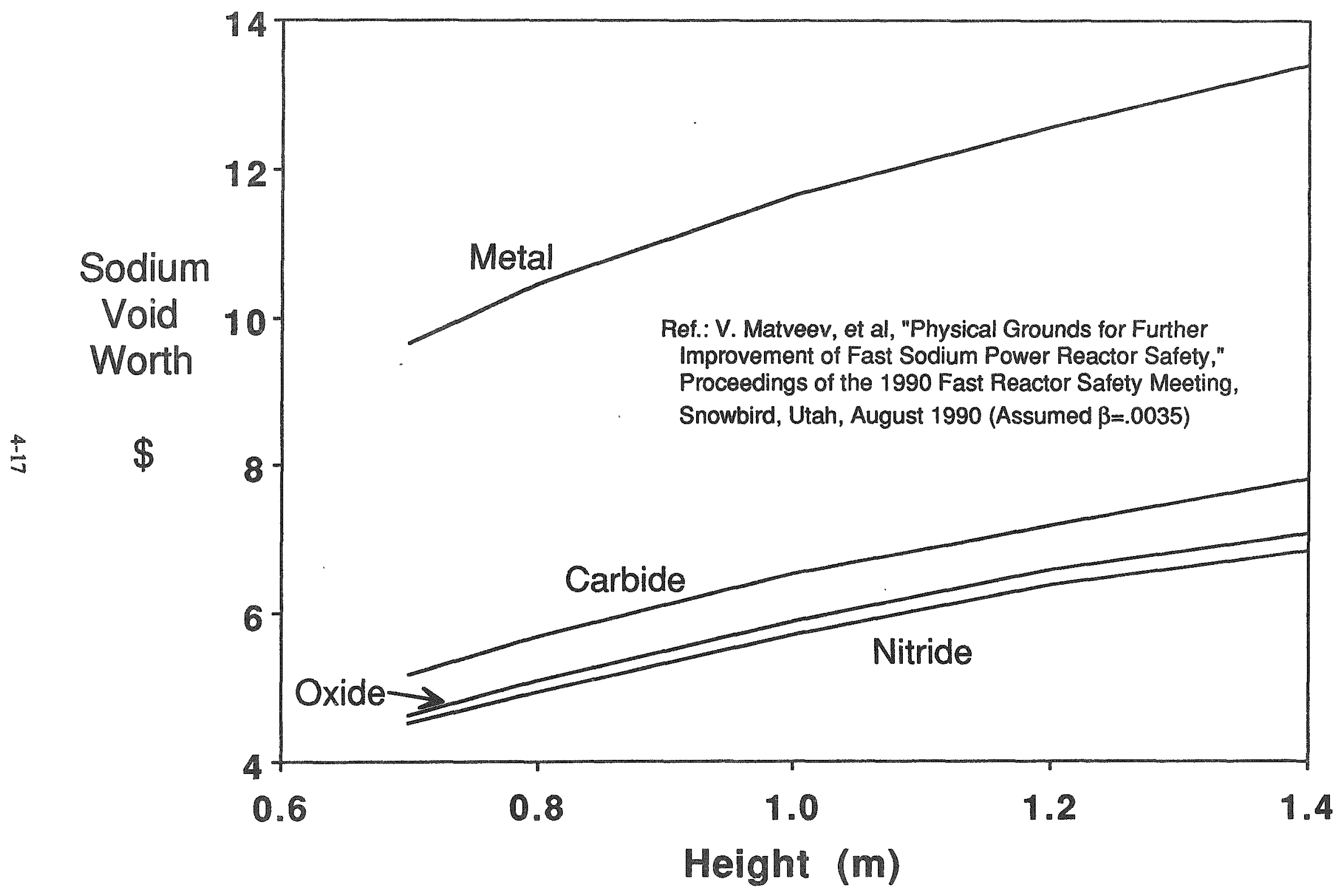

Figure 5. Effect of Core Height on Sodium Void Reactivity Worth for Four LMR Fuel Forms in Soviet BN-1600 Type Reactor. 


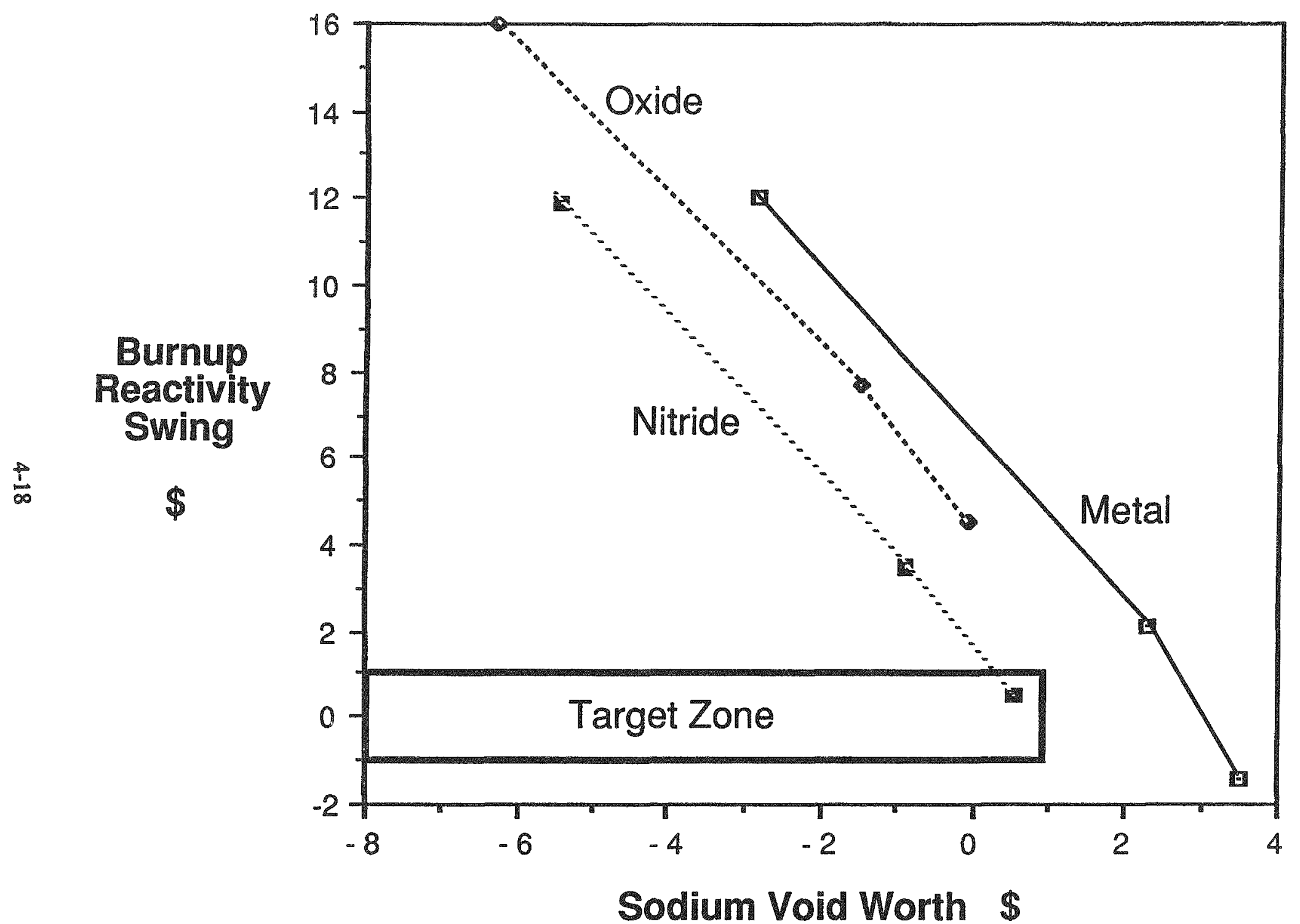

Figure 6 Trade-Off Between Sodium Void Worth and Burnup Reactivity Swing for a Small ALMR Core.

(Source: Comparative Sodium Void Effects for Different Advanced Liquid Metal Reactor Fuel and Core Designs", by K. D. Dobbin, et al., Proceedings of the International Conference on Fast Reactors and Related Fuel Cycles, October 28 - November 1, 1991, Kyoto, Japan). 
target zone. The oxide fuel curve is based on fairly high reactivity swings and negative sodium void worths, and does not extend to near the target zone. However, it appears the oxide fuel curve would narrowly miss this target zone if extended, although the oxide fuel curve would still be preferable to the metal fuel curve. The curve for nitride fuel includes a point within the target zone, with a sodium void worth of $57 \mathrm{c}$ and a burnup swing of $54 \mathrm{c}$.

Obviously, one does not select a fuel type based on a single characteristic, such a the sodium void reactivity worth. DOE sponsored parallel efforts for both oxide and metal cores in PRISM for the first two or three years of the program. A major consideration in DOE's commitment to metal fuel was the passive reactor shutdown, which is considered a major safety advantage that may well outweigh the disadvantage of the larger positive sodium void worth. It is not known whether nitride fuel was ever seriously considered for incorporation in PRISM.

There were four summary papers [Refs. 15-18] recently published which offer some brief comments regarding the work on nitride fuel. According to Ref. 18, the European Community, Japan, and the Soviet Union are all pursuing nitride fuel, since it has "high thermal conductivity, low fission gas release, low swelling, no cladding/chemical interaction" and is "compatible with the fuel system". According to Ref. 16, nitride fuel has "good irradiation behavior and straightforward reprocessing", along with its "better (than oxide fuel) heat transfer characteristics and higher fissile atom density". In Ref. 15, the authors concede that "nitride fabrication has been considered to be a difficult and expensive preparation", but claim that "this difficulty has been overcome: mixed mononitride pellets, with characteristics suitable for irradiation testing in the Phenix fast breeder reactor have been obtained in a classical oxide production facility without any significant modification of equipment". However, as cited in Ref. 17 , the available fuel performance data base for nitride fuel is relatively small.

While the nitride fuel appears to have a major advantage in terms of sodium void worth, and may perform at least as well as metal fuel regarding the passive reactor shutdown response, this fuel form is not well known in the U.S. The fuel development work outside the U.S. might eventually make nitride fuel a more practical option and needs to be watched. Regardless, if one wishes to evaluate the full spectrum of alternatives in trying to work around the sodium void worth problem, one could consider some of the alternate types of fuel that might be available.

\subsubsection{The Minor Actinide Factor}

Because most of the minor actinide isotopes (neptunium, americium, and curium) are far more likely to fission in a fast neutron spectrum, the inclusion of minor actinides in the fuel (which already includes uranium and plutonium) increases the sodium void worth. This is because the spectral hardening is much more helpful than the increase in neutron leakage is harmful. This preference for fast neutrons also impacts on the Doppler coefficient (fuel temperature). Without the minor actinides, the primary result of higher fuel temperatures is greater neutron capture in the "resonance" intervals. With the minor actinides, the increased fissioning in the minor actinides tends to balance out the parasitic loss of neutrons from Doppler "broadening", so the Doppler (fuel temperature) coefficient decreases significantly.

The impact of including minor actinides in an oxide fuel core was studied in Reference 14, with a key figure reproduced here as Figure 7. It is quite evident that adding even a relatively small amount of minor actinides can have an impact on the reactivity feedbacks for the oxide fuel, and almost certainly for other fuel types as well. If one adds $10 \%$ minor actinides to U-Pu fuel, a $40 \%$ increase in sodium void worth and a $55 \%$ decrease in Doppler feedback may result.

The metal fuel reprocessing currently envisioned does not include separation of the minor 


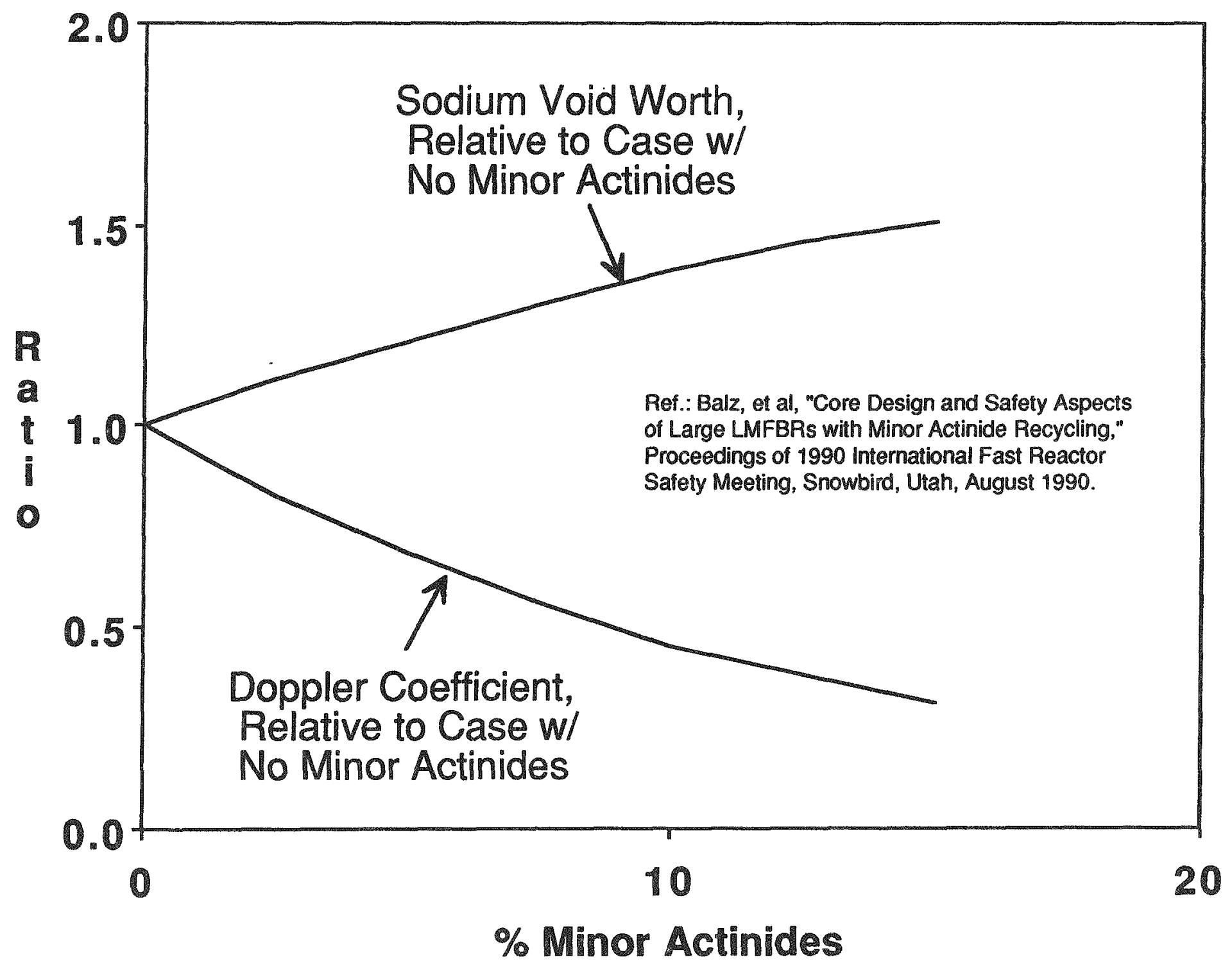

Figure 7 Influence of Minor Actinide Content on Sodium Void and Doppler Reactivity Feedbacks (Oxide Fuel). 
actinides, so they will be recycled into subsequent batches of wel. We are nut sure what fraction of minor actinides would eventually reside in an equilibrim core, alhough if should be below that of the LWR spent fuel. (Therefore, the fraction of minor actinides should be highest for presh fuel from LWR recycles). ANL probably has a more accurate estimate, although the uncertainties involved in the cross sections, and therefore the burnup calculations, must be fairly large.

Clearly there is some significant safety performance penalty involved in recycling the minor actinides in PRISM, but one might be able to compensate for the changes in the reactivity feedbacks. This is particularly true if the minor actinide inventory is only 2 or $3 \%$, which seens likely if the current fuel processing strategy is implemented. Another concern is its effect on thermal conductivity, fuel clad chemical interactions, and eutectic formations at the clad/fuel interface. For the time being we simply note that the minor actinides could be a significant factor in the PRISM Passive reactor shutdown fearre, especially if the minor actinide loading exceeds a few per cent.

Finally, DOE, ANL, and GE have recently focused efforts on some of the "actinide burning" characteristics of the metal fuel cycle. It has been pointed out that the minor actinides from LWR spent fuel. which are significant burdens for the plamed repository (Yucca Mountain) due to their long radioactive half lives and their high toxicities, could be consumed as tuel in the integral fast reactor. Should this option be further pursued, it is likely that the minor actinide inventory in future metal-fuel ALMRs (PRISM?) could be much higher than 10\%. A machine built primarily for consuming minor actinides would likely have a very large positive sodium void worth and a very small Doppler feedback, judging by the trends shown in Figure 7.

\section{Key Issues/Concerns in Section 4.5}

- While sodium boiling events are highly improbable, the possibility exists for a potentially serious accident. The key issue is whether the energetics from such a worst case scenario could be contained within the vessels and containment dome. This appears to be true, but we need additional data before we can properly estimate the energy release during such an excursion.

- There are some conflicting design objectives with respect to the sodium void worth. First, the use of metal fuel appears to reduce the likelihood of sodium boiling (due to the passive shutdown), but it also increases the sodium void worth. Second, the consumption of minor actinides makes for a cleaner ALMR fuel cycle but their addition to an ALMR core tends to increase the sodium void worth and reduce the Doppler coefficient. Their effect on fuel characteristics (i.e., thermal conductivity, $\mathrm{FCCI}, \ldots$...) are also unknown.

\subsection{Flow Blockage}

This is an issue that was originally raised by the NRC staff, and we defer to them regarding the GE response in Appendix G. A few comments are added here just to note our perspective on this postullated event.

The event at Fermi in 1966 involved a piece of zirconium liner that had broken loose and moved into the core inlet region, creating a partial flow blockage that caused fuel damage and might have lead Io a much more serious event. As a result, the PRISM core inlet region is designed to prevent such a blockage. About the only means of developing a comparable blockage would require a piece of flexible material, such as aluminum foil, and would require a complete wrapping of that material 360 degrees around an assembly inlet. It is difficult to consider such a development as being even remotely possible. 
The concern raised by the NRC staff regarding PRISM is a manufacturing defect which might leave an assembly without the slots which are to allow the sodium to pass into the assembly. While unlikely, this certainly seems to be a possibility. The key point in the applicant's response is that the defect would be detected before the reactor was taken to full power, and that at low power any reactivity addition resulting from sodium voiding or fuel slumping could be accommodated without a major accident resulting. This response seems reasonable, assuming the applicant assures that the proper instrumentation and procedures are implemented, but it also seems to indicate a shift in policy. In the past, the applicant has chosen to de-emphasize the role of the operator in assuring the safety of PRISM, but in this case they are indicating a reliance on a person and/or a safety system detecting a problem and preventing a problem from becoming much more serious.

Key Issues/Concerns in Section 4.6

- The NRC staff's concern about manufacturing defects seems to have merit, and something should be added to the operational procedures to identify such a defect before going to power.

\subsection{Electromagnetic Pumps}

The EM pumps are important to the safety of the PRISM reactor, particularly if there is a failure to siram. The fast that EM pumps have no inherent coastdown (almost no inertia) and must rely upon synchronous machines operating in parallel to provide a coastdown increased our concerns further. However, the addition of the GEMs helps to relieve some of these concerns, as a significant loss in pumping should lead to a significant reactivity decrease as the sodium level in the GEMs drops. The EM pumps remain important to the safety of PRISM, but the assurance of the coastdown may not be as crucial as it was prior to the addition of the GEMs (see also section 4.16.3.3.4).

\subsubsection{EM Pump Characteristics}

In order to fully analyze the transient behavior of the PRISM reactor coolant system we needed far more detailed information on the pumps and the synchronous machines than the applicant had provided in the PSID or in the open literature. After some discussions, the applicant provided us with the design details they were using for their own modeling. As this data was in the form of computer code input. some effort was required to interpret the information and to make it fit into one comprehensive model. Eventually we developu w of of equations and curves that resembled those provided by the applicant.

A key pump performance "head" curve was provided by the applicant as Figure G.4.7-2 in the new appendix of the PSID. Our corresponding head curve is included here as Figure 8 . The two figures are very similar, except that the applicant has chose to plot the head curves for different voltages, at the reference speed of 600 RPM, and we plotted the head curves at different speeds (synchronous machine) for the reference voltage of 627 volts. Presumably, this curve was drawn from a designer's perspective, illustrating their options for driving the pumps at higher voltage. However, the pump performance at different speeds are more important to transient analysis, particularly when the synchronous machines are coasting down.

One feature indicted by Figure 8 and the applicant's Figure G.4.7-2 is that, near the operating point of $11500 \mathrm{gpm}$ and $115 \mathrm{PSI}$, the slop of the head curve is extremely steep. This means that a slight increase in flow will cause a sharp reduction in head, which in turn will reduce the flow. Alternately, a slight drop in flow will lead to a large increase in head, which will increase the flow. Thus, these pumps will have a strong tendency to run at nearly constant flow, because any change in flow will trigger 


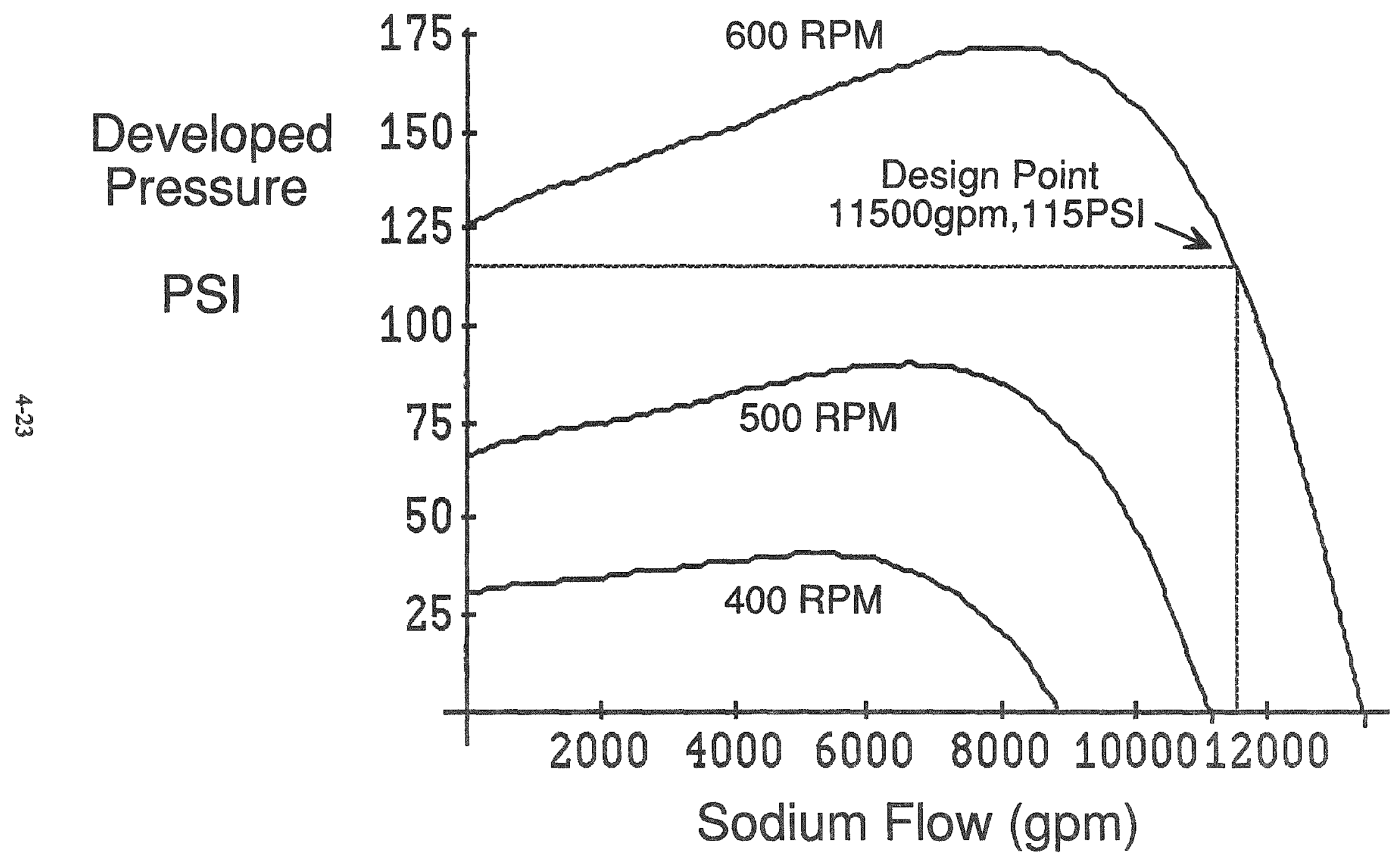

Figure 8. PRISM EM Pump Head vs. Sodium Flow Rate. 
a significant change in driving head, pushing the flow back toward the nominal value.

Our analysis indicates that the applicant is using a credible set of pump performance curves, which include not only the head curves, but also efficiency curves and torque curves. These pumps are more complex than corresponding centrifugal pumps.

\subsubsection{Pump Control}

The applicant states that the Power Conditioning Unit (PCU) voltage and frequency are controlled by the primary controller. As shown in both Figure 8 and Figure G.4.7-2, changes in either voltage or frequency can have a strong impact on the driving head provided by the EM pumps. Therefore, the controller should be able to impact substantially on the sodium flow rate to the reactor. This is an advantage for the control system engineer, but it establishes a strong link between the non-safety grade plant control system and the reactor cooling capability, and thus opens some safety issues. Again, the addition of the GEMs may be an important factor in determining whether or not this is acceptable.

\subsubsection{Synchronous Machines Isolated}

The movement of the synchronous machines into a region that is seismically isolated appears to be a clear improvement in the design. The potential vulnerability of the cables connecting the synchronous machines to the EM pumps, as they crossed from a non-seismically isolated area to the seismically isolated area, was a major concern before the design revision, which now places them both (i.e., synchronous machines and EM pumps) in a seismically isolated area.

\subsubsection{Tuning the Coastdown}

One advantage of providing an artificial pump coastdown using the synchronous machines is that one can adjust the coastdown. The applicant is planning to take advantage of this option by dropping the voltage quickly initially, in response to an expected quick reduction in power, and saving some of the energy for an extended coastdown later in the event. While not expressly stated by the applicant, they obviously have the option of using bigger/heavier synchronous machines if they need more coastdown energy.

In principle, there seems to be no problem with the applicant's utilization of this option. However, there are limits as to how much flexibility the operator, or the control system, should have over the flow coastdown, so this is one area to watch as the PRISM design undergoes further design revisions.

The flow coastdown calculated by the applicant is reported to be represented in Figure G.4.7-1 of Ref. 1. Our independent calculations indicated a lower flow rate, so we requested further information from the applicant. They provided the tabular printout showing the sodium flow rate through the reactor and through the pump during a nominal flow coastdown event. Oddly enough, the tabular printout showed a flow coastdown that certainly appears to be faster than that shown in Figure G.4.7-1. The applicant believes that some distortion occurred during the preparation, reproduction, or printing of Figure G.4.7-1. Regardless, the trend in the tabular data provided by the applicant were relatively close to our analysis, as shown in Figure 9. Our calculation shows a somewhat quicker coastdown for the first few seconds. It is also noted that the flow differential between the pumps and reactor in the ARIES calculations seems too high (they should be almost identical). While these discrepancies are a modeling concern, they are not to be considered a safety concern at this time, partly because the GEMs provide a strong negative reactivity feedback as the pumping falls off. 


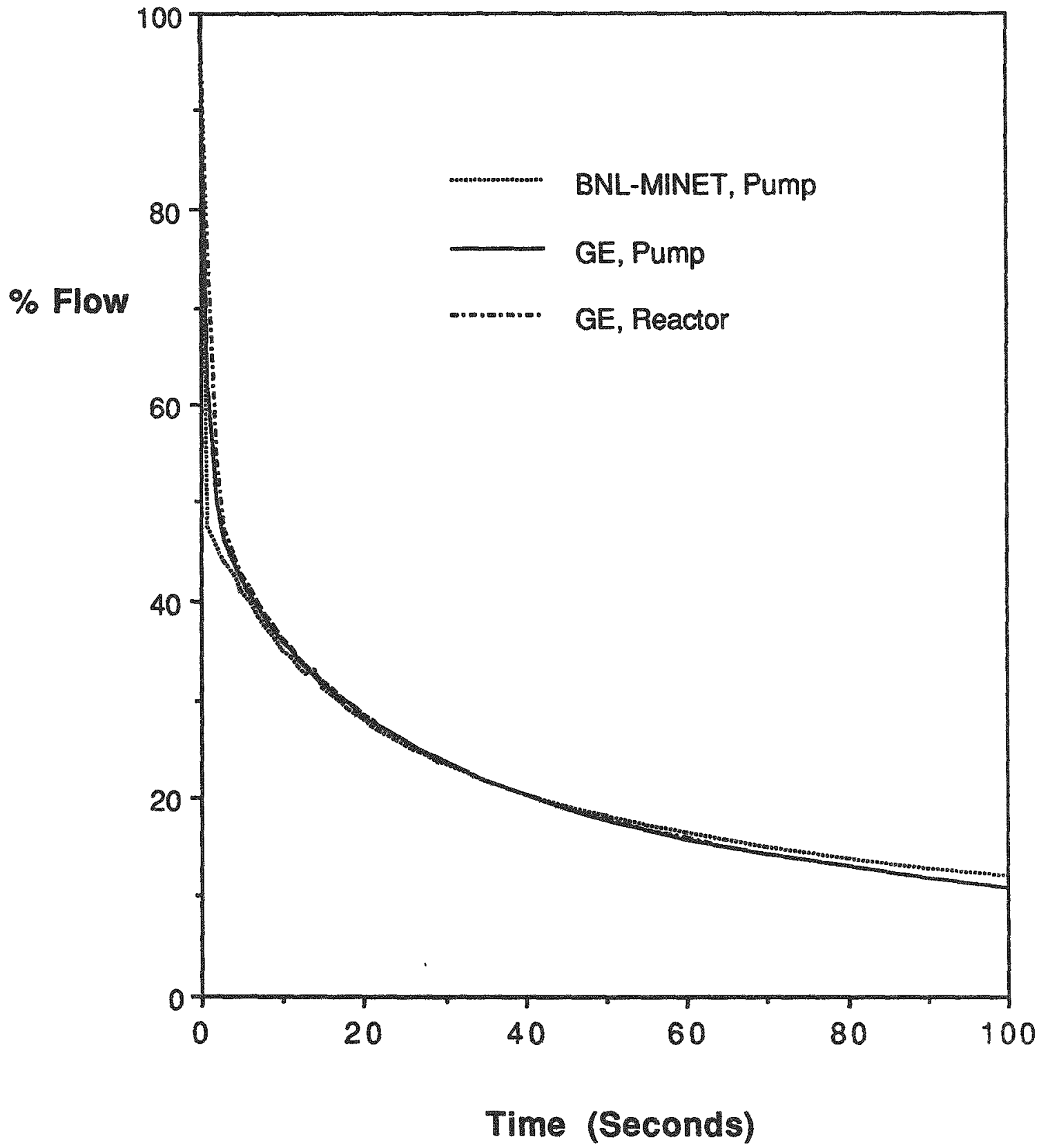

Figure 9. Predicted Flow Coastdown after a Trip from ARIES (G.E.) and MINET (BNL). 
While a couple of questions about the flow coastdown remain unanswered, it must be remembered that the applicant has considerable control over the pump coastdown. If the flow coastdown provided by their current design is not high enough or long enough, they need only modify the synchronous machines to get the coastdown they need. And again, the addition of the GEMs to the design reduces a requirement for a high initial flow during the coastdown.

\subsubsection{Rods in Before Pump Trip}

Because of the potentially serious consequences of the unscrammed loss of flow (ULOF) events, even given that the passive reactivity shutdown is likely to work, it is not prudent to trip the EM pumps without verification that the scram has taken place. The applicant had not considered this previously, but has indicated their intent to remedy this oversight. In particular, they now indicate they will verify that the neutron flux in the reactor is, in fact, decreasing before tripping the pumps.

\subsubsection{The 3 of 4 Pump Coastdown Case (See also Section 4.16.3.3.4)}

Prior to the addition of the GEMs, the safety margins (to sodium boiling) for the ULOF were modest, and very small if one of the four synchronous machines failed to provide a coastdown. The applicant's analysis indicated that the other three synchronous machines would coast down more slowly if one failed completely, as the flow resistance in the piping would be less, thereby reducing the drag on the remaining pumps/machines. We have only recently been able to analyze this event, as discussed in Section 4.16.3.3.4

\subsubsection{EM Pumps at High Temperatures}

The applicant describes a Class 1E thermal shutoff system (TSS) that backs up the reactor protection system (RPS) and trips the pumps when the cold pool reaches $811 \mathrm{~K}\left(1000^{\circ} \mathrm{F}\right)$. This is done partly to ensure that they will have sufficient "electrical integrity" to coastdown.

A further consideration is the Curie point temperature, above which the magnets stop behaving like magnets, i.e., stopping driving the sodium magnetically. The applicant doesn't explicitly state this temperature, which is dependent on the materials composition and can therefore be adjusted (within limits). They do state that the Curie point will be higher than $811 \mathrm{~K}\left(1000^{\circ} \mathrm{F}\right)$, so both the RPS and the TSS should act to preclude the temperature getting so high. In addition, the Curie "Point" is really an interval of rapidly changing magnetism, so the Curie Point failure would be at least somewhat gradual providing some coastdown.

\subsubsection{The EM Pumps - Summary}

Because the time provided by the pump coastdown is vital to the inherent shutdown response, the pump inertia plays a crucial safety role. In order to obtain the necessary coastdown with EM pumps, the applicant must utilize the synchronous machines. However, EM pumps apparently provide some real advantages in the operation of PRISM, at least in the opinion of the applicant. The addition of GEMS to the reactor system design has reduced our concerns significantly for the instantaneous loss of a pump, as a loss in pumping will result in a quick reduction in reactivity and reactor power. 


\section{Key Issues/Concerns in Section 4.7}

- The pump coastdown reliability is a crucial element in the PRISM Passive Shutdown during ULOF events. The designers choice to supply the coastdown using the synchronous machines has resulted in potential failure modes that would not be present if centrifugal pumps were used.

\subsection{Sodium/Water Reaction Pressure Relief System (SWRPRS)}

Because the applicant has substituted a helical-coil single-wall tube steam generator for an older straight double-wall tube model, much of the story regarding possible sodium-water reactions has changed significantly from the previous design. The newer single-wall tubes are thought to be more likely to fail, but there is a design feature (central conduit) in the new unit that should help to mitigate such an event.

The major objective is to protect the IHX, which forms a boundary of both the primary coolant system and the containment system. The secondary sodium passes through the IHX tubes, and the applicant claims that a 1000 psi pressure pulse (generated in the steam system from the tube failure) could be absorbed safely. The objectives in protecting the $\mathrm{IHX}$ are to prevent a larger pressure pulse and to prevent the sodium-water interface from passing into the $\mathbf{I H X}$, where the ongoing chemical reaction could damage the $1 \mathrm{HX}$ tubes.

The IHTS isolation valves form one line of defense, as their closure would protect the IHX from both pressure pulses and the sodium-water interface. Of course, these valves are active components, so there is a limit regarding the level of reliability that can be assured.

The more reliable (and far more complex) defense comes from the passive rupture disks. Because of the rupture disks, the dump tanks, and some steam flow limiters, a large scale sodium-water reaction can be accommodated without a major pressure build-up. The challenge is to assure that the sodiumwater interface can not reach the IHX. As the reactions are developing in the steam generator, the pressure builds, and pushes the sodium level up into the argon cover gas in the top of the steam generator. Once the sodium level gets high enough, the sodium spills into a central conduit, which bypasses the helical coil tubes and lets out near the rupture disks. Because of this bypass feature, the sodium in the steam generator can be driven through the rupture disks without the argon cover gas pressure getting too high. Since any sodium in the lower portion of the steam generator and most of the sodium between the pump and the bottom of the steam generator will exit through the rupture disks, it is only the sodium-water mixture in the top of the steam generator that is a concern with respect to possibly passing into the IHX. Some of the sodium-water interface could push upward from the argon cover gas and back into the piping coming from the IHX (the hot leg). This would involve pushing sodium upward from the IHX to the IHTS pump, from which it would flow downward toward the rupture disks. Whether this could happen depends partly on the pressure differentials in the IHX and the IHTS. It seems fairly clear that one could design the plumbing to prevent this possibility, as long as they can keep the pressure drop though the steam generator to reasonably low levels (which the conduit appears to accomplish).

Therefore, there appear to be two fairly reliable means to prevent a large sodium-water reaction from compromising the $1 \mathrm{HX}$ tubes. However, there is one factor that looks to be at odds with a normal safety approach, that being the use of sequential (redundant) rupture disks (both must function for the drain process to begin). From a safety viewpoint, two parallel rupture disks would increase the likelihood of achieving a timely dump of the sodium. However, rupture disks are passive devices and they apparently tend to open at lower pressures rather than to remain open at too high a pressure. Therefore the applicant is using sequentially redundant rupture disks to assure they don't open by mistake and expel 
the IHTS sodium. Still, the proper opening of those rupture disks is very important in responding to a large sodium-water reaction, so we would recommend some parallel redundancy in the design as well.

\section{Key Issues/Concerns in Section 4.8}

- The protection against the results of sodium-water reactions appears to be largely satisfactory, although some closer examination is advisable as the design matures. The designer's choice to use sequentially redundant rupture disks but not parallel-redundant rupture disks helps to prevent the inadvertent dumping of the intermediate loop sodium, but doesn't help in assuring the disks will blow open if a large reaction takes place.

\subsection{Reactor Vessel Auxiliary Cooling System (RVACS)}

Previous analysis of the RVACS system, as described in Ref. 1, indicated the system would likely perform even better than the applicant was assuming. However, there were concerns about a couple of issues, and these concerns were brought up in the SER [Ref. 2], and the applicant responded to these in Appendix G. One of these issues was a postulated event in which RVACS was assumed to be fully plugged for 36 hours, leading to an adiabatic heat-up that lead to significant damage before the end of 36 hours. The other issue was the possibility of cumulative damage to the vessel and structures if there were to be repeated cases where RVACS was the only heat removal system available. Both of these issues were raised at a time when the applicant was taking the position that the operator had little or no safety function and that RVACS was so failure-resistant that other decay heat removal systems were comparatively insignificant and that there was no reason to address accident mitigation. Since the applicant has altered its position with respect to the operators and accident mitigation, adding a containment dome among other things, the importance of these issues has diminished somewhat. In particular, the bounding event 3 has been modified from a 36 hour adiabatic heat-up (see Section 4.16), and we are more inclined to give credit for the ability of the normal heat removal system and the ACS to perform well under natural circulation conditions, which reduces the likelihood that the RVACS would be the sole means of decay heat removal to perhaps once or twice during the plant's lifetime. Further, should there be cumulative damage to the vessel or structures due to an over-reliance on RVACS or an under-performance of RVACS, it seems more likely to be an investment protection issue than a pressing safety issue, i.e., if the vessels suffer significant damage the reactor would have to be shut down for a detailed inspection, and subsequent hearings, before operations could resume.

However, when the applicant chose to increase the power produced by a PRISM reactor module from $425 \mathrm{MWt}$ to $471 \mathrm{MWt}$, they did so without modifying the RVACS. As a result of this and other changes, the amount of decay heat to be removed through RVACS increased by around $20 \%$ (note: there have been a couple of previous decay heat curves; the $20 \%$ increase is relative to the curve we had been using in prior analyses). Therefore, we had to repeat our evaluation of the RVACS performance, and this is discussed in the remainder of this section, and to some degree in Section 4.16, as well.

\subsubsection{Analytical Tools Used for RVACS Calculations}

The applicant's analyses of the PRISM LOHS events are performed using special-purpose computer codes that represent the system configuration in some detail. The advantage is that unique features of the design can be easily programmed into such special-purpose models. The disadvantage is that the validation base for such a code is very limited, as the code is largely wired for a system that exists only on paper. 
The BNL analysus uilize the MINET Code [Ref. 19 and 20], which is a generis fluid flow and heat ironsfer metwork transient analysis package, which has been used to analyze various sodium, water, and ga-cooled systems. The advantages of using MNET are that the validation base is sound and Uiverse, and that foirly sophisticated fluid dynamics and heat transfer models are available. The principal disadvanage is that features unique to PRISM can be difficult to factor into the calculation.

By cross-comparing results from the applicant's calculations and those generated at BNL using MINET, wean get a reliable prediction of the likely RVACS performance. There is little in common beiween the models, and the chances of replicating errors are viewed as minimal.

\subsubsection{PRISM System Conditions During RVACS Operation}

Under normal operating conditions. i.e. with the EM pumps performing normally, the sodium level hetween the liner and the reactor vessel is quite low. Once the postulated event begins and the EM pumps are iripped and coasted down, the system pressure differentials decrease, and the sodium level between the liner and the reactor vessel rises to almost the sodiun level in the hot pool. Due to decay hear levels that exced the RVACS heat removal capability, after about 4 hours of heat-up the system sodium expands enough for the hot pool sodium to spill over the liner and low downward along the inside of the reastor vessel. System conditions during this time frame are illustrated schematically in Figure 10.

Once system conditions are as shown in Figure 10, a long term heat-up and eventually a cool-down process begins. Once the hot pool sodium starts flowing down along the reactor vessel wall, the RVACS performance is enhanced significantly. However, temperatures will continue to increase for several hours until the decay heat decreases and the RVACS performance increases enough for the temperatures to level off. Only after several more hours would the decay heat level fall off enough for system temperatures to decrease.

\subsubsection{Analyses of RVACS Performance During Reference LOHS}

The predictions of the reactor outlet sodium temperatures during the reference RVACS event, in which the decay heat is removed solely through RVACS, are shown in Figure 11 . It is noted that the peak sodium temperatures develop after 24 hours into the event, and are some $40+$ degraes $K$ below the ASME C limits for the reactor vessel. Both calculations are in excellent agreenent regarding the peak temperature. There is also agreement as to when the sodium will expand enough for the spill-over to develop, about 4 hours into the transient. The difference in reactor outlet sodium temperatures at that time is believed to be due to a feature in the applicant's model that computes the heat transfer from the hot pool sodium, through the liner, to the sodium that remains stationary between the liner and the reactor vessel. Because there is no equivalent in the MNET model, the hot pool sodium remains a little hotter, and this propagates through the primary loop and results in reactor temperatures that are a little too high. However, once the spill-over begins, this difference disappears, and the calculations match up well after 8 or 9 hours into the event. Any differences in temperatures thereafter are not considered to be significant.

In addition to the calculations represented in Figure 11, we have performed several other calculations using MINET, the PASCOL code, or using simpler models. These calculations have consistently shown that RVACS will perform at least as well as the applicant claims, and that the system has a high degree of fault tolerance. 


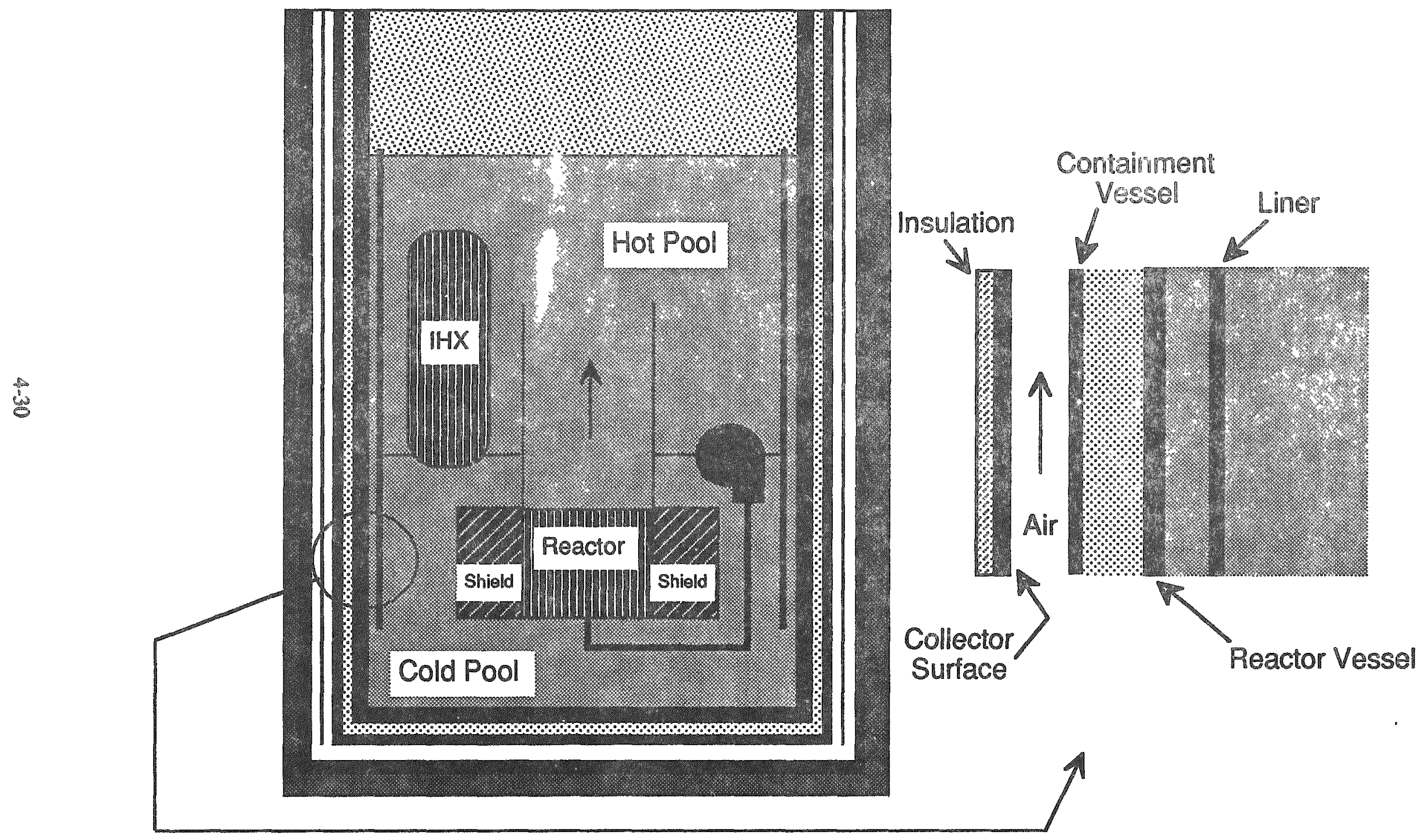

Figure 10. PRISM During RVACS Cooling Event (No Vessel Leak). 


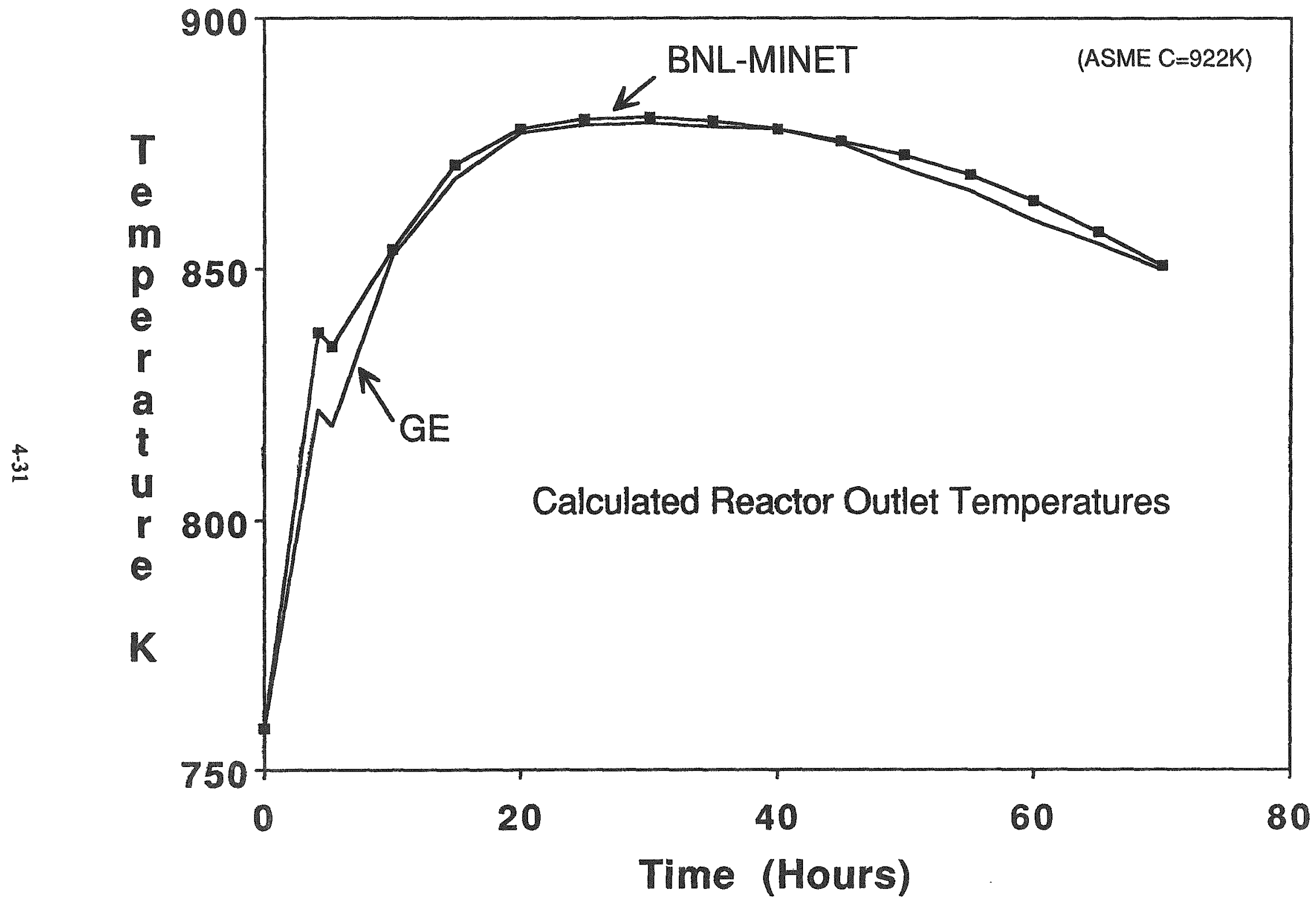

Figure 11. PRISM LOHS with RVACS Performing Normally. 


\subsubsection{Analyses of Bounding Event 3A}

This event, which is also discussed in Section 4.16 , involves a postulated $75 \%$ blockage of the air flow ducting which results in a reduction in air flow along the containment vessel. The corresponding LOHS event results in higher sodium temperatures, although the increase appears to be acceptable. The reactor outlet sodium temperatures from the applicant's calculation and from BNL's MINET analysis are shown in Figure 12. There is very strong agreement between the two simulations, although MINET places the peak temperatures after 40 hours a few degrees higher than the applicant's prediction. In either case, the predictions indicate that the reactor outlet temperatures may exceed ASME level " $\mathrm{C}$ " limits for the vessel, which is primarily an investment protection issue. However, the applicant may believe that a $75 \%$ blockages of these very large air flow ducts is unlikely, and further, that failure to clear a pathway within 36 hours is hard to imagine. Regardless, it appears that a postulated LOHS, involving failure of the normal cooling system, the Auxiliary Cooling System (ACS), and a 75\% blockage of the air flow ducting can be tolerated without severe damage to the reactor and/or coolant system.

\subsubsection{Analyses of Bounding Event 3B}

This event, also discussed in Section 4.16, involves a postulated full blockage of the air flow ducting for the first 12 hours and a $25 \%$ opening thereafter. The corresponding LOHS is truly adiabatic for the first twelve hours, and would cause significant damage. The reactor outlet sodium temperatures predicted by the applicant and BNL are shown in Figure 13, and are again in excellent agreement with each other. Both calculations place the peak temperatures in the range of ASME level D limits for the vessel. These temperatures are clearly high enough to endanger the plant from an investment standpoint, although it does not appear likely to lead to significant fuel failures or radioactive releases. This is quite impressive, given that total failure of 1) the normal cooling system, 2) the first backup cooling system, and 3) a nearly failure-proof emergency cooling system for 12 hours, is a series of failures that is nearly inconceivable, barring a very thorough act of sabotage.

\subsubsection{Analyses of Vessel Leak Case}

The design basis for the PRISM containment vessel is a leak in the reactor vessel. In such an event, the containment vessel is to catch the leaked sodium and prevent the sodium level within the reactor vessel from dropping below the IHX inlet. Maintaining the sodium level is important in order to preserve a pathway from the hot pool to the cold pool. With the lower sodium level, the sodium can not spill over the liner (at acceptable temperatures, anyway), so thermal conduction across the liner, the sodium trapped between the liner and the reactor vessel, the reactor vessel, the sodium between the reactor and containment vessels, and across the containment vessel, must be adequate in order to prevent damage. This situation is illustrated in Figure 14. Note that there is a trade-off here, in that the improved heat transfer between the two vessels (conduction heat transfer is more effective than radiation heat transfer) compensates to some degree for the loss of the sodium spillover and direct heating of the inside of the reactor vessel.

In the original PSID [Ref. 1], the applicant analyzed this event and predicted lower reactor temperatures than for the reference RVACS event, i.e., with the sodium spill-over functioning normally. They did not repeat the calculation at the higher decay heat levels, and assume that again the reactor temperatures would be lower than for the reference RVACS case discussed above.

We performed two calculations for this event, with the results as indicated in Figure 15. Both calculations utilize the same estimate for the rate of heat transfer via conduction for various sodium temperatures, and thus are not truly independent. Also, there are several key parameters that contribute 


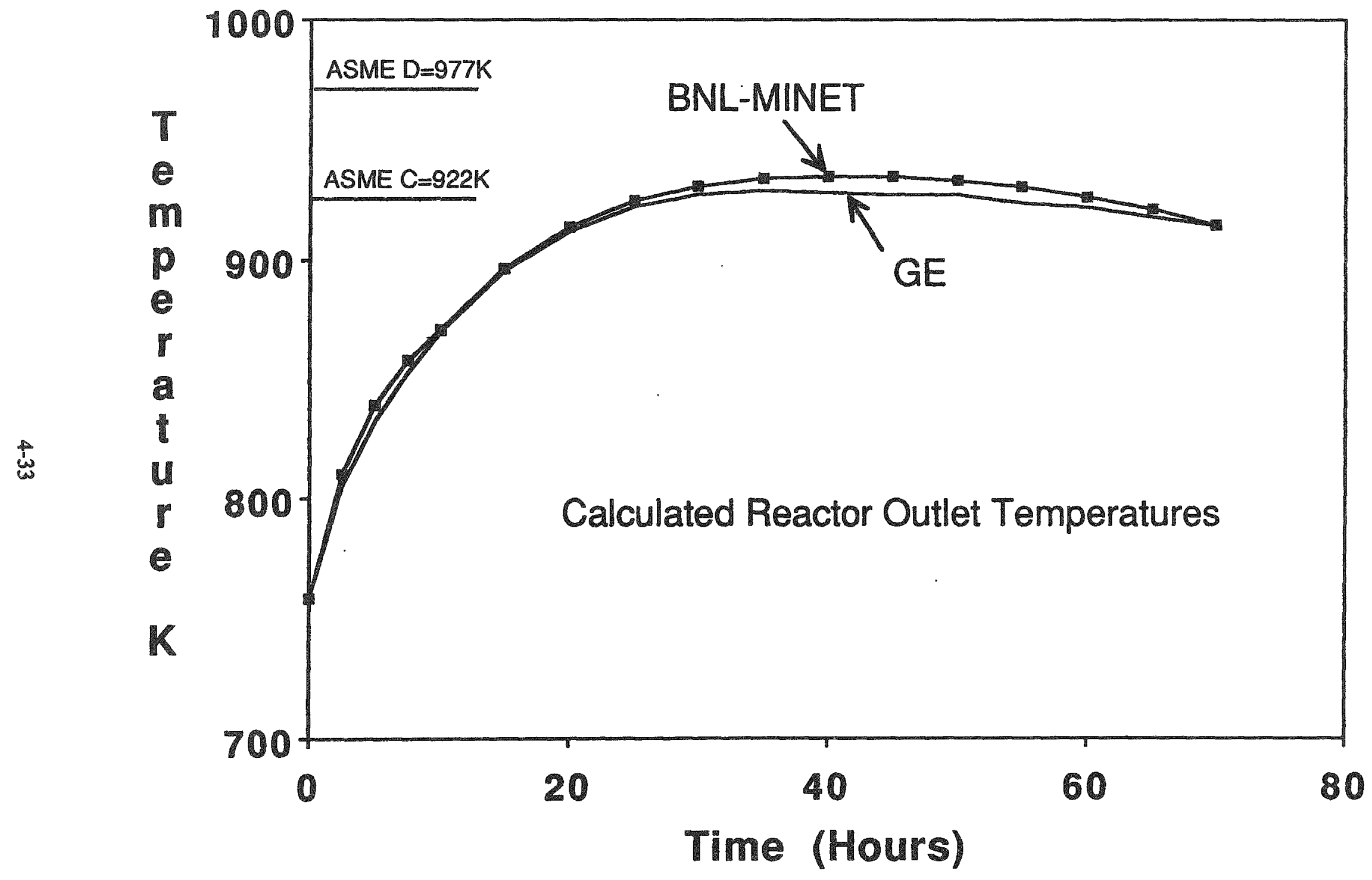

Figure 12. PRISM LOHS with RVACS Air Flow Ducting $75 \%$ Blocked. 


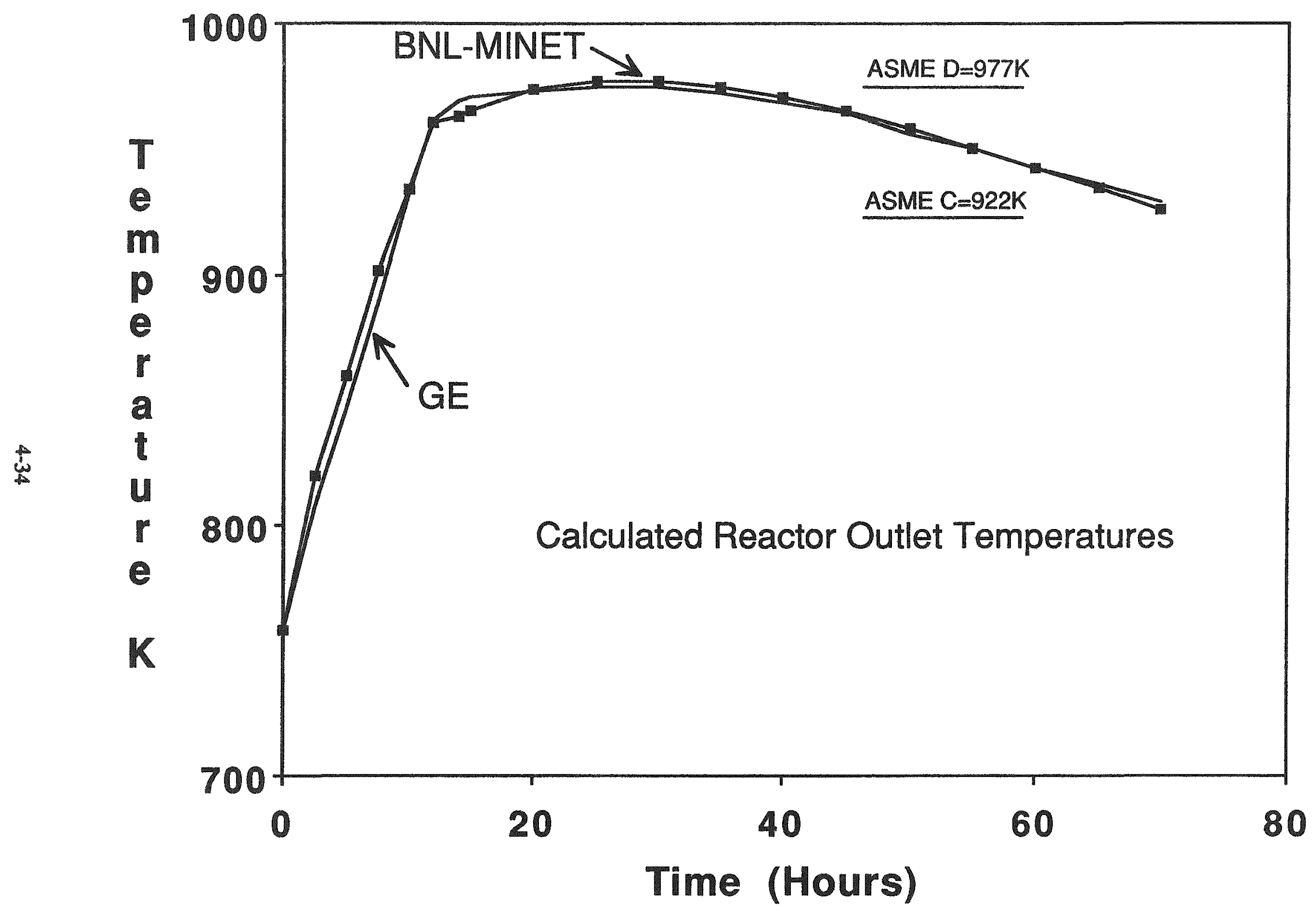

Figure 13. PRISM LOHS with RVACS Blocked 12 Hours; Open 25\% Thereafter. 


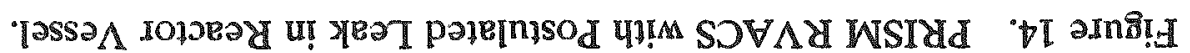

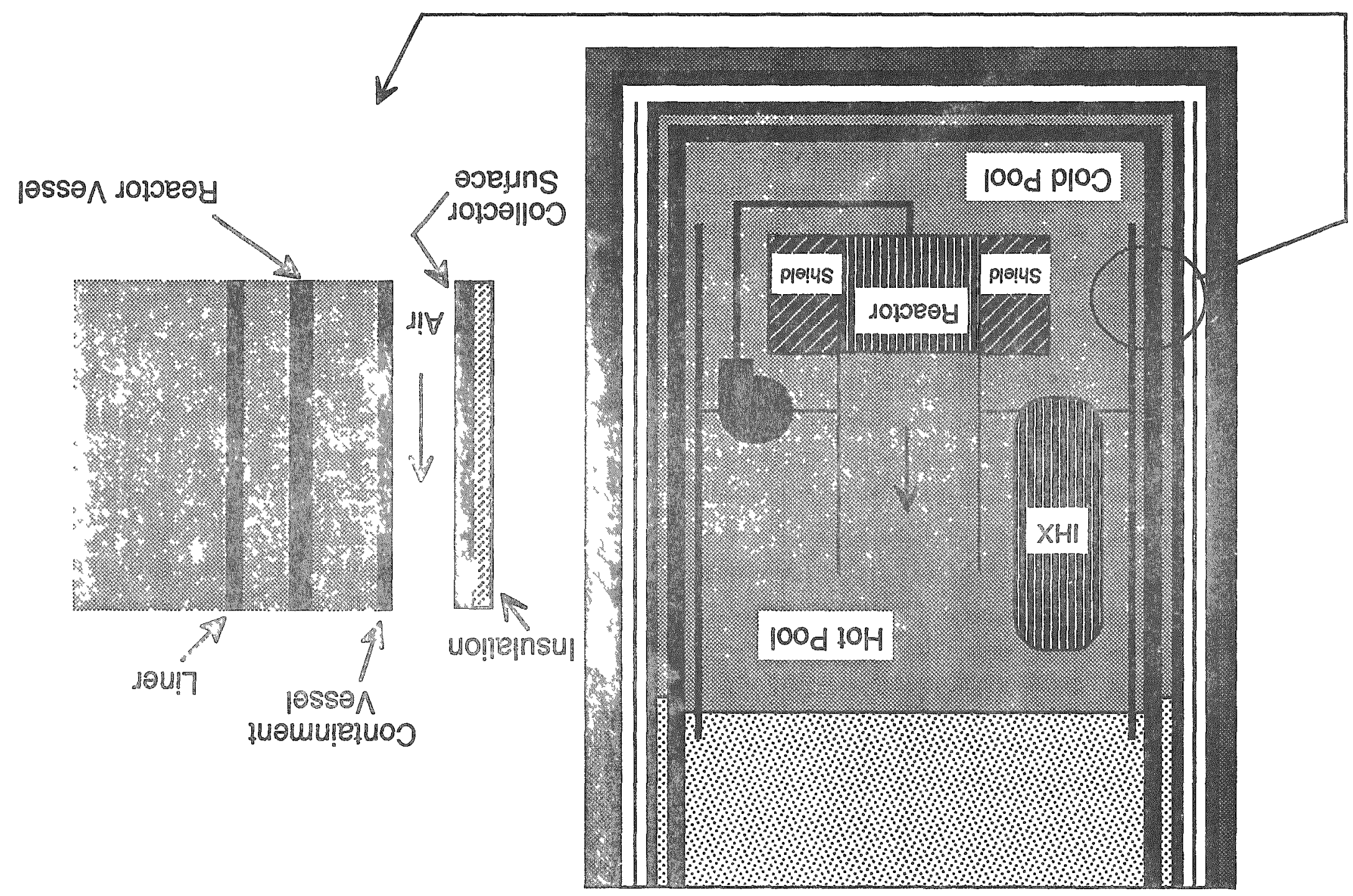




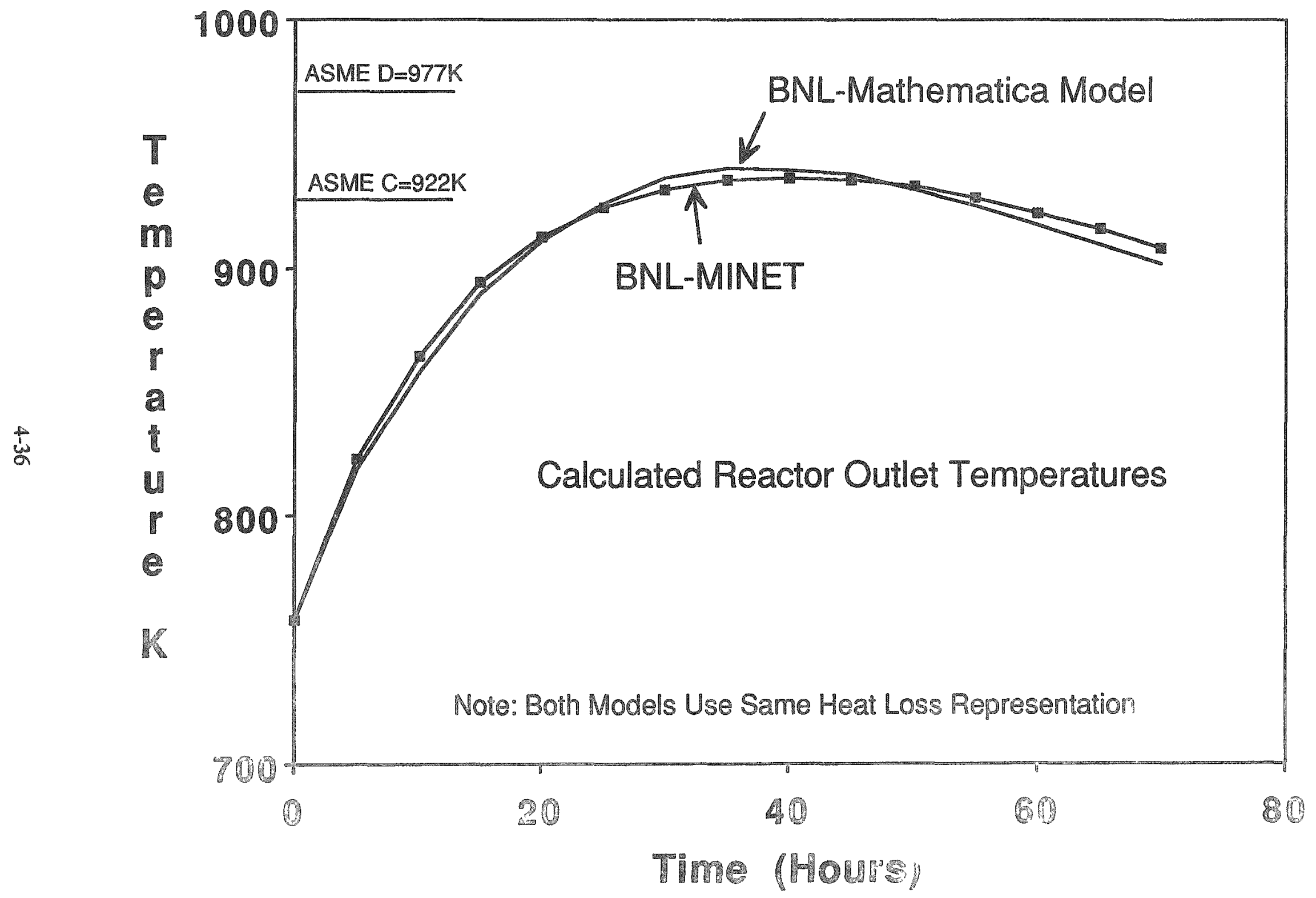

Figure 15. BRISM LOHS win Leak in Pegeror Vessel (No Spill-Oner). 
to the predicted heat loss, and some of these parameters may not be identical to those used in the results described in previous sections. Given those qualifications, we can see that the predicted temperatures exceed ASME level C limits after about 36 hours, before dropping off gradually thereafter. Once again, these temperatures are in a range where the investment may be jeopardized, but fuel damage or radioactive releases would be unlikely.

\subsubsection{PRA Implications}

Almost from the outset we have struggled to predict a credible RVACS failure probability for usage in a PRISM Probabilistic Risk Assessment (PRA). It simply makes little sense to postulate failure probabilities when the only apparent failure modes involve major seismic events or sabotage. Only recently has an alternate failure mode become apparent, and that is largely due to the increased decay heat load and higher normal operating temperatures which have reduced the safety margins. As mentioned before, there are several parameters that determine how well the RVACS will perform, including heat transfer coefficients, emissivities, thermal conduction in the structures, and various flow resistances. Naturally, there is a degree of variability in each of these parameters, which could be represented using some distribution functions. The likelihood of some given level of performance would depend on the distribution functions for several of these key parameters. If several parameters are in the least favorable portions of their distribution functions, the RVACS performance could conceivably drop enough to cause problems. (However, the applicant's analyses of several variant cases do suggest a high degree of robustness, in that poor performance in one area can be compensated by other parameters).

If one examines the RVACS performance for the reference event, the safety margins and fault tolerance is high enough to cover a lot of pessimistic assumptions. However, if one examines a variant case, such as a postulated vessel leak, then there is some chance of an off-performance leading to damage. For example, if there is a $1 \%$ chance of fuel damage in the event of a reactor vessel leak, and a . $01 \%$ chance of a containment vessel failure given a reactor vessel leak, then it is the $1 \%$ possibility of RVACS not performing well enough that may be the greater concern.

This alternate means of representing the performance of such a passive systems in a PRA is still being developed, and is not reflected in this report. However, we believe it could be an important step toward properly representing the type of passive systems that are now being developed for use in advanced reactor concepts.

\section{Key Issues/Concerns in Section 4.9}

- The RVACS may be the greatest strength of the PRISM design, and it is very difficult to defeat. Our present belief is that possible degradation (with time) may be the most credible "failure" mode. While high temperatures have been predicted for some very unlikely events, they have shown to have been investment concerns since ASME service limits were passed. However, the vessel integrity was not severely challenged.

\subsection{Control Room}

This is an issue that was originally raised by the NRC staff, and we defer to them regarding the applicant's response in Appendix G. We add a few comments here just to note our perspective on the control room.

While there are many characteristics of the PRISM design which will reduce the role of the operator, there are still a few important functions to be performed. The passive (inherent) shutdown 
reduces the importance of scramming the PRISM reactor immediately. However, if failure to stram results in sodium boiling (unlikely, but possible), it is essential to scram the reactor quickly. There are other operator functions involving plant startup (to check for blocked fuel assemblies), repositioning of the control rod stops, and responding to seismic events that are important to safery, and the operator's ability to quickly assess the situation and to take corrective action must be assured.

\section{Key Issues/Concerns in Section 4.10}

- We believe the operators must have the ability to scram the reactor within a few seconds of detecting a major problem.

\subsection{Emergency Preparedness}

The applicant has taken the position that the likelihood and possible consequences of accidents are such that formal off-site emergency planning involving early notification, detailed evacuation planning, and provisions for exercising of the plan is not required. Previously, their arguments were based mostly on the low likelihood of a serious accident. More recently, GE has placed increased emphasis on accident mitigation.

While there are some very clear safety advantages in the PRISM concept, it is very difficult to develop credible probabilistic numbers at this stage. The safety of PRISM is based largely on the passive reactor shutdown mechanism and the passive decay heat removal system. Because the PRISM ternary fuel is still under development (very few pins with 26\% Pu have been tested, and those have run only to low burnup levels), there is still a great deal of uncertainty involved in the passive shutdown. Also, the large positive sodium void reactivity worth could pose a severe problem should the passive shutdown fail to prevent sodium boiling. The passive decay heat removal is fundamentally much simpler and has held up well to close scrutiny. However, there is little experience with such systems, and the PRISM safety rationale depends very heavily on the performance of this system.

With respect to accident mitigation, the applicant must deal with a different problen. Over the last few decades, ANL has made considerable progress with the metal fuel, but has not developed the type of extensive data base required to analyze severe accidents. In particular, there is little data available on the behavior of fission products in molten metal fuel, particularly regarding how it behaves in sodium pools. Also, the key mechanism required for terminating an HCDA, if one should ever develop, is the fuel extrusion. Recently, ANL has been preparing to develop the required data base, and has been providing some best guesses as to what the data is likely to look like. They make sound arguments as to the likelihood of key fission products remaining in either the molten fuel or the sodium pool and as to the likely speed with which the fuel extrusion would take place during a power excursion. However, there is simply little or no data available at this time to justify their arguments.

The applicant, as well as ANL and DOE, has presented the NRC with arguments about not needing detailed emergency evacuation planning, based on the information available at this time. This may be difficult judgement to make at this time, and it may have to be deferred until some of the "severe accident" data becomes available.

Key Issues/Concerns in Section 4.11

- Most accidents postulated for PRISM would be slow, and the designer's arguments with respect to using ad-hoc emergency planning have merit. However, those very low probability HCDA events could be very rapid. If the data demonstrates that a HCDA is highly improbable, and if 
it appears that such an event can be contained in the vessels and the dome, then an ad hoc emergency response may be appropriate.

\subsection{Role of Operator}

This is an issue that was originally raised by the NRC staff, and we defer to them regarding the applicant's response in Appendix G. We add a few comments here just to note our perspective on the role of the operator.

While it is desirable to design a reactor system to be resistant to operator errors, and perhaps even to provide safety that is independent of operator actions, this may not be possible. Because the PRISM heat removal systems perform so well under natural circulation conditions, the design does have a major advantage in decreasing the role of the operator. Similarly, the passive ("inherent") reactor shutdown mechanisms reduces the need for an operator assisted scram. However, there are some limitations in the passive shutdown mechanism, and the positive reactivity worth from sodium voiding is a major concern. Therefore, we believe that as long aS PRISM has a large positive sodium void reactivity worth, that GE will have to protect the operator and assure that the reactor can be shut down quiskly, if necessary.

Key Issues/Concerns in Section 4.12

- The operator's safety role in PRISM should be much reduced compared to LWRs, but it would be a mistake to assume the operator is not important to safety. In particular, we believe the operator must have the capacity to scram the reactor, to protect against the extremely improbable reactivity events.

\subsection{Multi-Module Control}

This is an issue that was originally raised by the NRC staff, and we defer to them regarding the applicant's response in Appendix G. We add a few comments here just to note our perspective on multi-module control.

Multi-module control is a very interesting technical challenge, especially with the increasing interest in automated control systems and so-called "expert systems". We would envision a fairly large automated control system being designed to manage a 9-module PRISM plant. The problem will be the size and complexity of this system, which would make an independent verification a formidable undertaking. The preferred solution would be to assure that the safety function of the plant is independent of the control system, and that the safety features/systems can protect the plant regardless of what the control system might do to cause problems. While this may also seem a formidable task, there are several features of the PRISM design that will go a long way toward assuring that this protection function is an inherent feature of the design.

Key Issues/Concerns in Section 4.13

- The vendors choice of a large digital control system for a multi-module PRISM plant seems entirely reasonable, i.e., it is a logical extension of current technology. It will be a formidable undertaking for anyone to independently assess such a massive and complex piece of software. For the moment we are recommending that the preferred course is to show the PRISM Reactor Module can withstand any challenges resulting from control system malfunctions. 


\subsection{Security}

This is an issue that was originally raised by the NRC staff, and we defer to them regarding the applicant's response in Appendix G. We add a few comments here just to note our perspective on security.

Of the items originally listed by the staff as priority items for improvement, the one we would most like to see implemented is a passive scram system, such as a suitable Curie-point magnet, or a thermal expansion based scram system. We believe that there may be some limitations associated with relying upon the passive reactor shutdown, based on reactivity feedbacks, in resisting an attempt to sabotage the plant.

Key Issues/Concerns in Section 4.14

- Our concerns have been registered through the security specialists.

\subsection{Prototype Tests}

While there is almost universal agreement that prototype testing is very valuable, and a major step toward design certification, there remains disagreement about how prototypic the tests must be and which types to tests can be omitted, e.g., a core meltdown. These issues could apply to several advanced reactor concepts.

In the original PSID [Ref. 1], the applicant indicated that the prototype might utilize an air-dump heat exchanger rather than a much more costly steam generator system (and sodium-water reaction pressure relief system). The applicant's argument was, and remains, that it is the passive reactor shutdown and heat removal that requires demonstration, and that the steam generator is simply a heat removal system that has little bearing on the safety of PRISM. These arguments seem quite reasonable, although the behavior of the air-dump system will be somewhat different than that of the steam generator system. Certainly it is the prototypic testing of the passive reactor shutdown mechanism that seems most crucial, and any problems created by substituting an air dump heat exchanger do not seem insurmountable at this time.

In response to the comments in the SER [Ref. 2], the applicant provided a preliminary list of prototype safety tests in Table G.4.15-1 and a list of events to be evaluated by analysis and laboratory testing in Table G.4.15-2. The list of prototype tests includes several conventional startup tests, several key tests of the passive reactor shutdown and passive decay heat removal systems, some seismic testing, and some surveillance activities. The list of passive system tests appears to be fairly complete, although we might recommend one or two variant cases, perhaps focusing on the pump coastdown devices. Some of the events listed in Table G.4.15-2 are related to steam generator failure and the more demanding seismic testing, although there is a lengthy station blackout event listed in the table. Certainly if there is a separate steam generator test facility, one could perform far more challenging tests, as one would be willing to risk damage to the IHX, for example. In addition, steam generator testing could be performed in parallel with the prototype reactor testing. The plan to perform more challenging seismic testing separate from the prototype may be based on practicality. A seismic test facility large enough to run tests on a full-scale PRISM module would be very large and probably impractical. With respect to the "station blackout" event without scram, there is probably somelogistical constraint involved here, as we don't know of any reason why this event could not be run on the prototype. 
We believe that the proposed prototype tests are valuable and that it may be a mistake to insist on large expensive systems that play only a minor role in the safety of PRISM. However, it is our opinion that the NRC staff should plan on full participation in the proposed tests, and should make every effort to stay involved in developing the test program.

Koy Issues/Concerns in Section 4.15

- The prototypicality of the test facility and the completeness of the test series will be the major issues, regardless of the design details or the testing procedures. Rather, the objectives will be to make the facility as prototypical as possible and to perform a comprehensive series of acceptable tests. With respect to PRISM, it is very improbable that large seismic events, core melt events, or over-power excursions, would be simulated. However, there are many important tests that should be performed, so the current concern is that the test facility and series are as complete and comprehensive as is practical.

\subsection{Safety Analyses}

This section focuses mostly on revisions to the Bounding Events analyses, particularly those events pertaining directly to the reactor. However, the last two subsections in Section G.4.16 [Ref. 1] discuss the newly added GEMs and Control Rod Stops, and how these may impact on the safety of the PRISM system.

\subsubsection{SER Positions and Pertinence of Design Changes}

In the draft SER [Ref. 2], some concerns were expressed regarding the expected PRISM system response to some of the postulated "Bounding Events", particularly in light of PRISM's containment design and the stated objective of avoiding the need for pre-planned off-site emergency evacuation procedures. Because our previous evaluations of the Chapter 15 events and the postulated BDBEs ("Beyond the Design Basis Events") [Ref. 3], did not indicate major problems, it was the Bounding Events that stood out as potential problems. Thus, GE chose in Section G.4.16 to address only the Bounding Events, and to focus on how changes in the PRISM design and newer information on the metal fuel performance impacts on the outcome of the postulated Bounding Events. It is noted that these changes would also change the analyses of events analyzed in Chapter 15 and Appendix $\mathbb{E}$ (the BDBEs). However, the safety margins for the Chapter 15 events were quite large previously and it is unlikely that these margins would be reduced significantly by any of the recent changes. In the case of the BDBEs, there is considerable overlap between these events and Bounding Events $1 \mathrm{~A}, 1 \mathrm{~B}$, and 2 (as interpreted by $G E$ as an unscrammed event), and GE's decision to focus on the Bounding Events rather than the BDBEs covered in Appendix $\mathrm{E}$ is acceptable.

GE correctly acknowledges that there were four Bounding Events of Concern, namely the UTOP with RVACS providing the only cooling (1b), the long adiabatic heatup event (3), the ULOF missing one pump coastdown (4), and the fuel assembly blockage (7). The first three cases are discussed in the sections to follow. The last item, involving the flow blockage, is discussed in Section 4.6.

\subsubsection{Summary of GE's Revised Results and BNL Interpretation}

GE summarizes their analyses of the bounding events in Section G.4.16.2, particularly in Table G.4.16-1. For Bounding Events 5 through 7, GE refers the reader to Section G.4.8.3 for the Steam Generator Tube Rupture (Event 5), Amendment 11 [also Ref. 1] for Large Sodium Leaks (Event 6), and Section G.4.6 for the postulated Assembly Flow Blockage (Event 7). GE defers their response for 
"External Events" (Event 8) "until receiving further input from the NRC staff" (i.e., a list of postulated external events).

Contained in Table G.4.16-1 are peak cladding and coolant temperatures, the peak mixed-mean core outlet sodium temperature, the estimated cladding loss (mils), and the minimum margin to sodium boiling for each of the first few bounding events, i.e, Bounding Events $1 \mathrm{a}, 1 \mathrm{~b}, 2,3 \mathrm{a}, 3 \mathrm{~b}$, and 4 . GE did not include the peak fuel temperatures in this table, which is consistent with ANL's stated position that it is the potential failure mode via cladding failure that is the greater concern, and the ANL's position that a little localized fuel melting during highly improbable events is acceptable. Regardless, it is noted that the peak fuel temperatures during Bounding Events $1 \mathrm{a}$ and $1 \mathrm{~b}$ (the unprotected transient-over-power cases) are indeed above the solidus temperature.

In order to better comprehend key portions of Table G.4.16-1 and other information contained within Section G.4.16, we constructed Figure 16. Included are the peak fuel, cladding, and coolant temperatures from GE's analyses for Bounding Events $1 \mathrm{~A}, 1 \mathrm{~B}$, and $2^{\prime}$ ( $\mathrm{GE}$ analyzed a more challenging variation on the bounding Event 2 recommended by the NRC staff), and 4 , along with some key information regarding failure limits (see also Section 4.16 .3 .2 regarding appropriate limits). In the interest of presenting a good overview on one figure, we have taken some liberties with the cladding damage range and the fuel solidus temperature range, and have packed more than the optimum amount of information into this single figure. However, the array of information provided by ANL regarding metal fuel performance and failure modes is a little overwhelming, and we feel that Figure 16 is not inconsistent with the information we have been provided (see also Section 4.16.3.2).

It is clear from Figure 16 that some fuel would be heated above the solidus temperature for Bounding Events $1 \mathrm{~A}$ and $1 \mathrm{~B}$. For the UTOP events, the power production in the pins increase by about $70 \%$, so the pin centerline temperatures can increase very substantially. As the peak fuel temperatures for $1 \mathrm{~A}$ and $1 \mathrm{~B}$ are above the solidus temperature for relatively high $\mathrm{Zr}$ fuel, there is little doubt that some localized melting would take place. Some policy decision will be required here, in terms of how much localized fuel melting will be acceptable.

If we focus on the peak cladding and sodium temperatures for events $1 \mathrm{~A}$ and $1 \mathrm{~B}$ we can see the system gets significantly hotter if only RVACS is available to remove the heat. These higher temperatures are needed to radiate the heat out though the vessels, and result in a lower power production in the core. As a result, the increase in fuel temperatures is significantly less than the increase in cladding and sodium temperatures. For Bounding Event $1 B, G E$ is estimating a cladding wastage of 0.22 mils (Table G.4.16-1 from Ref. 1), which is about 1\%. This amount of damage, if correct, would probably be acceptable for such an unlikely event, (further discussed in Section 4.16.3.3.1.2). The peak sodium temperature is well below boiling, and does not appear to be a problem for the UTOP events.

The ULOF-ULOHS (Event 2') and ULOF-3/4 Coastdown (Event 4) events on the right side of Figure 16 result in significantly lower fuel temperatures. There may be a very slight amount of cladding damage for these events, especially if conservative limits (Section 4.16.3.2) are applied. The sodium is well below boiling, with or without pumping. A major factor in limiting the peak temperatures is the use of the GEMs to insert a large amount of negative reactivity once the pumps trip off line.

Thus, the GE results presented in Section G.4.16.2, as summarized in Figure 16 point directly to the postulated UTOP events as the safety concerns within the category of "Bounding Events". This will be discussed in subsequent sections. However, our independent analyses tend to confirm this, although our peak fuel temperatures are somewhat higher, and the subsequent cladding damage greater. 


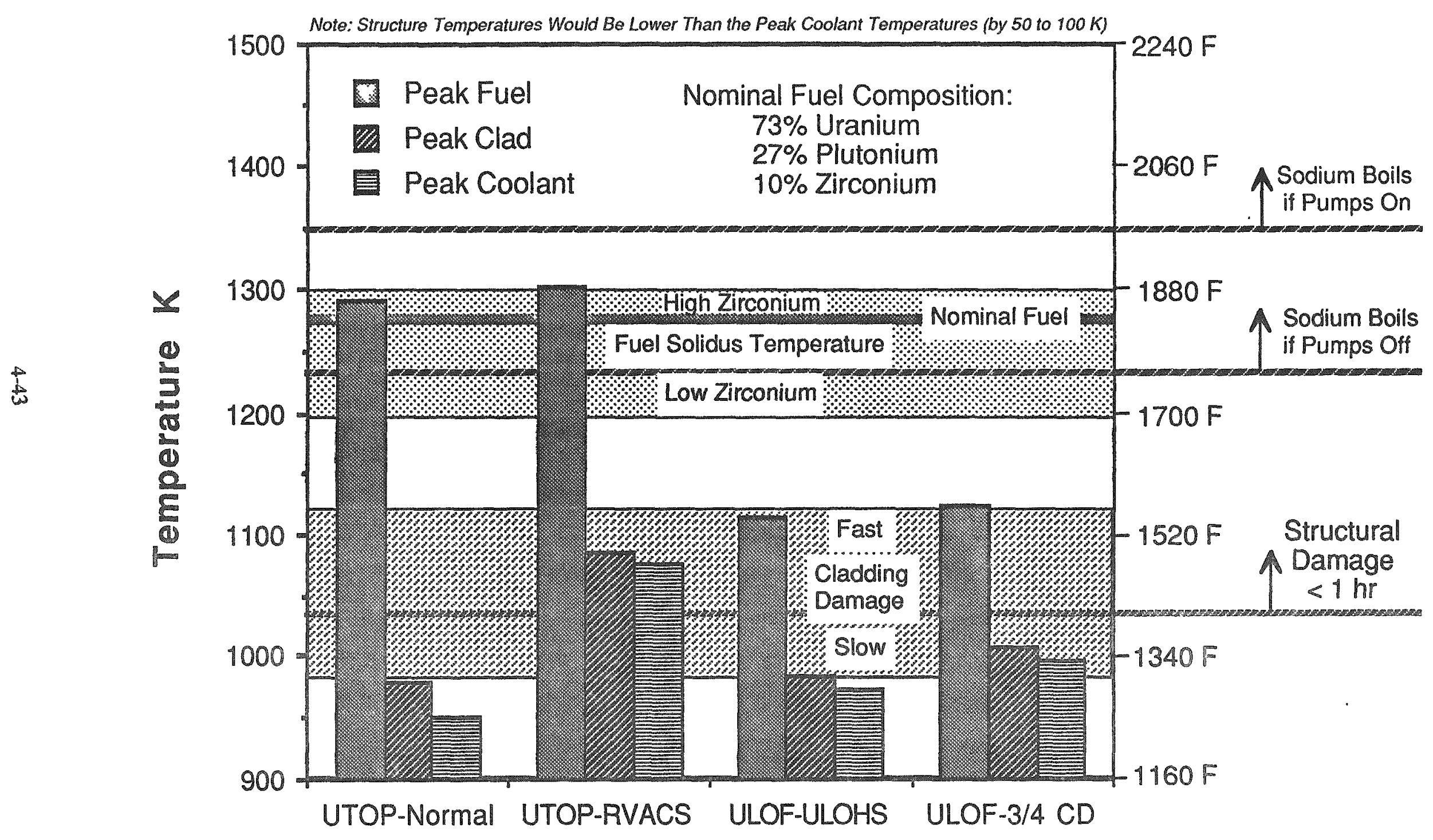

\section{Bounding Events 1A, 1B, 2', and 4}

Figure 16. Peak Fuel, Clad, and Sodium Temperatures for 4 Key Bounding Events and Pertinent Limits for Fuel, Cladding, Structures, and Sodium Boiling. 
Despite ANL's optimism that they can demonstrate the metal fuel can survive these events, GE may have to consider reducing the potential UTOP initiator below 40 cents (including uncertainties), perhaps by moving the control rod stops more frequently. Because the applicant seems to have a few options available to reduce the potential UTOP initiators, the results summarized in Figure 16 are not viewed as an insurmountable problem.

\subsubsection{Revised Analyses of Core-Related Bounding Events (1 through 4)}

We revised our independent analyses of several postulated events, including Bounding Events $1 \mathrm{~A}$, $2,3 \mathrm{~A}, 3 \mathrm{~B}$, and 4 . The analysis of Bounding Event $1 \mathrm{~B}$ will require some model modifications, which have not as yet been implemented. However, we have enough direct comparisons between our analyses and those provided by GE to project the likely results for $1 B$. Because GE had an error in representing the reactivity insertion due to control rod drive-line expansion (their rod worth was too high), their predictions for all the unscrammed events are slightly cooler ( $a$ few degrees $K$ ) than they should be. Thus, we expect GE's results for Bounding Event $1 \mathrm{~B}$ are fairly accurate, although the fuel should be slightly hotter and a little more cladding damage should be expected.

\subsubsection{Analytical Approach}

Most of the BNL independent analyses were performed using two well established codes, i.e., the SSC [Ref. 21] and MINET [Ref. 19 and 20] Codes. SSC was developed for analyzing various LMR systems under transient conditions. However, it was necessary to add some models to SSC for analyzing the PRISM system, as is discussed in the next section. MINET is a highly flexible systems code that could be utilized to analyze the postulated long-term heat-up events, as well as features of the pump coastdown events. Between SSC and MINET, most of the modeling requirements were fulfilled. Any gaps were covered using special purpose models. It is noted that work is in progress to reconfigure SSC, MINET, and other analytical tools to create a more complete integral representation of the PRISM system.

\subsection{SSC Modeling [Ref. 21]}

A full-plant SSC model was used to represent PRISM in our independent analyses, as illustrated in Figure 17. Several major components were represented, as shown. For the reactor, seven channels were used to represent the drivers, the internal blankets, the radial blankets, the control assemblies, the reflector region, the shield assemblies, and a hot driver assembly. The bypass flow was also modeled. Twelve axial nodes were used for each assembly, with two nodes utilized for the lower shield region, six nodes for the active core, and four nodes used for the gas plena. Each axial node includes four radial rings in the fuel region plus one for the cladding.

Data utilized for representing the IHX, the pumps, the steam generators, and other key coolant system components were taken from the PSID [Ref. 1] or were obtained directly from the applicant. The EM pump representation was simplified, as SSC has no explicit provision for representing the EM pump. (Such a model has been developed and tested in MENET, but has not yet been incorporated into SSC). Therefore, the primary system flow rate was imposed as a transient boundary condition, based on calculational results from both GE's analyses and results we generated using the MINET Code.

SSC was originally developed to analyze oxide fuel LMRs. To facilitate modeling of the metal fuel utilized in PRISM, several modifications were implemented, as documented in References 3 and 22. For the most part, these modifications could be utilized to analyze the revised PRISM core design. The principal change was the addition of a model for the Gas Expansion Modules (GEMs). Three of these 


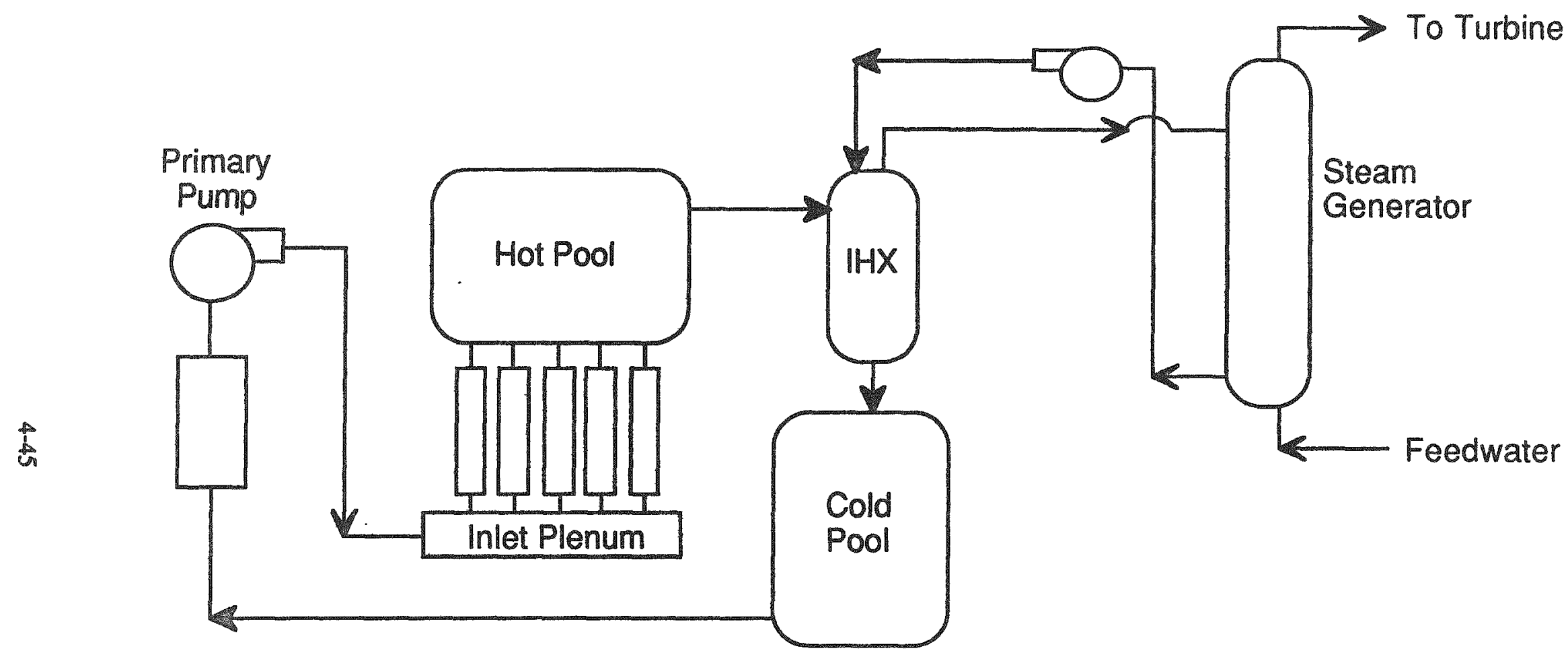

SSC Representation of PRISM Reactor System

Figure 17. SSC Representation of PRISM Reactor System. 
assemblies were added to PRISM in order to supplement the negative reactivity feedback that develops once the pumps have been tripped. When the pumps trip and the pressure drops, the sodium within the GEMs at the active core elevation is displaced by helium gas, thus increasing the leakage of neutrons from the core and subtracting about $69 \mathrm{c}$ of reactivity, assuming all three GEMs function properly. The operational mode of the GEMs is illustrated in Figure 18.

The GEM is essentially an empty assembly duct, sealed at the top, open at the bottom and connected to the high pressure in the inlet plenum of the core. A hexagonal cross section duct, with a wall thickness slightly greater than the standard fuel and blanket duct, forms the unit. When the pumps are at full flow, the plenum pressure (minus the static head to the GEM level) compresses the gas in the GEM cavity to be above the core. This causes more neutrons to be scattered and deflected back into the core, as compared to when the gas is adjacent to the core. When the flow decreases, the trapped helium expands and drops the sodium level into the core region. As a result, fewer neutrons are scattered back into the core region. The reactivity effect increases as the gas expands into the core and remains constant once the gas liquid interface drops below the core region. At this point the maximum negativity reactivity. of 69 (i.e. 23 c each) is imposed.

In SSC, three equations are solved iteratively until they converge to give the correct sodium level in the GEMs. They are:

$$
\begin{gathered}
v t=v_{\ell}+v g \\
P g-\rho * g * h_{\ell}=\text { PCi } \\
P g * V g=M g * R * T
\end{gathered}
$$

where

$\begin{array}{llll}\mathrm{Vt} & = & \text { total GEM volume } & (\mathrm{m} * * 3) \\ \mathrm{V}_{\ell} & = & \text { GEM sodium volume } & (\mathrm{m} * * 3) \\ \mathrm{Vg} & = & \text { GEM gas volume } & (\mathrm{m} * * 3) \\ \mathrm{Pg} & = & \text { GEM gas pressure } & (\mathrm{Pa}) \\ \mathrm{Pci} & = & \text { Core Inlet Plenum pressure } & (\mathrm{Pa}) \\ \rho & = & \text { sodium density } & \left(\mathrm{kg} / \mathrm{m}^{* * 3}\right) \\ \mathrm{g} & = & \text { gravity } & \left(\mathrm{m} / \mathrm{s}^{* * 2}\right) \\ \mathrm{A} & = & \text { GEM area } & (\mathrm{m} * * 2) \\ \mathrm{h}_{\ell} & = & \text { sodium level in GEM } & (\mathrm{m}) \\ \mathrm{Mg} & = & \text { Mass of Helium in } \mathrm{GEM} & (\mathrm{kg}) \\ \mathrm{R} & = & \text { helium gas constant } & (-) \\ \mathrm{T} & = & \text { GEM gas temperature }(\mathrm{K}) & \end{array}$

The gas temperature closely follows the GEM shell temperature which is determined by tracking the heat transfer between the neighboring assemblies and the GEM 


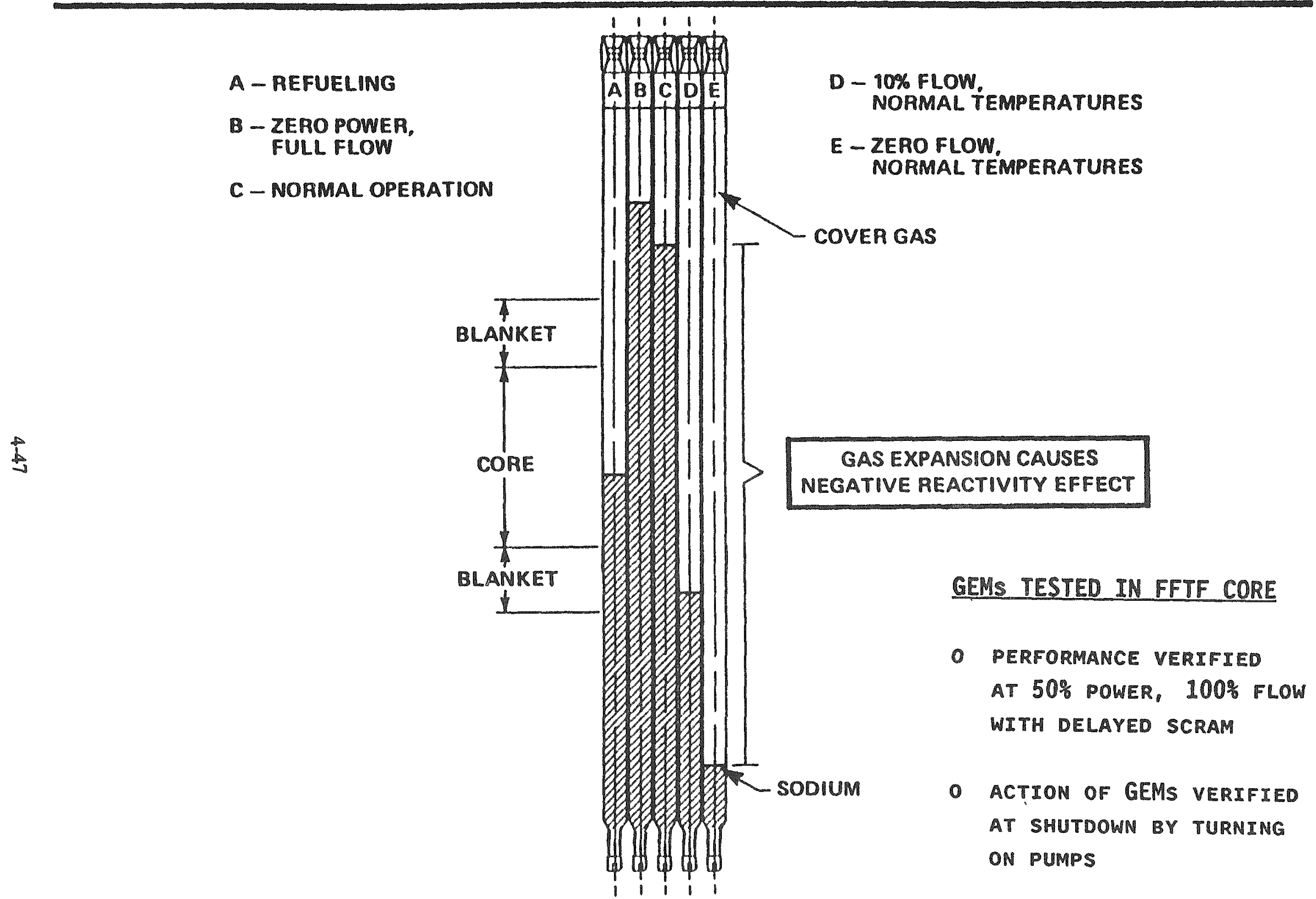

Figure 18. Operation of the Gas Expansion Module (GEM) Tested in FFTF (Which has a Similar Behavior in PRISM). 


$$
\mathrm{Cp} * \mathrm{Mg} * \mathrm{dT} / \mathrm{dt}=\mathrm{Q}
$$

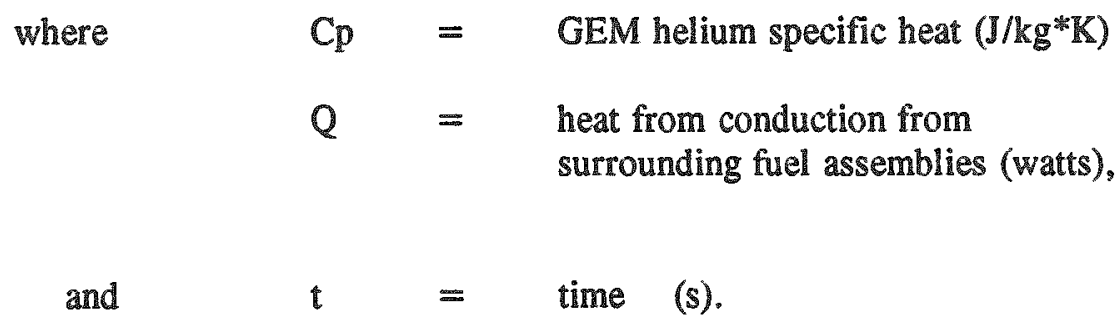

These equations are solved at each time step to determine the sodium level in the core. The worth of the GEMs when the level is equal to, or greater than, the top of the core is zero. When the level reaches the bottom of the core, the GEMs are worth -69 cents. Intermediate values of reactivity are interpolated linearly from the liquid level in the GEMs.

\subsection{MINET Representation [Ref's. 19 and 20]}

The MINET Code is a highly flexible and modular systems code which is based on a momentum integral network formulation [Ref. 20]. While several different system representations were utilized in analyzing PRISM, there were all variations on the layout shown in Figure 20. The core representation includes user-specification of the heating term (decay heat once scram occurs), as the reactivity feedback modeling currently available in MINET is not sufficiently detailed for analyzing the passive shutdown mechanism. Models for the pipes and pools conserve mass, energy, and momentum alorig a length of piping (called segments). The pump representation was extended to model the PRISM EM pumps, including the coastdown response, in detail. The IHX was simulated as a full heat exchanger in some cases, and as a user-input "heated pipe" in other cases. The RVACS heat loss was specified as a time/temperature dependent heat loss in volume 108. Some auxiliary piping and valve modules were utilized to facilitate simulation of postulated pipe rupture events. As a result, the valve modules identified as 501 and 502 on Figure 19 have no physical equivalent in PRISM, and they are present only so the user can allow the sodium flow to leak or close off.

While the MINET models could be used to simulate several types of transient events, the applications thus far have focused in two areas. First, and most extensively, MINET has been used to simulate long term heat-up events. During these events a scram occurs, and normal and ACS cooling are lost, leaving only the RVACS to remove the afterheat. After a few hours, the sodium becomes hot enough for the RVACS spill-over to occur, which means transferring sodium from volumes 101 to 108 in the MINET representation illustrated in Figure 19. Over the long term, the system gradually heats up to a degree where the heat loss to the up-flowing RVACS air equals (and then exceeds) the decay heat production, which may be a day or so after the event begins. The second MINET application has been for postulated pipe rupture and pump seizure (coastdown failure) events. A complex model of the EM pumps and the synchronous coastdown machines was implemented in MINET, and the results from these calculations are factored into Section 4.16.3.3.4.

\section{16.3.2 Damage and Failure Limits}

In section G.4.16.3.2 [Ref. 1], the applicant cites the damage and failure limits for the cladding, sodium, structure, and, to some degree, the fuel. For the sodium (boiling) and structure (ASME Codes) these limits are easily quantified. The situation for the HT9 cladding is not as clear, and involves both creep rupture and eutectic formation failure modes. For the ternary fuel, the limits are very hard to 


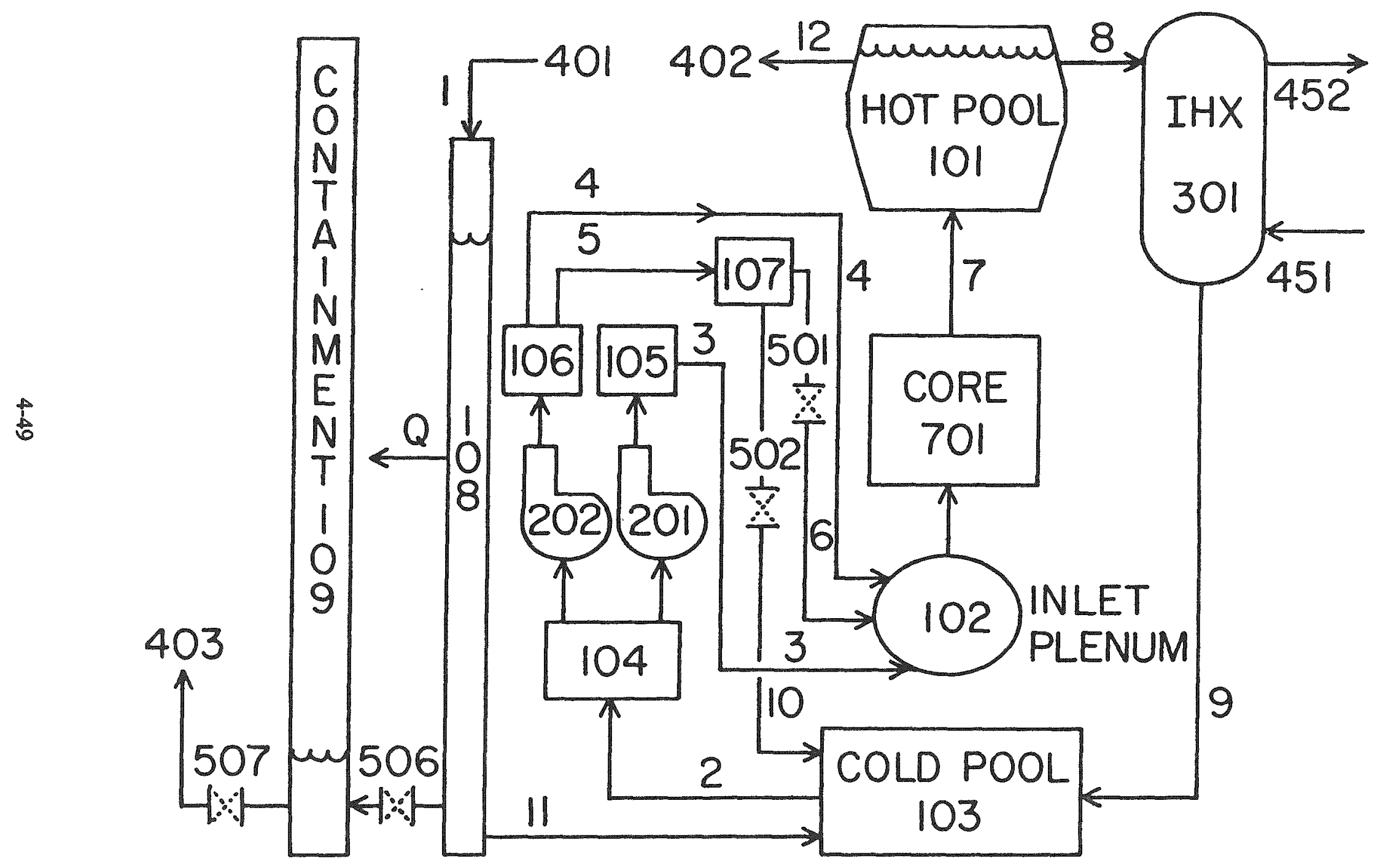

Figure 19. MINET Representation of PRISM System. 
qualify, and vary throughout the fuel lifetime and across each fuel pin. There is a time factor involved in some of the failure limits, especially the cladding and structural temperature limits.

\section{Sodium Boiling:}

The saturation temperature for the sodium depends on the pressure, which varies with sodium depth and pumping (increases system pressure and pressure drops). In PRISM, the in-core sodium boiling temperature is about $1344 \mathrm{~K}(1960 \mathrm{~F})$ if the pumps are operating and $1233 \mathrm{~K}$ $(1760 \mathrm{~F})$ if the pumps are off.

\section{Structurail Integrity:}

The ASME Code Level D limits are $1033 \mathrm{~K}$ (1400 F) over the short term (less than an hour) or $977 \mathrm{~K}(1300 \mathrm{~F})$ over the longer term, i.e., more than an hour. In general, the structural temperatures will be similar to the reactor outlet sodium temperature, although they will lag the sodium temperature significantly during the early portion of a transient.

\section{Cladding Failure:}

HT9 has some excellent properties, especially with respect to surviving in a high neutron flux and energy environment. However, at elevated temperatures, HT9 loses some of its creep strength and also begins to interact with the fuel to form a low-melt temperature eutectic. The applicant has explicitly factored these failure modes into their analytical tools, and compares the cladding damage in mils against the nominal cladding thickness of 20 mils. As a preliminary design limit, the cladding attack has been limited (by GE) to less than $10 \%$ of the wall thickness, i.e., to 2 mils. The applicant's analyses of the bounding events show a maximum cladding attack of 0.22 mils for Bounding Event $1 \mathrm{~B}$ and a trivial amount for the other bounding events. (Figure 16 only shows the peak temperature and not the duration at the indicated values. Most of the transients are fast, so the integral damage is correspondingly small).

\section{Fuel Melting:}

GE's and ANL's position is the following:

"Fuel melting, per se, is not a cause of pin failure. TREAT tests have demonstrated that extensive fuel melting does not affect the basic pin failure mechanism. Failure by cladding creep rupture, with clad thinning by fuel-clad liquid phase formation, is the appropriate mechanistic cladding breach criterion..."

If the relocation of fuel into the coolant channel and beyond were the sole concern, we would tend to agree with this position. However, the PRISM reactor is not configured to give the highest possible reactivity. Movement of a significant amount of fuel towards the center of the core would increase reactivity and could lead to severe damage. The reference metal fuel has only $75 \%$ smear density as well as a gap between the fuel and cladding at low burnups. Molten fuel can relocate within the clad which could cause an increase in reactivity for the core.

\section{Fuel and Cladding Behavior and Uncertainties:}

Both the ternary metal fuel and the HT9 cladding are in a development stage, and there is little data available at significant burnup levels. Therefore, the temperature limits (eutectic tormation and perhaps others) are not really well known. Since ANL has been working with metal fuels 
for over three decades, their estimates regarding fuel performance must be considered expert opinion at this time. There are several areas where more data is required and they are:

1) Fuel-cladding chemical interaction forms a eutectic with a minimum melting temperature at the fuel-clad interface, caused mainly by iron (Fe) diffusion into the fuel. This effect is compounded by lanthanide migration, plutonium migration, zirconium migration and the kinetics at the fuel/clad interface. This minimum eutectic temperature must be determined for the prototypical fuel.

2) The maximum fuel-clad liquid penetration rate from the eutectic formation must be determined from irradiated fuel, where the lanthanide, zirconium, plutonium, and iron diffusion is accounted for.

3) The impact of lanthanide penetration into the cladding.

4) The migration of the fuel components to form multiple annular zones having too much or too little uranium, zirconium, or perhaps plutonium (the plutonium migration appears to be modest).

5) Estimation of fuel solidus and liquidus temperatures depends on component migration with burnup, and this has not been fully characterized. Similarly local thermal conductivity and expansion will depend on component migration.

6) The impact of fuel reprocessing contamination on fuel composition and performance needs better resolution, especially regarding its impact on thermal conductivity, isotope migration, fuel-clad interaction, and zone formation.

7) The EBR-II metal fuel data was collected on samples with a high volumetric heating rate. Data at different heating rates are needed to determine if this gives a conservative estimation of the fuel's failure mechanisms.

We believe that a conservative assessment of literature available on the eutectic data and physics suggests a cladding temperature limit of about $900 \mathrm{~K}$, about $75 \mathrm{~K}$ lower than G.E. assumes. However, throughout much of this report we have compared against the applicants limits of $980 \mathrm{~K}$, on the assumption the next batch of data will support ANL's current best estimate. It must be recognized that this fuel is still under development and evaluations are based on currently available data.

\section{BNL Estimation of Eutectic Formulation:}

The data collection for the eutectic temperature associated with reactions of $\mathrm{U}-26 \mathrm{Pu}-10 \mathrm{Zr}$ with HT9 clad has only started since PRISM's fuel was changed from U-19 Pu-10Zr recently. As discussed below, the data can only substantiate the limit at $903 \mathrm{~K}$. It is possible that future EBR-II irradiated specimens may demonstrate an effectively higher eutectic threshold due to fuel surface effects which reduce $\mathrm{Fe}$ diffusion.

The only data currently available to address this effect are the Differential Thermal Analysis (DTA) and the Diffusion-Couple (DC) tests. Both tests use only un-irradiated fresh fuel. In DTA tests, a section of clad topped by a piece of fuel was placed in an yttrium crucible and heated to $1823 \mathrm{~K}$. The material was fully molten at $1573 \mathrm{~K}$. The specimens were slowly cooled, and exothermic reactions identified precipitation of $\mathrm{ZrFe}_{2}$ between $1323 \mathrm{~K}-1373 \mathrm{~K}$ and a final solidification between $883-923 \mathrm{~K}$. 
In the DC tests, alternating sections of fuel and clad were sandwiched into a molybdenum holder and heated at constant temperature and examined for melting. These tests showed melting for the PRISM type fuel at approximately $903 \mathrm{~K}$.

Based on this information (which doesn't include surface effects, irradiation effects or kinetic factors) the eutectic temperature for U-26 Pu-10Zr fuel with HT9 clad must be assumed to be $903 \mathrm{~K}$ until more data are collected.

The DTA data probably represent equilibrium behavior for the system $\mathrm{Fe}, \mathrm{U}$, and $\mathrm{Pu}$ at a series of U/Pu ratios. The high temperature reaction of $\mathrm{Zr}$ with $\mathrm{Fe}$ and/or $\mathrm{Cr}$ to form the stable intermetallic compound $\mathrm{Fe}_{2} \mathrm{Zr}$, effectively removed the $\mathrm{Zr}$ from the system. The subsequent cooling curves were for a mixture of $\overline{\mathrm{Fe}}, \mathrm{Pu}$, and $\mathrm{U}$. Fe forms low-melting eutectics with $\mathrm{U}(\mathrm{m} . \mathrm{p} .=998 \mathrm{~K}, 66 \% \mathrm{U})$ and $\mathrm{Pu}$ (m.p. $=683 \mathrm{~K}, 90 \% \mathrm{Pu}$ ). For a first approximation, we can assume the minimum melting point of ternary Fe-U-Pu alloys lies along a line connecting these two points. ANL DTA data show the eutectic temperature decreases with increasing Pu, consistent with this model. For the reference alloy 64U-26Pu-

$10 \mathrm{Zr}$, an estimated melting point would then be $\left[\frac{.64 \times 998+.26 \times 683}{0.9}\right]$, or $907 \mathrm{~K}$, in good agreement with the experiment.

However, for melting to occur at this eutectic temperature the $\mathrm{U}, \mathrm{Pu}$, and $\mathrm{Fe}$ must come into intimate contact in this ratio. When heating a fuel/clad mixture, $\mathrm{Zr}$ does not get removed from the reaction by precipitation with $\mathrm{Fe}$ or $\mathrm{Cr}$ until much higher temperatures are reached. $\mathrm{Zr}$ may also concentrate at the fuel/clad interface by reacting with the $\mathrm{Fe}$ or $\mathrm{Cr}$ to form $\left(\mathrm{Fe}, \mathrm{Cr}{ }_{2} \mathrm{Zr}\right.$ and/or $\mathrm{Zr}(\mathrm{C}, \mathrm{N})$ at the interface. These can act as diffusion barriers to eutectic melting reactions, and delay their onset as the temperature is raised. Fission products in irradiated fuels, especially lanthanides, may also affect both the thermodynamics and kinetics of the melting process. The system is highly complex and not readily explained in detail.

A collection of measured Fuel Behavior Test Apparatus (FBTA) test data was used to develop the ANL correlation for cladding penetration. However, the data are (mostly) based on binary fuel or ternary fuel with Type 316SS cladding. The component makeup of HT9 indicates that the penetration rate might not be similar to the previous data with D9 or 316SS in binary, or ternary fuel. Only three data points from U-19Pu-10Zr with HT9 at $3 \%$ burnup were reported to date:

$\begin{array}{lc}\text { Rate }(\mu \mathrm{m} / \mathrm{s}) & \text { Temperature }(\mathrm{K}) \\ 0.018 & 1053 \\ 0.0146 & 1023 \\ 0.009 & 973\end{array}$

They fall on a straight line. This correlates to $R(\mu \mathrm{m} / \mathrm{s})=\exp [2.05 \mathrm{~T}(\mathrm{~K})-5289.6 /(\mathrm{T}(\mathrm{K})-273)]$ which is lower at higher temperature, but higher at lower temperatures than the ANL correlation. At $903 \mathrm{~K}$ the ANL correlation gives $4.3318 \mathrm{E}-4 \mu \mathrm{m} / \mathrm{s}$ (or 32.6 hours to penetrate $50.8 \mu \mathrm{m}$ of clad) and the above correlation gives $4.247 \mathrm{E}-3 \mu \mathrm{m} / \mathrm{s}$ (or 3.32 hours to penetrate $50.8 \mu \mathrm{m}$, which is G.E's definition of cladding failure). More data are needed with prototypical fuel and clad before final conclusions can be made.

It must be noted that the evaluation of the eutectic data base for U-26 Pu-10Zr with HT9 has just started. Due the different chemical make up of Type 316SS and HT9, data collected with Type 316 SS 
cannot be assumed to apply to HT9 without more analysis. A pin with $2.3 \%$ burnup from EBR-II has recently been evaluated in the FBTA. It was found to have an onset of clad/fuel interactions at a temperature between 1023 and $1073 \mathrm{~K}$. However, this effort contributed only a few data points and was at too low a burnup to typify the effects of Fe interdiffusion, fission product migration, and effects from prolonged contact between the fuel and clad. The data of interest will be between 10 and $15 \%$ burnup, which will not be available for a few years.

\subsubsection{Analyses of Individual Bounding Events}

As mentioned previously, GE analyzed postulated events that were largely consistent with those listed by the NRC staff as Bounding Events 1A, 1B, $23 \mathrm{~A}, 3 \mathrm{~B}$, and 4. The BNL calculations utilized entirely different analytical tools, as well as a few variant sets of assumptions. In most cases, we have results that should and do closely correspond to the results provided by GE. In some of the variant cases, we have used more conservative assumptions or slightly altered assumptions regarding system performance. As these variant case often involved more conservative assumptions, the safety margins are generally smaller than for the reference bounding events.

\subsection{Unprotected Transient-Over-Power Events (UTOPs)}

An unprotected transient over power (UTOP) accident results when positive reactivity is inadvertently inserted into the core and there is a failure to scram. The limiting case assumption is that all the control rods are accidently removed. This event is bounded by the amount of reactivity available in the control rods. In an oxide fuel core (i.e., U02), the temperature and power defect and built in excess reactivity for the burnup swing (excluding axial expansion) is generally several dollars worth, making the event very severe, should it occur. The metal fuel core in PRISM has, on the other hand, a small temperature and power defect $(\sim 1.2)$, negligible burnup swing (excluding axial expansion) ( $\sim \$ 0.04)$, and excess reactivity to account for the fuel axial expansion ( $\sim \$ 1.1)$. The amount of reactivity available for a UTOP is reduced by adding control rod stops in PRISM, such that only a limited amount of excess reactivity ( $\sim \$ 0.40$, including $\$ .10$ to cover uncertainties) can be added.

Because of the small Doppler feedback, the UTOP scenario can be very challenging for a metal fuel core. The hard neutron spectrum of a metal core has relatively few neutrons in the prominent U-238 resonances; thus, giving the PRISM reactor a small Doppler feedback. This is the usual mechanism to limit an over power event of the axial core. Instead, the PRISM design must rely on neutronic feedbacks from radial expansion, control rod expansion, and fuel axial expansion to limit the peak power. As the temperature increases, their impact on the neutronic reactivity feedbacks are relied upon to limit the energy production to an acceptable level.

\subsection{B.E. 1A: UTOP with Normal Cooling}

The event is initiated from full power. The control rods are assumed to begin withdrawing with a speed of $\$ 0.02$ per second. The control rod stops are supposed to limit the withdrawal worth to 30 cents. However, GE has adapted $40 \mathrm{c}$ as the TOP initiator to account for uncertainties and to be conservative. Thus, the PRISM TOP is assumed to insert 2 cents/s for 20 seconds, for a total of 40 cents, representing the withdrawal of all the control rods.

The initial conditions used and important system parameters are listed in Table 1. A majority of the initial conditions were taken from the PSID amendment for the 1989 version of PRISM, with the remainder from direct communications with the vendor. 
Table 1

Table of Initial and Key Operating Parameters

\begin{tabular}{llc} 
Description & $\underline{\text { PRISM }}$ & $\underline{\text { SSC }}$ \\
Power (MW) & 471 & 471 \\
Cover Gas (kPa) & 99.3 & 99.3 \\
Primary Flow (kg/s) & 2513 & 2507 \\
Primary Sodium Inlet (K) & 610.9 & 610.9 \\
Primary Sodium Outlet (K) & 758.1 & 758.0 \\
Inlet Plenum (kPa) & 744.6 & 744.6 \\
Pump Rise (kPa) & 614.3 & 614.3 \\
Assembly Length (m) & 4.978 & 4.978 \\
Core Height (m) & 1.3462 & 1.3462 \\
Peak Fuel Pin/Average Fuel Pin & 1.31 & 1.31 \\
Fuel Pin OD (m) & .00668 & .00668 \\
Driver Fuel Pins/Assembly & 5293 & 2275 \\
Intermediate Sodium Flow (kg/s) & 331 & 557.0 \\
Intermediate Sodium IHX Inlet (K) & 720.0 \\
Intermediate Sodium IHX Outlet (K) & 331 \\
\hline
\end{tabular}


Two UTOP cases were analyzed. The first calculation represented a case where SSC is set up to replicate the ARIES calculations discussed in the PSID. This was done to verify that the models give comparable results for similar assumptions. The second calculation represents the PRISM system if more conservative assumptions are made about the fuel behavior.

\section{Nominal Case: TOP Calculations with Nominal Fuel Properties}

The withdrawal of all of the control rods is represented by an insertion of 2 cents $/ \mathrm{s}$ for 20 seconds. The EM pumps are assumed to continue to operate at nominal conditions. The control rods are thus removed to the rod stop position without causing activation of the reactor protection system.

The plots from the analysis are shown in Figures 20 through 28. The power reaches a peak of 1.7 times rated by $23 \mathrm{~s}$, and begins to level off at 1.22 times rated by 7 minutes, as shown in Figure 20 . The increase in power increases the average core outlet sodium temperature (Figure 21) to a maximum of $870 \mathrm{~K}$. This increase in the core outlet sodium temperature also improves the performance of the IHX as shown in Figure 22. The total rise in core outlet temperature is $112 \mathrm{~K}$. Eventually the core outlet sodium temperature is re-established around $833 \mathrm{~K}$, which is $75 \mathrm{~K}$ above the initial temperature.

The changes in the reactivity feedbacks can be seen in Figures 23 through 25 . The total (net) feedback, shown in Figure 23, starts out positive because of the reactivity from the control rods being removed, but turns downward once the negative feedbacks increase enough to counter the positive insertion. The rise in fuel temperatures first increases the Doppler absorption of the neutrons, and then triggers the fuel's elongation. These two feedbacks add about $-23 \mathrm{c}$ at the time of peak power. Higher sodium temperatures create a harder neutronic spectrum, which generates a positive feedback of about +66 at the time of peak power. The higher sodium temperatures cause the thermal expansion of the control rod drive line and the radial expansion, as indicated in Figures 24 and 25 . The control rod drive lines have time constants of around $30 \mathrm{~s}$, and are slow to act as compared to the radial expansion. Radial expansion, shown in Figure 25, is composed of the grid plate and the above core load pad (ACLP) expansion, which are as shown in Figure 24. The radial expansion adds the crucial amount of reactivity that eventually limits the power increase to 1.7 times rated power, and then contributes to the power reduction that follows. The control rod driveline expansion reactivity continues to increase in worth, and drives the total reactivity to zero after around 80 seconds. Although the reduced power decreases the worth of several of the reactivities, the control drive line continues to expand, causing the total reactivity to become slightly negative and re-stabilizing the power near 1.22 times the rated level.

The predicted behavior of the component reactivities is very similar between GE's ARIES calculations and the SSC calculations. A comparison between the two predictions is shown in Table 2. One point of contention was the worth of the control rod driveline, which ARIES was predicting to reach a worth of about 30 cents, while SSC predicted about 20 cents. Upon investigation, the ARIES worth curve was found to have been set erroneously for the smaller (old) height core, and the BNL prediction was correct. The plots in the PSID will have to be modified by GE to correct this problem, but have not yet been replaced. Also, the ARIES modeling used the single assembly bowing model, which gives about 5 to 84 of negative reactivity that is not factored into the SSC calculation. This effect was intentionally left out of the SSC calculation, as the quantification of the radial bowing is very difficult and the feedback is always negative when the temperatures are rising, so omitting it is believed to be both conservative and prudent. (However, the bowing feedback will not be a significant issue until there is a case identified where one needs the bowing contribution to predict a safe response to a postulated event).

The results from the UTOP power spike and increase in power level on the fuel can be seen in Figures 26 through 28 , where the peak temperatures are shown. It must be noted that the thermal 


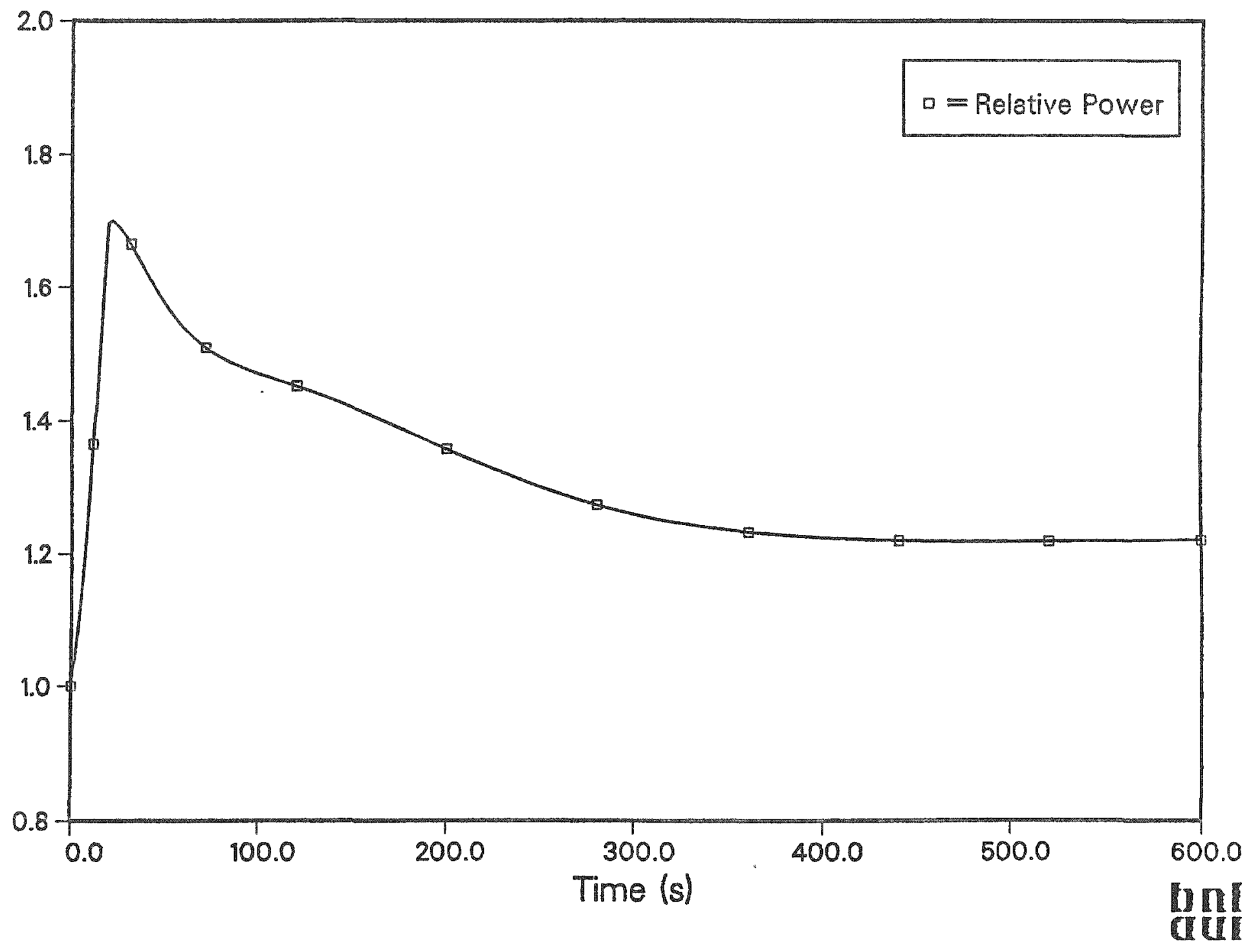

Figure 20. Relative Power Prediction from SSC for PRISM During a 40 Cent UTOP for Assumptions Similar to ARIES (i.e., GE Code). 


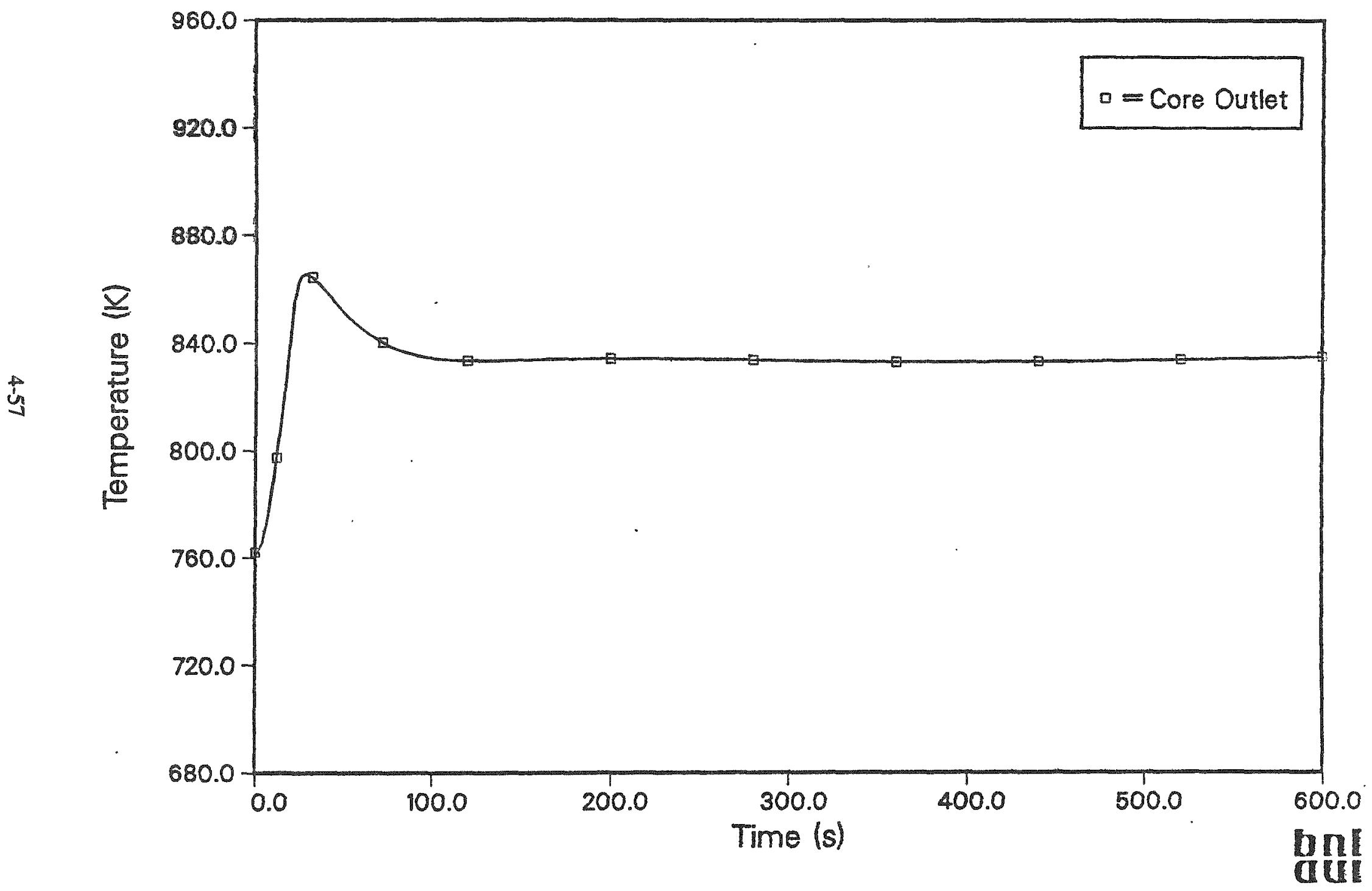

Figure 21. Average Core Outlet Temperature Prediction from SSC for PRISM During a 40 Cent UTOP for Assumptions Similar to ARIES. 


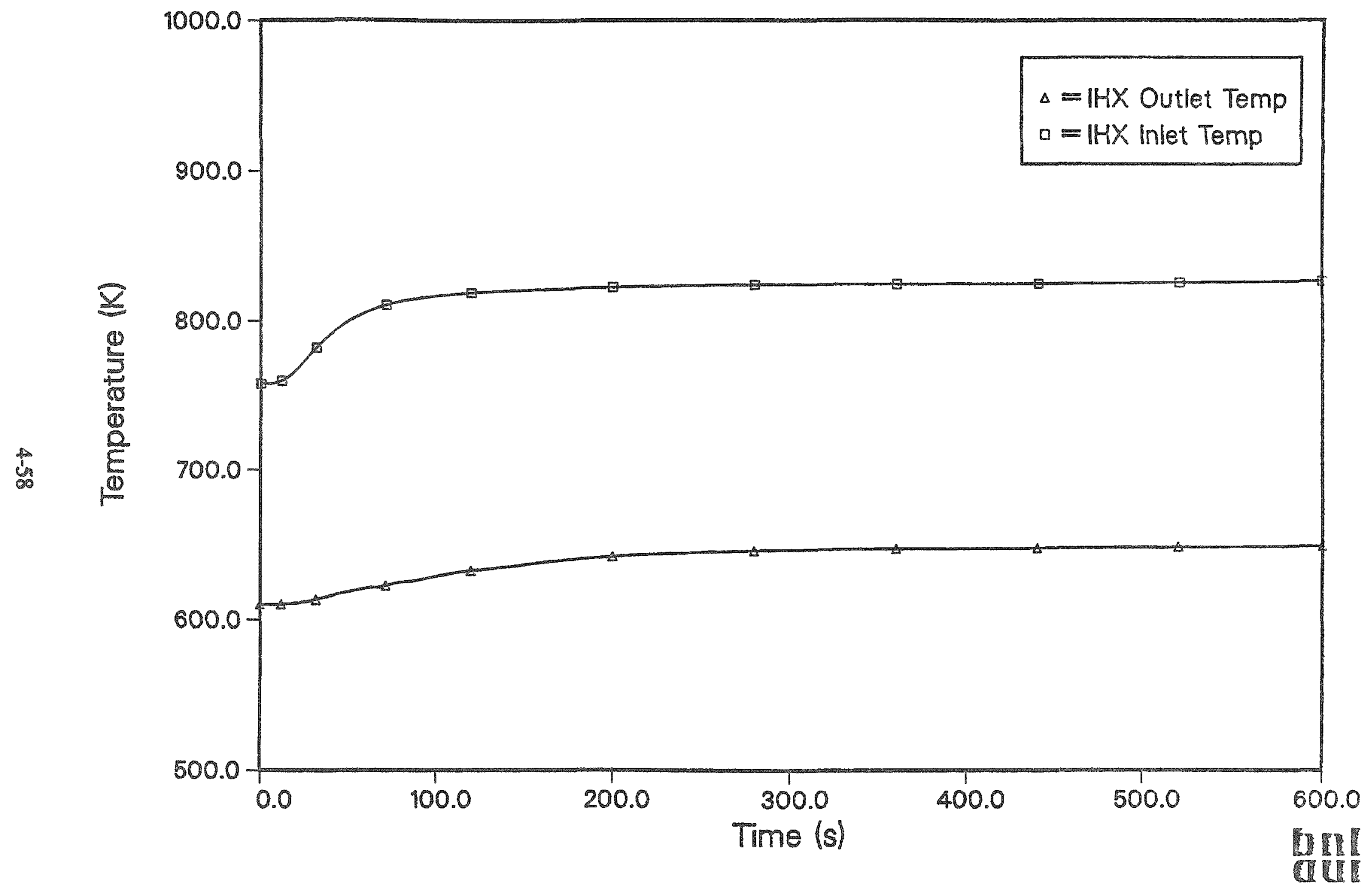

Figure 22. Predicted MIX Inlet and Outlet Temperature Predictions from SSC for PRISM During a 40 Cent UTOP for Assumptions Similar to ARIES. 


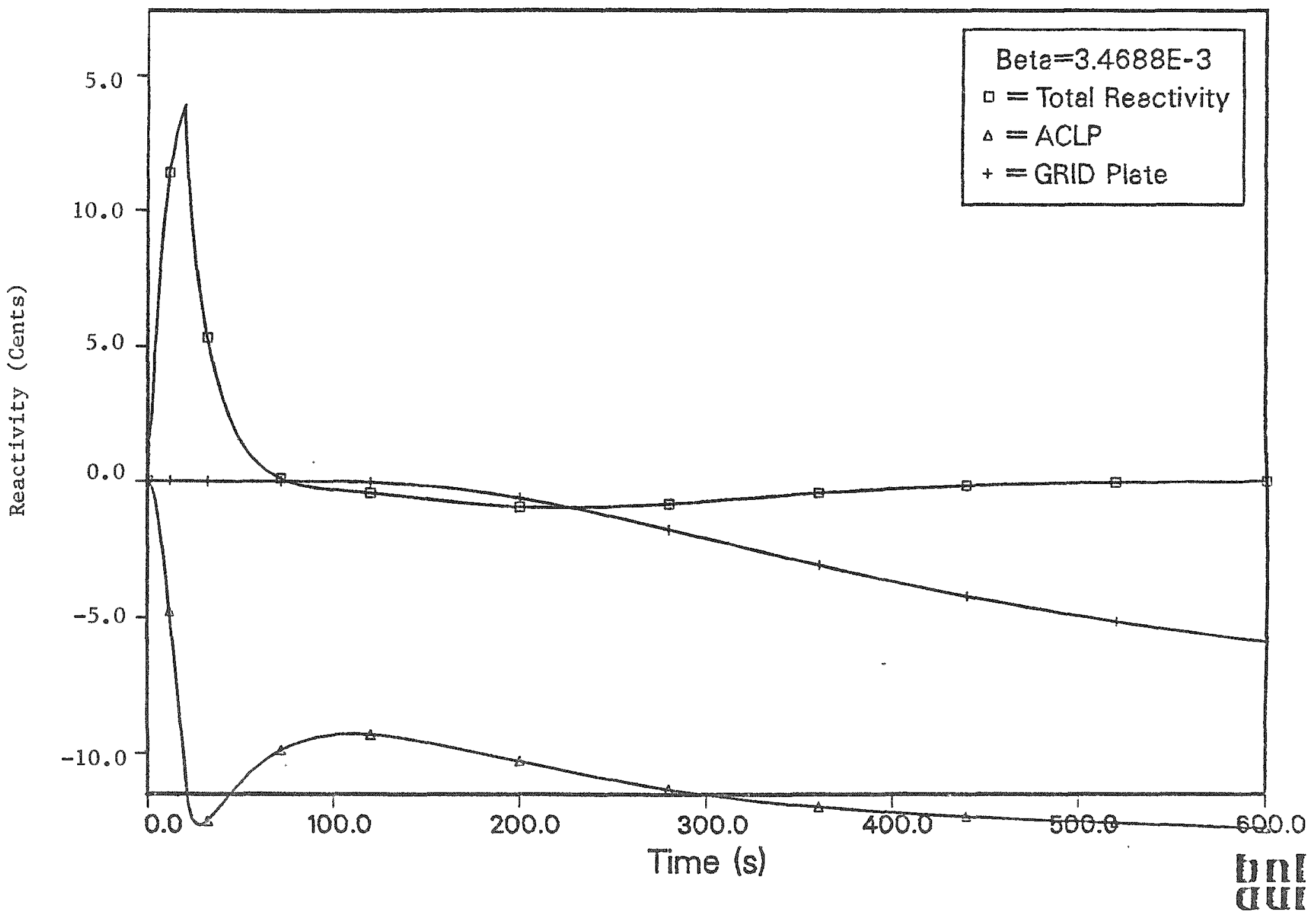

Figure 23. Reactivity Feedbacks predicted for the 40 Cent UTOP from SSC Using Conditions Similar to ARIES. 


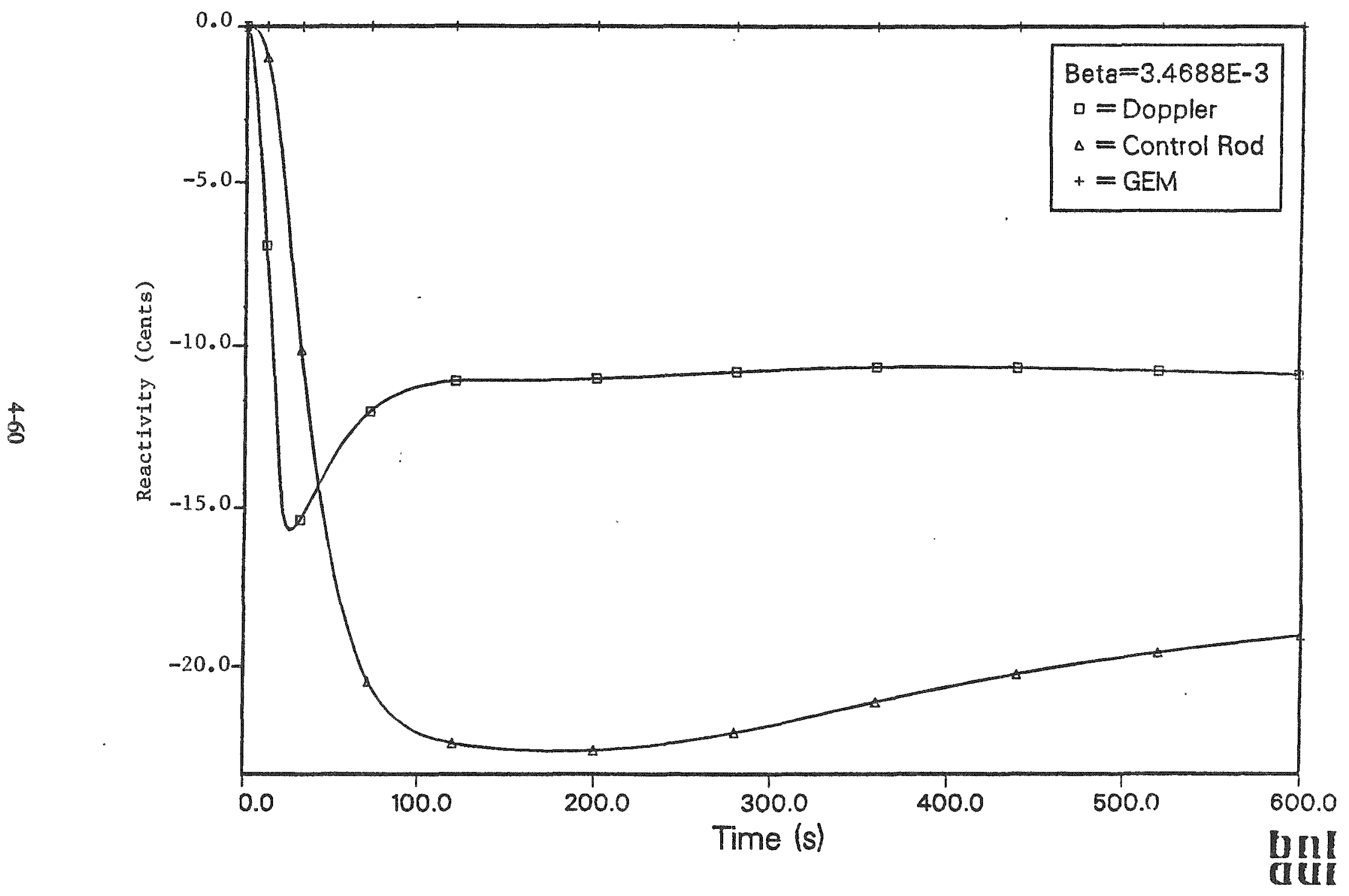

Figure 24. Reactivity Feedbacks Predicted for the 40 Cent UTOP from SSC Using Conditions Similar to ARIES. 


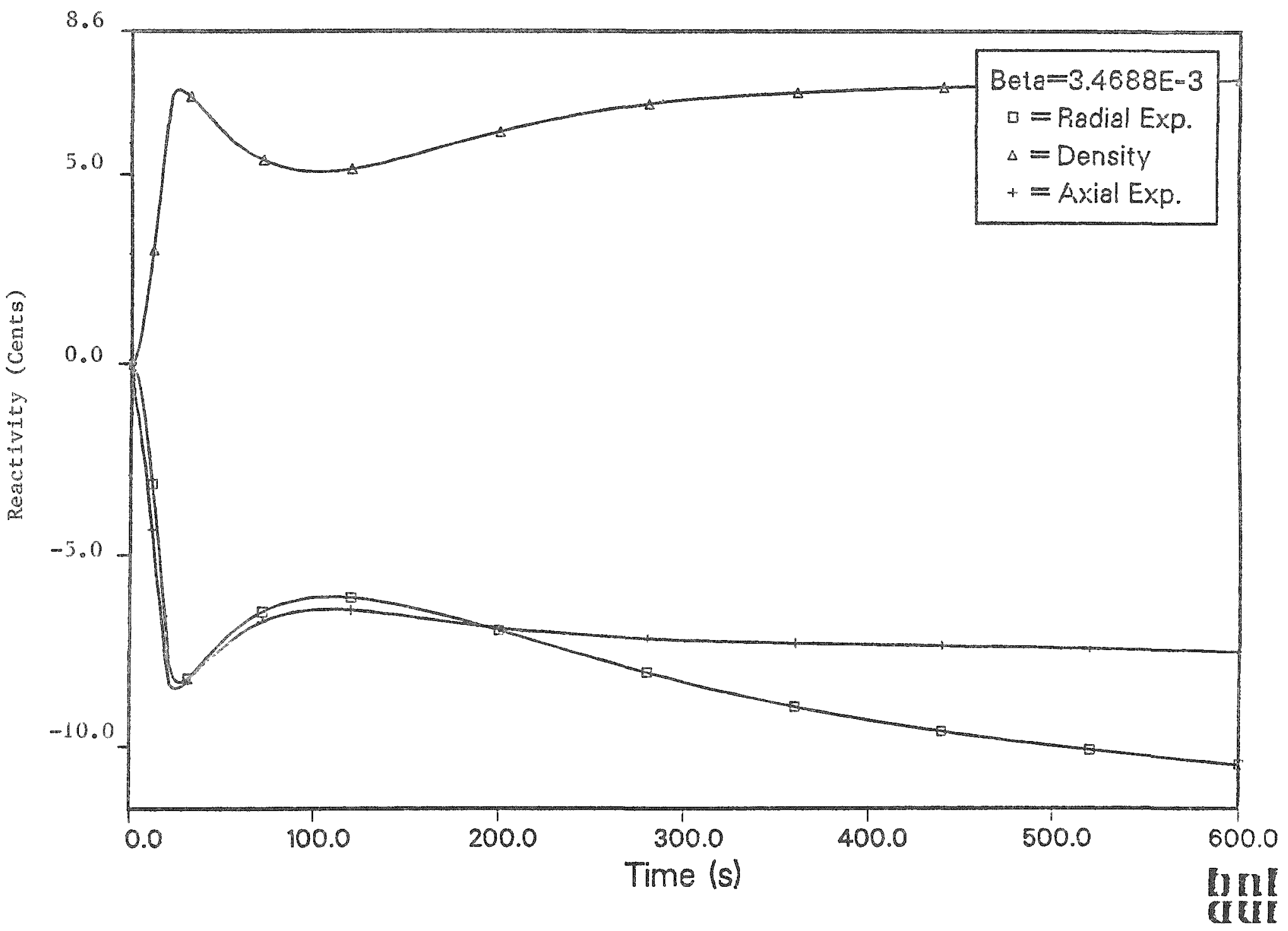

Figure 25. Reactivity Feedbacks Predicted for the 40 Cent UTOP from SSC Using Conditions Similar to ARIES. 


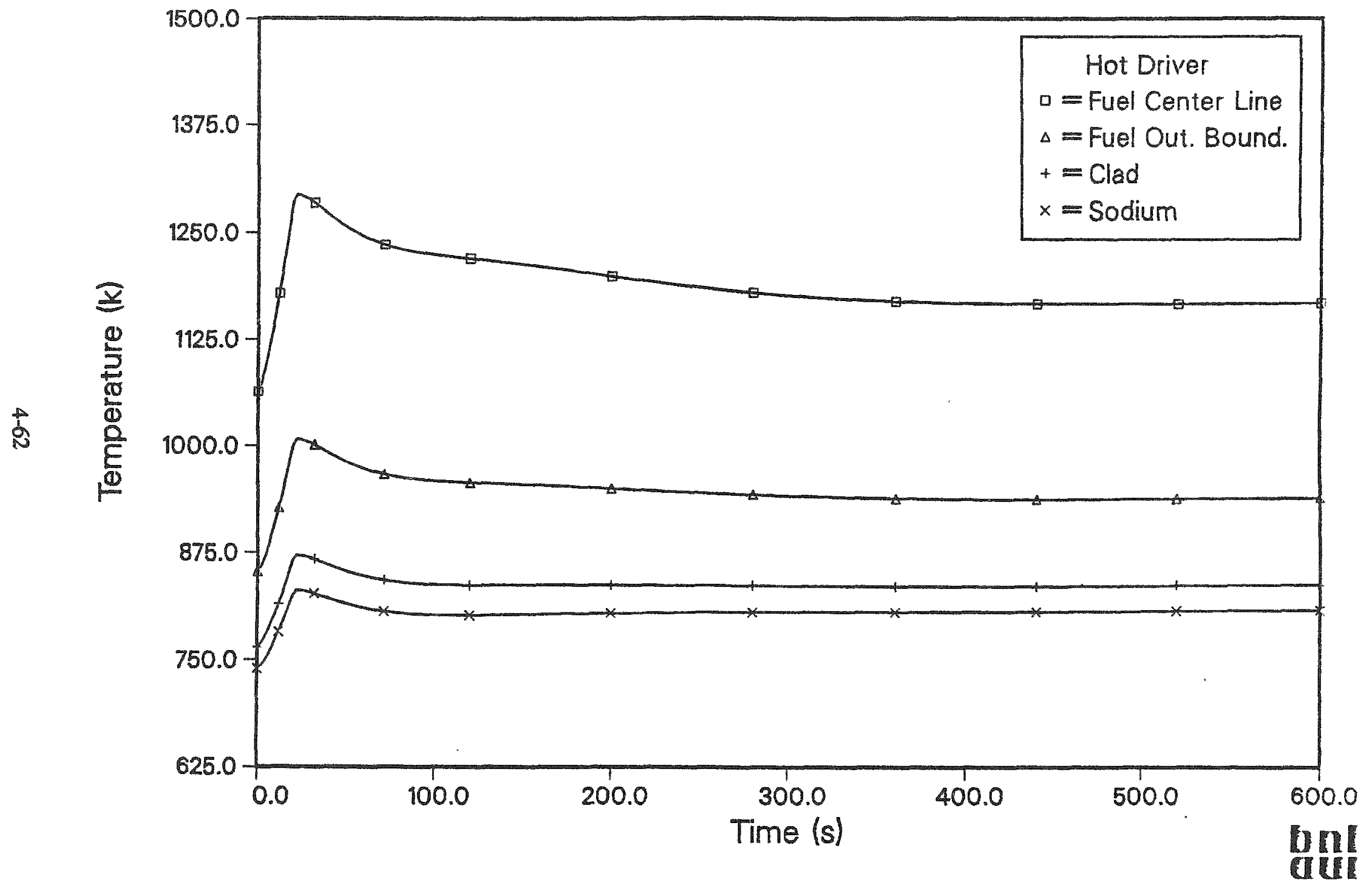

Figure 26. Predicted Fuel Temperature Distribution from SSC for the Third Node from the Top (i.e., $0.898 \mathrm{~m}-0.674 \mathrm{~m}$ ) Using Nominal Ternary Fuel Thermal Conductivities. 


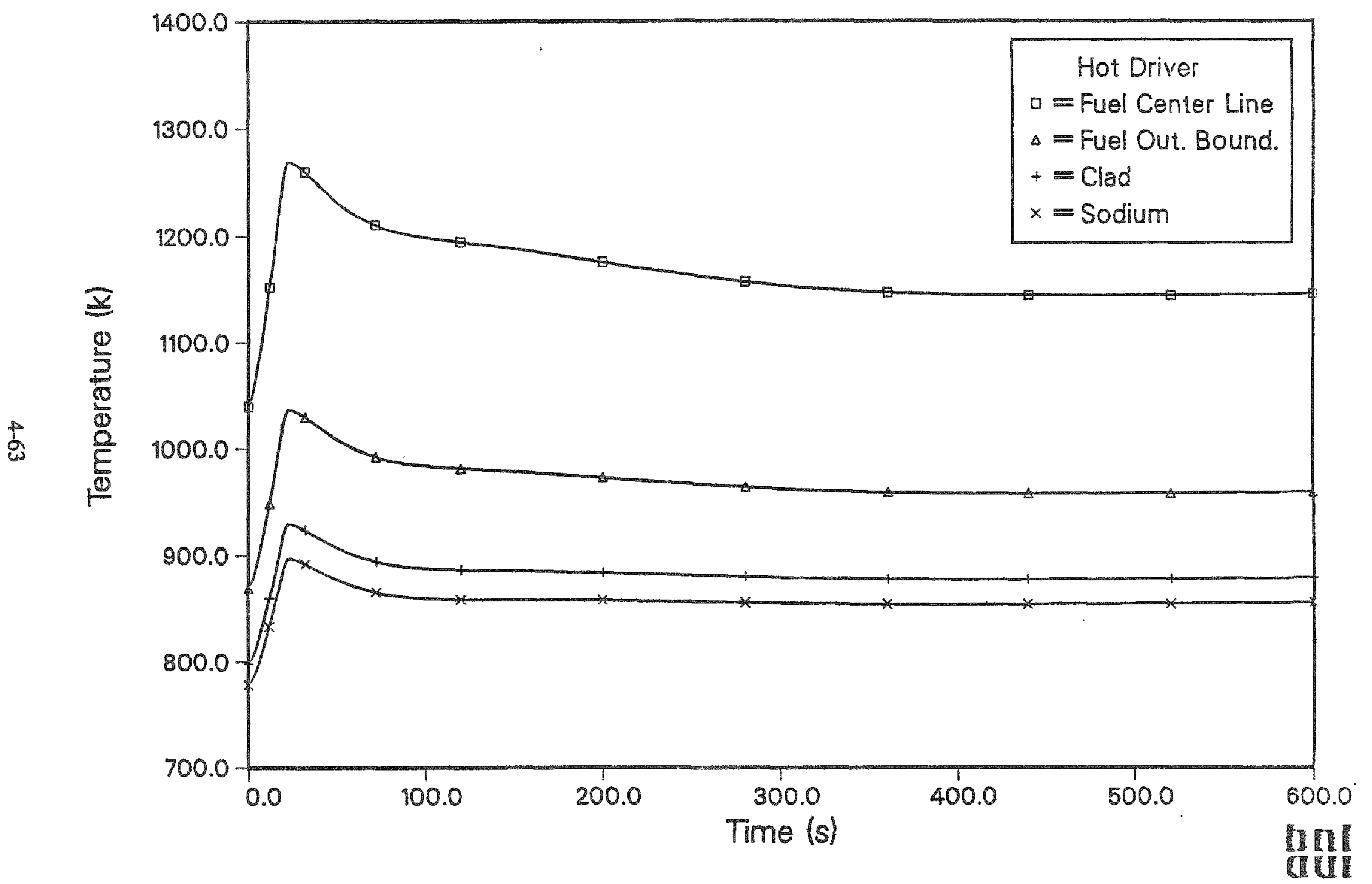

Figure 27. Predicted Fuel Temperatures from SSC for the Second Node from the Top (i.e., $1.122 \mathrm{~m}-0.897 \mathrm{~m}$ ) for a 40 Cent UTOP Using Nominal Ternary Fuel Thermal Conductivities. 


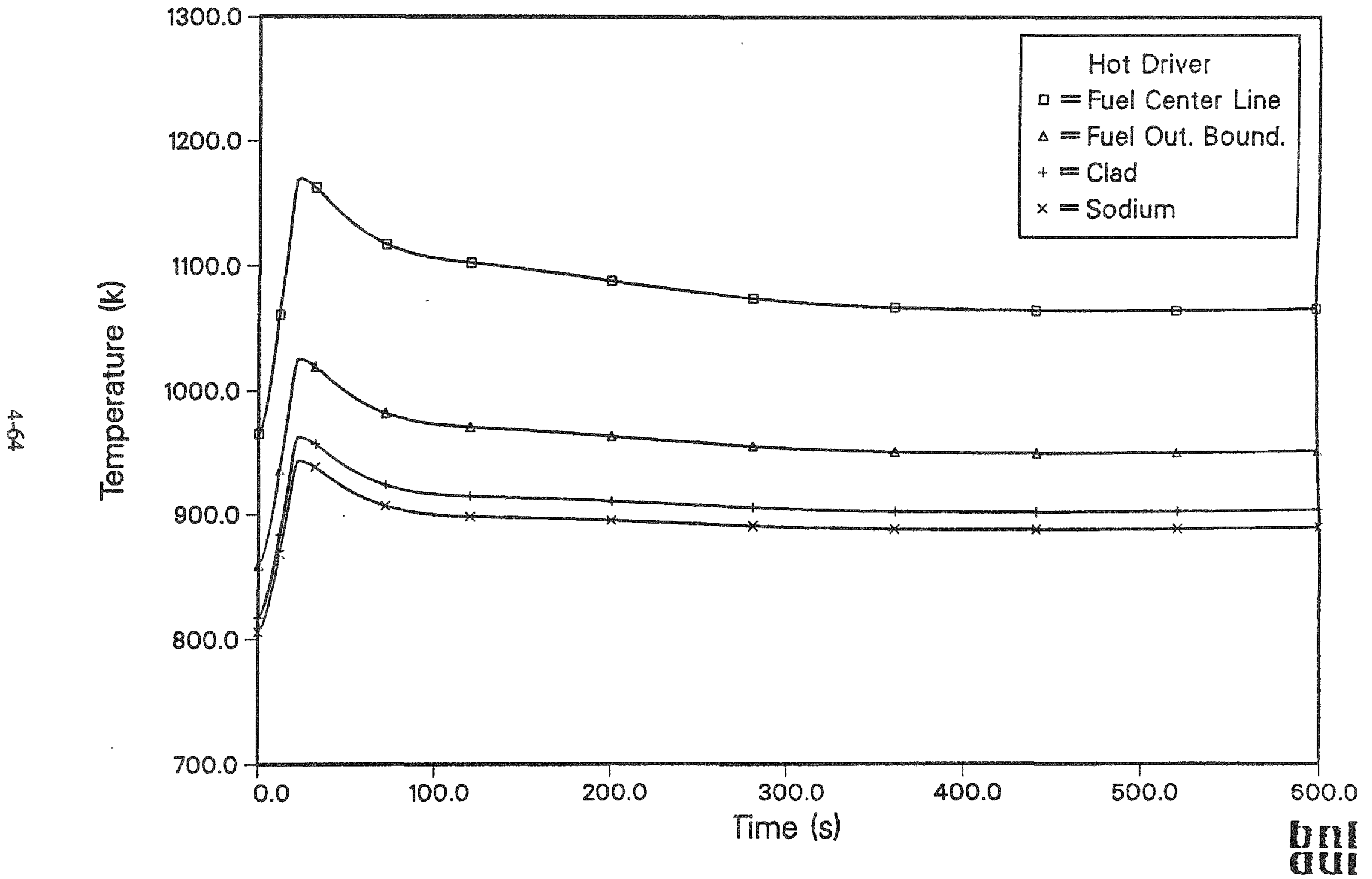

Figure 28. Predicted Fuel Temperature Distribution for the Top $0.224 \mathrm{~m}$ (i.c., $1.346 \mathrm{~m}-1.122 \mathrm{~m}$ ) of the Fuel Pin Using Nominal Ternary Fuel Thermal Conductivities During a 40 Cent UTOP. 
Table 2

Table of Peak Temperatures Predicted by SSC and ARIES

$\begin{array}{lcc}\text { Parameter } & \text { ARIES } & \underline{\text { SSC }} \\ \text { Peak Power } & 1.72 & 1.7 \\ \text { Peak Na (K) } & 951 & 944 \\ \text { Peak Clad (K) } & 979 & 960 \\ \text { Peak Fuel } & 1292 & 1298 \\ \text { Average Driver Peak (K) } & - & 1164 \\ \text { Na Saturation }(K) & - & 1340\end{array}$

conductivity is set to that of the nominal fuel, so that SSC could be consistent with the ARIES calculation. (This is changed in the next SSC calculation). The figures show that the peak temperatures are in the core center (axially) rather than near the top, as was the case in the previous PRISM design analyses. Excess reactivity, needed to compensate for the axial expansion reactivity loss and other factors, requires the control rods to be inserted deeper in the core than previously (also causing the need for the control rod stops), resulting in stronger bottom power peaking. The fuel centerline temperature plotted in Figure 26 shows that the temperature reaches $1298 \mathrm{~K}$, which is above the $1273 \mathrm{~K}$ solidus temperature for the nominal fuel. This solidus temperature is not thought to be conservative, since local melting temperatures can be impacted by isotope migration, which can reduce the solidus and liquidus temperatures.

Fuel melting was predicted in both the ARIES and SSC calculations. The extent of melting depends on the peaking factors used and the thermal properties. GE has estimated a peaking factor (i.e., peak driver pin compared to the average driver pin) of 1.31, which was used in both codes. However, it must be noted that this directly impacts the maximum temperature, and we do not have any direct confirmation that this peaking factor is appropriate. The thermal properties are still under review and have not yet been finalized for the present fuel in PRISM. The estimated behavior of the isotopes and their migration have not been resolved, either. Significant migration of the uranium and zirconium components occurs in the EBR-II fuel and is predicted for the PRISM fuel. High $\mathrm{Zr}$ levels reduce the solidus temperature and the thermal conductivity. Pu may also migrate, which could greatly reduce both the solidus temperature and the thermal conductivity, especially if the impact on local volumetric heating rates within the fuel is taken into account.

Because the metal fuel development program is ongoing, particularly with respect to the ternary fuel, several key factors have not yet been determined. The fuel is very dynamic when compared to oxide fuel, since the fuel experiences swelling, element migration, sodium logging, inter-porosity connections, fission product formation, and permanent axial expansion. The thermal conductivity is affected by all these factors, and irradiated fuel shows a minimum conductivity at $2 \%$ atom burnup when sodium logging (sodium filling the porosity within the fuel region) and inter-porosity connections have not been completed. The minimum conductivity is generally taken as $0.5 \pm 0.1$ times nominal, while 
the long term value is $0.7 \pm 0.1$, because of the sodium logging. It is believed that the fuel thermal conductivity is the least certain factor in the thermal hydraulic analysis for PRISM.

Conservative Case: UTOP with Reduced Thermal Conductivity

Two principal changes were made for this variant case. First, the control rod drive line was previously assumed to be "washed" by the sodium exiting from the surrounding channels in the lower part of the hot pool to be consistent with ARIES. This was changed to use the average sodium exiting the core, and the average of the hot pool, since local thermal hydraulic behavior during these events are not known. Further, the exact behavior of the flow around the UIS has not been determined. The second change was the reduction in the thermal conductivity for the hot channel, based on possible migration of the $\mathrm{Pu}$ and $\mathrm{Zr}$, and weighted by the volumetric heating rates. It is noted that plutonium migration may be insignificant, in comparison to the zirconium and uranium migration, but more data is needed before

one can conclude there is little or no $\mathrm{Pu}$ migration. Some of the resultant thermal conductivity values are shown in Table 3.

In Figures 29 through 37, results from the second, more conservative, UTOP calculation are represented. In Figure 29, the power peak is shown to increase to 1.8 times the rated power and later stabilizes at 1.2. The average core outlet sodium temperature reaches a peak of $880 \mathrm{~K}$. The reactivities in Figures 31, 32, and 33 show the same trends as before, except that the control rod drive line is slower to respond. In this more conservative analysis it is "washed" by the average sodium temperatures above the core rather than the hotter local exit temperatures immediately adjacent the control rod drive line. This slower response allows the peak power to reach 1.8 before the negative feedbacks start noticeably reducing the power level. It is noted that the sodium density feedback is the only active positive reactivity feedback (with temperature).

Table 3

Referenced Thermal Conductivities

Temp (K)

800

1000

1300
Nominal $(\mathrm{W} / \mathrm{mK})$

7.3

9.5

12.9
Reduced (W/mK)

2.5

4.6

9.8

The fuel temperatures for the top three nodes in the hot channel are shown in Figures 34, 35, and 36. The peak temperatures and power predicted during this calculation are shown in Table 4 . The peak center line temperature in Figure 34 reaches $1400 \mathrm{~K}$, which is far above the solidus temperature for the nominal fuel (i.e., $1273 \mathrm{~K}$ ), let alone for the case with the reduced solidus temperature from $\mathrm{Zr}$ migration, which is about $1200 \mathrm{~K}$ when $\mathrm{Zr}$ is about $2 \%$ atom percent in the center ring. Peak temperatures for the next axial level up in hot channel are shown in Figure 35, with the fuel temperature peaking near $1364 \mathrm{~K}$, which exceeds the solidus temperature for both nominal and $\mathrm{Zr}$ reduced fuel. However, the top node is much cooler, at $1258 \mathrm{~K}$, which indicates that prefailure extrusion, which projects molten fuel into the gas plenum from trapped molten pockets expelled by pressurized fission gas, will not occur, since the top-of-fuel temperatures are not hot enough (i.e., close to the solidus temperature). Thus, this expected negative feedback from nearly molten fuel would be unlikely to help mitigate this event. 


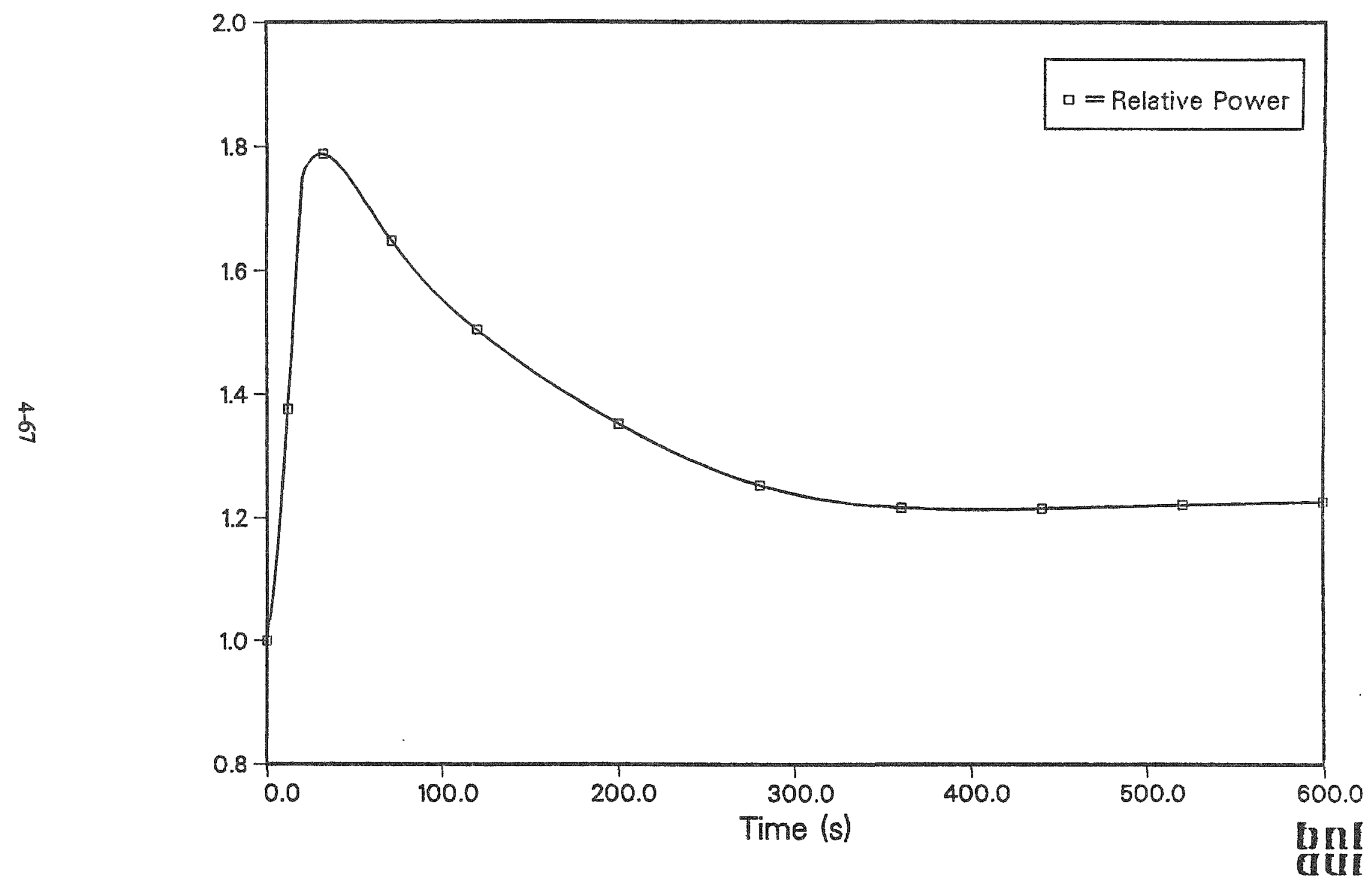

Figure 29. Relative Power Prediction from SSC for PRISM During a 40 cent UTOP. 


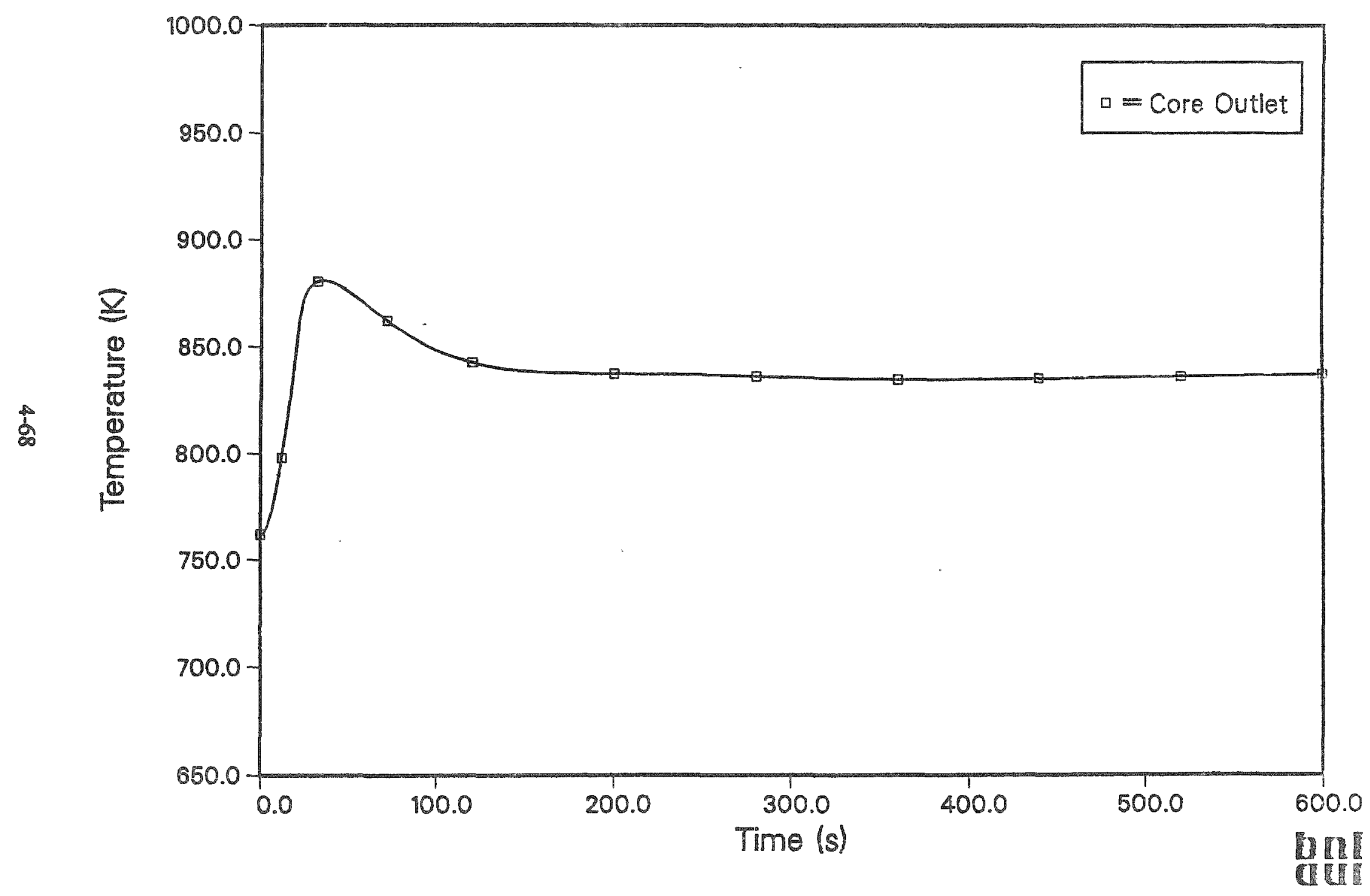

Figure 30. Average Core Outlet Temperature Predicted from SSC for PRISM During a 40 Cent UTOP. 


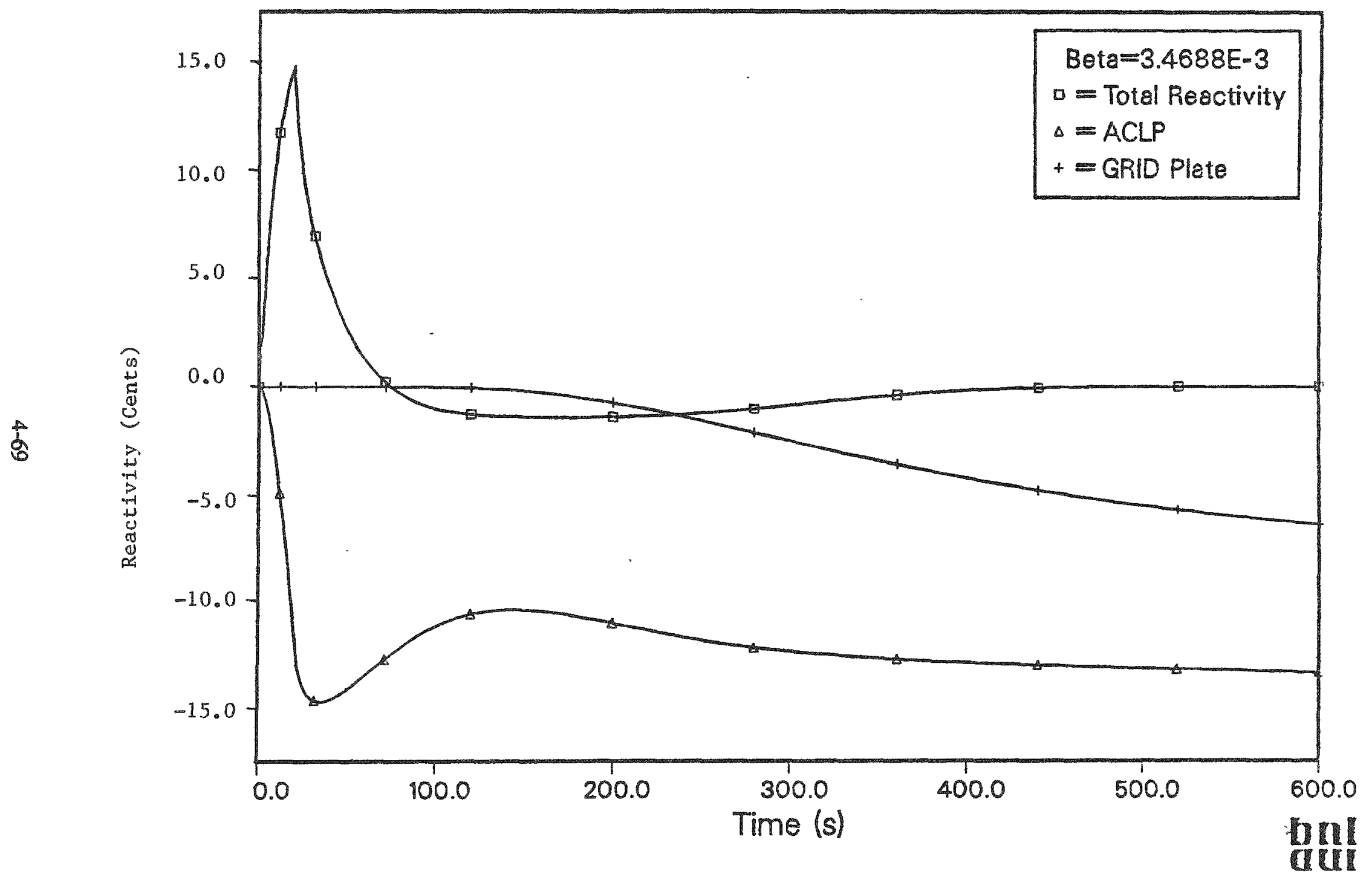

Figure 31. Predicted Total, Above Core Load Pad (ACLP) and Core Support Grid Plate Reactivity Feedback from SSC for a 40 Cent UTOP. 


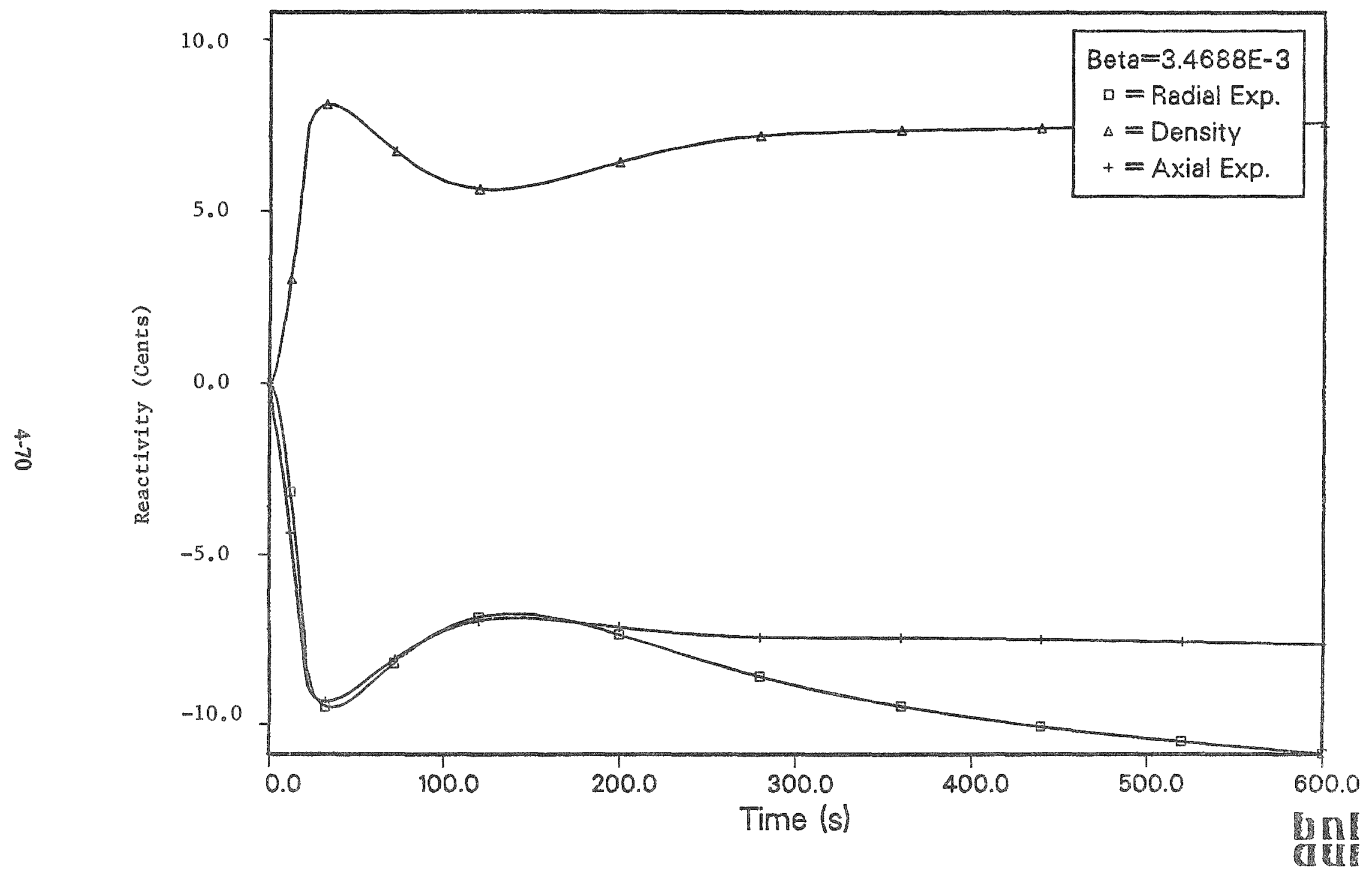

Figure 32. Predicted Radial Expansion, Sodium Density (Density), and Axial Expansion Reactivity Feedback from SSC for a 40 Cent UTOP. 


\section{$\min$}

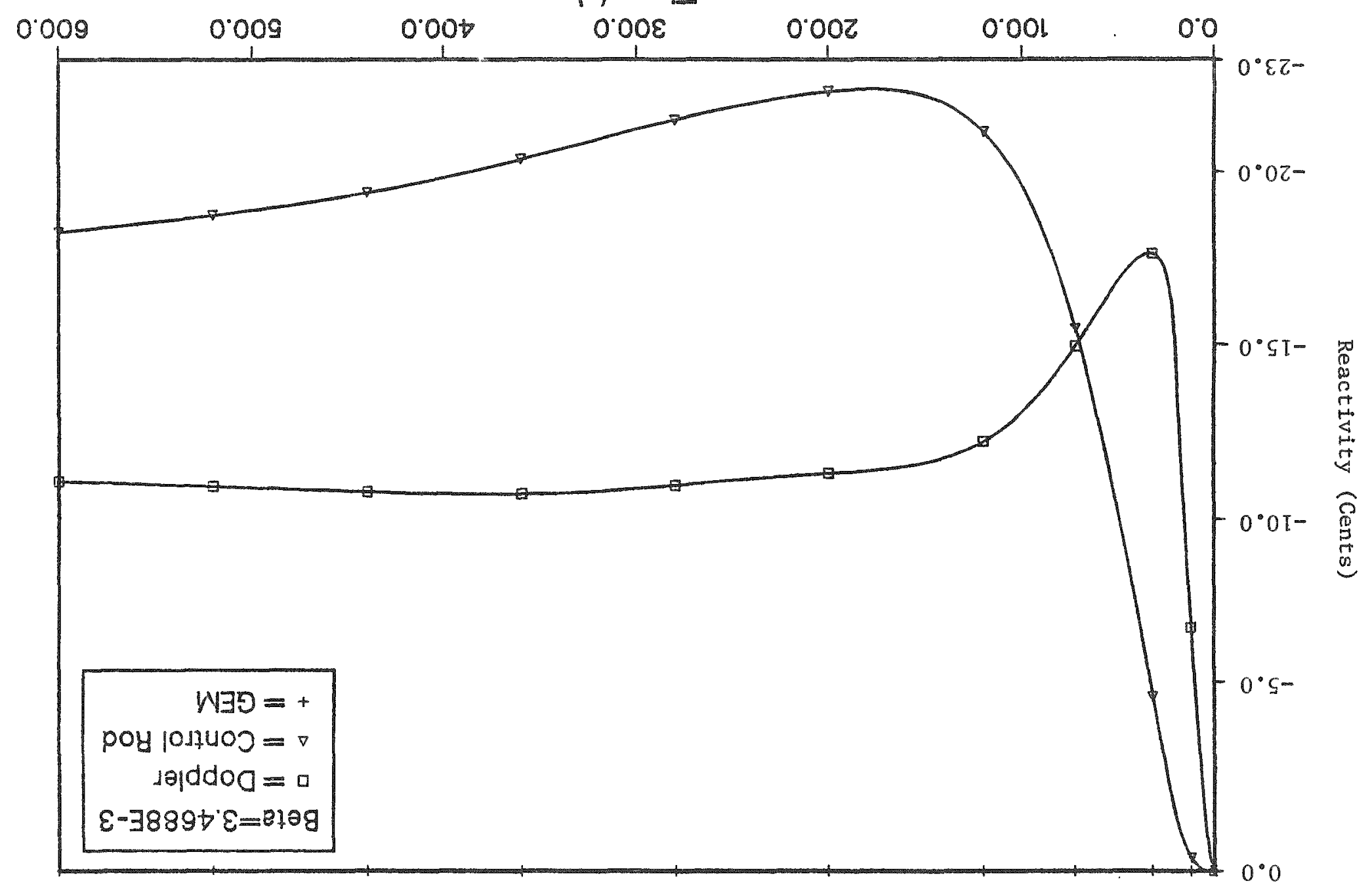




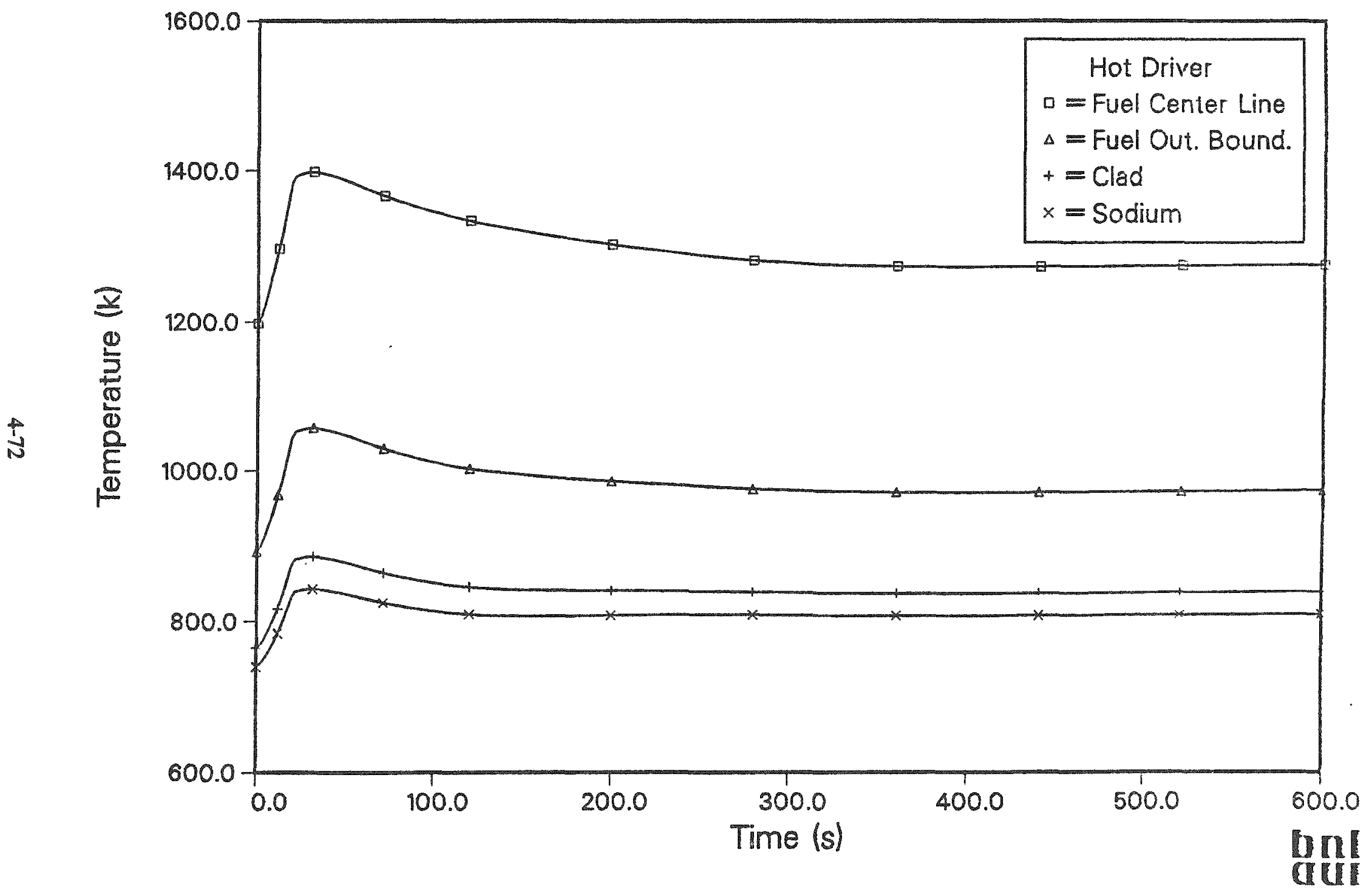

Figure 34. Predicted Fuel Temperature Distribution from SSC for the Third Node From the Top (i.e., $0.897 \mathrm{~m}-0.673 \mathrm{~m}$ ) During a 40 Cent UTOP. 


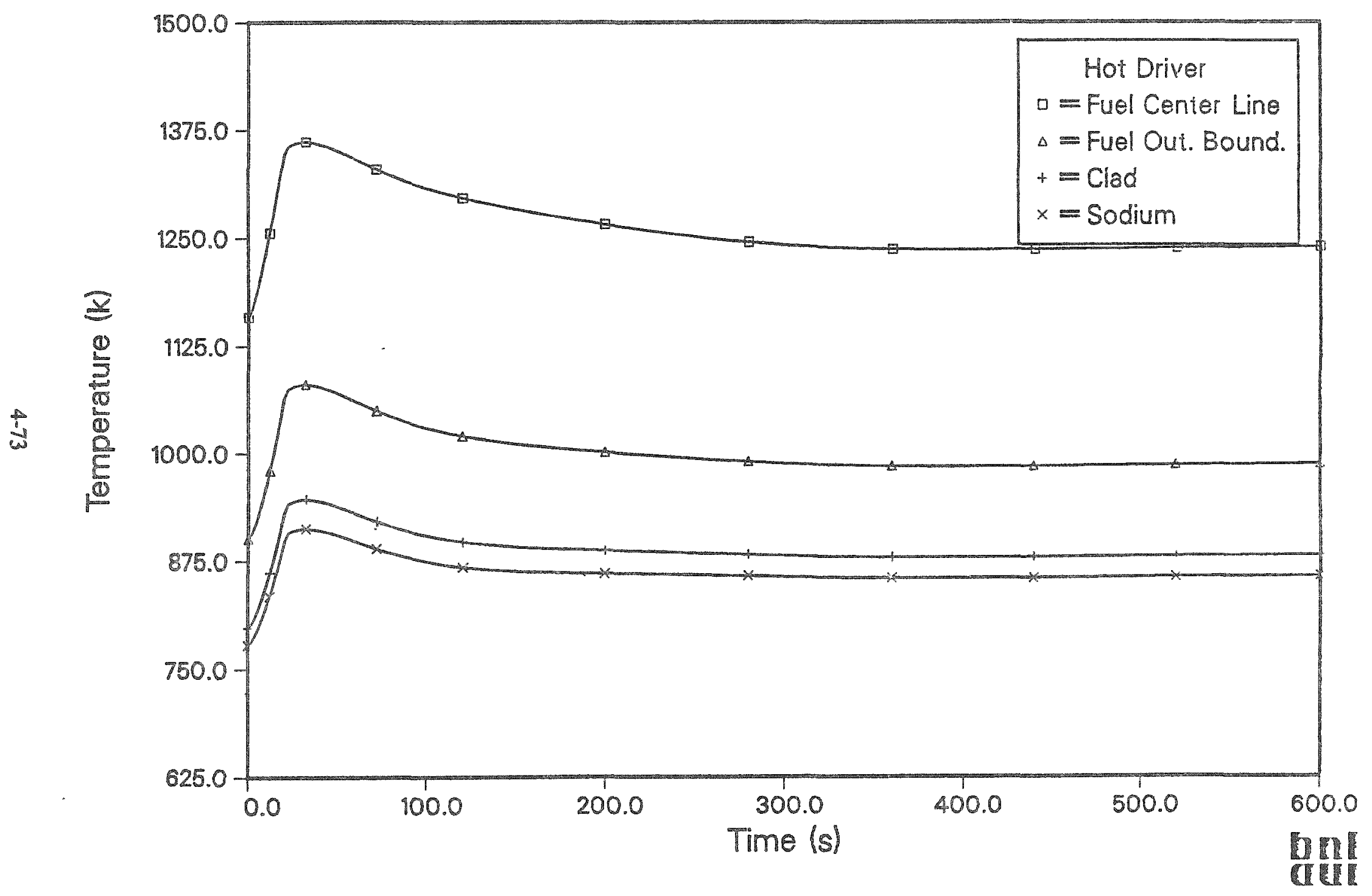

Figure 35. Predicted Fuel Temperature Distribution from SSC for the Second Node from the Top (i.e., $1.122 \mathrm{~m}-0.897 \mathrm{~m}$ ) During a 40 Cent UTOP. 


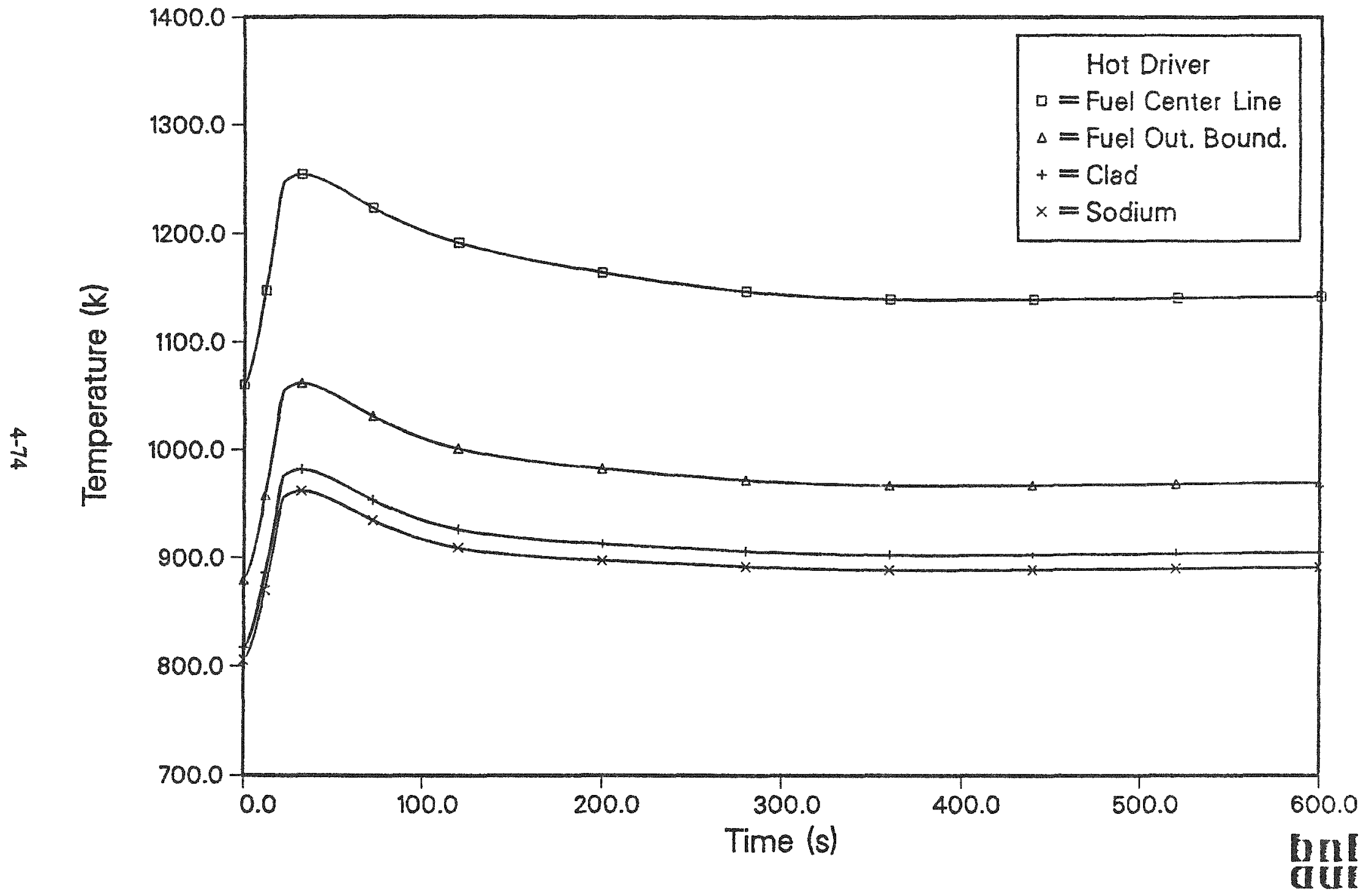

Figure 36. Predicted Fuel Temperature Distribution from SSC for the Top Node (i.e., $1.346 \mathrm{~m}-1.122 \mathrm{~m}$ ) 


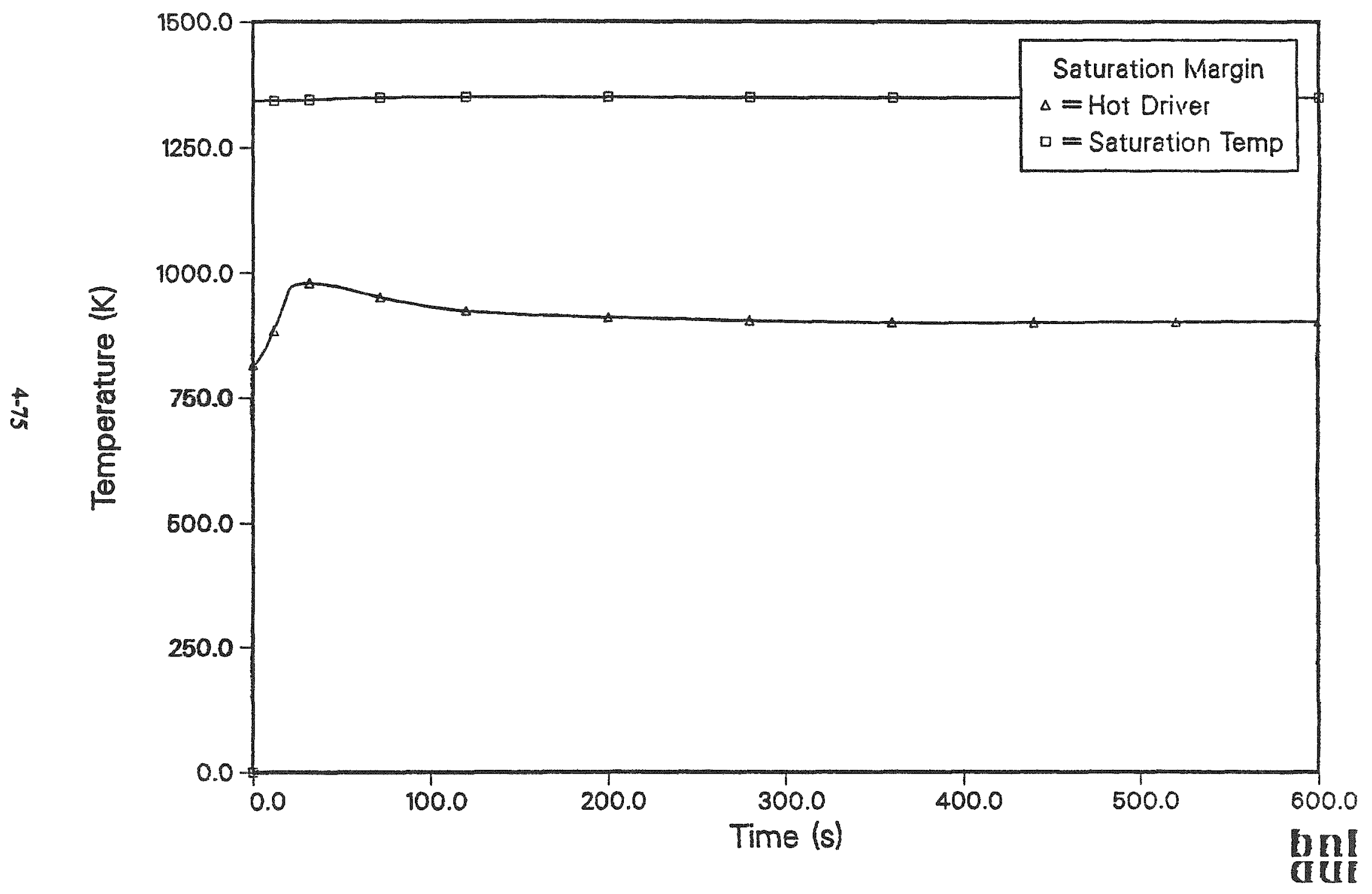

Figure 37. Predicted Sodium Saturation Margin from SSC for the 40 Cent UTOP. 
Table 4

Peak Values for UTOP Variant Case

$\begin{array}{ll}\text { Relative Power } & 1.8 \\ \text { Fuel Centerline }(K) & 1400.0 \\ \text { Sodium }(K) & 963.0 \\ \text { Clad }(\mathbf{K}) & 981.0 \\ \text { Average Driver }(K) & 1185.0\end{array}$

In summary, two SSC calculations were performed for the postulated UTOP event. The first used conditions and models similar to the ARIES code calculation to verify that the SSC analysis could be made to give results. The results indeed showed good agreement when both codes included similar modeling assumptions. The second SSC calculation for the UTOP event used the reduced thermal conductivity, to account for uncertainties and irradiation effects. Also, the average upper plenum pool temperatures were used to "wash" against the control rod drive lines, just above the core, as opposed to the flow from surrounding channels, as was used in the ARIES calculation.

All three calculations predict that some fuel melting will occur. When uncertainties in the thermal conductivity are included, the results suggest that much of the upper portion of the peak pin could experience localized melting.

While SSC does not represent phase changes or any other fuel melting phenomena, the high temperatures predicted indicate that the potential for significant melting is very real. These calculations were performed at the $2 \%$ burnup level, corresponding to the lowest value of thermal conductivity in the burnup cycle, and at that time the fuel would not have swollen out to the clad, except at the center (axial) location. Rather, the fuel-clad contact would occur according to the axial power shape, which would make the center close first, followed by the bottom, and finally the top of the fuel.

Some of these concerns have been discussed with GE and ANL. While both parties hope and/or believe that further data on the metal fuel behavior will reduce the concerns, GE has provided a brief list of changes that could be made to the PRISM design in order to alleviate our concerns. These options are as follows:

1) Moving the control rod stops more frequently and by smaller increments

2) Making multiple rod withdrawals extremely improbable

3) Increasing B-10 enrichment in control rods so driveline expansion is more effective

4) Changing pin characteristics

5) Reducing plutonium content

While each of these steps may have strengths and shortcomings, it is clear that GE has several options. Therefore, while the current results of the 40C UTOP indicate fuel damage, it is clear that the applicant has several options to reduce or eliminate this apparent vulnerability. 


\subsection{B.E. 1B: UTOP with Only RVACS Cooling}

In order to analyze this lengthy unscrammed event, we needed to use the long term heat-up calculation from MINET coupled with a long term reactivity transient analysis from SSC. This capability is planned as part of the Advanced LMR Transient Analysis Package (ALTAP), which is to be created from SSC, MINET, and a couple of smaller special purpose computer models.

While we could not fully simulate this event, we can make some judgements based on GE's calculations and our own independent analyses for related cases. First, our calculations for the long term heat-up events (MINET) are very similar to those provided by GE. This is true for the reference RVACS heat-up event, which is the scrammed version of the event considered here. Therefore, we can assume that the rate of heat removal in the $G E$ analysis of Event $1 B$ is reasonably accurate. Second, with the exception of GE's misrepresentation of the reactivity worth of control rod motion (they inadvertently used the reactivity worth curve for the previous PRISM reactor design), GE's results for the unscrammed events are in good agreement with our SSC calculations (when we assume nominal fuel conditions consistent with GE's calculations). Therefore, we would expect GE's analysis of Event $1 \mathrm{~B}$ to under predict the reactor power and temperatures, but by a relatively small amount. Third, we can crosscompare the results from GE's two calculations for Events $1 \mathrm{~A}$ and $1 \mathrm{~B}$, as summarized in Figure 16. It is noted that the peak cladding and sodium temperatures are significantly higher for Event $1 \mathrm{~B}$, as the systems temperatures have to be considerably higher than in Event 1A for the RVACS to be able to effectively dump the heat. However, when the sodium and cladding temperatures are much higher, the power production must fall significantly. This is indicated by the fact that the peak fuel temperature in Event $1 \mathrm{~B}$ is not much higher than that in Event $1 \mathrm{~A}$.

Thus, we have several good reasons to believe GE's results are fairly accurate, with the two qualifications. First, the predicted powers and temperatures will be slightly under predicted by GE, due to the control rod modelling error. Second, GE's results neglect any plutonium migration, which cannot be ruled out with the present data base. Should future data indicate the plutonium is relocating, then the predicted peak temperatures will be significantly higher and the possible fuel damage could be quite serious.

Either way, we must conclude that the results for Event $1 \mathbf{B}$ are a reason for concern, since significant fuel melting is likely. However, we do not believe that Event $1 \mathrm{~B}$ is a major problem for the designer, as there are several options available to remedy this problem, with the most obvious being to simply move the control rod stops more frequently (see also Section 4.16.5). Therefore, while the analysis of Event $1 \mathrm{~B}$ indicates a potential safety problem, the fact is that the applicant has options to resolve the problem fairly easily with appropriate design changes.

\subsection{Variation on B.E.1: UTOP with LOF}

Although not specified as one of the postulated Bounding Events, the UTOP with the EM pumps tripped simultaneously is an interesting, although unlikely, event. This event was difficult to accommodate passively with the previous PRISM design, but the addition of the GEMs has had a major impact on the predicted response for this event.

The initial conditions are the same as the previous cases, as specified in Table 1. The transient begins with the simultaneous trip of the pumps and the withdrawal of the control rods. The four EM pumps coast down. The control rod withdrawal is worth 2 cents/s for 20 seconds, for a total of 40 cents. The secondary loop and IHX remain operational during this event. 
The decrease in flow and increase in reactivity causes a fast heat-up of the system. In Figures 38 and 39 , the predicted power and core outlet sodium temperature, respectively, are shown. At about $300 \mathrm{~s}$, the power and flow begins to stabilize, and natural circulation is established. Also, lower temperature sodium reaches the core, from the operating IHX, and decreases the reactor outlet sodium temperature.

The reasons for the decrease in power can be seen in Figures 40 through 42 , where the reactivity feedbacks are plotted. This initial response comes mostly from the 3 GEMs, as shown in Figure 42. As the pumps coast down, the sodium level in the GEMs drops, adding negative reactivity as the pump outlet pressure decreases. This holds down the power to flow ratio, so enough coolant flow is available to remove the heat generated. The fuel and channel ducts increase in temperature, expanding, and adding negative reactivity from radial expansion, as shown in Figure 41. However, the GEMs dominate the other feedbacks, causing the power to decrease. The power level settles around $10 \%$ of rated power, with the feedbacks from the GEMs and the temperatures of the structures having reached a new (critical) equilibrium point.

The reactor outlet sodium temperatures are shown in Figure 43, with the hot channel outlet peak predicted to be $1015 \mathrm{~K}$. The corresponding fuel temperature is the initial value, and thus is near the center of the core. The various temperatures in the hot driver channel, near the core center and the core outlet, are shown in Figures 44 and 45, respectively. The internal and radial blanket temperatures increase from the normal operating levels, but no fuel temperature limits are even approached. Figure 46 shows that the margin to boiling in the ULOF/TOP is $215 \mathrm{~K}$. The sodium level in the GEMs, which drops quickly as the pumps coast down, is shown in Figure 47. With the addition of the GEMs, it is now apparent that the PRISM design could withstand a ULOF/TOP of 40 cents. The power would transition to about $10 \%$ of the rated level, due to the negative reactivity feedbacks from the GEMs. No fuel damage is predicted.

\subsection{Peak UTOP Temperatures}

The peak temperatures predicted in the UTOP event calculations are shown in Figure 48, which also shows the pertinent temperature limits. As was discussed, the peak temperatures for the Event $1 \mathrm{~A}$ 40 C UTOP with the normal cooling were very similar, as long as we used GE's assumptions. Thus, the two sets of bars in the left half of Figure 48 are quite similar. Both calculations indicate some fuel melting in the hottest part of the core, although the damage would be pretty localized if nominal fuel is assumed. If zirconium migration is considered, the fuel damage would be more extensive. However, if the peak cladding and sodium temperatures appear to be acceptable, PRISM could probably survive the fuel melting if there was little or no effective compaction of the fuel material in the pins (a compaction would lead to increased reactivity and power production). However, if the lower cladding damage limit of $903 \mathrm{~K}$ (Section 4.16.3.2) applies, then significant cladding damage could occur.

Peak temperatures for the same event, if we assume plutonium migration, are indicated by the third set of bars in Figure 48 . The peak fuel temperatures are very high, although the peak clad and sodium temperatures are little changed. Even if the cladding remains intact, it appears that the fuel damage would be wide-spread and severe, assuming that plutonium migration is a genuine problem in the ternary fuel. Should further experiments indicate significant plutonium relocation, it may be necessary for GE to reduce the maximum credible TOP initiator.

The final case in Figure 48 is an instance where the design changes have resulted in enhanced safety, as this was previously one of the more hazardous unscrammed events. However, the addition of the GEMs has significantly improved PRISM response, and the principal concern remaining would be 


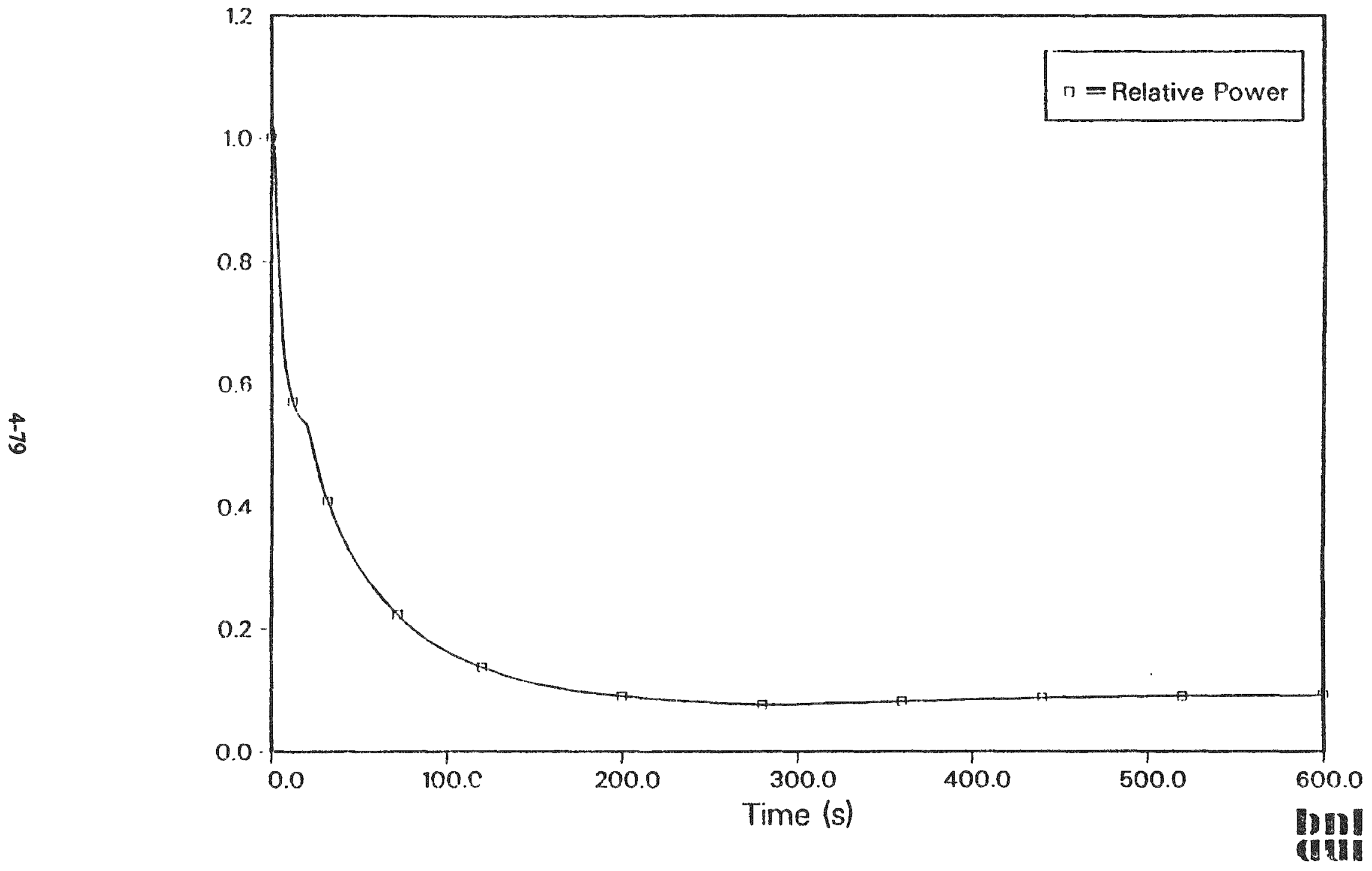

Figure 38. Relative Power Prediction from SSC for PRISM During a 40 cent UTOP/LOF. 


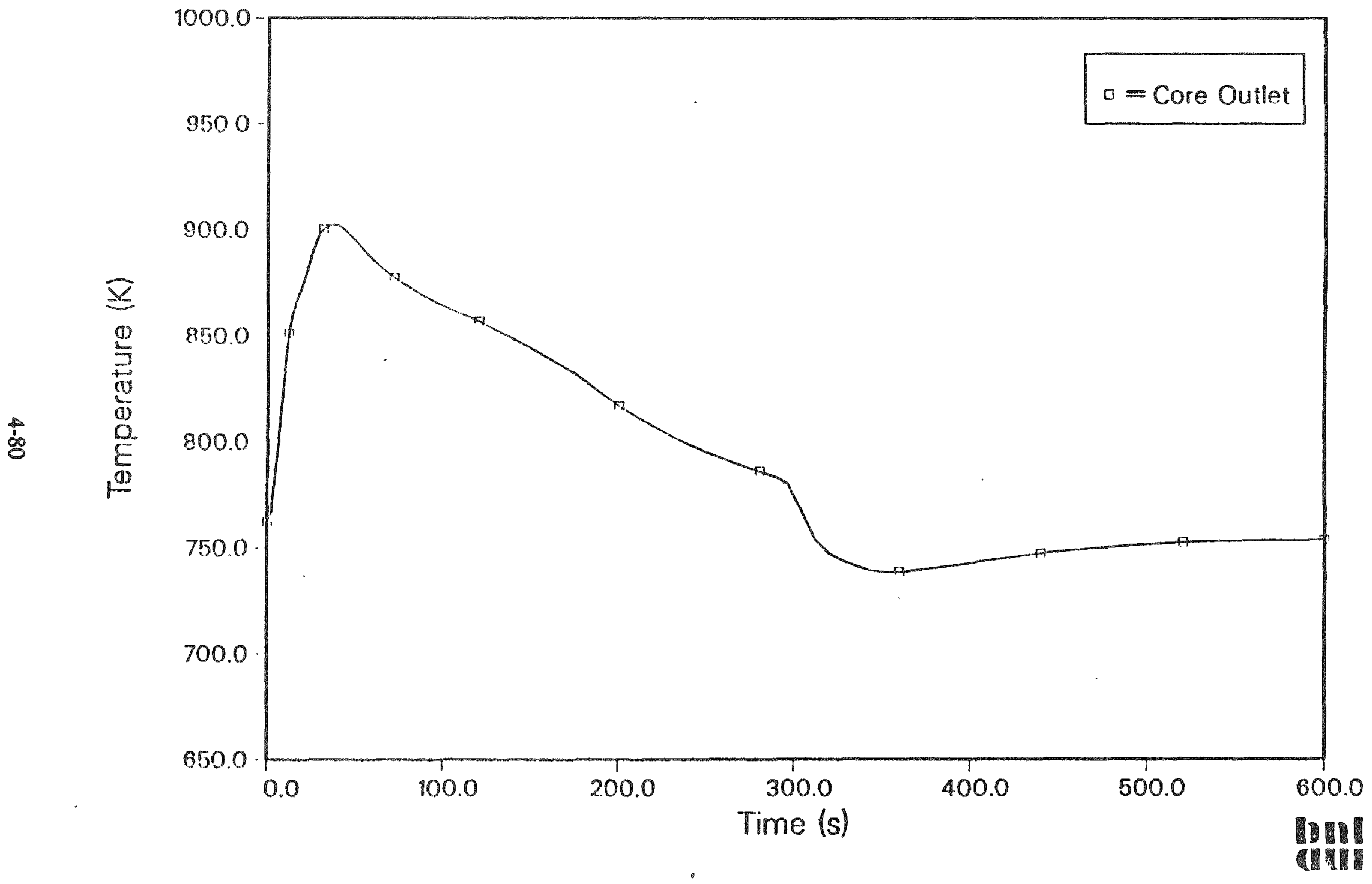

Figure 39. Average Core Outlet Temperature Prediction from SSC During a 40 Cent UTOP/LOF. 


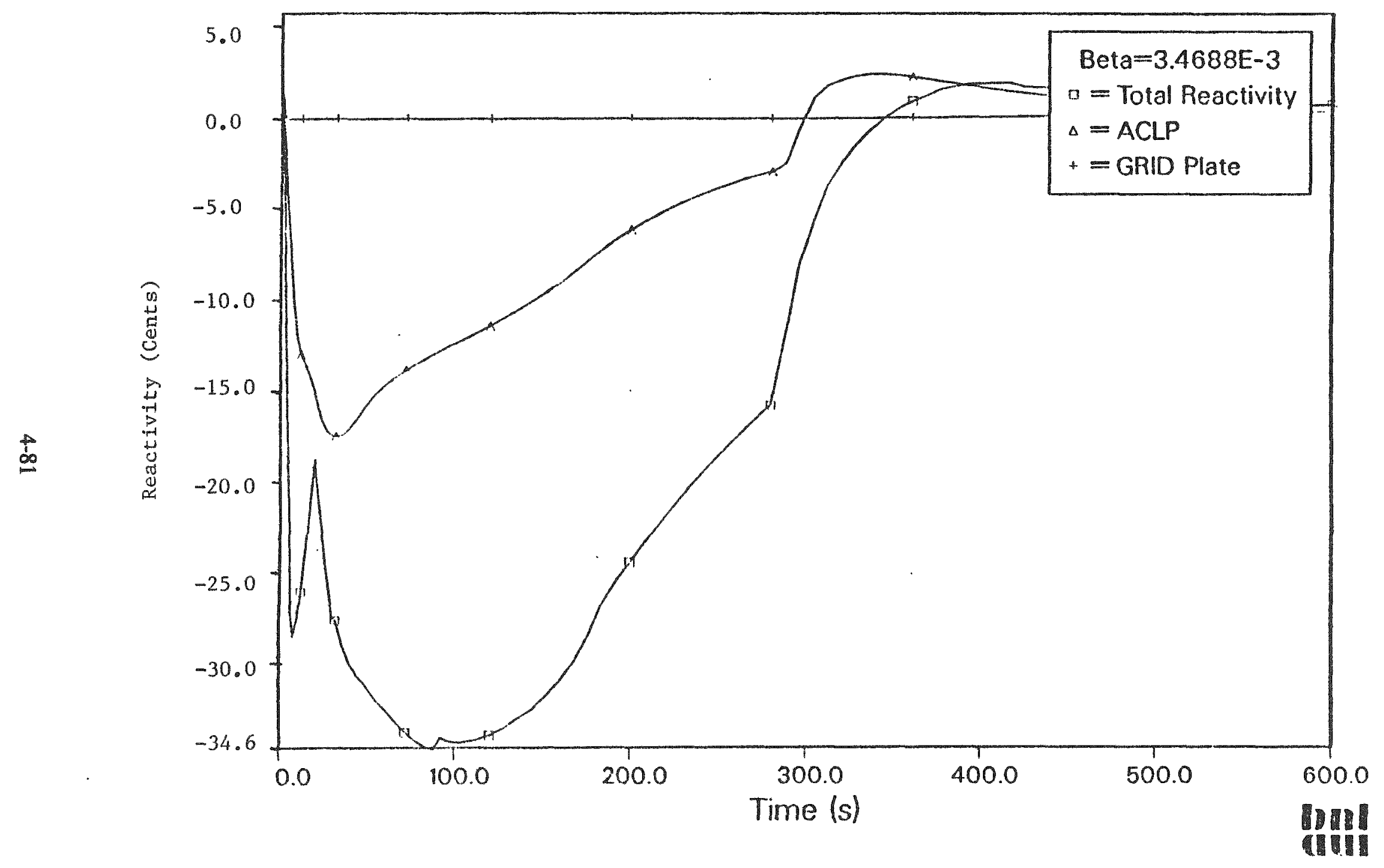

Figure 40. Predicted Total, Above Core Load Pad (ACLP) and Core Support Grid Plate Reactivity Feedback from SSC for a 40 Cent UTOP/LOF. 


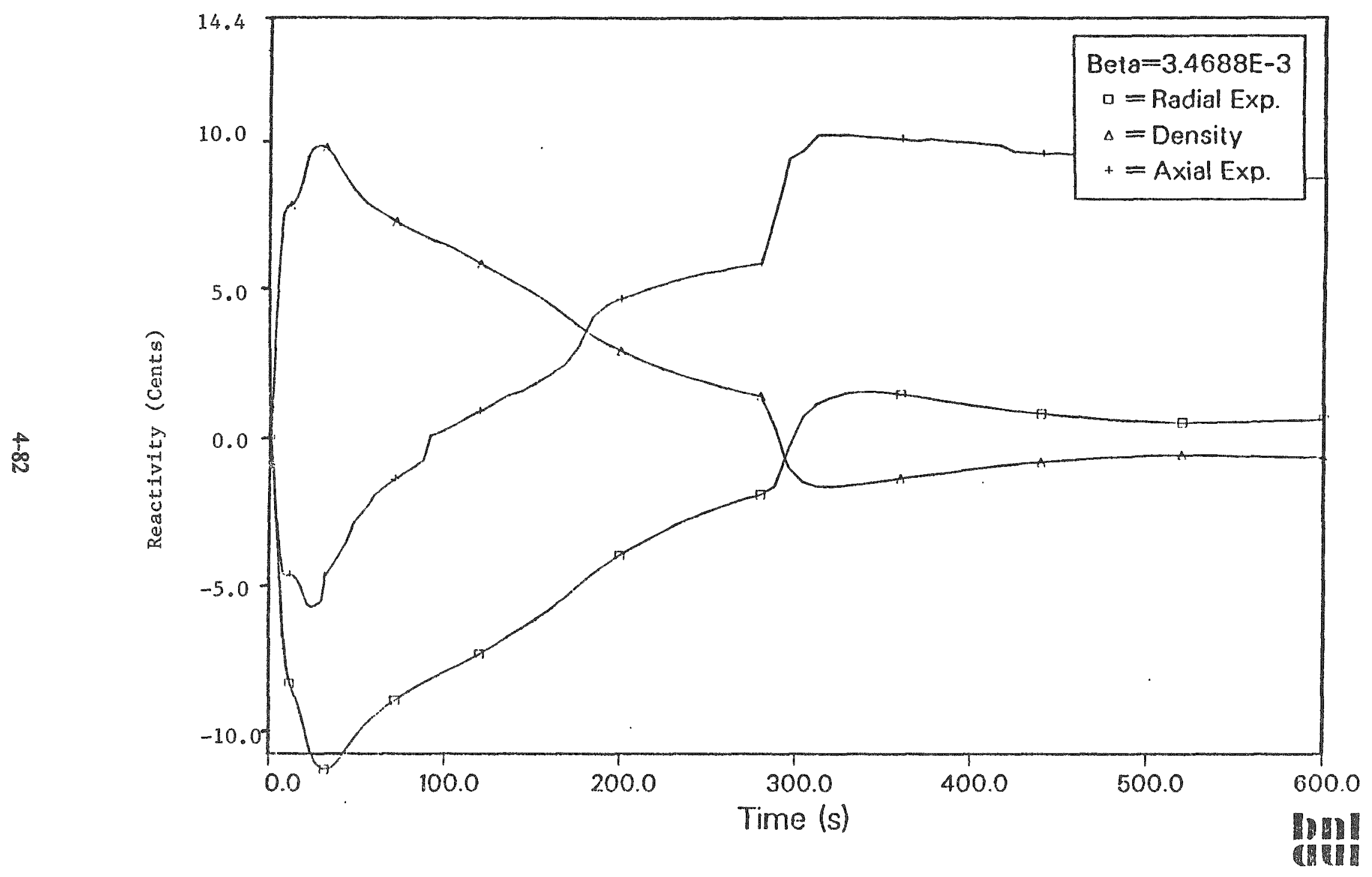

Figure 41. Predicted Radial Expansion, Sodium Density (Density), and Axial Expansion Reactivity Feedback from SSC during a 40 Cent UTOP/LOF. 


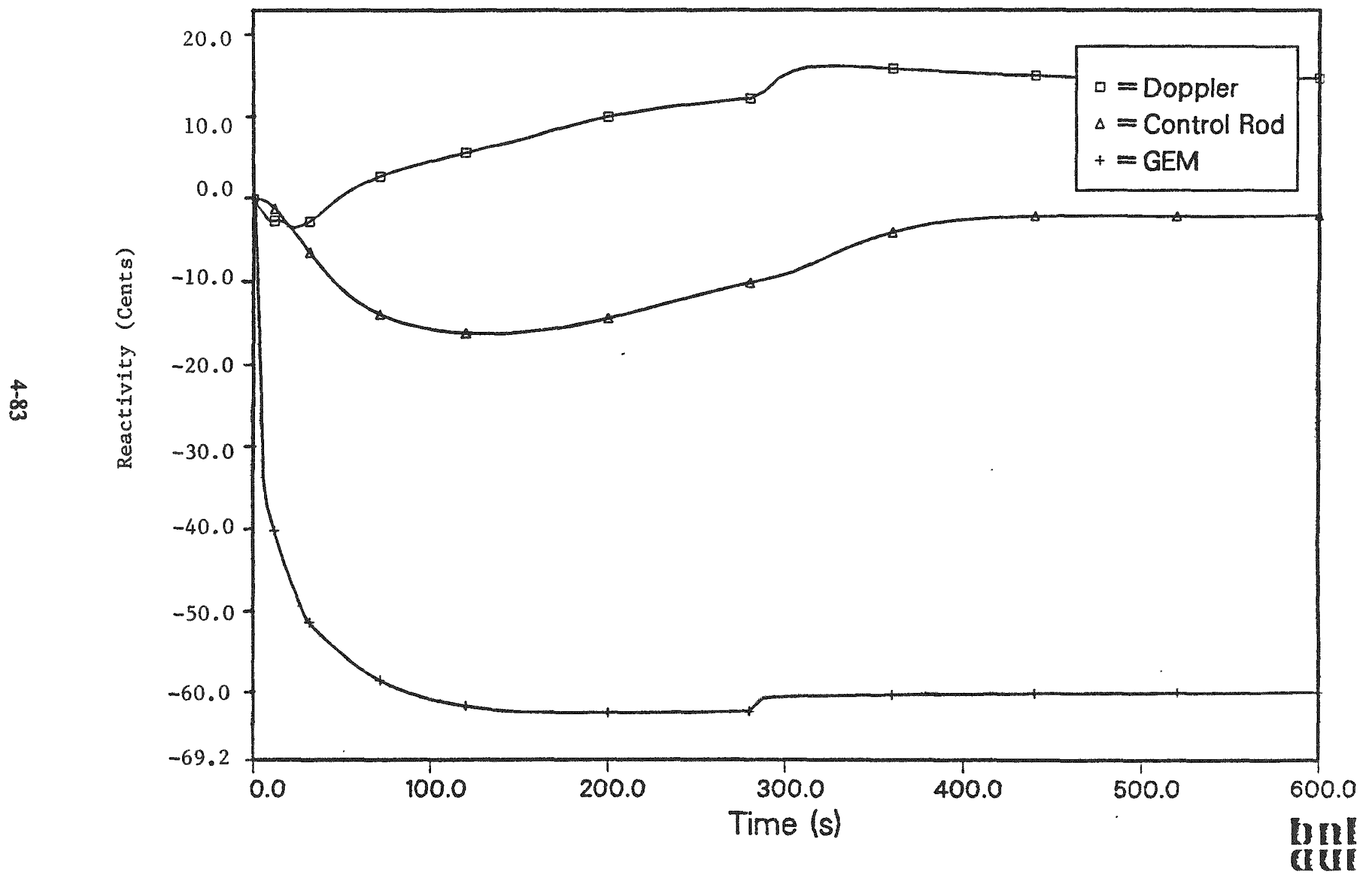

Figure 42. Predicted Doppler, Control Rod Expansion, and Gas Expansion Module (GEM) Reactivity Feedback from SSC during a 40 Cent UTOP/LOF. 


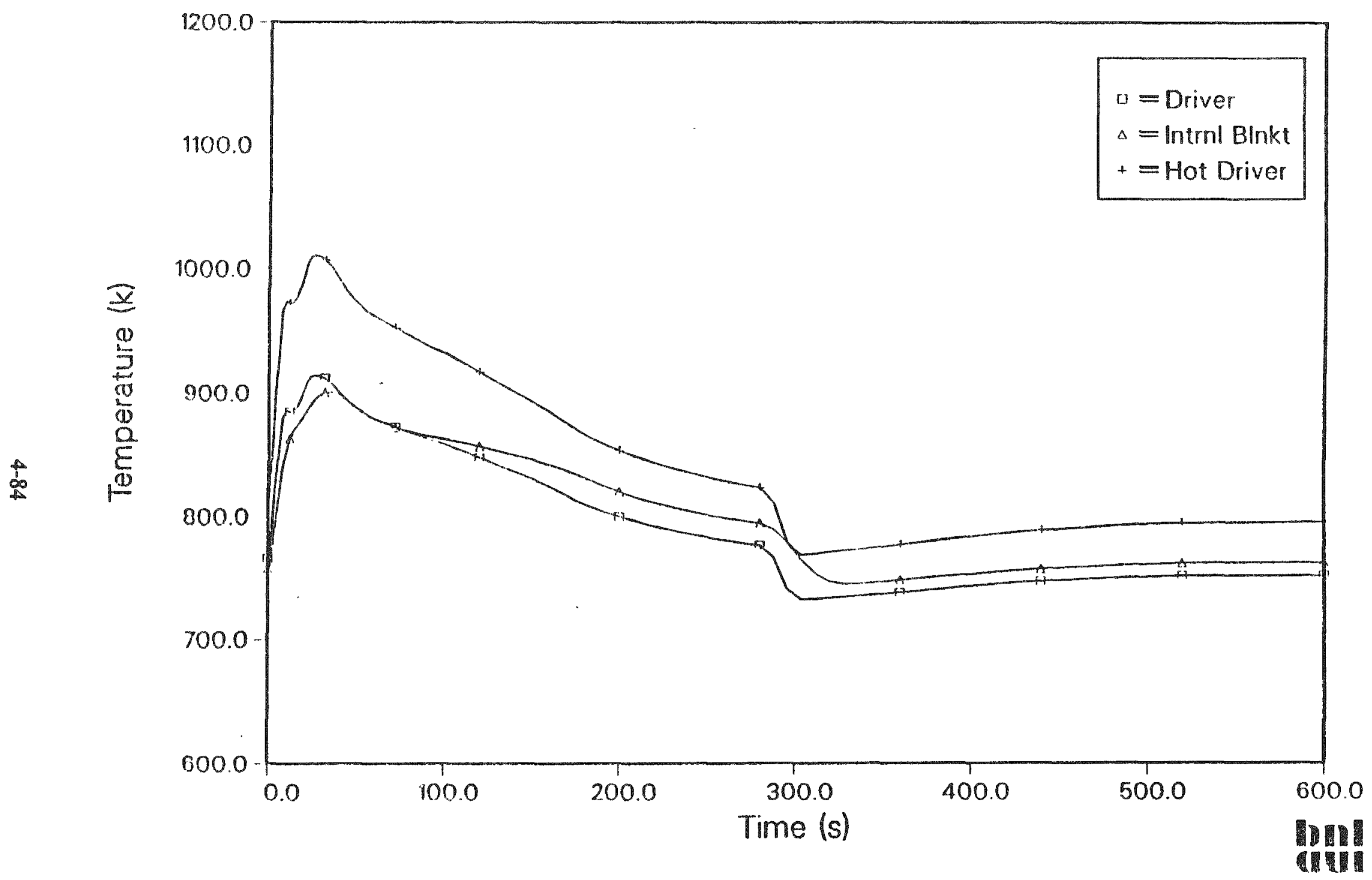

Figure 43. Predicted Channel Exit Sodium Temperatures During a 40 Cent UTOP/LOF. 


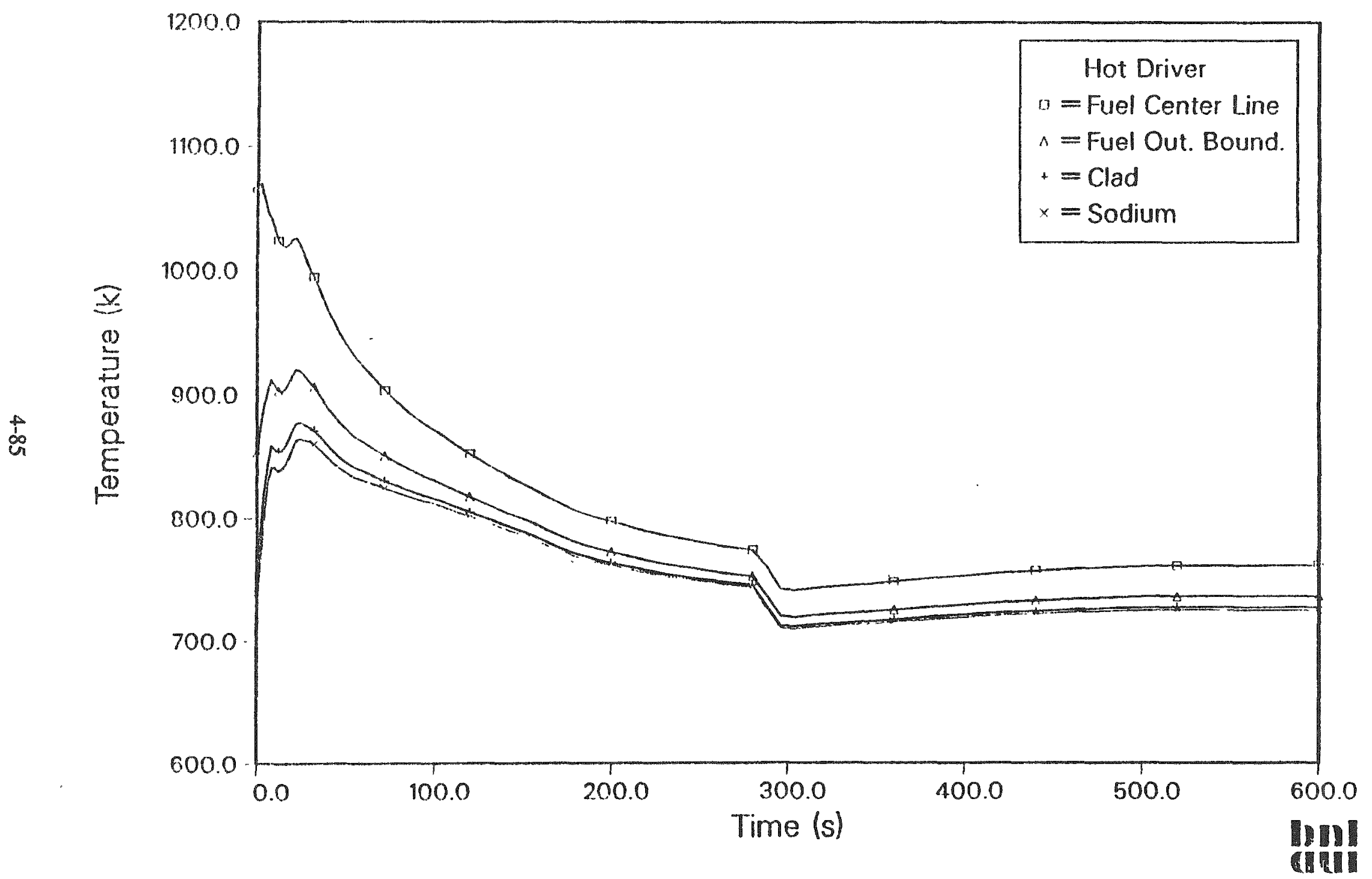

Figure 44. Predicted Fuel Temperature Distribution from SSC for the Third Node from the Top (i.e., $0.897 \mathrm{~m}-0.673 \mathrm{~m}$ ) During a 40 Cent UTOP/LOF. 


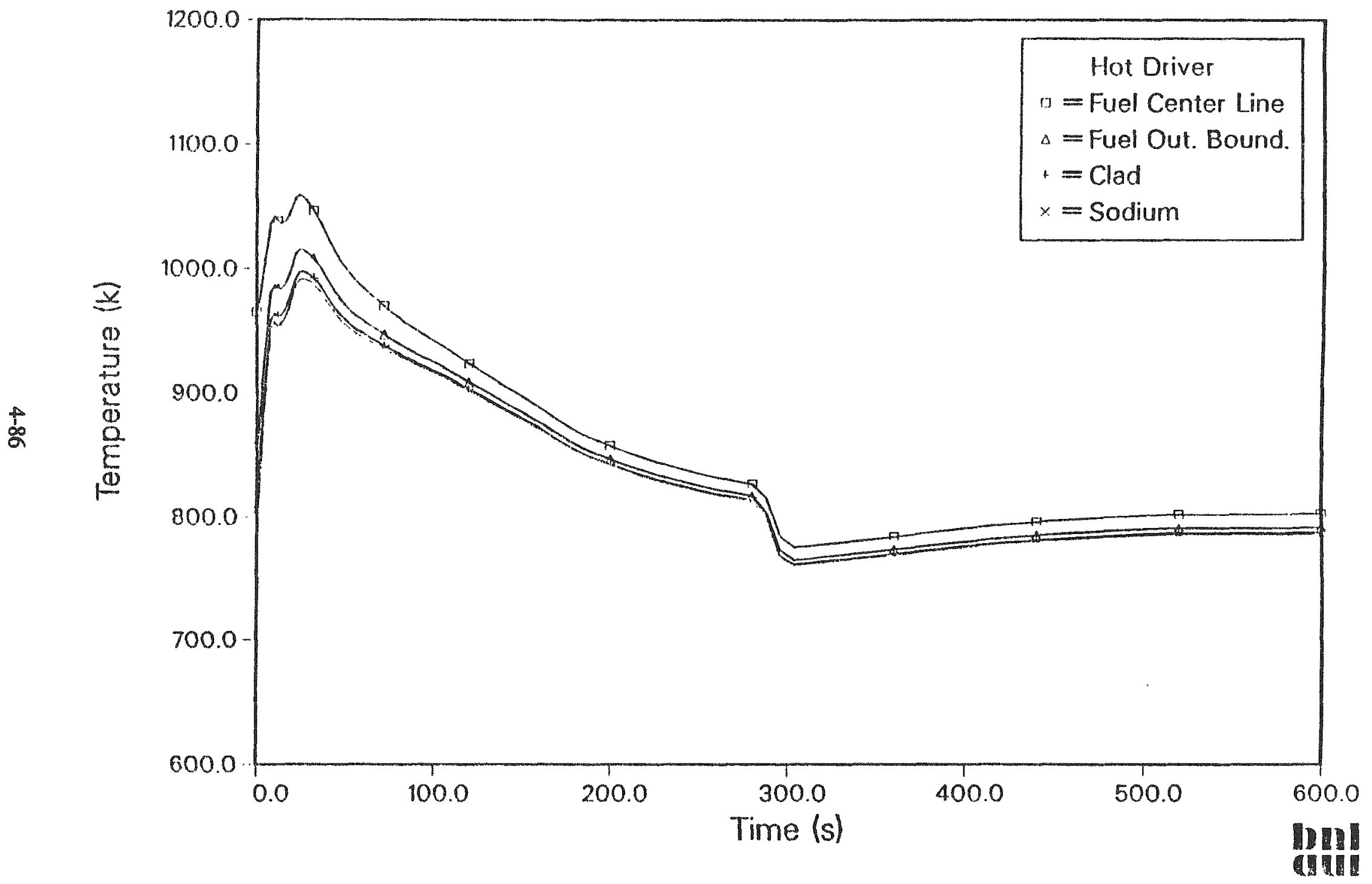

Figure 45. Predicted Fuel Temperature Distribution for the Top Node (i.e., $1.346 \mathrm{~m}-1.122 \mathrm{~m}$ ) During a 40 Cent UTOP/LOF. 


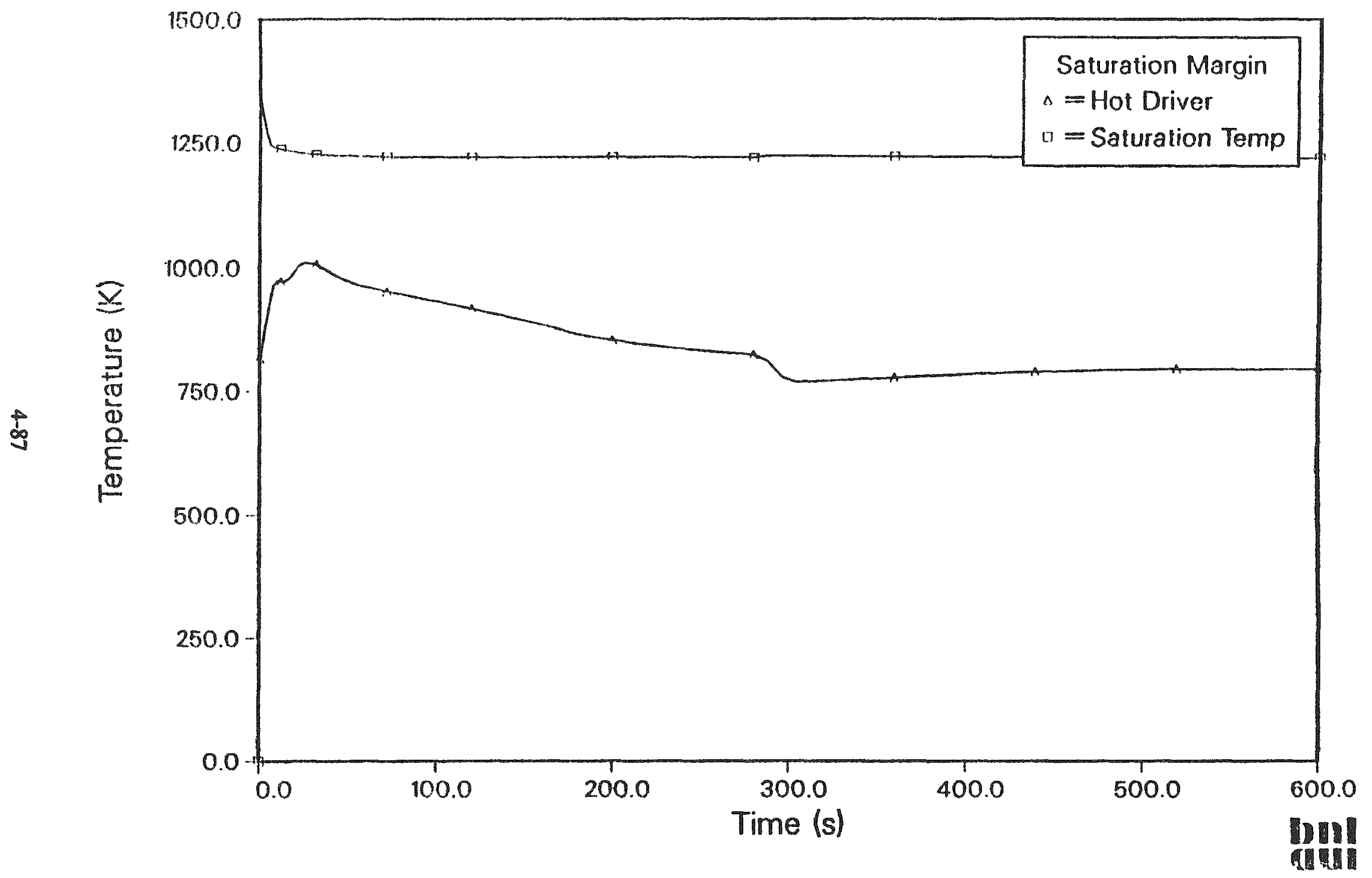

Figure 46. Predicted Sodium Saturation Margin from SSC for a 40 Cent UTOP/LOF. 


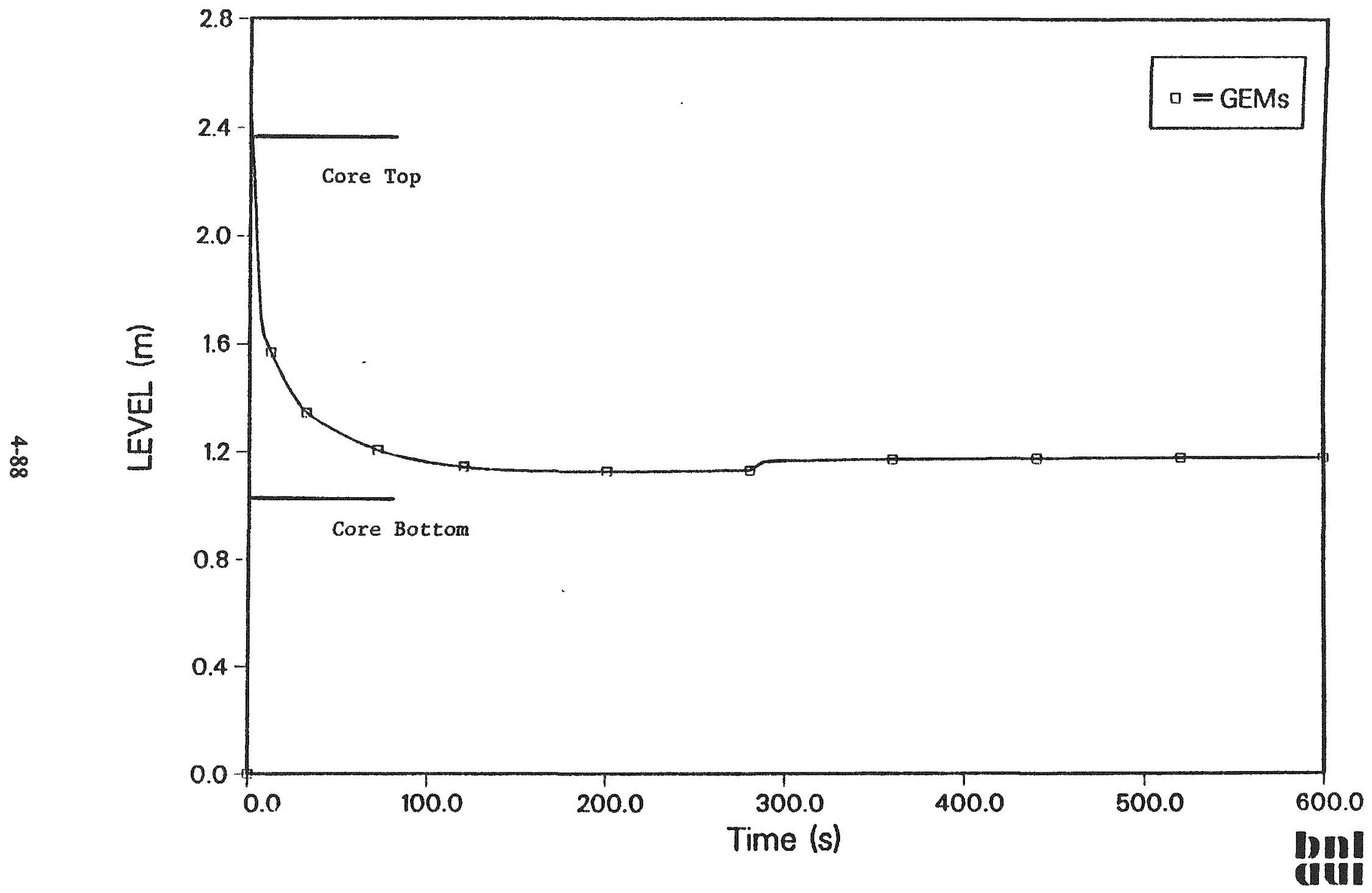

Figure 47. Predicted Sodium Level in the GEMs from SSC for a 40 Cent UTOP/LOF. 


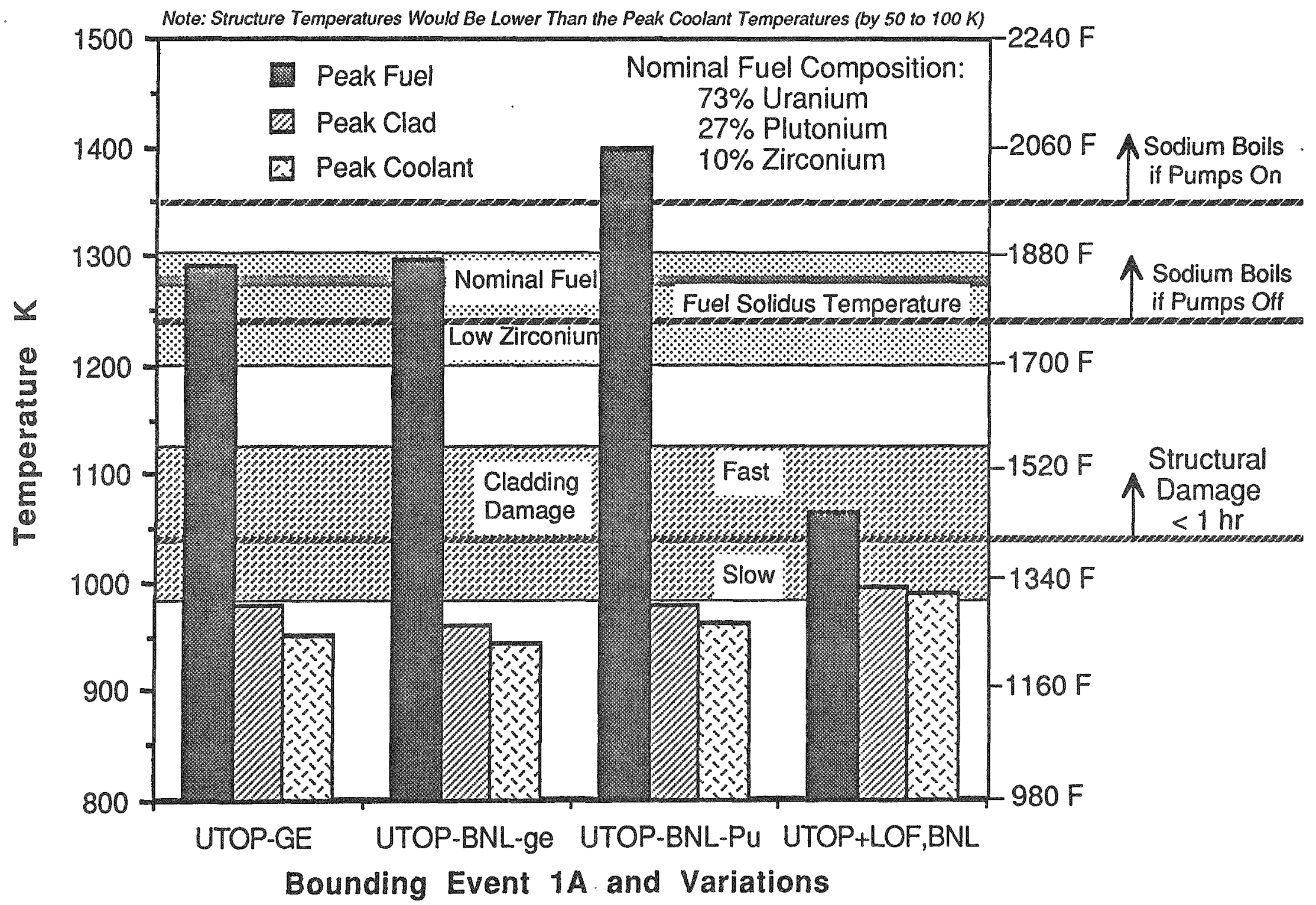

Figure 48. Peak Fuel, Clad, and Sodium Temperatures for UTOP Events: GE's 1A. BNL's 1A with and Without Pu Migration, and BNL:s UTOP/LOF 
potential cladding damage. Further, if one could be quite certain of the GEMs functioning, then the operator might intentionally trip the pumps during a UTOP event, to reduce fuel damage! - (However, this strategy could be hazardous if the GEMs fail to actuate, so it is doubtful this approach would be adopted).

\subsection{Bounding Event 2: Loss of Power}

The Bounding Event 2 defined by the NRC staff includes an assumed reactor scram, which occurs when the power is lost. The applicant stated that this event is not interesting, and proceeded to analyze an unscrammed version of Bounding Event 2. We concur with the applicant's conclusion regarding the scrammed case being uninteresting, as discussed in the next section. Their choice to analyze an unscrammed version of Bounding Event 2 is also convenient, as it offers an opportunity to reconsider some of the prior "beyond the design basis events (BDBEs)" covered in Appendix $\mathrm{E}$ of Reference 1, but not re-analyzed as part of Appendix $G$. Therefore, we have included here the analyses of a few variations on Bounding Event 2.

\subsection{With Scram}

Bounding Event 2, a loss of power with scram, does not seriously challenge the PRISM reactor system, as it is designed to passively accommodate such an event. A loss of power will cause the control rods to fall into the core, providing a fast and effective reactor shutdown. A loss of power will also result in the trip and coastdown of the system pumps. This would imply that the water inventory in the steam generators and steam drums would be available as a heat sink. Further, the ACS should provide natural draft air cooling of the exterior of the steam generators. Thus, with natural circulation in the primary and intermediate loops there should be very substantial cooling. In addition, the RVACS is an entirely passive system, and RVACS by itself is effective enough to prevent damage.

The performance of the PRISM system with scram and with RVACS providing the only cooling is discussed in Section 4.9.3. Peak sodium temperatures develop after 24 hours into the event, and are more than $40 \mathrm{~K}$ below the $\mathrm{ASME} C$ limits for the reactor vessel.

\subsection{Without Scram-Variant Cases}

The applicant chose to analyze a combined loss-of-flow and loss-of-heat-sink without scram, which is roughly equivalent to a loss of all pumping without scram, neglecting the reduced rate of heat removal through the intermediate loop based on natural circulation. We analyzed a similar event, as discussed in the next section. The variant cases covered in the two sections that follow represent a loss of primary pumping (ULOF), and a loss of all heat removal through the intermediate loop (ULOHS) which might occur if a large sodium-water reaction caused the dumping of the intermediate loop sodium.

\subsection{1 Combined ULOF/ULOHS}

This transient is initiated from full power conditions, as defined in Table 1. The transient is initiated by the EM tripping and beginning to coast down, while the IHXs stop removing heat from the primary loop. The reactor does not scram.

As in all flow coastdown transients, the likelihood of the fuel remaining undamaged is directly related to the power to flow ratio. As long as enough coolant flow is available to remove the generated heat, the fuel temperature can be maintained at acceptable levels. Figure 49 shows that the reactor power level in PRISM decreases with the flow rate. By $300 \mathrm{~s}$, the power level drops to about the decay heat 


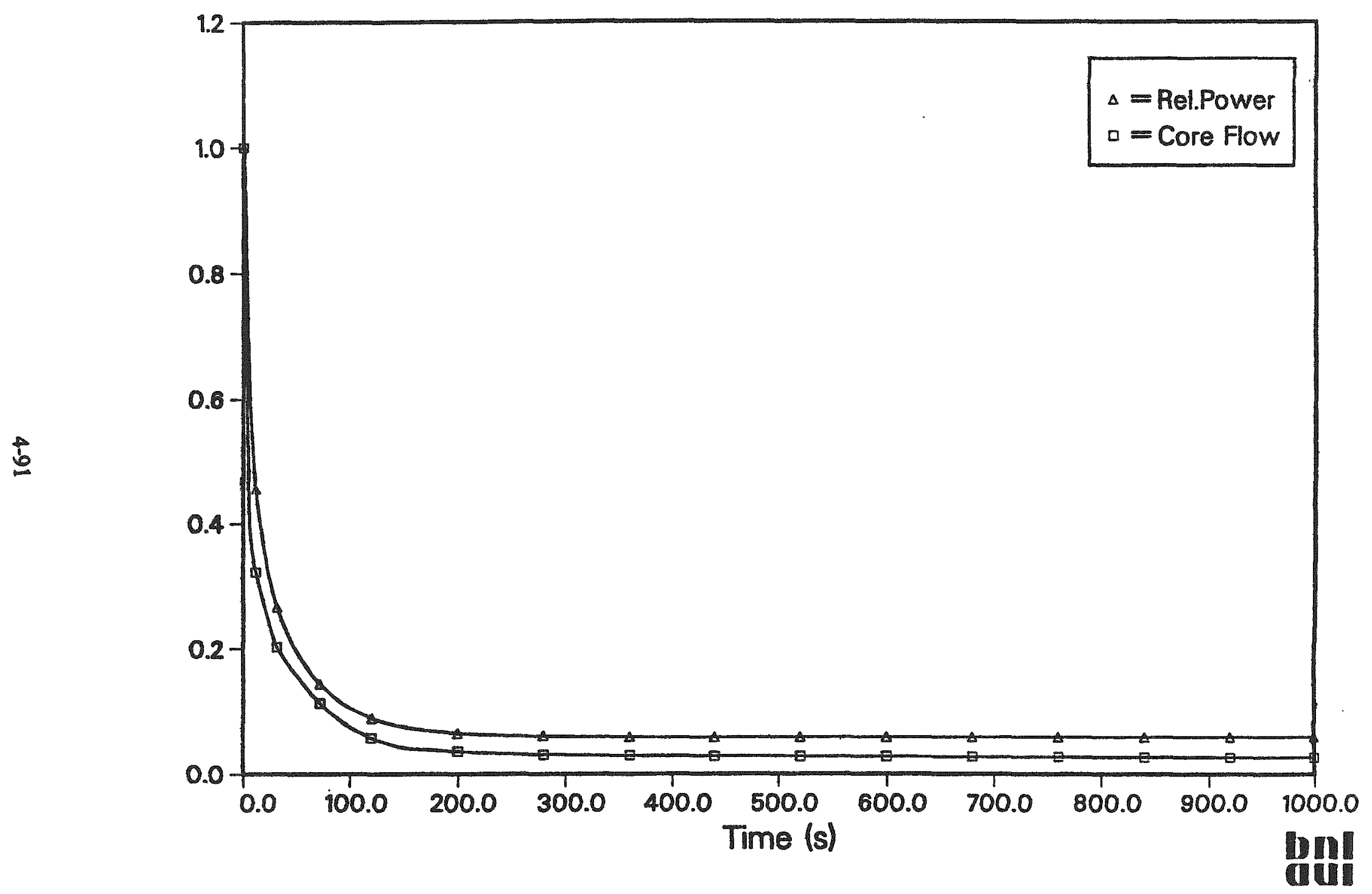

Figure 49. Predicted Relative Power and Core Flow from SSC During a ILOFFI.OHS. 
level. The neutronic feedbacks, which reduce the power, are related to the power-to-flow ratio, which determines the sodium temperature in the core. The core outlet temperature reaches $950 \mathrm{~K}$ at the end of the 1000 s, as shown in Figure 50.

In Figure 51, the total reactivity is plotted. At the end of 1000 seconds, the net reactivity is near $-\$ 1.15$. Figure 52 shows three of the components of the total reactivity. The sodium feedback reaches about +15 cents, while the axial expansion reaches about -5 cents, and the radial expansion goes to -16 cents. The components of the radial expansion are the above core load pads (ACLP) and core grid plate, which are plotted in Figure 51. The large thermal mass of the system delays the grid plate heat-up. The dominant feedback during this event is the negative feedback from the gas expansion modules (GEMs), as shown in Figure 53. The Doppler feedback, also plotted in Figure 53, shows a positive response because the GEMs reduce the power so quickly that the fuel actually cools down and does not heat up enough to give a negative feedback until after $400 \mathrm{~s}$ into the transient.

The 3 GEMs have a total reactivity worth of -69 cents. During the ULOF, the gas region drops into the core region as the pressure in the inlet plenum decreases. The fast insertion of negative reactivity reduces the power, keeping the power-to-flow-ratio favorable, so the heat can be removed without fuel damage. The drop in the GEM sodium level with core inlet pressure (or pump coast down) can be seen in Figure 54 and 55, with the corresponding reactivity insertion included in Figure 53 . It can be seen that the GEMs quickly add $-66 \mathrm{c}$ by $100 \mathrm{~s}$, reducing the power nearly as quickly.

The fuel temperatures drop very quickly at the core center. During the transient, the peak fuel temperatures shift to the core exit, where the peak sodium temperatures cause the highest fuel temperature. In Figure 56, the temperatures at the exit of the hot channel are shown. The fuel reaches a temperature of $990 \mathrm{~K}$ by $1000 \mathrm{~s}$, and is in a range where eutectic penetration would begin. Finally, the margin to boiling is shown in Figure 57, which indicates the closest margin to boiling is near the end of the simulation period, and is $215 \mathrm{~K}$ from boiling. This margin may decrease, depending on the duration of the heat up.

\subsection{2 ULOF Only}

The ULOF is initiated by a trip and coastdown of the EM pumps from full power. The initial conditions corresponding to full power are as shown in Table 1. Two cases were examined, namely with and without GEMs.

\section{With GEMs Case}

The power immediately begins to drop, as shown in Figure 58, and reaches decay heat by 500 $s$, since there is enough negative reactivity at these temperatures to keep the core subcritical. The core average sodium outlet temperature, shown in Figure 59 , reaches a peak of $830 \mathrm{~K}$. The peak temperatures from the hot driver are plotted in Figures 60 (center) and 61 (top). In Figure 62, the net reactivity is plotted. The reactivity contribution from GEMs is shown in Figure 64. The GEMs insert about -58c by $200 \mathrm{~s}$, but do not reach their full worth until $600 \mathrm{~s}$. This effect is caused by the increasing temperature and pressure of the cover gas, during the beginning part of the event, and higher density sodium coming in from the (still functioning) IHX. Note that in Figure 62, the radial expansion components, i.e., the above core load pads (ACLP) and core bottom grid plate, turn positive since the GEMs push the power and temperatures down. As is also shown in Figure 64, the Doppler and control rod drive line (caused from vessel expansion) feedbacks turn slightly positive. Figure 63 shows that the axial and radial expansion feedbacks are positive, while the usually positive feedback from sodium density goes negative a few cents, due to the reduction in average sodium temperature, as referenced from 


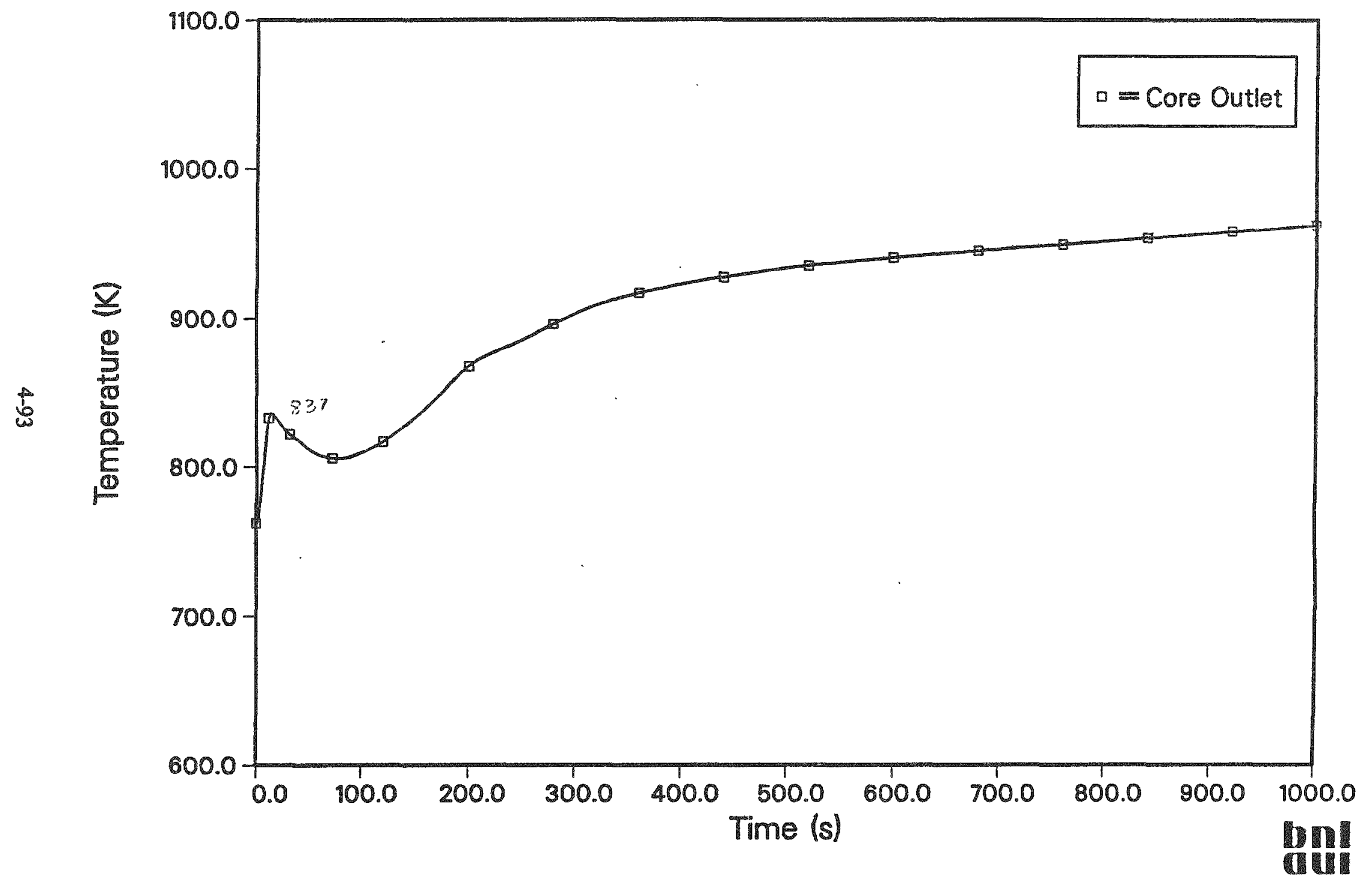

Figure 50. Average Core Outlet Temperature Prediction from SSC During a ULOF/LOHS. 


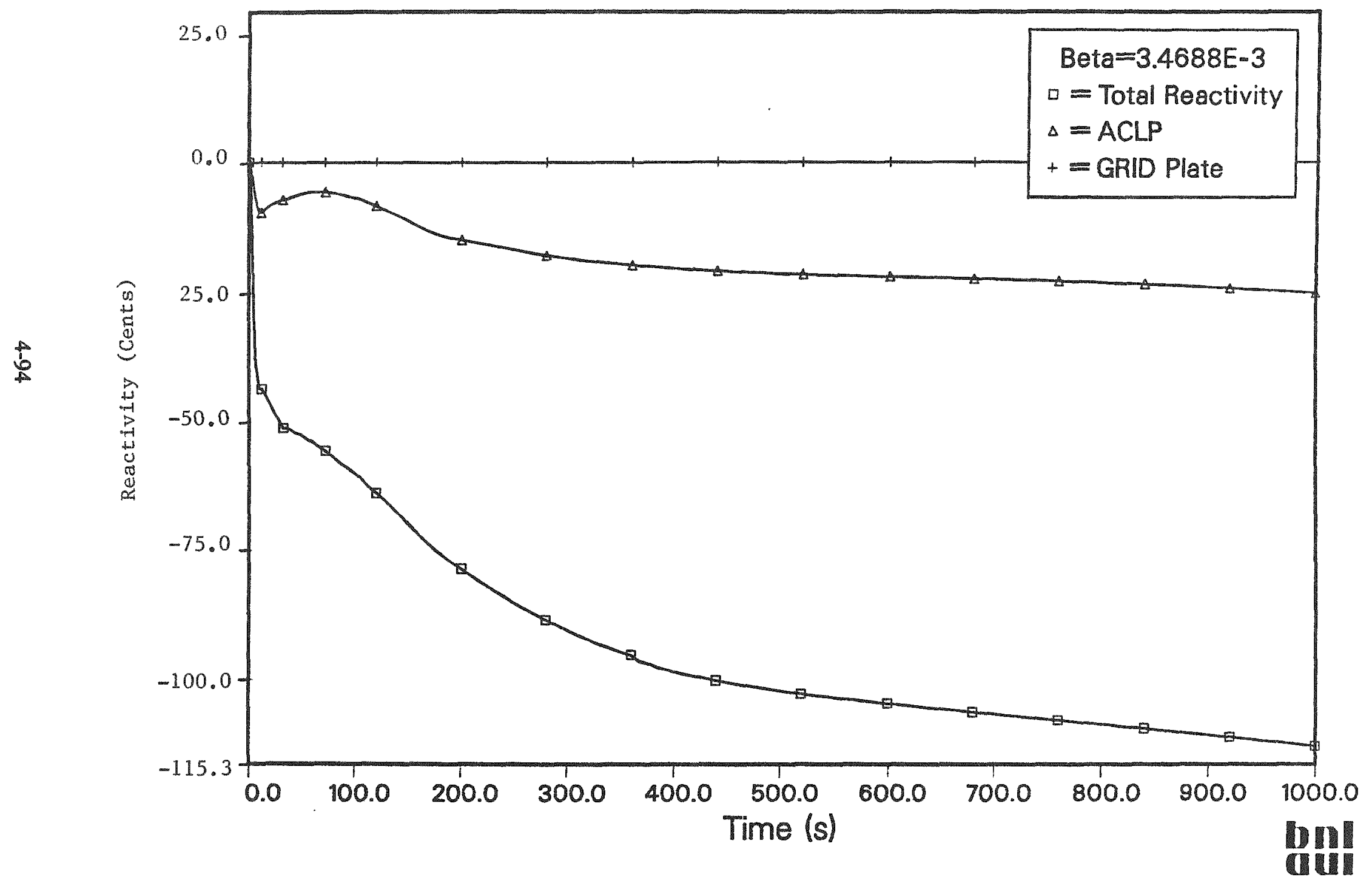

Figure 51. Predicted Total, Above Core Load Pad (ACLP) and Core Support Grid Plate Reactivity Feedback from SSC for a 40 Cent UTOP/LOHS. 


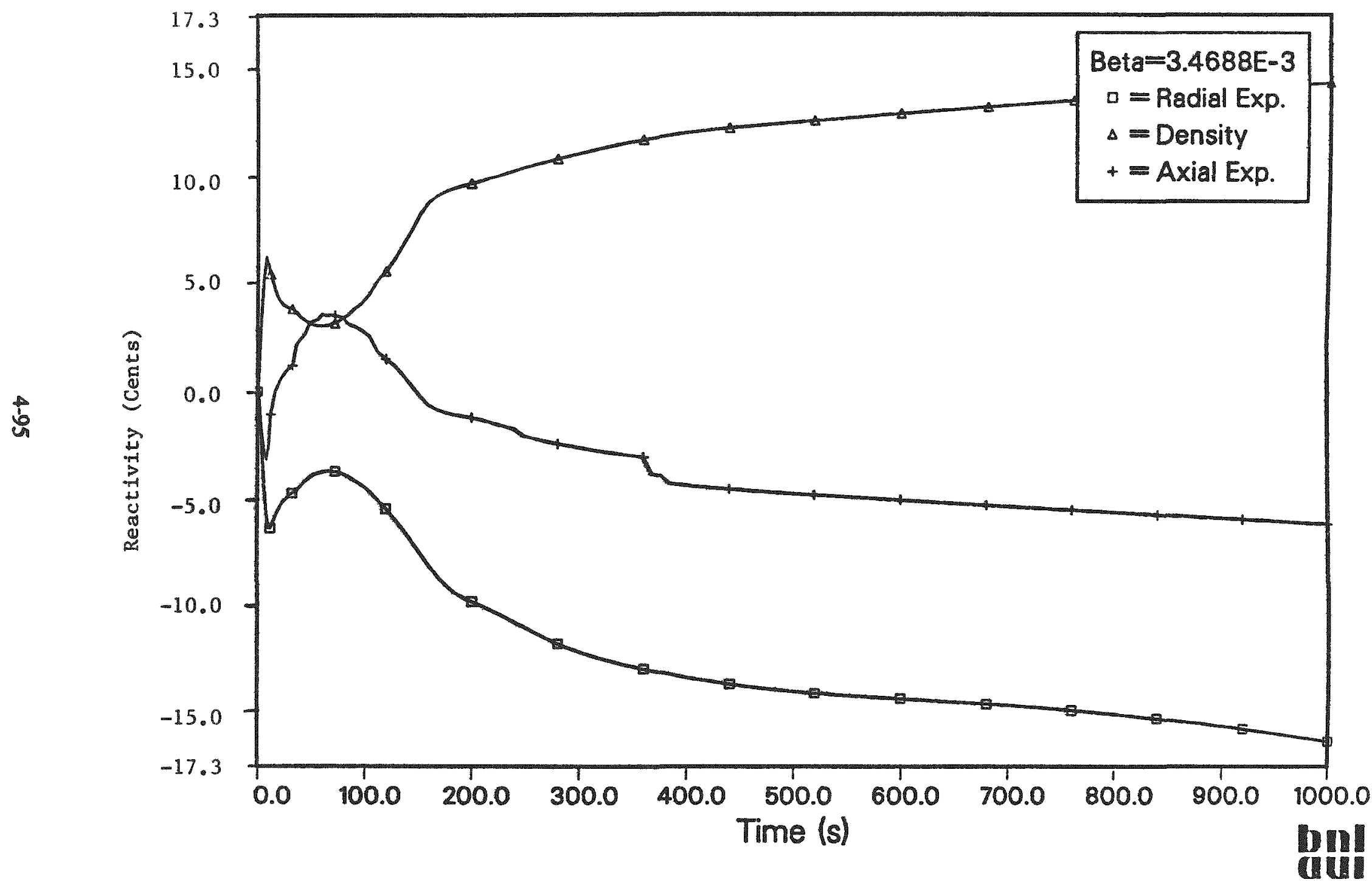

Figure 52. Predicted Radial Expansion, Sodium Density, and Axial Expansion Reactivity Feedback from SSC During a ULOF/LOHS. 


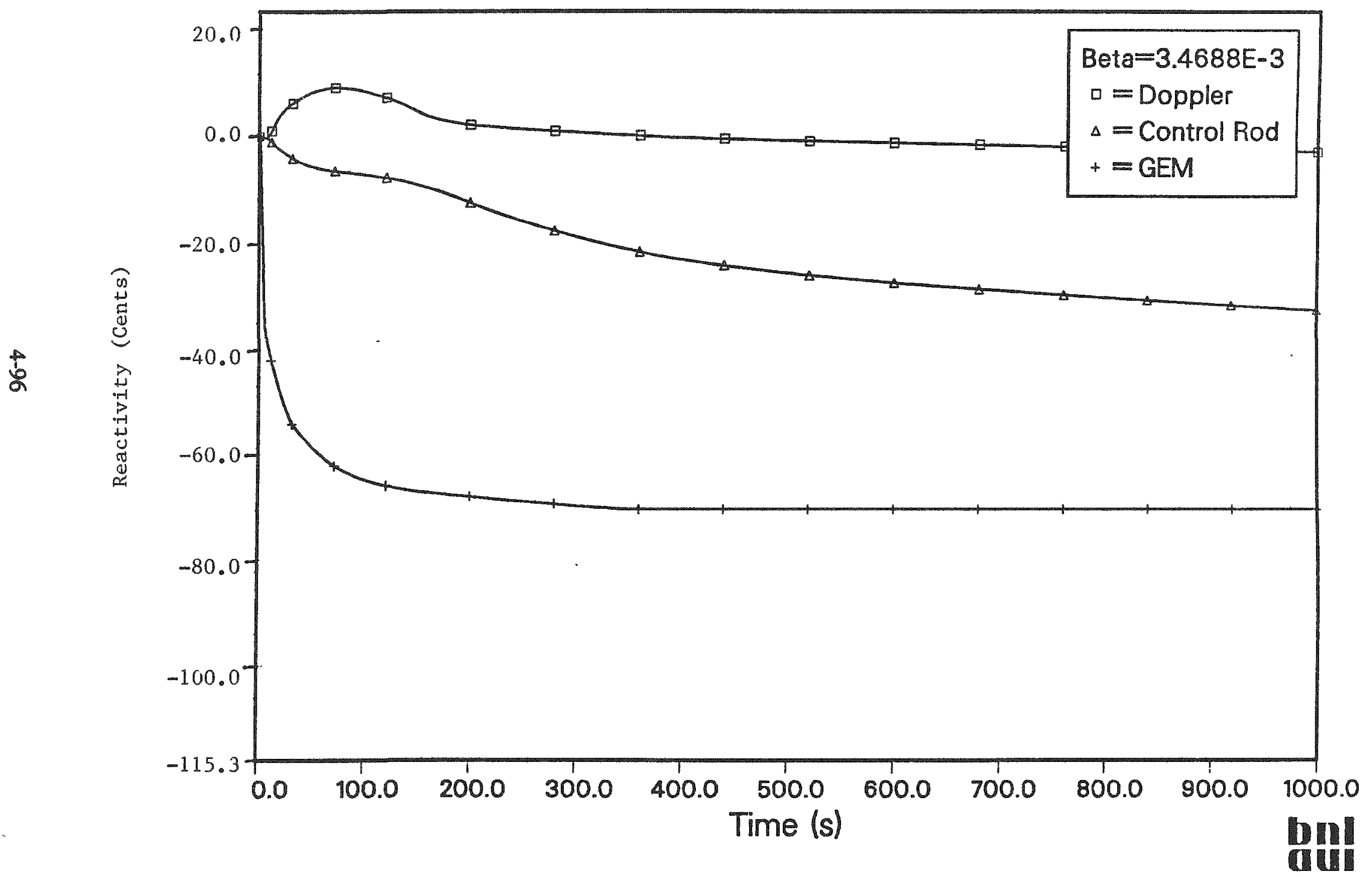

Figure 53. Predicted Doppler, Control Rod Expansion, and Gas Expansion Module (GEM) Reactivity Feedback from SSC during a 40 Cent ULOF/LOHS. 


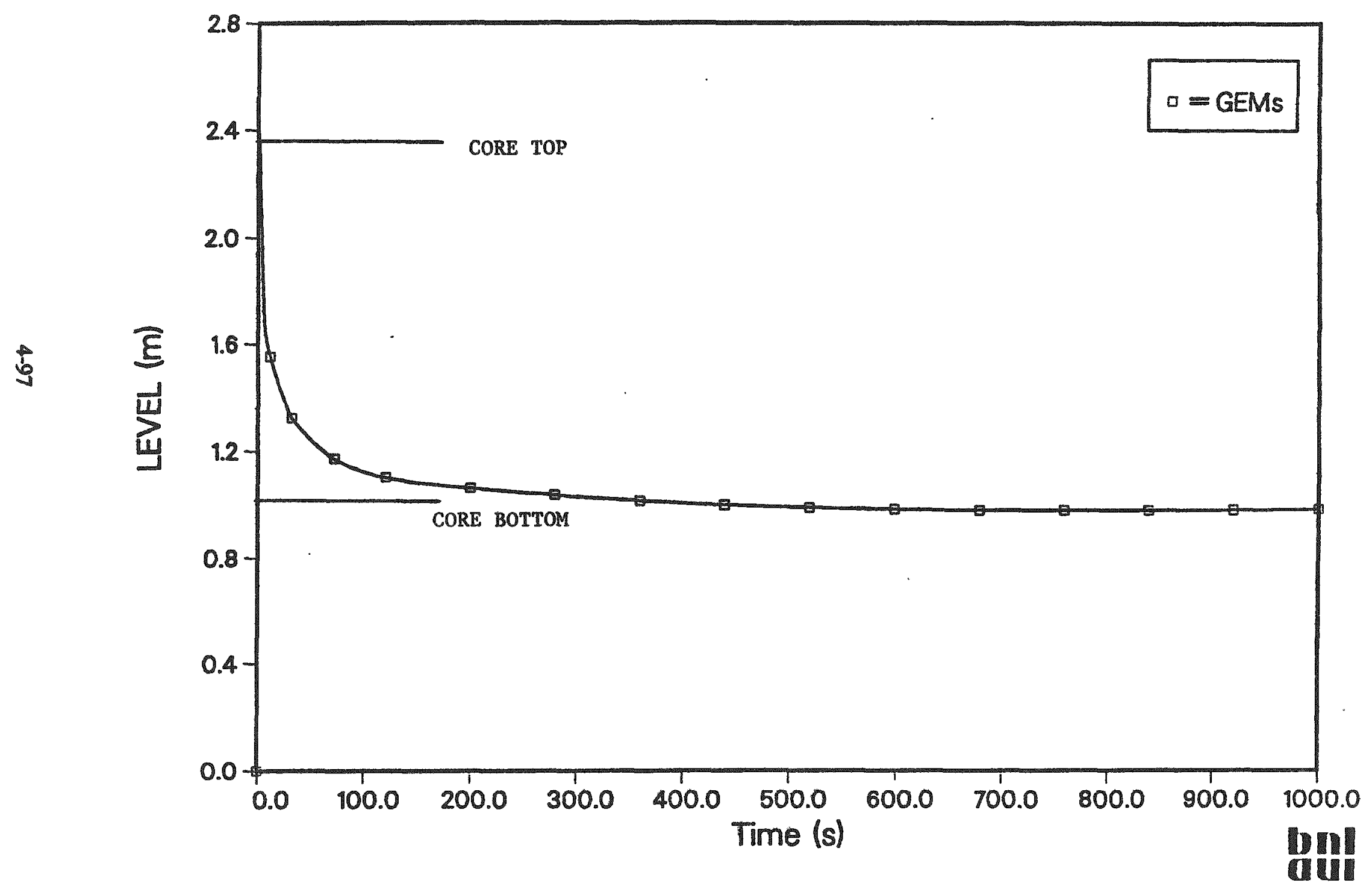

Figure 54. Predicted Sodium Level in the GEMs from SSC During a ULOF/LOHS. 


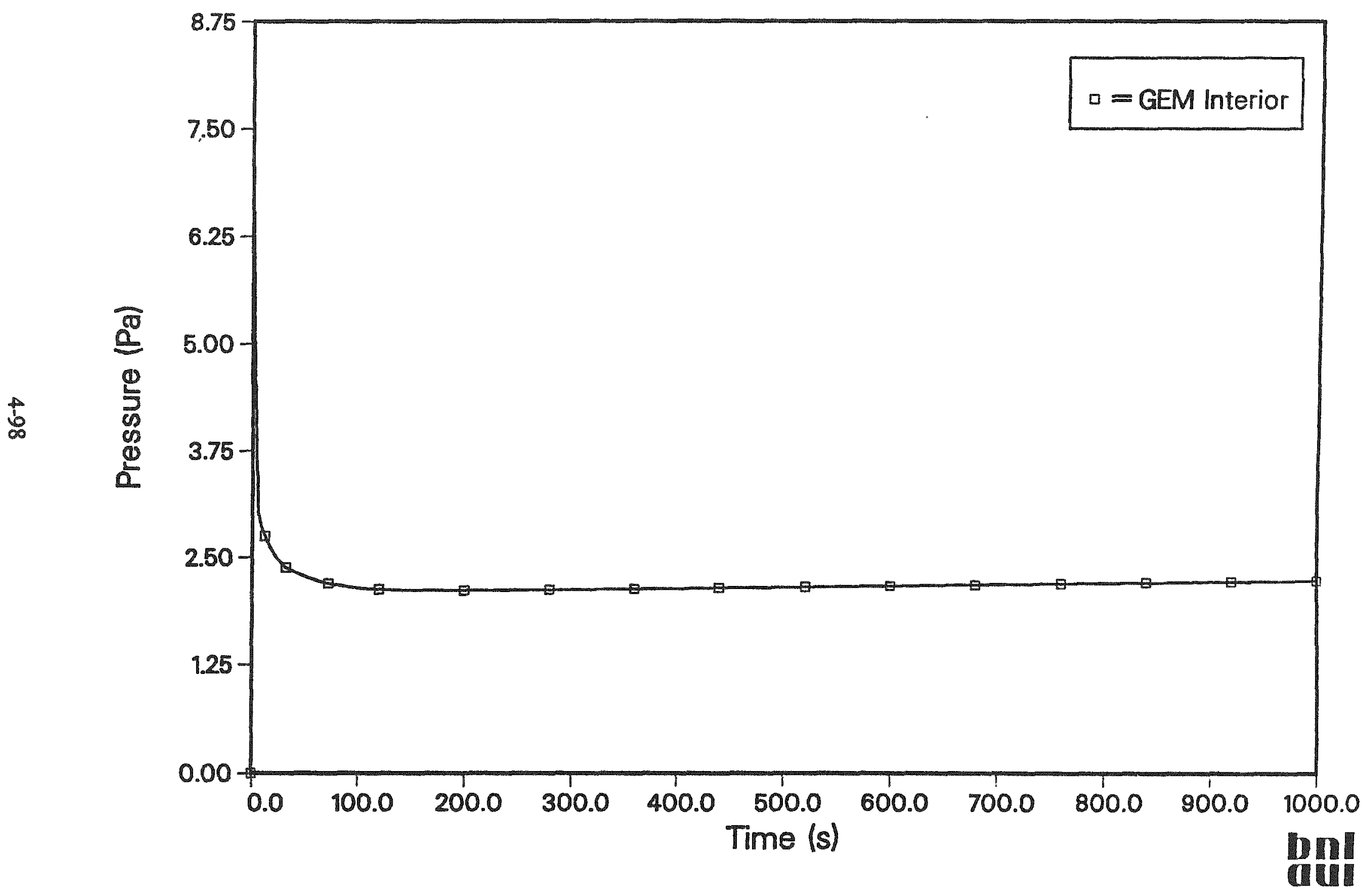

Figure 55. Predicted GEM Gas Pressure from SSC During a ULOF/LOHS. 


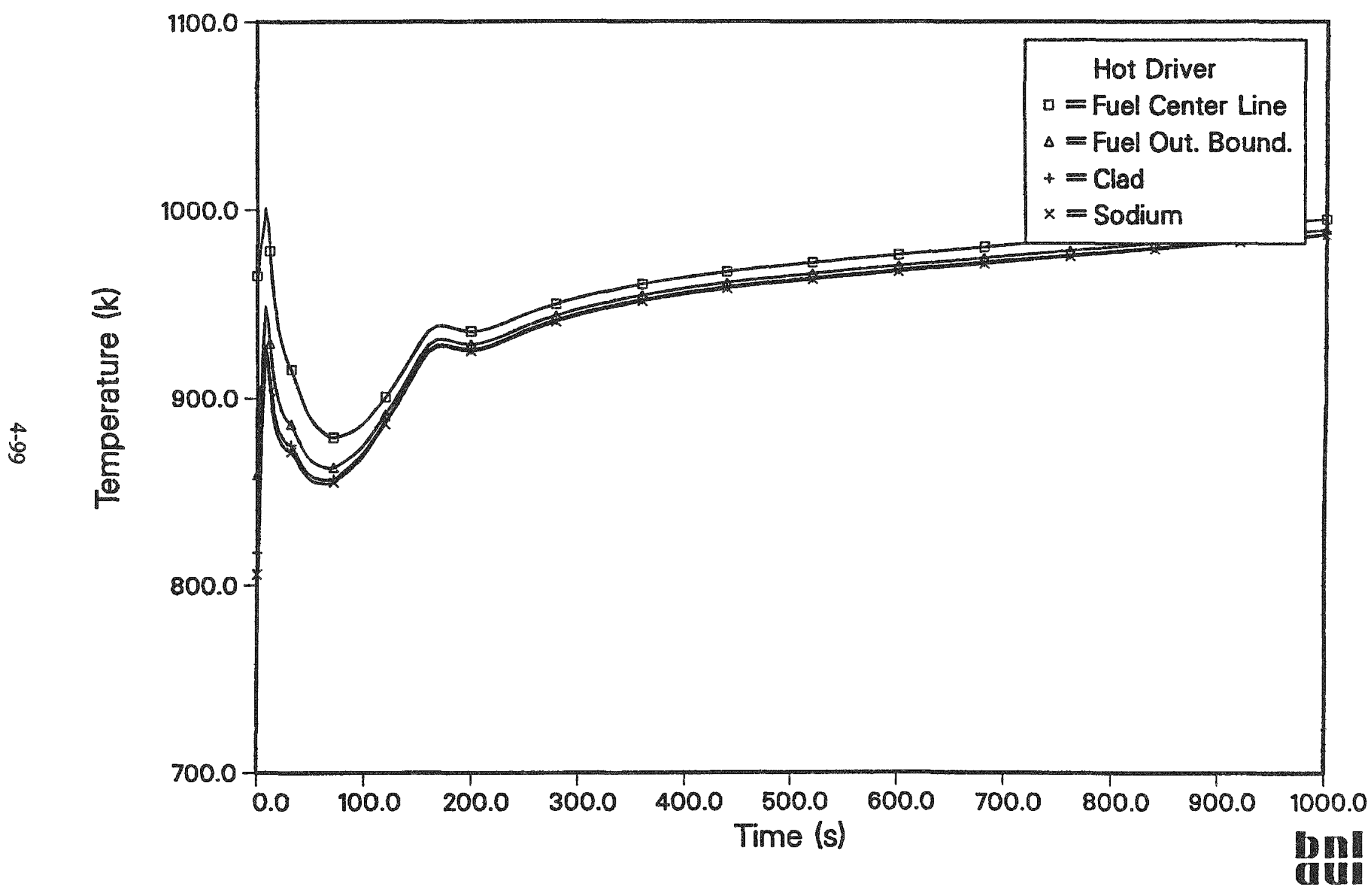

Figure 56. Predicted Fuel Temperature Distribution from SSC for the Top Node (i.e., $1.346 \mathrm{~m}$ $1.122 \mathrm{~m}$ ) During a ULOF/LOHS. 


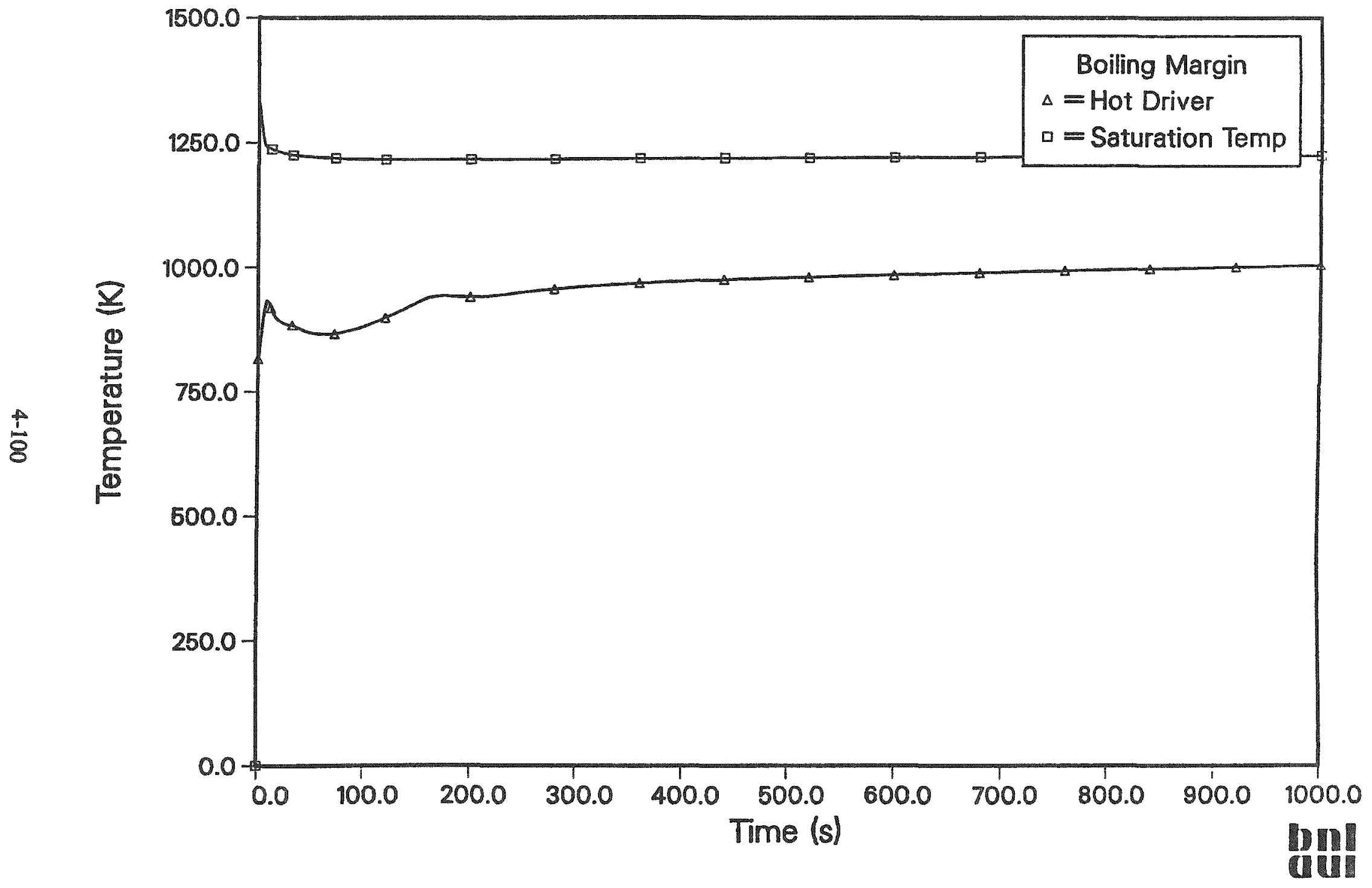

Figure 57. Predicted Sodium Saturation Margin from SSC During a ULOF/LOHS. 


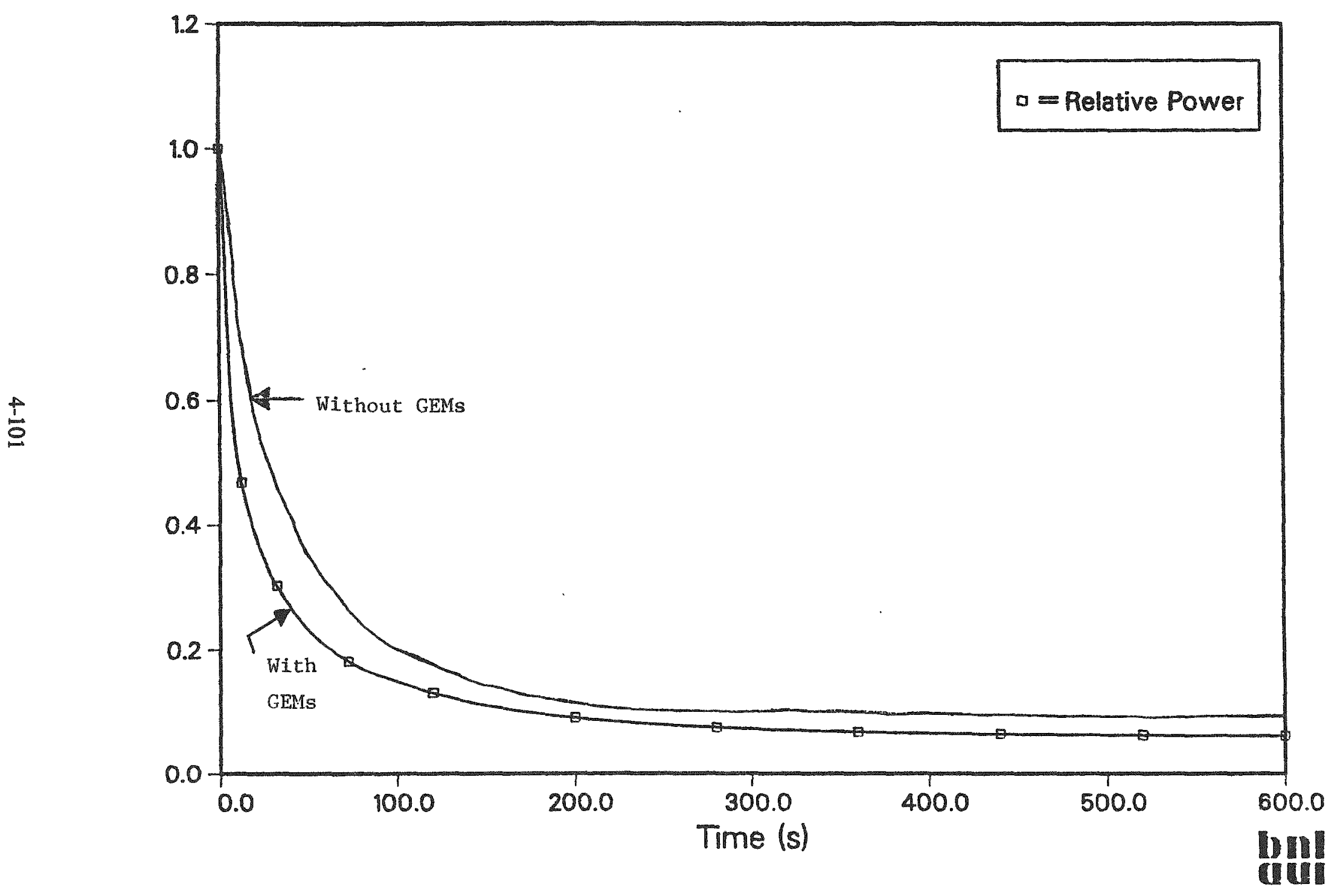

Figure 58. Predicted Relative Power from SSC for a ULOF with and without GEMs. 


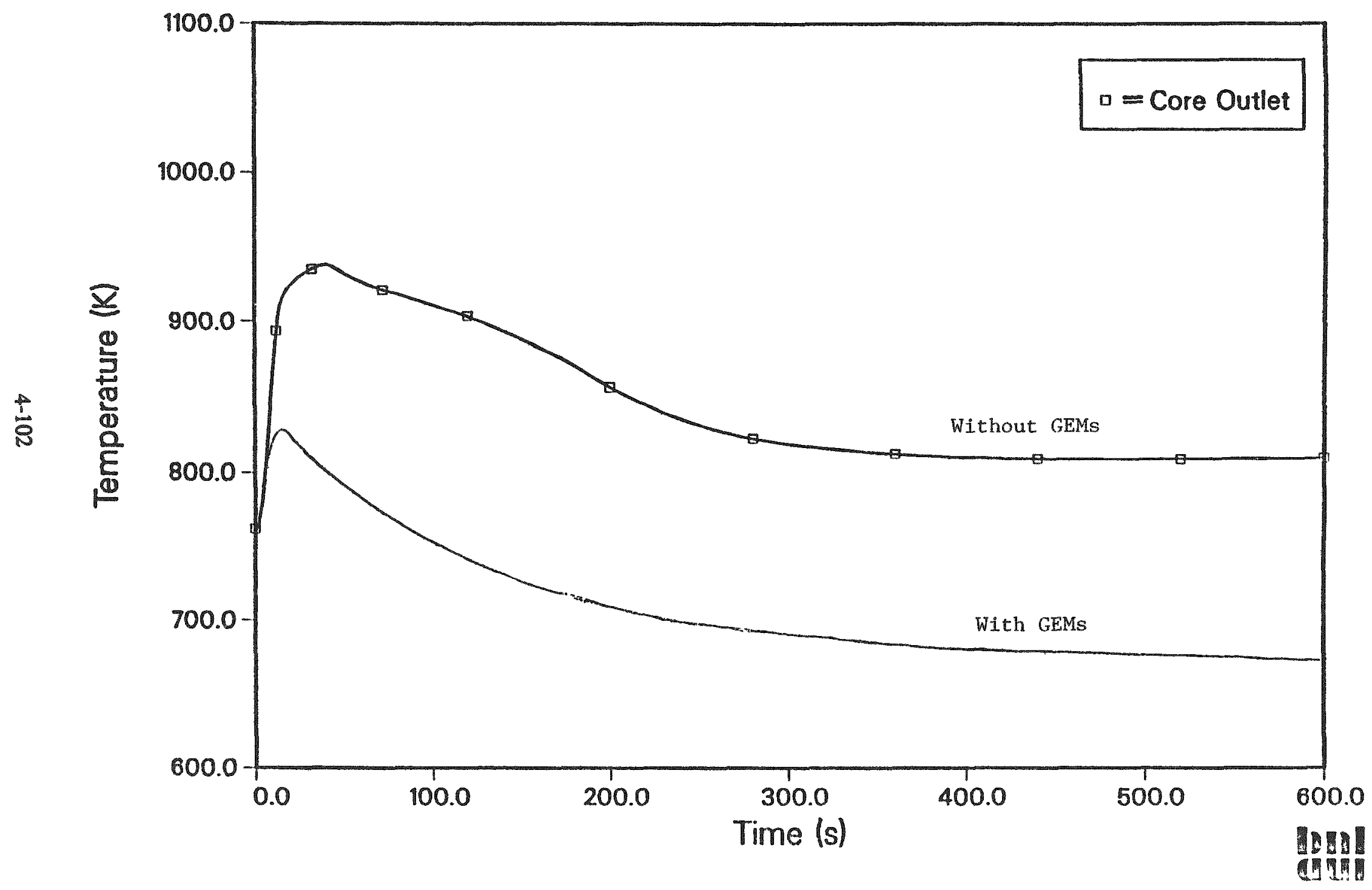

Figure 59. Average Core Outlet Temperature from SSC for a ULOF With and Without GEMs. 


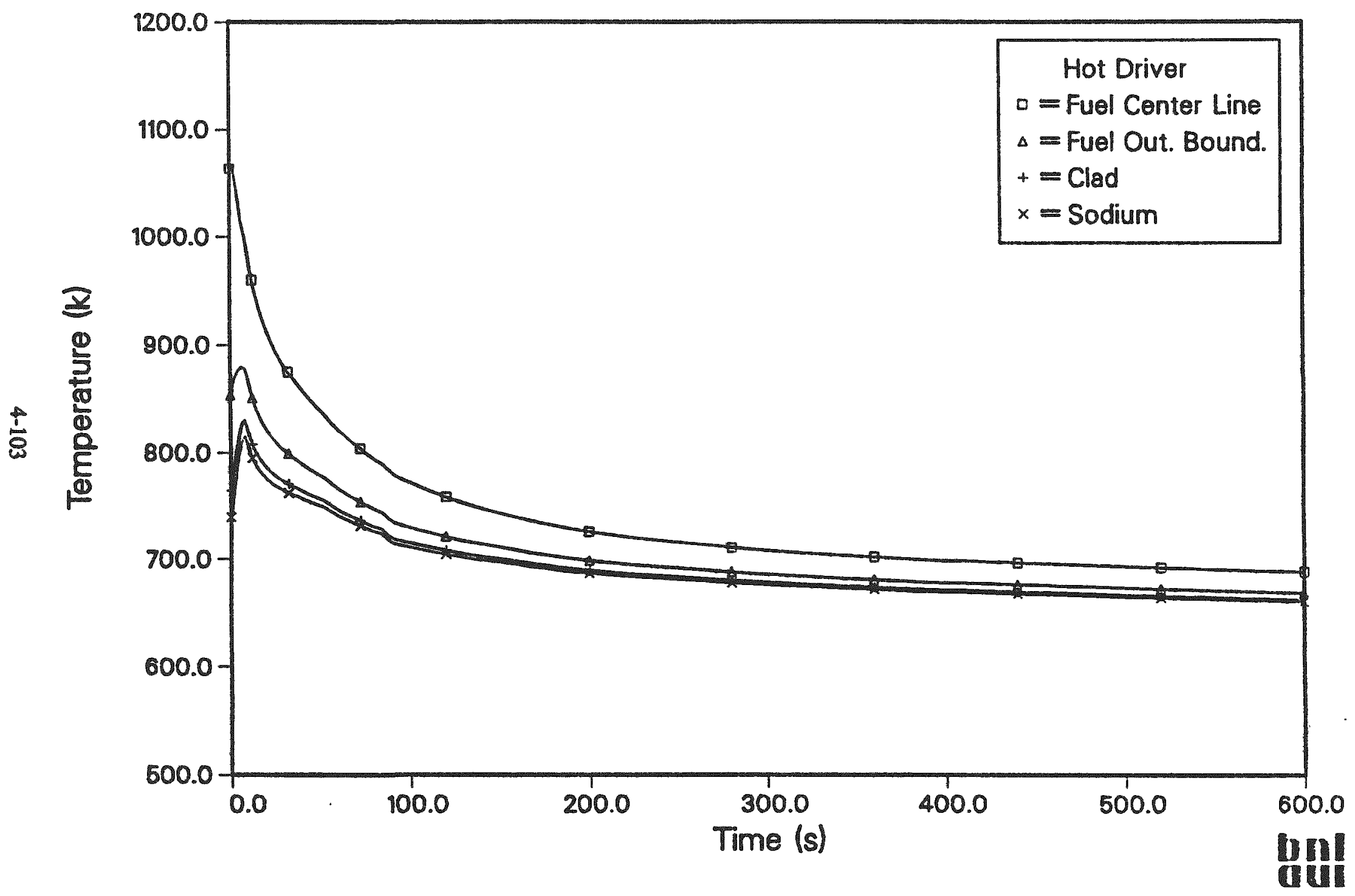

Figure 60. Predicted Fuel Temperature Distribution from SSC for the Third Node from the Top (i.e., $0.897 \mathrm{~m}-0.673 \mathrm{~m}$ ) for a ULOF with GEMs. 


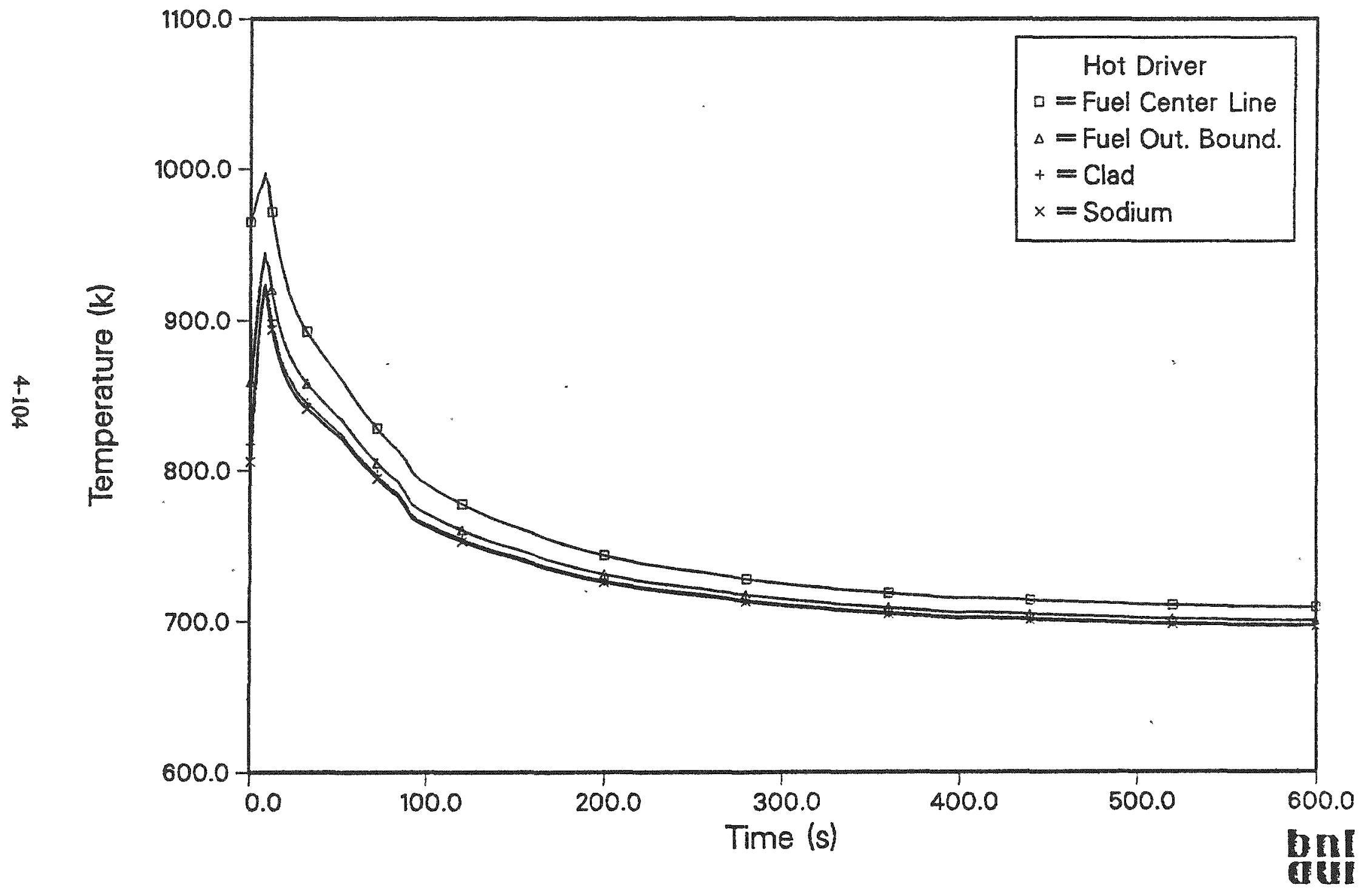

Figure 61. Predicted Fuel Temperature Distribution from SSC for the Top Node (i.e., $1.346 \mathrm{~m}-1.122 \mathrm{~m}$ ) for a ULOF with GEMs. 


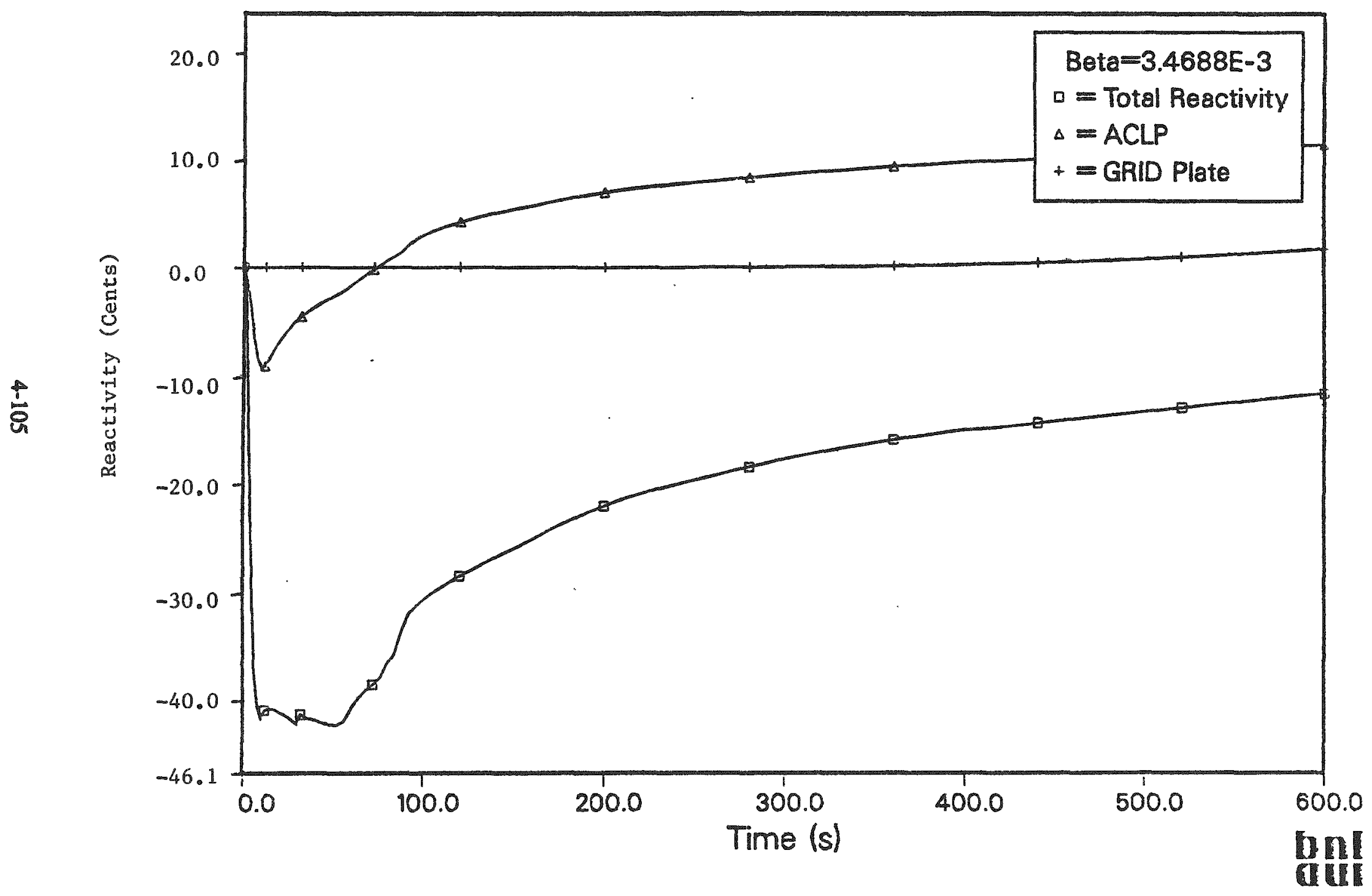

Figure 62. Predicted Total, ACLP, and GRID Plate Reactivity Feedback from SSC for a ULOF with GEMs. 


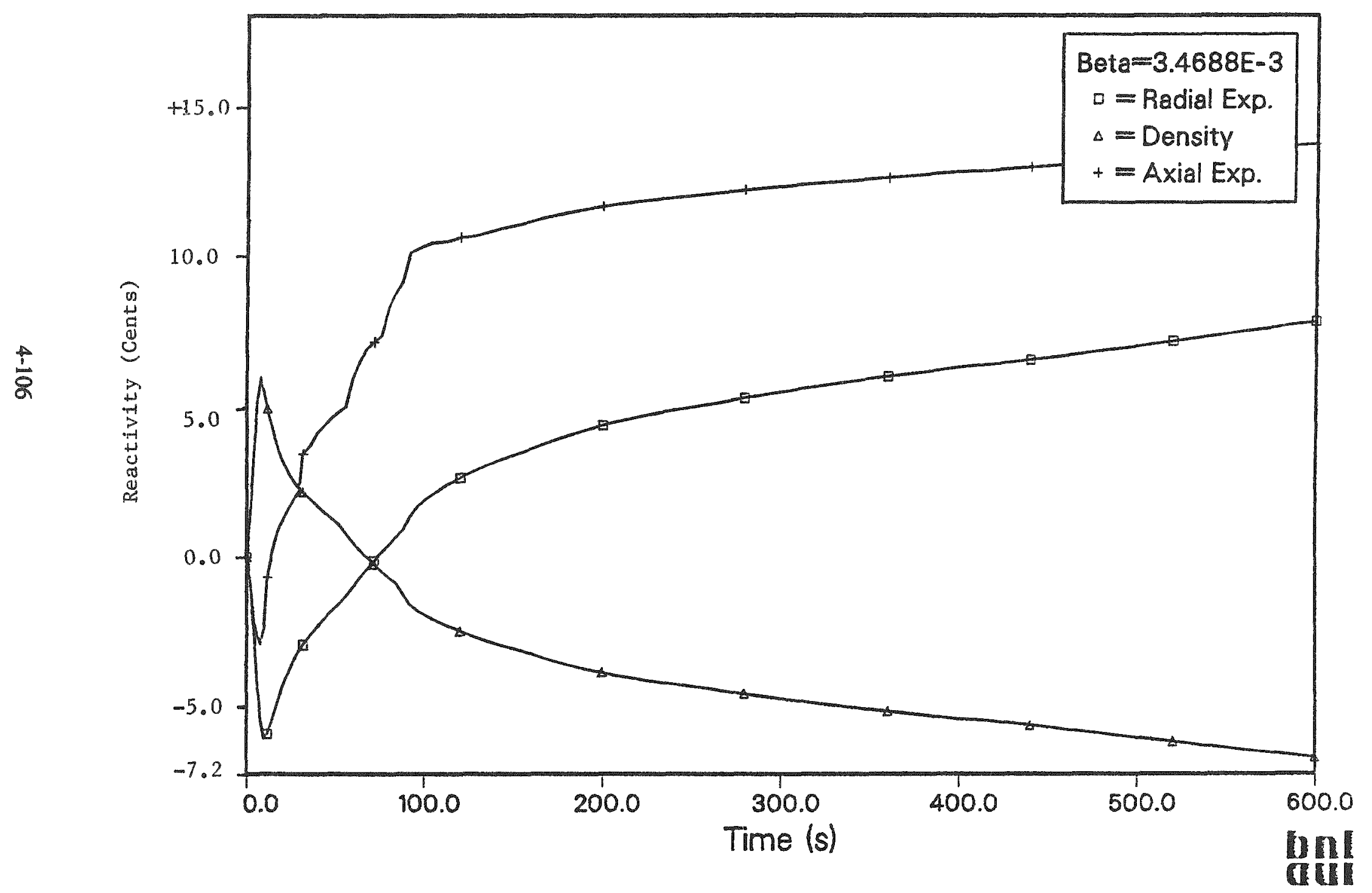

Figure 63. Predicted Radial Expansion, Sodium Density, and Axial Expansion Reactivity Feedback from SSC for a ULOF with GEMs. 
'SWAD YIIM HOTA ROJ

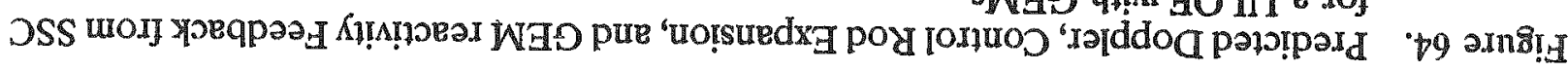

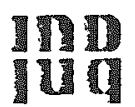

(s) әแ!

0.000

0.009

0.007

$0.00 \varepsilon$

0.002

0.001

0.0

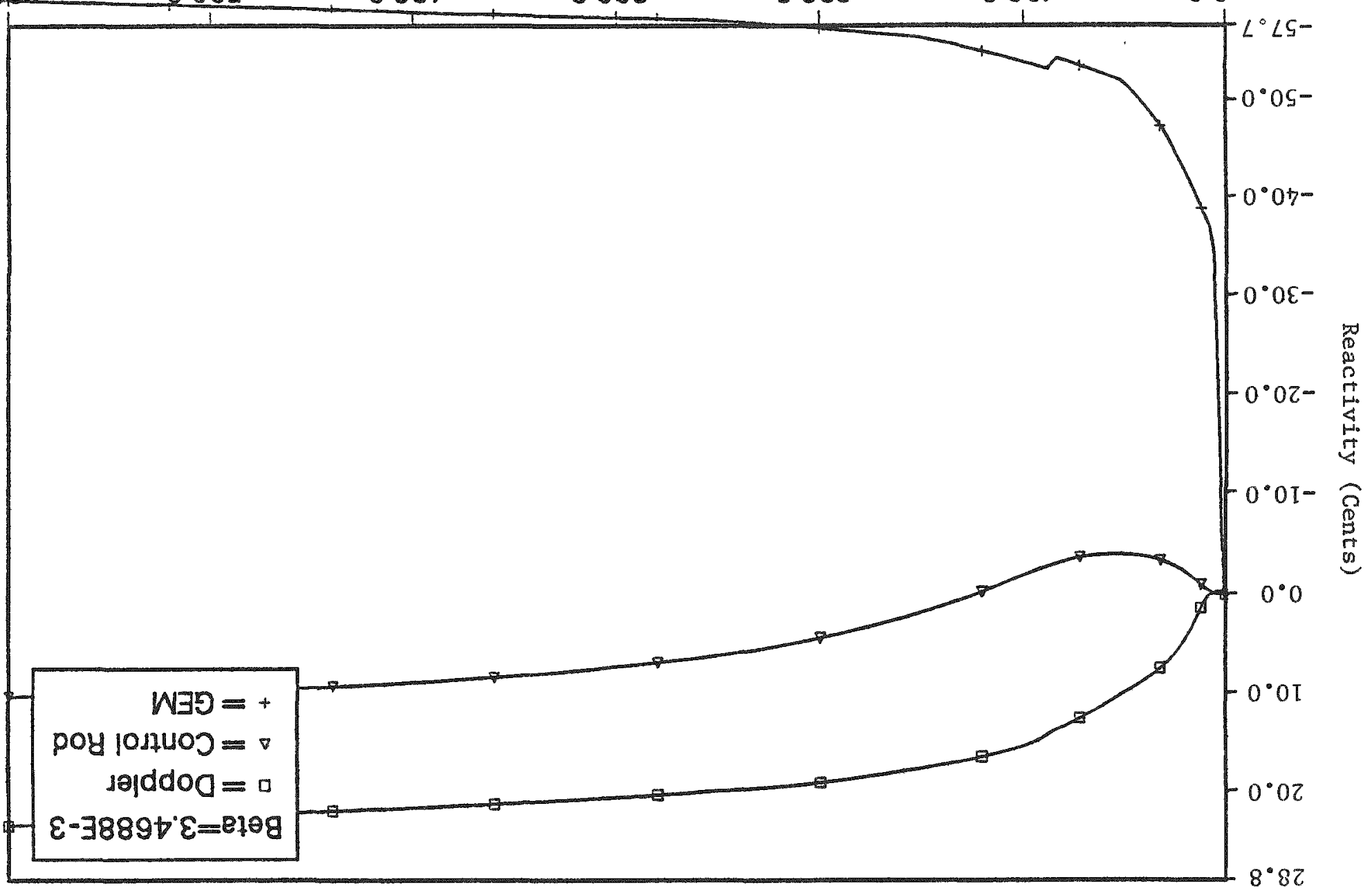


nominal operating conditions. While the feedbacks respond differently to achieve the power reduction, as compared to the previous transients, enough negative reactivity is inserted to drive the core subcritical, and the power transitions down to the decay heat level by $500 \mathrm{~s}$. The last potential point of concern is shown in Figure 65 where the peak channel outlet sodium temperature is plotted against the sodium saturation temperature. The margin to boiling with the GEMs is approximately $300 \mathrm{~K}$.

The ULOF without GEMs would be a power reduction from the temperature dependent reactivity. The predicted power is plotted along with the power predicted for the case with GEMs, shown in Figure 58. It is noted that without the GEMs the power is slightly higher and decreases somewhat slower, since temperature increases must activate the reactivity feedbacks. The average core outlet sodium temperature increases to $935 \mathrm{~K}$ ( $830 \mathrm{~K}$ with GEMs) and levels out at about $850 \mathrm{~K}$. These higher temperatures are needed to match the heat loss and the power production, which is higher than decay heat levels. As shown in Figure 66, the net reactivity peaks near -27 cents. Note that the ACLP contribution is negative, since the core without GEMs must heat up significuntly. Figures 67 and 68 include other calculated neutronic feedbacks. The sodium heats up as the sodium flow rate reduces, raising system temperatures. The Doppler, axial expansion, radial expansion, and control rod drive line reactivity feedbacks initially turn negative, with only the sodium feedback positive. The net is a negative feedback initially, and the power level is later re-established at about $10 \%$ rated power. The increases in temperature also changes temperatures within the fuel. The increase in system temperatures causes an increase in the peak centerline temperamre before the power begins to fall, as shown in Figure 69 and 70. However, the peak fuel temperatures reach only $1150 \mathrm{~K}$, for the case without the GEMs. This is far below the solidus temperature, (i.e., $1249 \mathrm{~K}$ ), even factoring in the three annular rings that develop within the fuel pellet from $\mathrm{Zr}$ migration. Figure 65 shows that without the GEMS, the peak sodium temperature is only 160 $\mathrm{K}$ from boiling.

\section{Conclusions for the ULOF Cases}

The SSC predictions show that PRISM would be able to withstand the ULOF, both with and without the aid of the GEMs. The GEMs can dominate the neutronic feedbacks, and can bring the power down to the decay heat level within $500 \mathrm{~s}$, with a margin to sodium boiling of about $300 \mathrm{~K}$. The fuel temperatures decrease, and fuel damage is not a significant risk for this event. The case without the GEMs shows the usual heat up of the structures, which activates the reactivity feedbacks, thus reducing the power. This causes the power to stabilize around $10 \%$ of rated, and temperatures are about $150 \mathrm{~K}$ higher than when the GEMs are functioning (i.e., $\sim 850 \mathrm{~K}$ versus $\sim 700 \mathrm{~K}$ ).

The GEMs can be helpful in three respects. First, they add to the safety margin regarding sodium boiling, and this is a crucial threshold to avoid. Second, if one or more of the pump coastdowns are less than anticipated, e.g., if one of the synchronous machines seizes, then the GEMs could help avoid a potentially serious accident. Third, during an extended unscrammed event, the reactor vessel expands and pulls the control rods out somewhat from the core, and the GEMs help to overcome this delayed reactivity addition, which can be quite significant.

\subsection{3 ULOHS Only}

The event is initiated from full power conditions, as listed in Table 1. The secondary loop heat transfer is arbitrarily terminated, so that all heat generated is retained in the primary vessel. The vessel heat up is assumed to be adiabatic. The EM pumps continue to operate normally, and the plant protection system is assumed to not scram the reactor. As discussed previously, the thermal conductivity used is the reduced case, as shown in Table 3 , in order to account for the uncertainties in the data collected to date and to reflect the fuel's behavior under irradiation. 


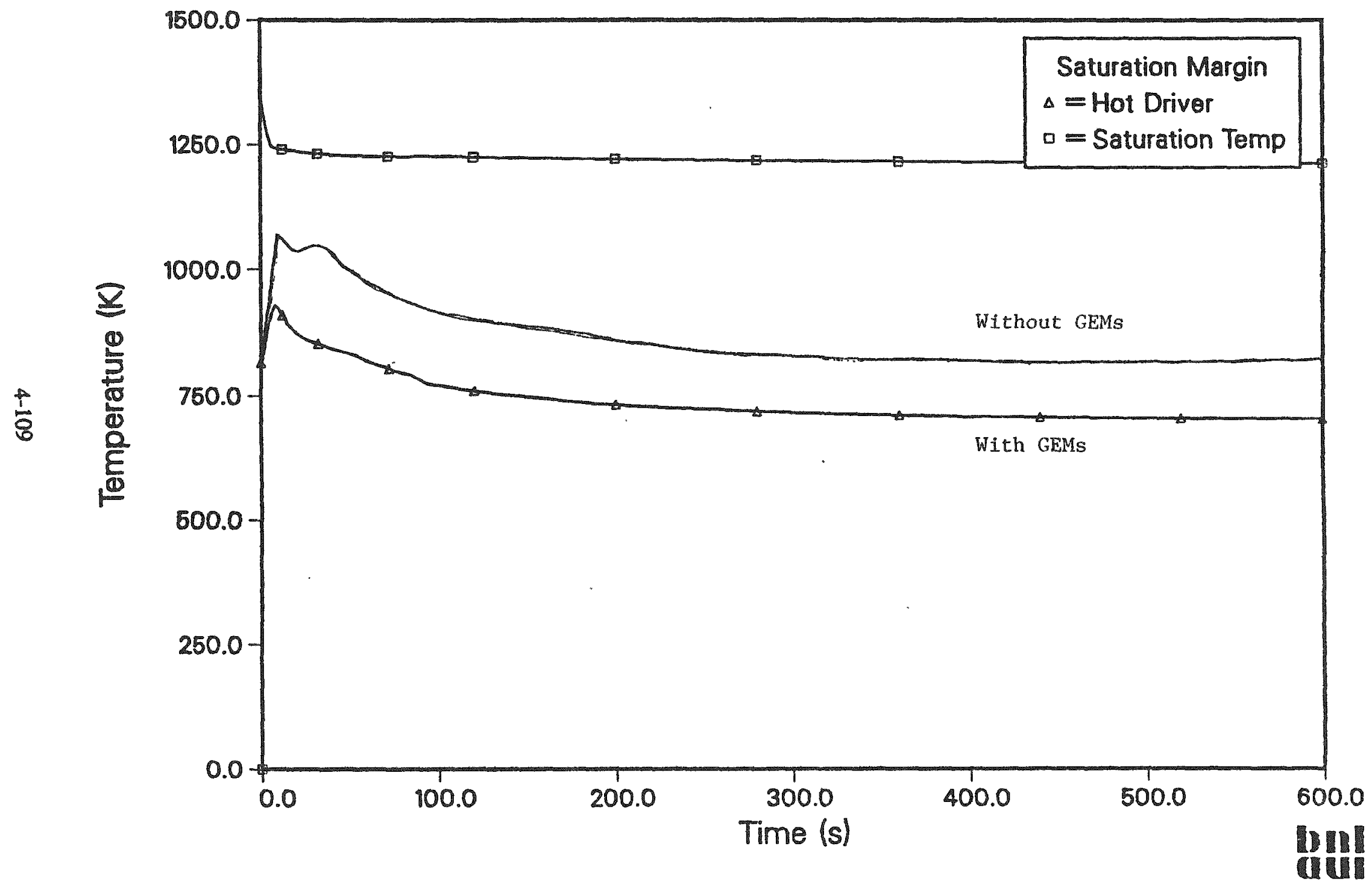

Figure 65. Predicted Margin to Sodium Saturation from SSC for a ULOF With and Without GEMs. 


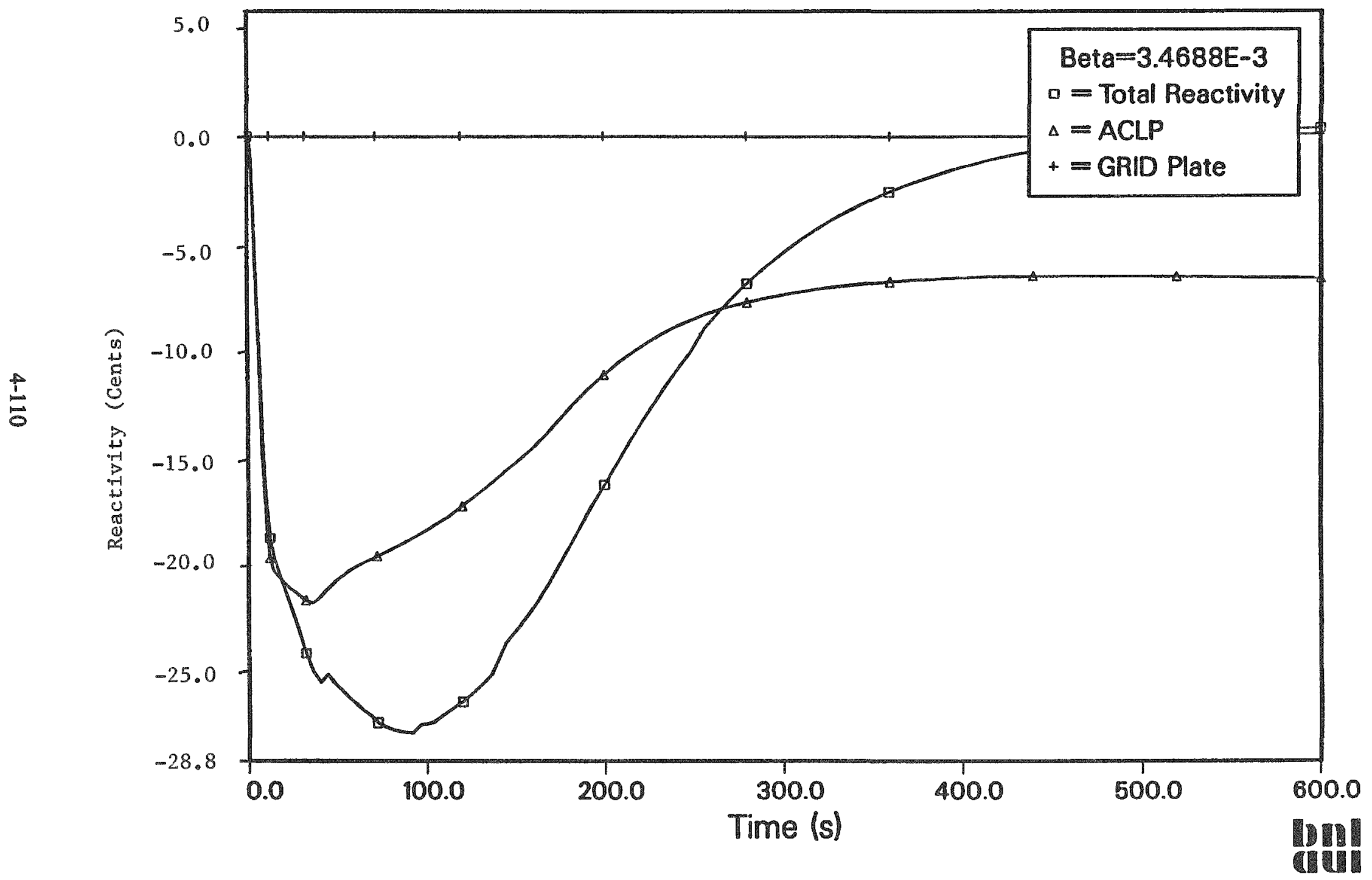

Figure 66. Predicted Total, ACLP, and GRID Plate Reactivity Feedback from SSC for a
ULOF Without GEMs. 


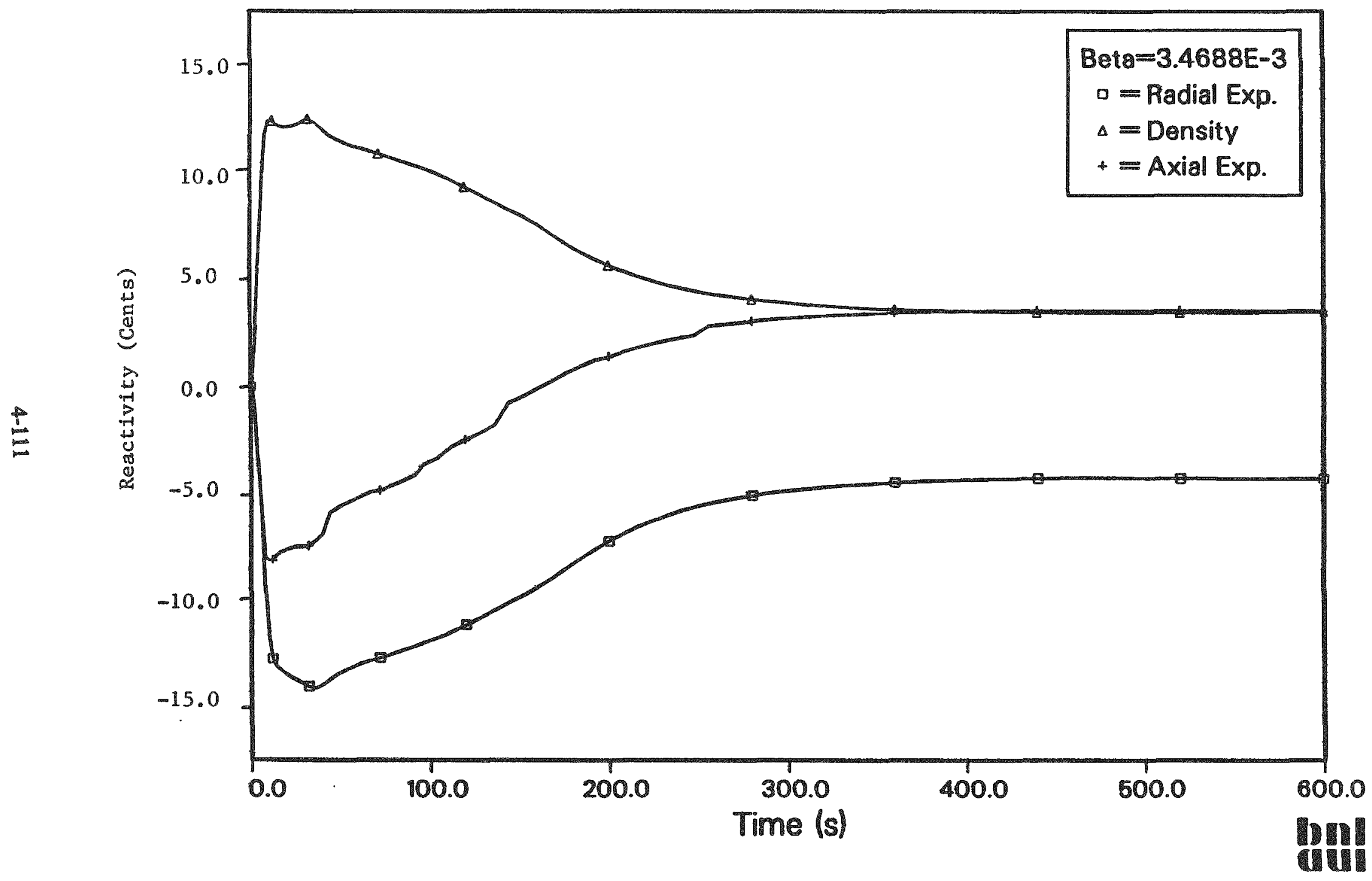

Figure 67. Predicted Radial Expansion, Sodium Density, and Axial Expansion Reactivity Feedback from SSC for a ULOF Without GEMs. 


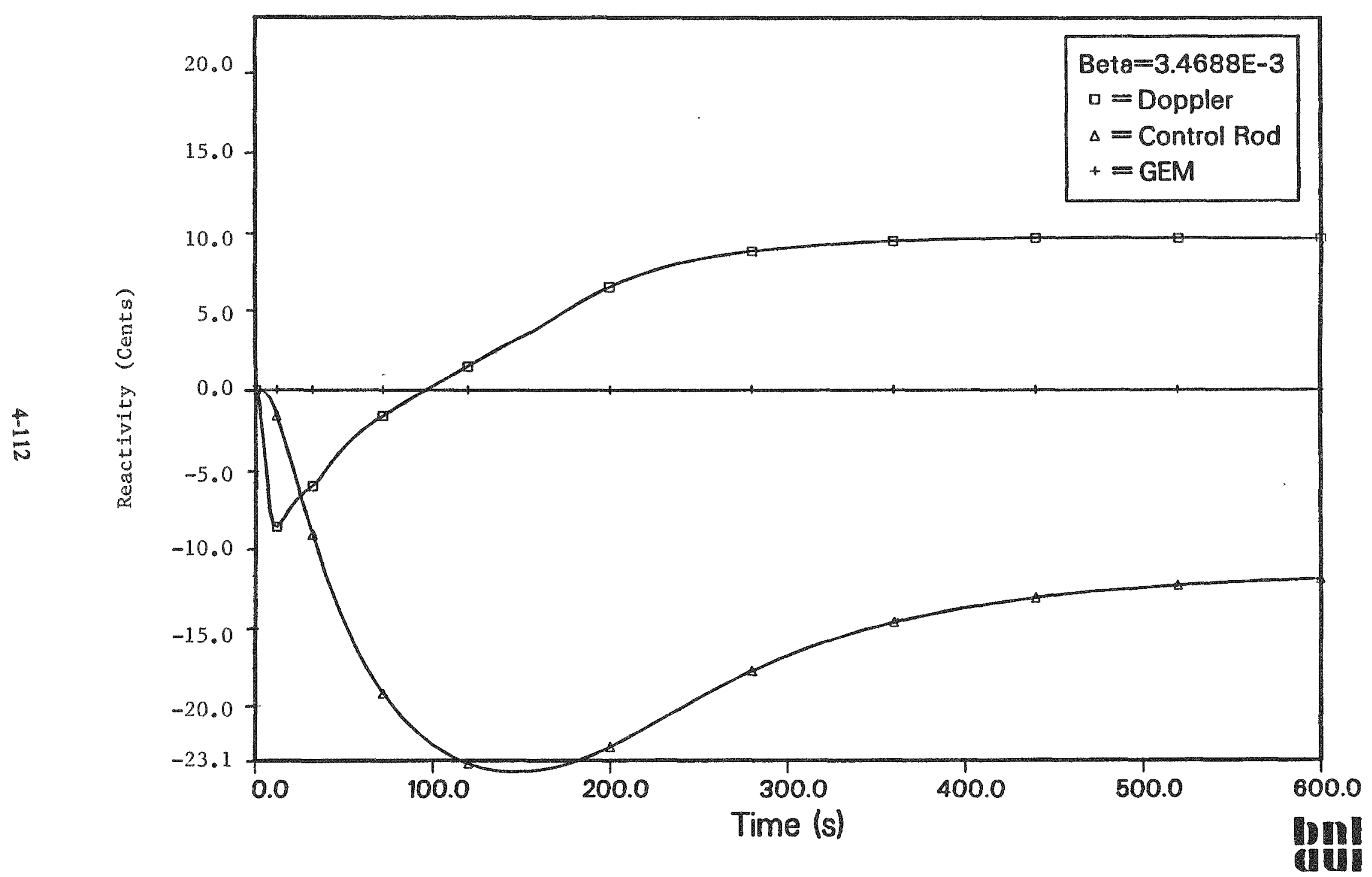

Figure 68. Predicted Doppler, Control Rod Drive Line Expansion, and GEM Reactivity Feedback from SSC for a ULOF Without GEMs. 


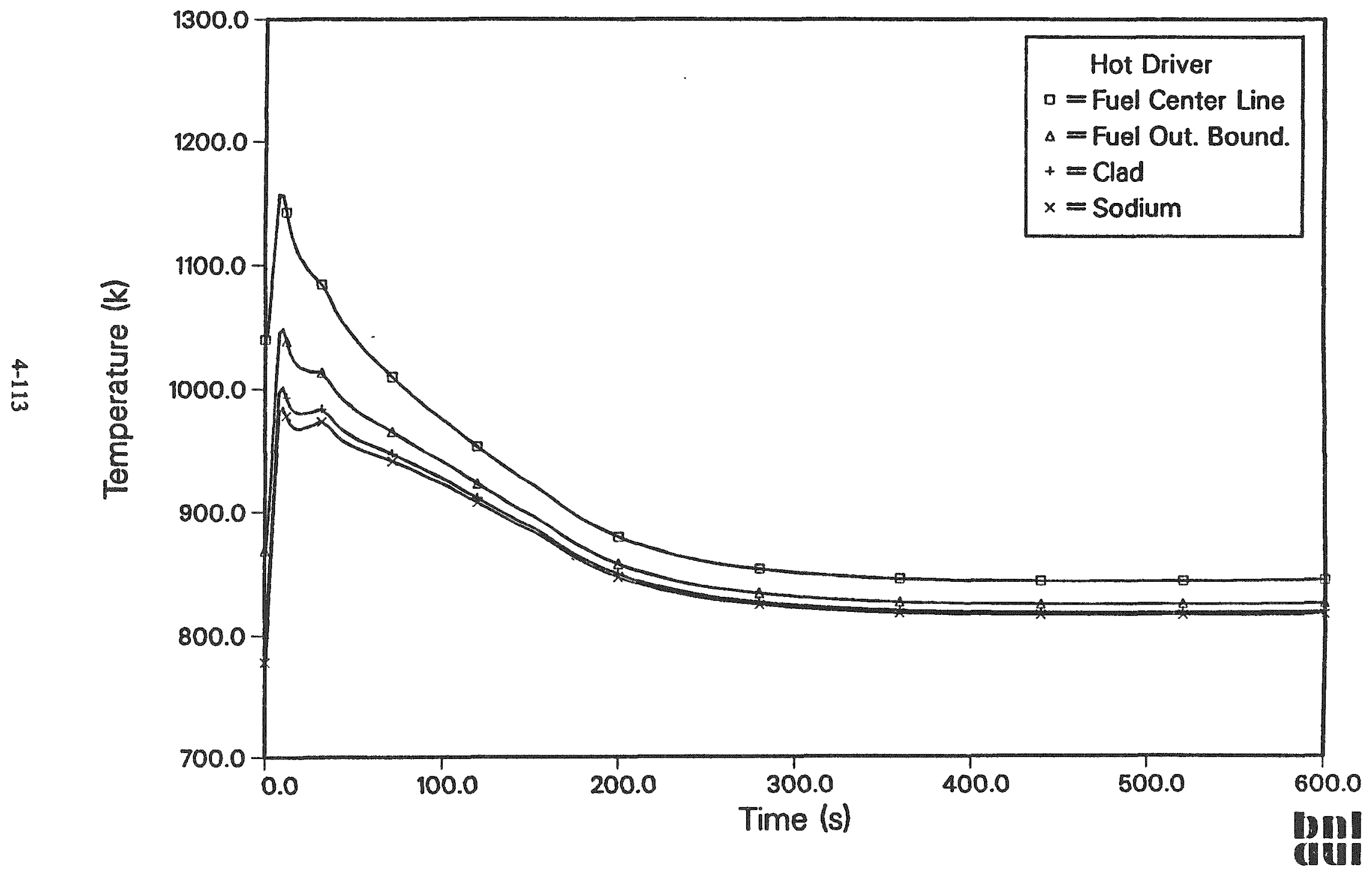

Figure 69. Predicted Fuel Temperature Distribution from SSC for the Second Node from the Top (i.e. 1.122 m- 0.897 m) for a ULOF Without GEMs. 


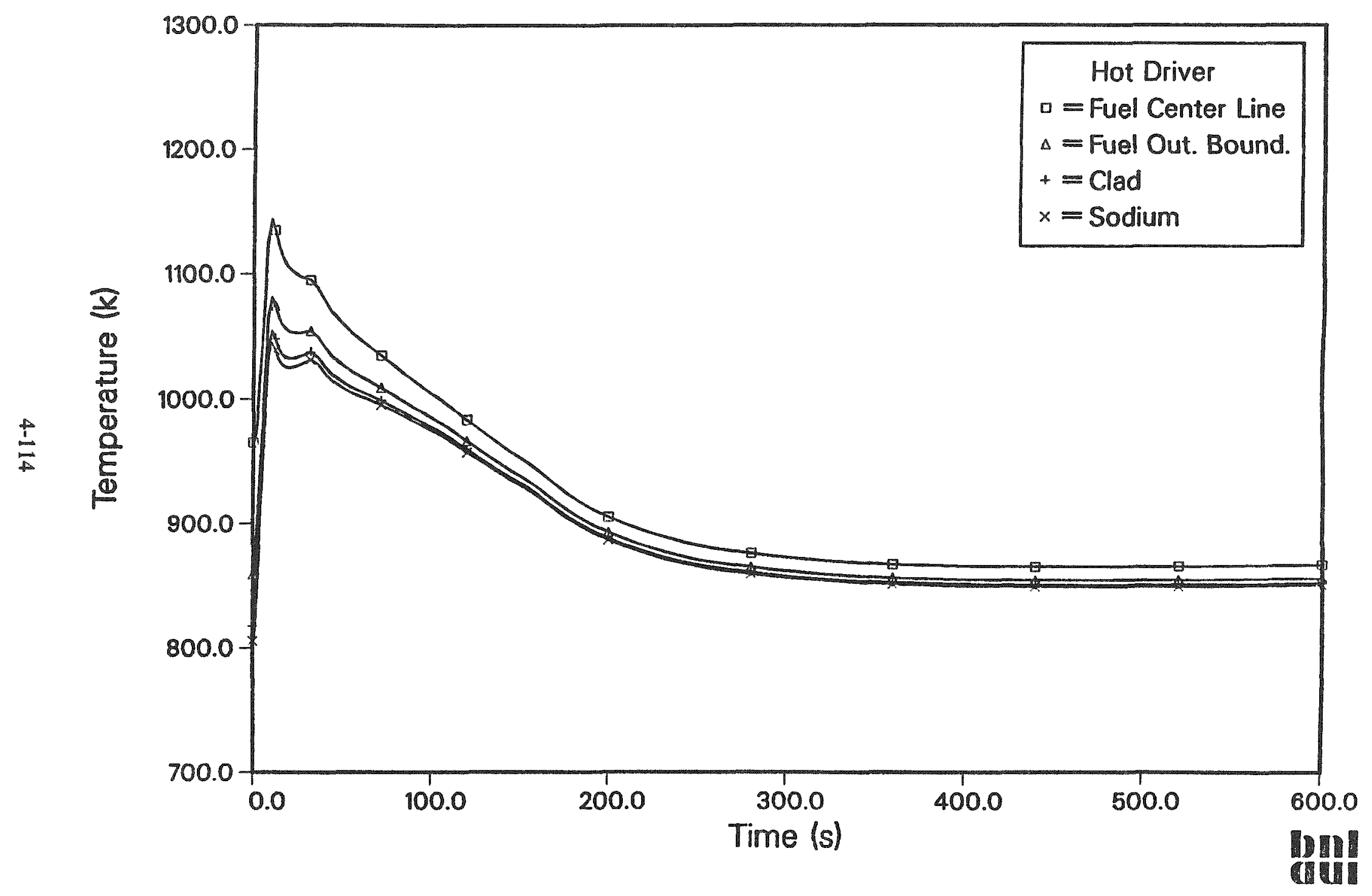

Figure 70. Predicted Fuel Temperature Distribution from SSC for the Top Node (i.e.o $1.346 \mathrm{~m}-1.122 \mathrm{~m}$ ) for a ULOF Without GEMs. 
Within 10 seconds of the IHX being shut off, the temperature of the sodium entering the IHX is the same as that leaving, as seen in Figure 71. The result can be seen in Figure 72, where the power level transitions to a decay heat level by 500 seconds. Figure 73 shows the sodium temperature in the core inlet plenum starting to increase by $80 \mathrm{~s}$. The outlet sodium temperature is shown in Figure 74. The slow heat up of sodium is because of the large sodium pools and the metal mass, giving the primary sodium a big thermal sink.

The resultant neutronic feedbacks are shown in Figures 75 through 77 . Figure 75 shows that the increase in sodium temperature results in the net reactivity dropping down to about $-27 \mathrm{c}$ by $600 \mathrm{~s}$. This is what is left over after overcoming the power defect, since the absolute temperature of the system has increased. The radial expansion, shown in Figure 76, is the dominant feedback in this event, and drives the net feedback, with the grid plate expansion shown to be the largest contributor to the negative feedback. The hot inlet sodium thermally expands the grid plate and reduces the fuel density by spreading the assemblies of the core, causing more fast neutron leakage. These higher temperatures also increase the axial thermal expansion feedback, contributing about -8 cents. The higher temperatures increase the positive feedback from the sodium density to about 14 cents. Figure 77 shows that the Doppler feedback is worth only about -5 cents. The control rod drive line expansion has a maximum reactivity worth of only $-7 \mathrm{c}$ by $200 \mathrm{~s}$. By $200 \mathrm{~s}$, the rods are already being withdrawn due to the vessel expansion, which pulls the cantilevered rods back out of the core once the vessel expansion out paces the control rod drive line expansion. However, over the long term, the grid plate expansion and other thermal expansion feedbacks largely counteract the effect.

The temperatures in the hot driver are shown in Figures 78 and 79. The temperature decreases after about 75 seconds. This figure is representative of all the mid-core temperatures. Some of the fuel centerline temperatures below the core center increase because of the increase in the sodium inlet temperature. Since the reactor power transitions to decay heat levels, the fuel temperatures are not a concern for this event. Finally, Figure 80 shows the margin to sodium voiding to be about $560 \mathrm{~K}$, which is quite large and not a reason for concern.

Thus, the ULOHS event does not appear to pose a significant challenge to the PRISM passive shutdown. The peak fuel temperatures all decrease, and show no fuel damage during the first $600 \mathrm{~s}$. The only concern might be the extent of this transient and any impact on the service limits.

\subsection{Comparing the ULOF and ULOHS Cases}

The peak fuel, clad, and sodium temperatures for the various unscrammed loss of flow and/or heat sink, along with key temperature limits are shown in Figure 81. GE's results for Event 2' are represented in the first set of bars, which directly correspond to those included in Figure 16. Peak temperatures from the equivalent BNL calculations are shown in the second set of bars. The difference in peak fuel temperatures remains unresolved. The peak fuel temperatures in the BNL calculation occur at the beginning of the transient, because tripping of the pumps triggers a quick response from the GEMs, which brings the power down before the system can begin to heat up. It is unclear to us why the fuel temperatures increase in the applicant's analysis. With respect to the peak clad and coolant temperatures, both analyses indicates similar temperature increases, which could lead to some cladding damage, depending on how the sparse data available is interpreted (see Section 4.16.3.2).

The BNL results for the ULOF event, with and without the GEMs functioning, are represented by the 3rd and 4th sets of bars. Obviously, the peak temperatures with the GEMs functioning properly pose little concern. However, without the GEMs, there could be significant cladding damage. 


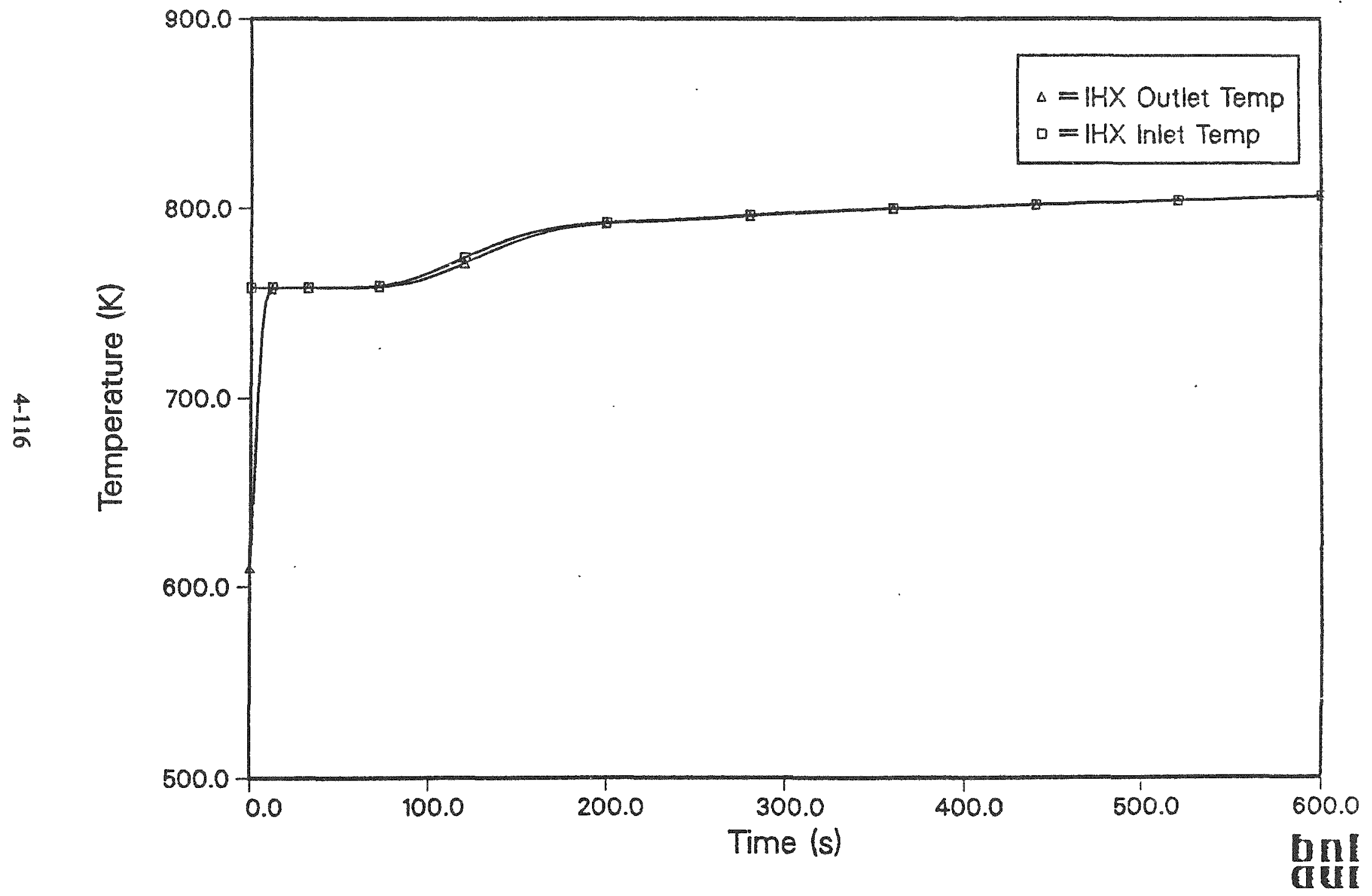

Figure 71. Predicted Inlet and Outlet IHX Temperatures from SSC for a ULOHS. 


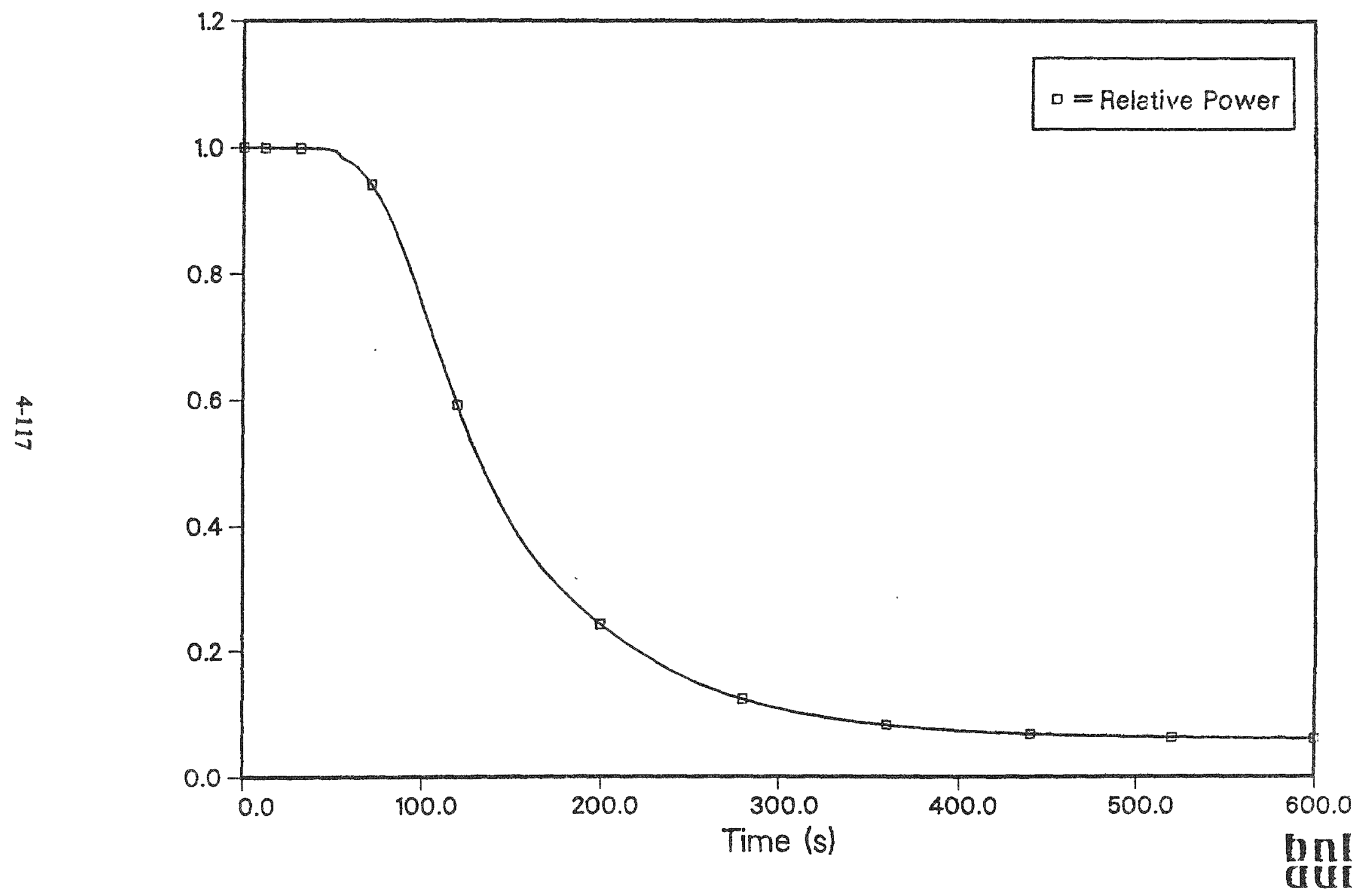

Figure 72. Predicted Relative Power from SSC for a ULOHS. 


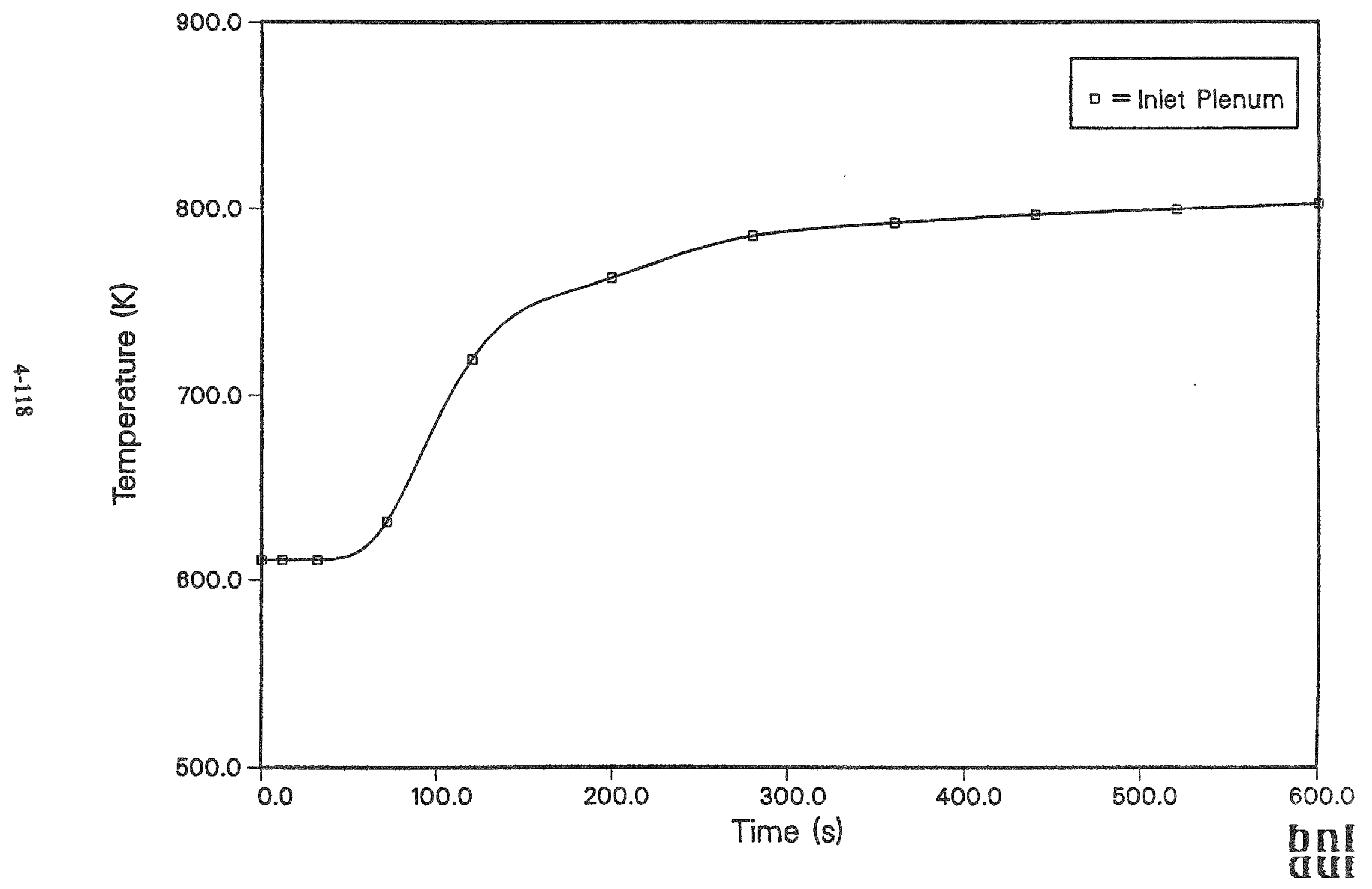

Figure 73. Predicted Core Inlet Plenum Bulk Average Temperature from SSC for a ULOHS. 


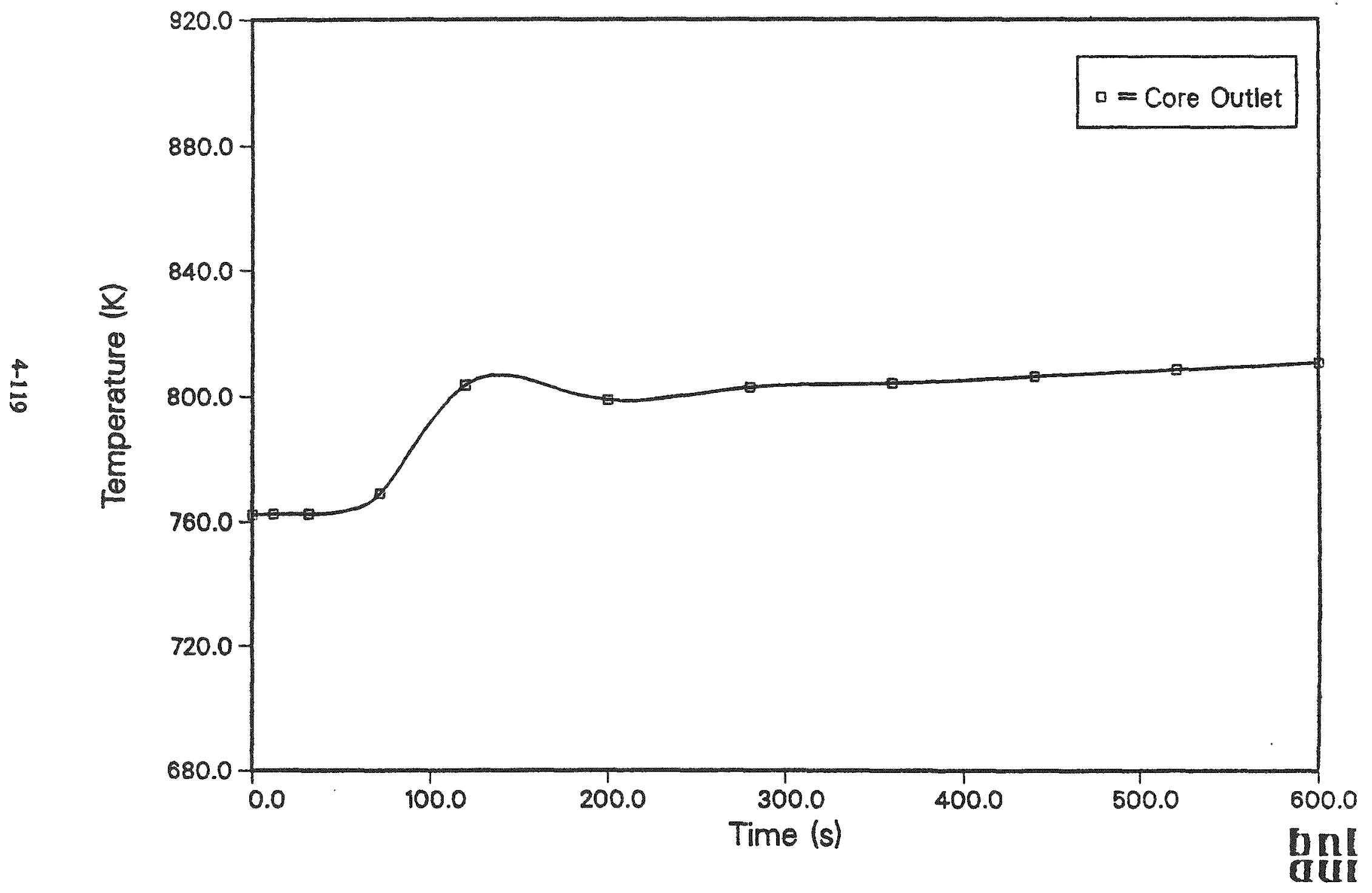

Figure 74. Predicted Average Core Outlet Sodium Temperature from SSC for a ULOHS. 


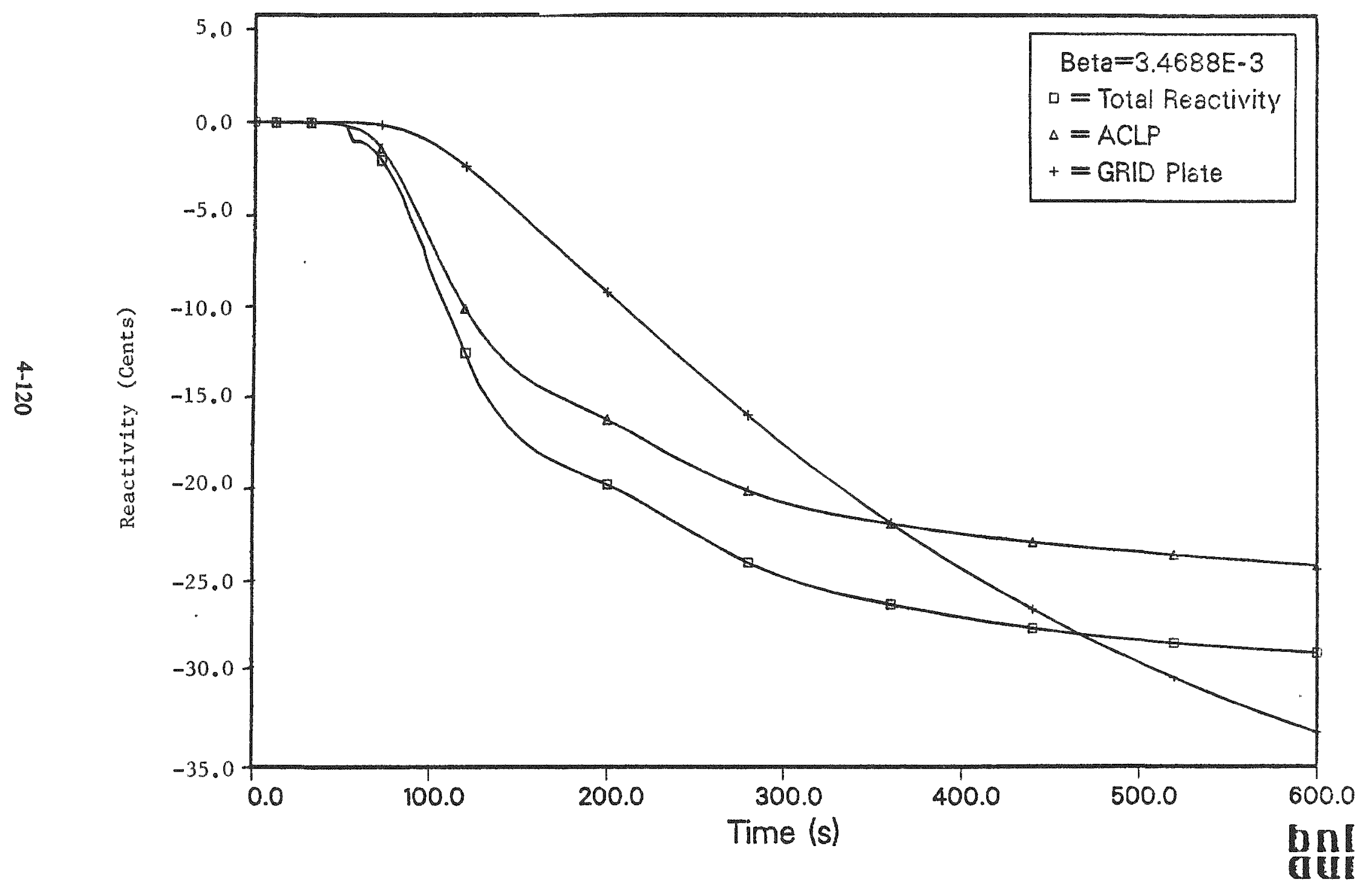

Figure 75. Predicted Total, ACLP, and GRID Plate Reactivity Feedback from SSC for a ULOHS. 


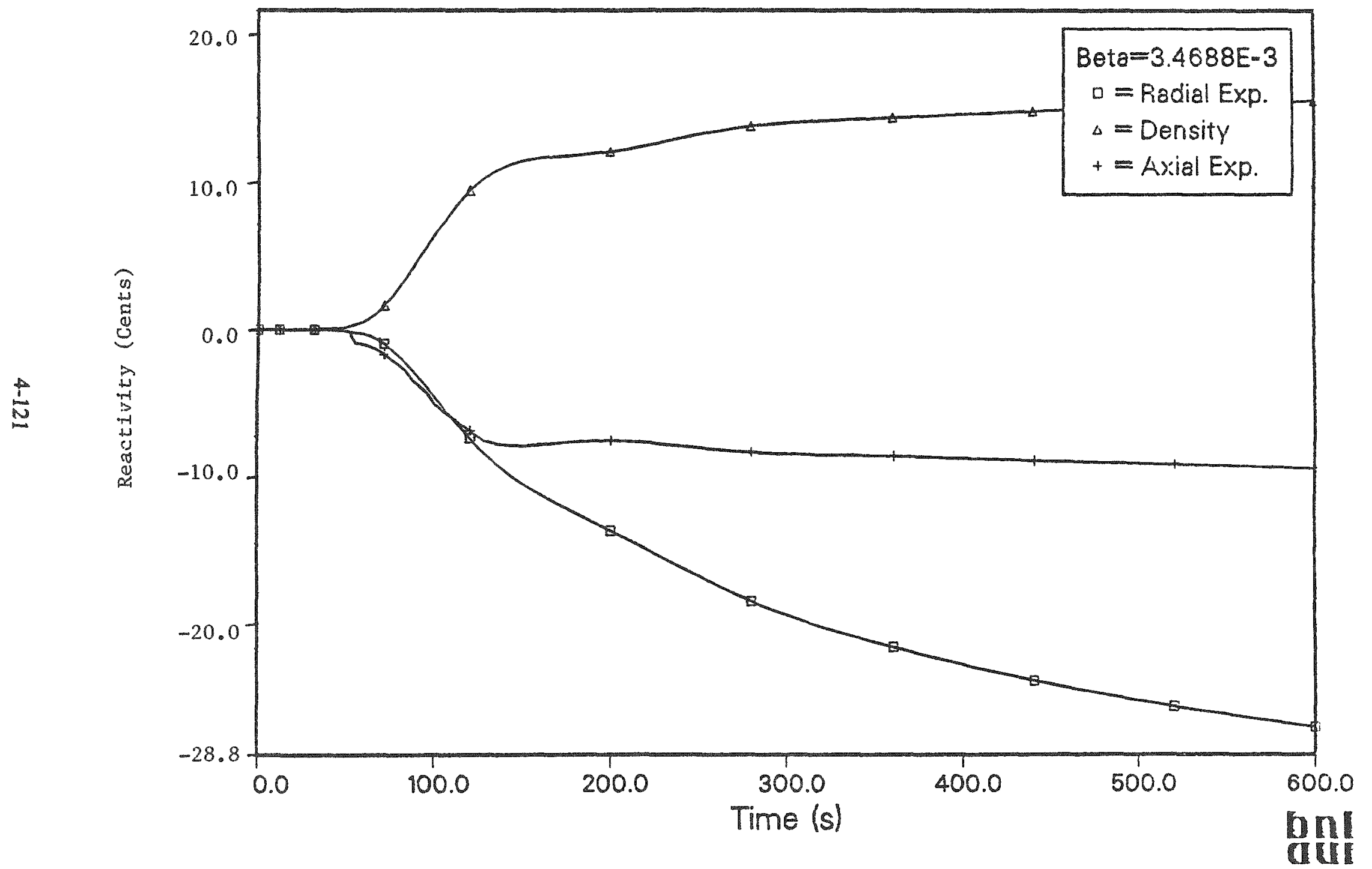

Figure 76. Predicted Radial Expansion, Sodium Density, and Axial Expansion Reactivity Feedback from SSC for a ULOHS. 


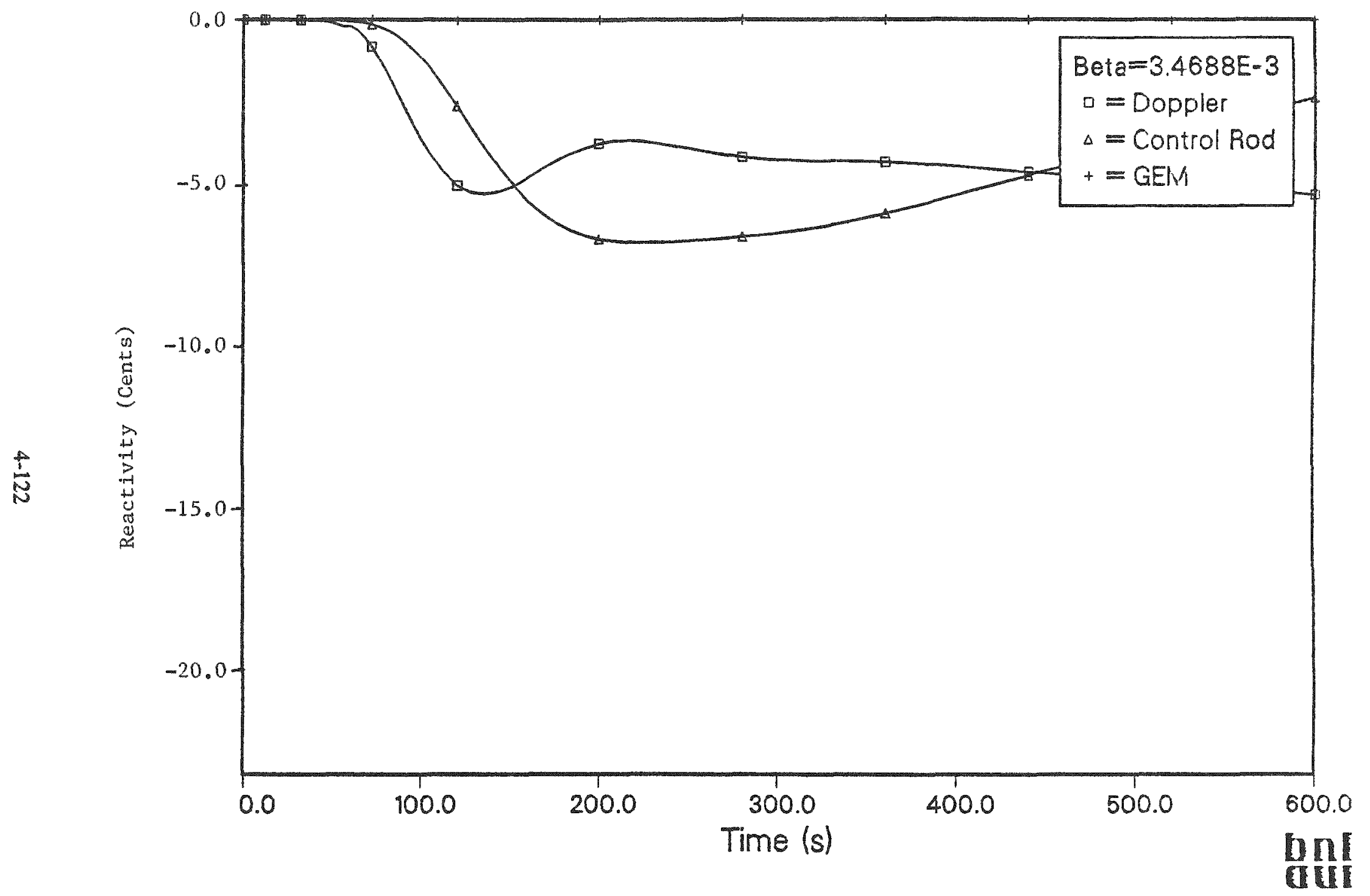

Figure 77. Predicted Doppler, Control Rod Expansion, and GEM Reactivity Feedback from SSC for a ULOHS. 


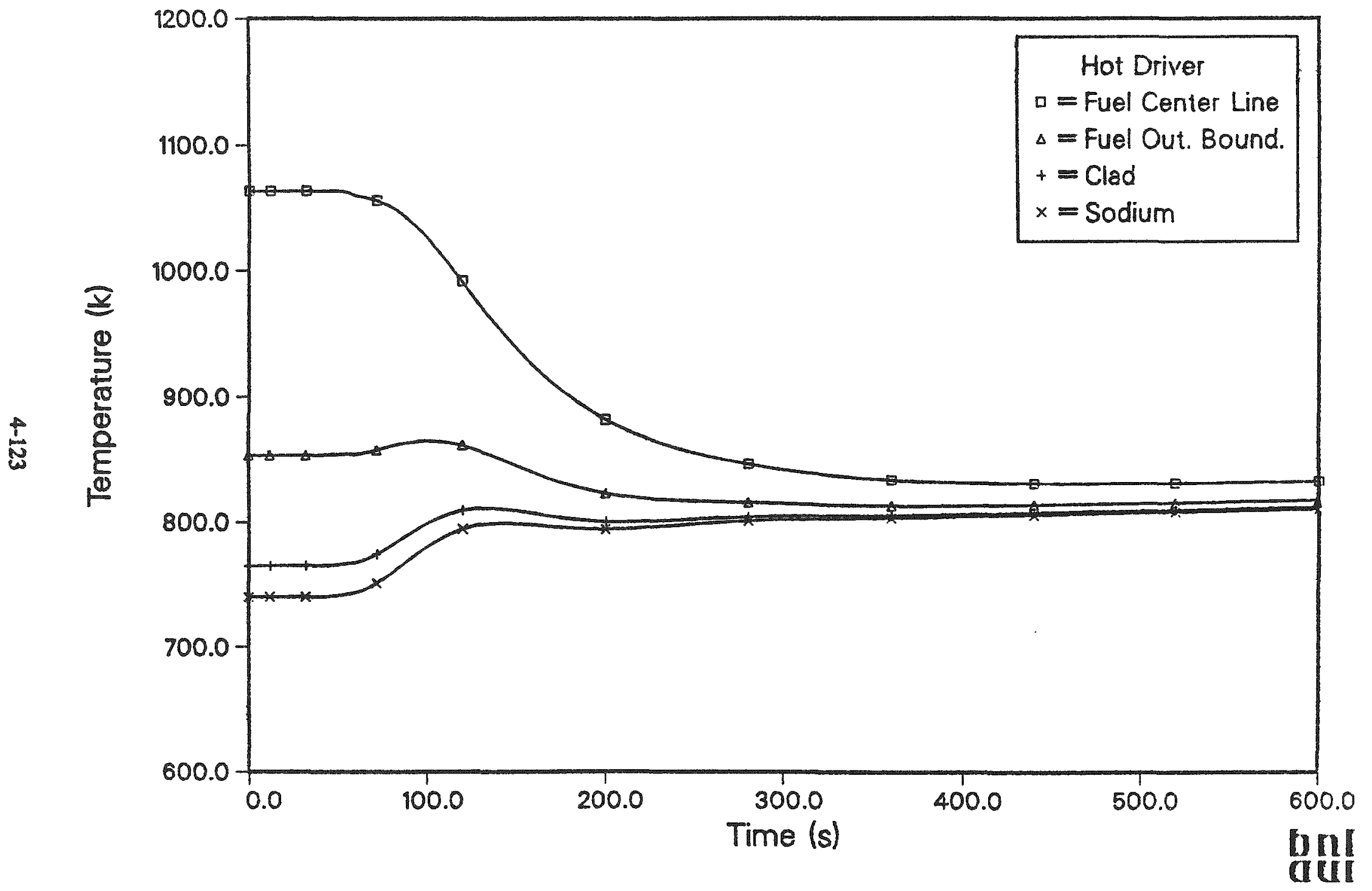

Figure 78. Predicted Fuel Temperature Distribution from SSC for the Third Node from the Top (i.e., $0.897 \mathrm{~m}-0.673 \mathrm{~m}$ ) for a ULOHS. 


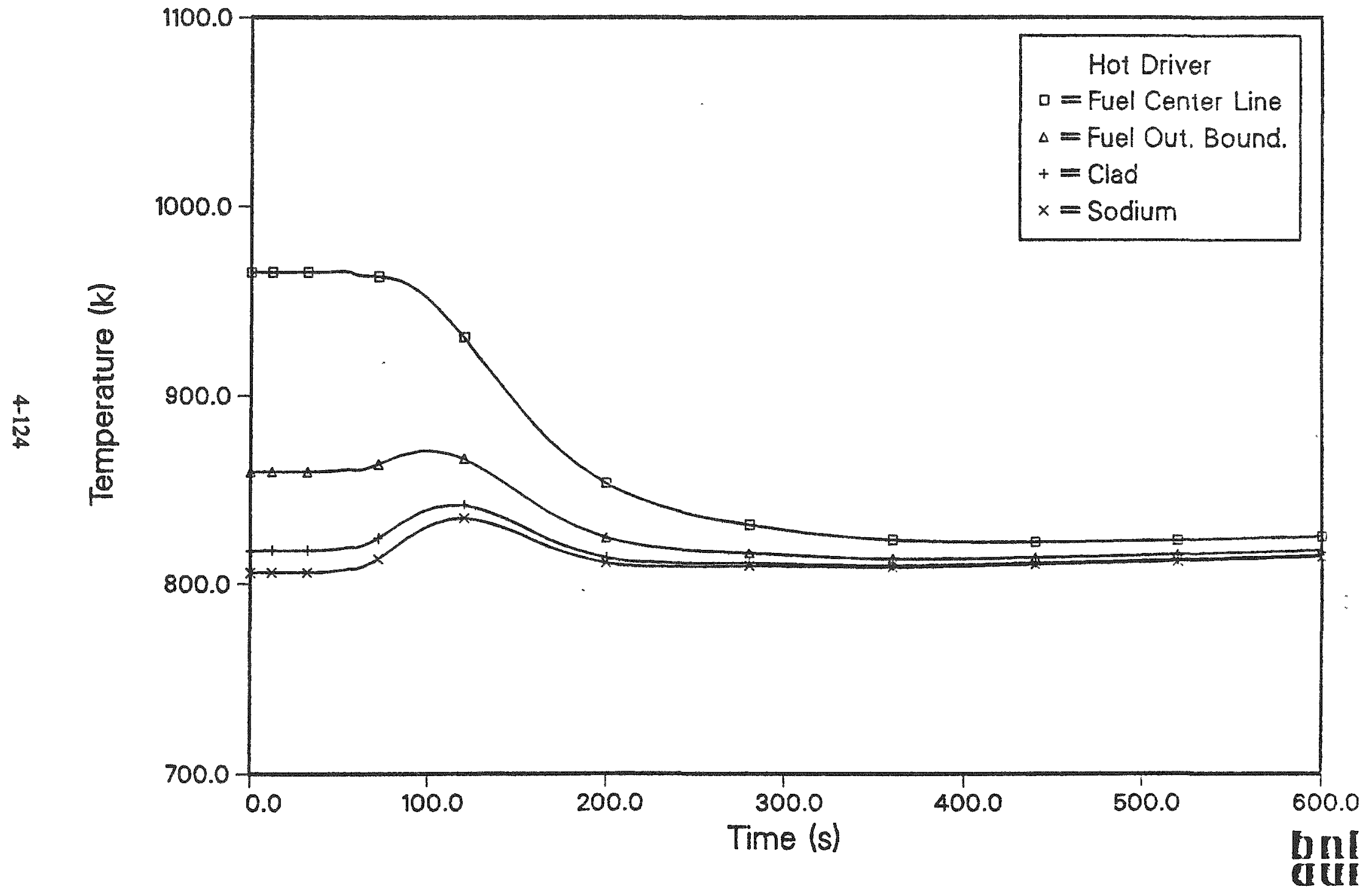

Figure 79. Predicted Fuel Temperature Distribution from SSC for the TOP Node (i.e., $1.346 \mathrm{~m}-1.122 \mathrm{~m}$ ) for a ULOHS. 


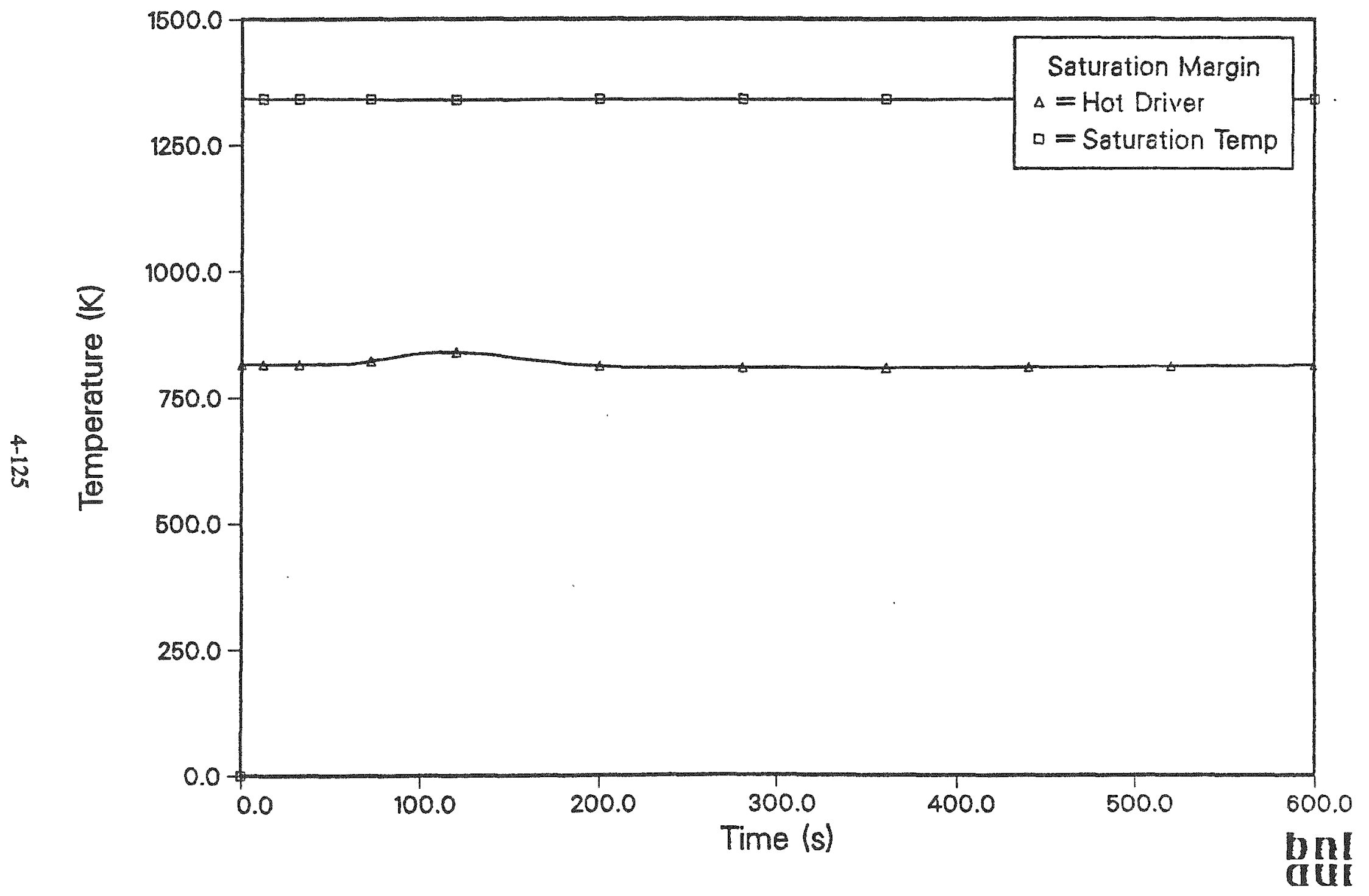

Figure 80. Predicted Margin to Sodium Saturation from SSC for a ULOHS. 


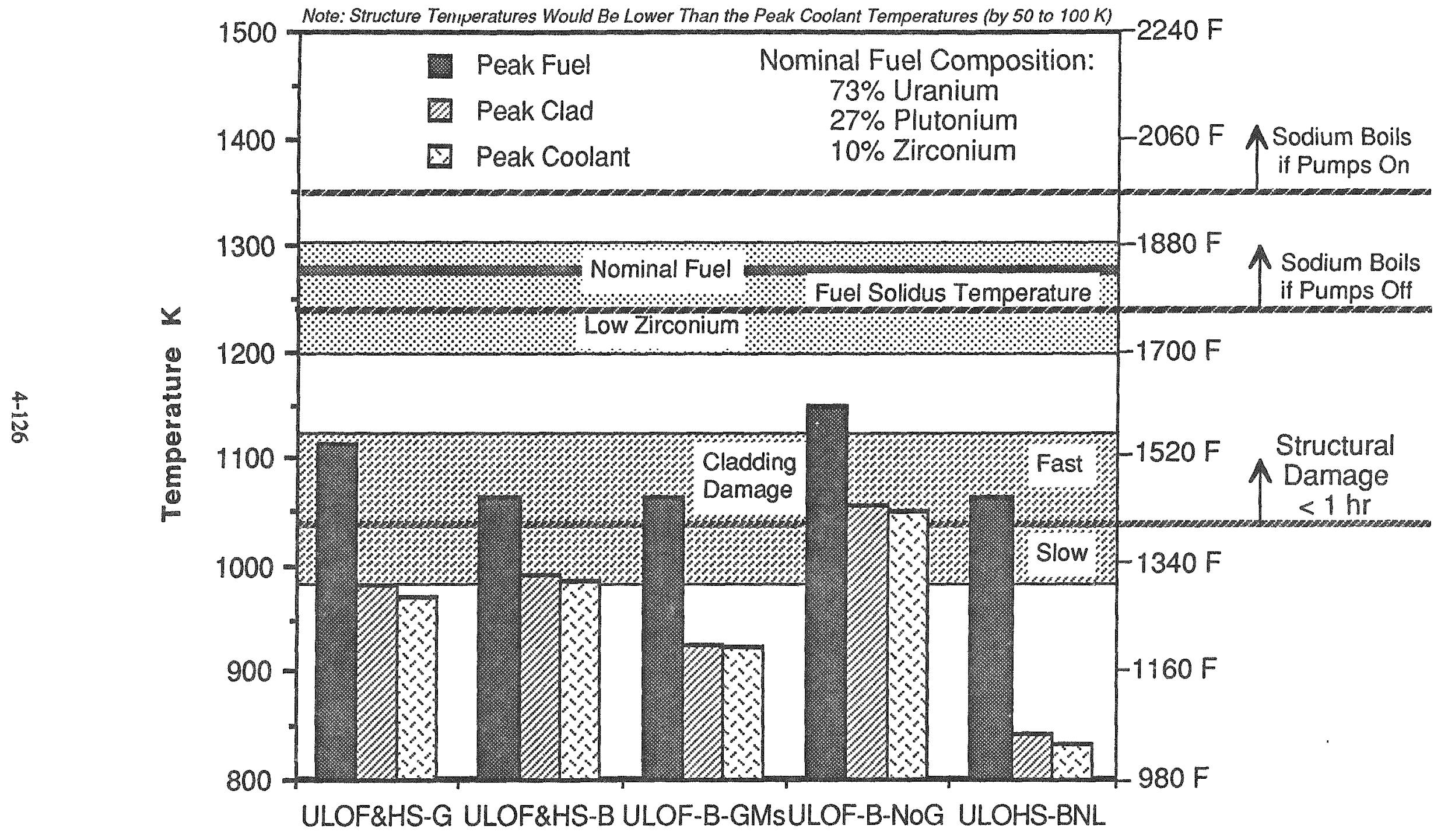

Unscrammed Loss of Flow, Heat Sink Events

Figure 81. Peak Fuel, Clad, and Sodium Temperatures for Various Unscrammed Loss of Flow and/or Loss of Heat Sink Events. 
The 5th case, the unscrammed loss of heat sink does not appear to be a significant safety concern, unless it continues indefinitely. Certainly, the peak temperatures are very modest over the near term, as indicated in Figure 81.

\subsection{LOHS Events with Partial Blockage of RVACS Ducting}

Because RVACS is the only safety-grade heat removal system for PRISM, it has been closely scrutinized for potential failure modes. While some degradation in system performance is possible (heat transfer surfaces may change) and some partial failures (reactor vessel leaks, for instance) are conceivable, the failure mode that seems most significant would be a blockage of the air-flow ducting. However, these 4 independent air ducts are very large and difficult to fully block, except via a massive earthquake or an extremely thorough act of sabotage.

Because RVACS is such a crucial system, the NRC staff felt that some degree of failure should be assumed for further analysis. (This failure is in addition to a loss of normal cooling and loss of ACS, which are not safety systems, but have excellent natural cooling capabilities regardless). Initially, a very lengthy adiabatic heat-up was imposed, which lead to predictions of severe damage about 1 day into the event. However, the definition of this event was somewhat arbitrary and pre-dated some revisions in the applicant's positions regarding containment and the safety function of the operator (thereby reducing the likelihood and consequences of such an unlikely event).

\subsection{75\% Blockage of Air-Flow Ducting}

The independent analysis for this event is discussed in Section 4.9.4, with the peak reactor outlet sodium temperatures as shown in Figure 82. Both GE's results and BNL's results indicate that system temperatures peak out around $925 \mathrm{~K}$ (1205 F) about 40 hours into the event. The principle concern here would be for the system structures, as ASME "C" limits are around $922 \mathrm{~K}$. However, "C" limits are primarily an investment concern, as if these temperatures are exceeded the process of restarting the unit would involve a detailed re-evaluation by safety authorities. In addition, the cladding temperatures for this event (close to the sodium temperatures) could cause significant damage to the fuel loading.

\subsection{Full blockage of Ducting for 12 Hours, Then $25 \%$ Re-Opened}

The independent analysis for this event is discussed in Section 4.9.5, with the peak sodium temperatures included in Figure 82. Both GE's results and BNL's results indicate peak temperatures in the range of $975 \mathrm{~K}(1296 \mathrm{~F})$, coming around 25 hours into the event. These peak temperatures are near ASME "D" limits, and some damage to the structures may result. There may also be some localized cladding damage in the hotter portions of the reactor.

The first 12 hours of this event involve an adiabatic heat-up of the primary system, with temperatures climbing more than $200 \mathrm{~K}(360 \mathrm{~F})$ during that time. If the adiabatic heat-up were to continue, severe damage would occur before the end of 24 hours. However, the likelihood of the PRISM system ever experiencing an adiabatic heat-up, given that all three heat removal systems function well under natural circulation, seems extremely small. Further, twelve hours is a lengthy period for someone to restore partial functioning of one or more of the heat removal systems. Therefore, generally speaking, the adiabatic heat-up category of events seem unlikely to dominate the risks in operating a PRISM unit. 


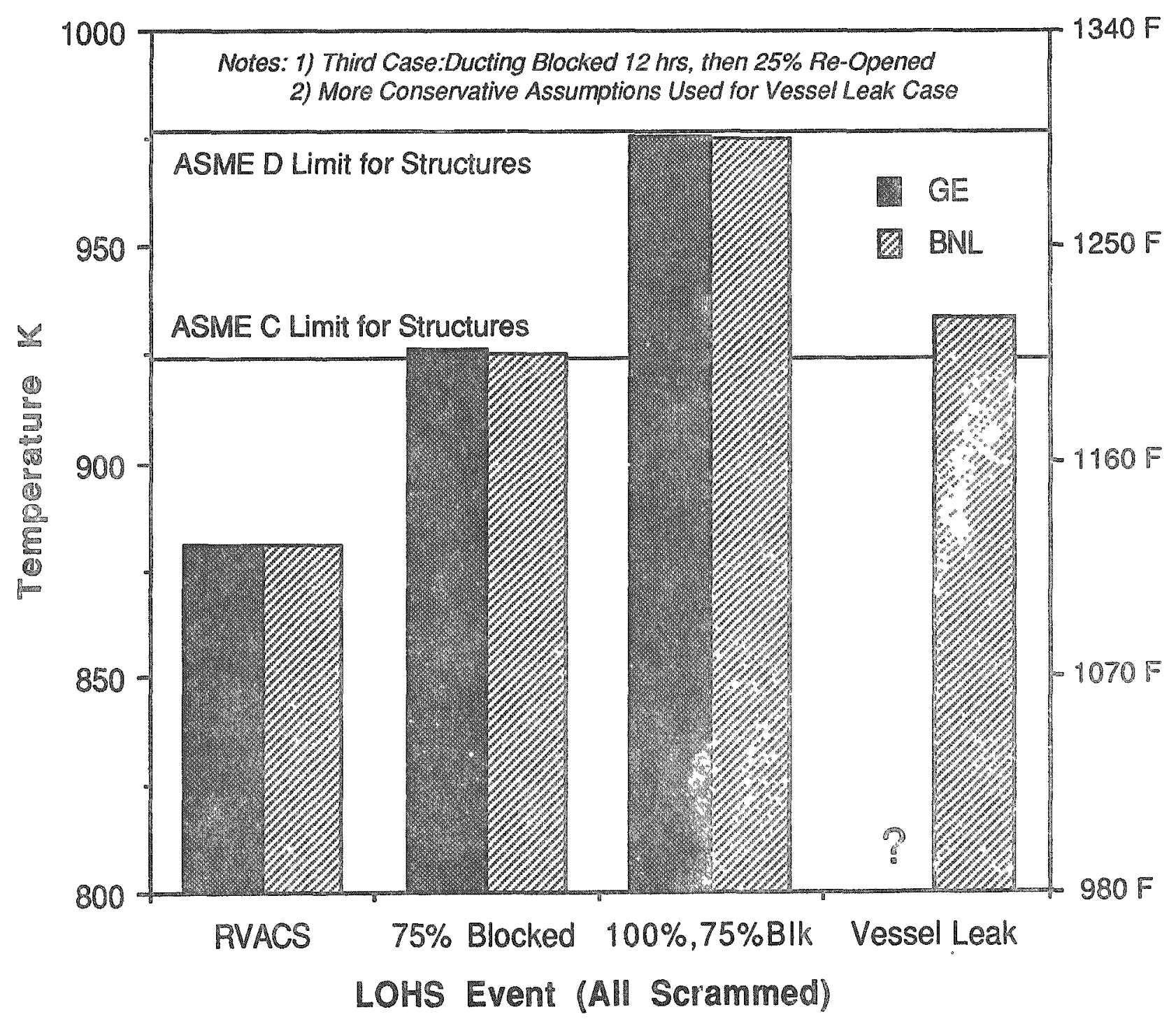

Figure 82. Peak Reactor Outlet Sodium Temperatures for Scrammed LOHS (RVACS-Only) Events 


\subsection{ULOF Events With Coastdown Failures}

As an EM pump has virtually no inertia, it was necessary for GE to use synchronous machines to provide an artificial coastdown. These machines, which are little more than flywheels coupled with motor-generator units, are operated continuously so that if there is a power loss or other malfunction then there will be a resultant coastdown. As the synchronous machine is coasting down, the rotational energy is tapped and diverted to the EM pumps, which experience a gradual, pre-programmed reduction in power.

Because the passive reactor shutdown requires some time to bring the power down, the absence of pump coastdowns can be a major safety concern. Therefore the synchronous machines and the cables are crucial safety components. The designer's decision to move these machines into the seismically isolated zone is believed to be a major improvement. Further, the addition of the GEMs has been very helpful, as the large and rapid negative reactivity feedback that results when the pumps are tripped really boosts the effectiveness of the passive shutdown.

Even though the designer's modifications in this area have addressed some concerns, possible pump coastdown failure modes remain an area of concern. In this section we cover three cases: the loss of one coastdown during a ULOF event, the instantaneous loss of one pump at full power, and the instantaneous loss of all pumps at full power. It is emphasized that the last case is thought to be exceedingly unlikely, and is considered here mostly because of the GEMs, as there was some chance they might make this event acceptable (unfortunately, they did not).

\subsection{ULOF on 3 of 4 Coastdowns}

In this case, we assume a normal ULOF event with one pump coastdown missing entirely. This is bounding event 4, so our calculations can be compared against those provided by the applicant in Appendix $\mathbf{G}$.

Analysis of this event was complicated by our need to calculate the sodium flow rate through the reactor power using the MINET Code, and to calculate the reactor power using the SSC Code. Since the reactor power level and the sodium flow rate are closely coupled, a couple of passes were needed to assure the two calculations were consistent.

In the MINET modeling, the pumps were represented individually, using the fairly detailed pump head and torque curves provided by GE. Some of the complexity is caused by the stoppage of one pump, which creates an open pipe-like pathway for the sodium to short-circuit back to the inlet of the other pumps. The results can be observed in Figures 83-86. Normally, the flow through each pump quickly drops from $630 \mathrm{~kg} / \mathrm{sec}$. to about $300 \mathrm{~kg} / \mathrm{sec}$, and then coasts down, as shown in Figure 83. Instead, the flow per pump goes to about $500 \mathrm{~kg} / \mathrm{sec}$., and the coastdown from there is more protracted, as shown in Figure 84. These changes are caused by the flow reversing through the failed pump, as shown in Figure 85 . With that line open, the circuit flow resistance is sharply reduced, leading to the surging in the pumps that are coasting down, and the reduced torque that causes the coastdown to be stretched out. As a result the coastdown of sodium flow rate through the reactor, shown in Figure 86, is not nearly as severe as one might anticipate.

The flow coastdown predicted by MINET was used to drive the SSC calculations. The calculated power from the SSC prediction is shown in Figure 87. The reasons for the fast power reduction are shown in Figures 88 through 90 , which include the various reactivity feedbacks at work. In this case, the reactivity from the GEMs, which is shown in Figure 90, dominates the others, especially over the 


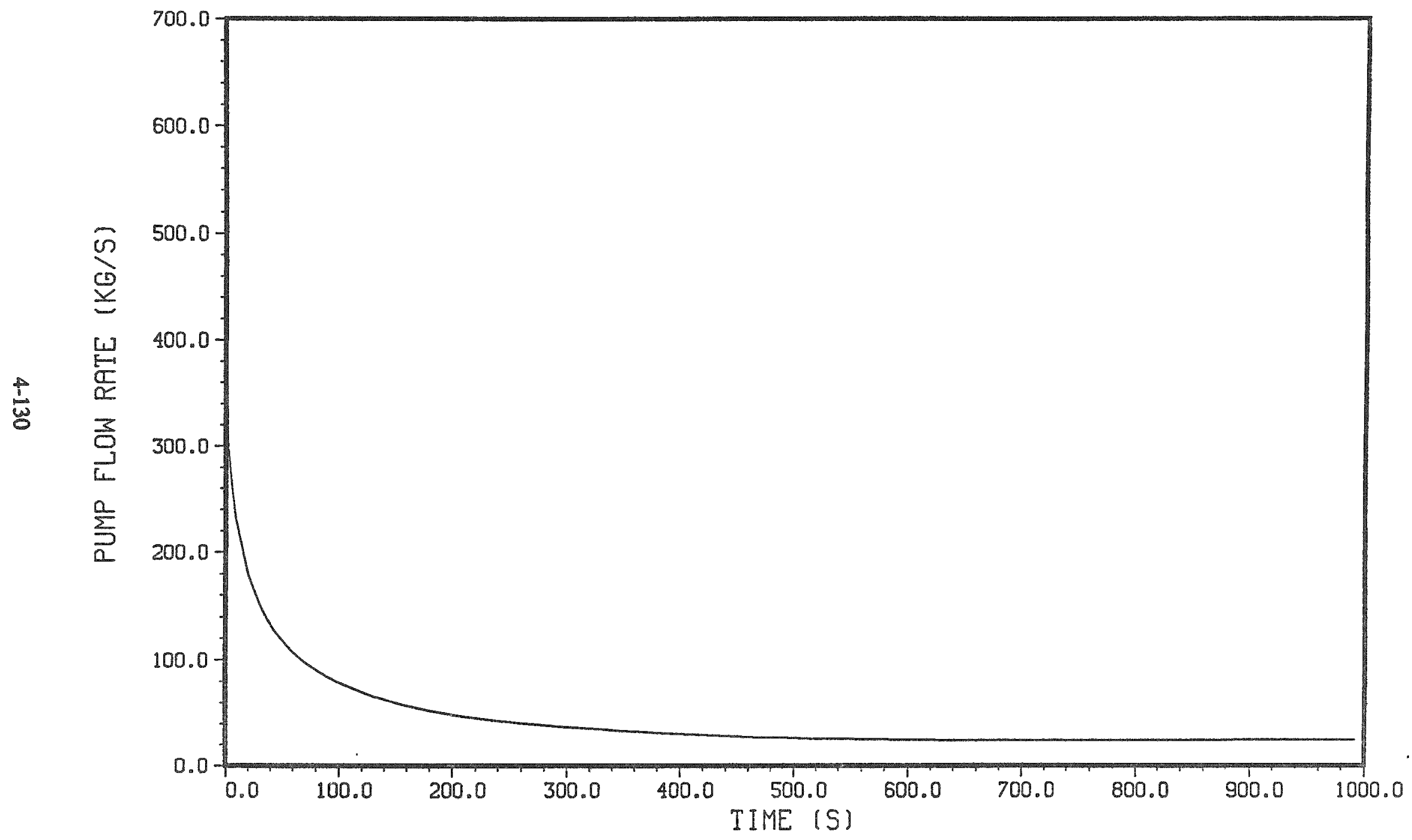

Figure 83. Predicted Pump Flow Rate from MINET for a ULOF/LOHS with 4 EM Pumps. 


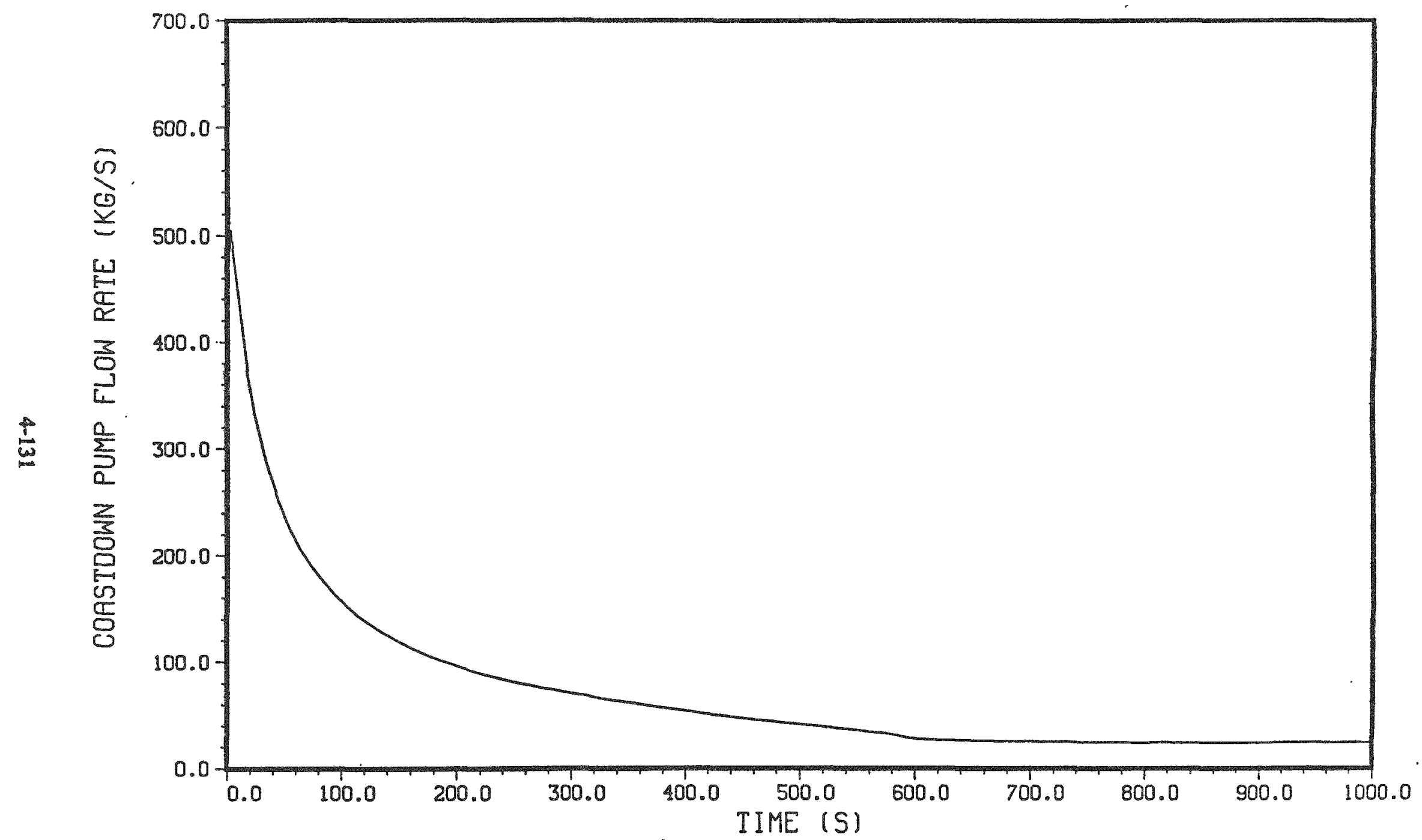
Figure 84. Predicted Pump Flow from MINET for a ULOF/LOHS with a Pump Seizure and 3
Pumps Coastdown. 


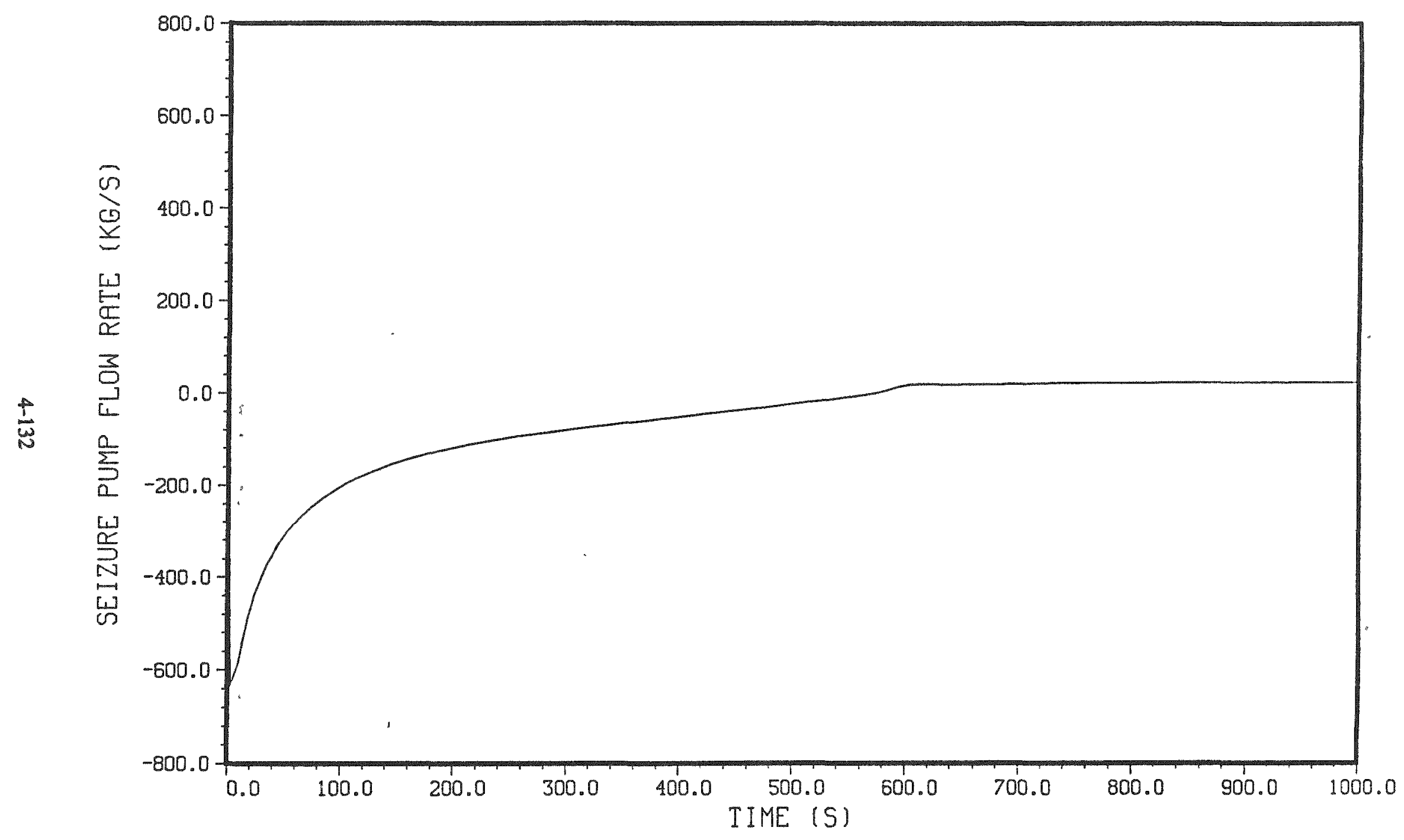

Figure 85. Predicted Flow in a Seized EM Pump for a ULOF/LOHS with Coastdown from 3 Pumps. 


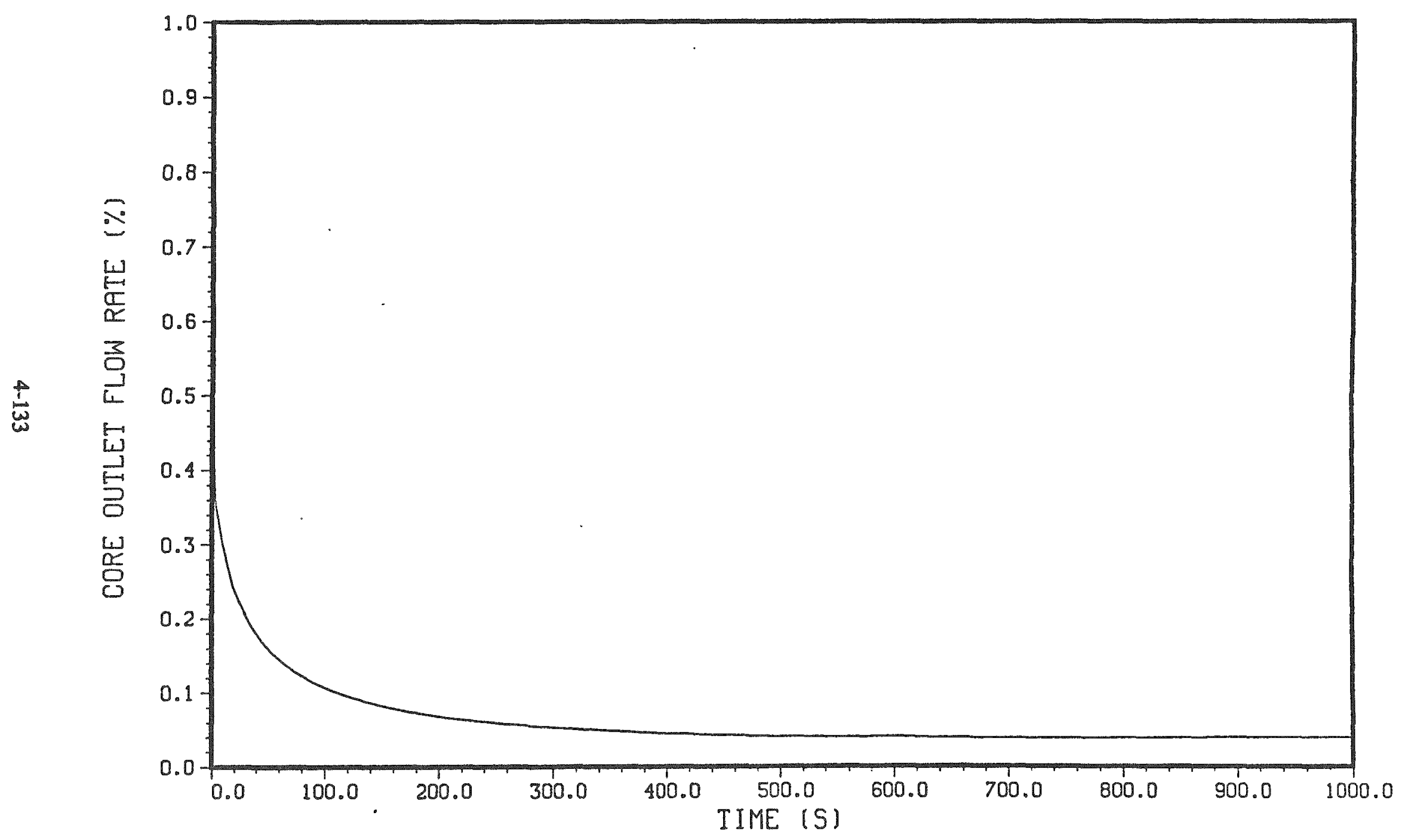

Figure 86. Predicted Core Outlet Flow from MINET for a ULOF/LOHS with 1 Pump Seizure and 3 Pump Coastdown. 


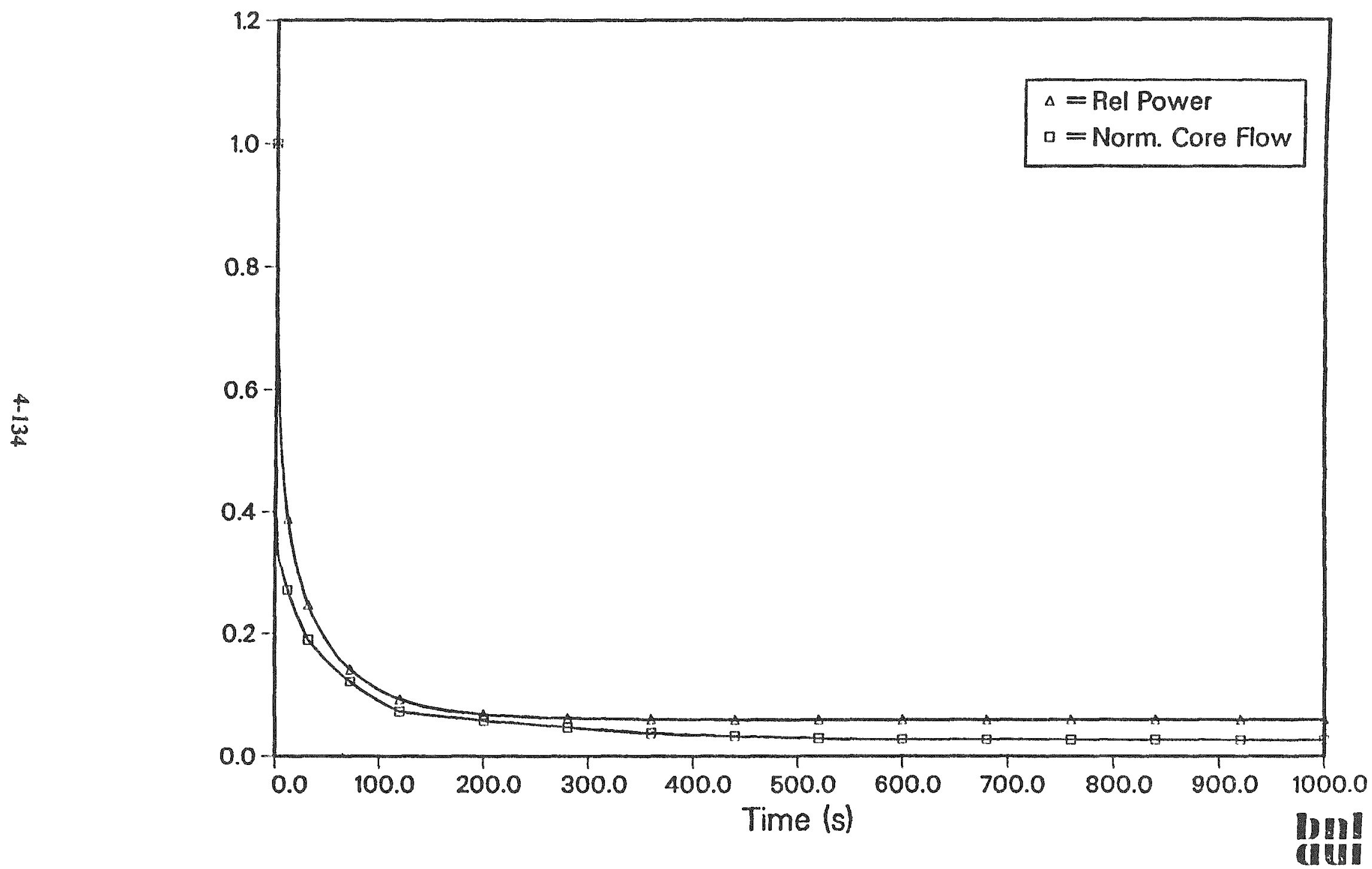

Figure 87. Predicted Relative Power and Normalized Core Flow from SSC for a ULOF with 1 Pump Seized. 


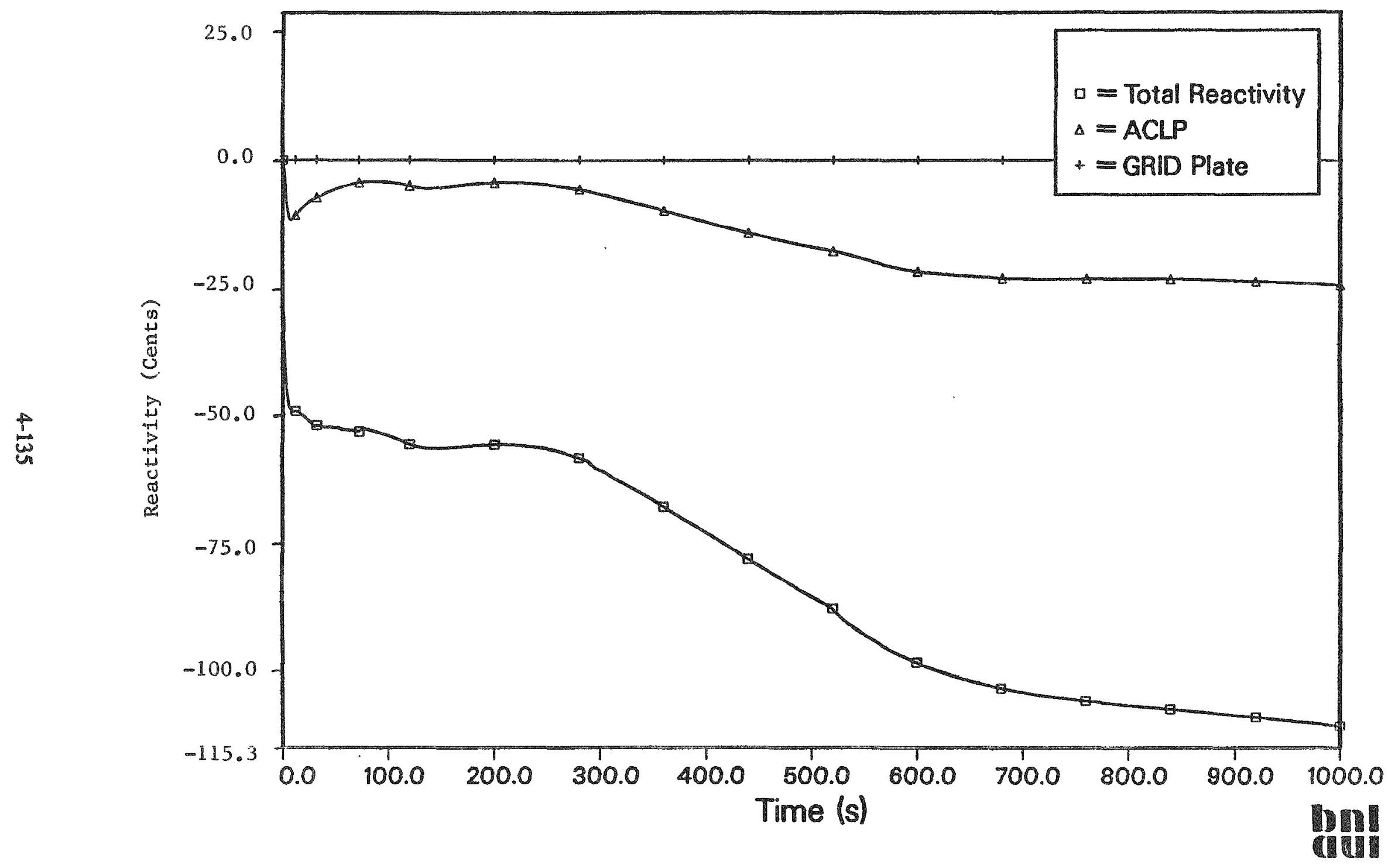

Figure 88. Predicted Total, ACLP, and Grid Plate Reactivity Feedback from SSC or a ULOF with 1 Pump seized. 


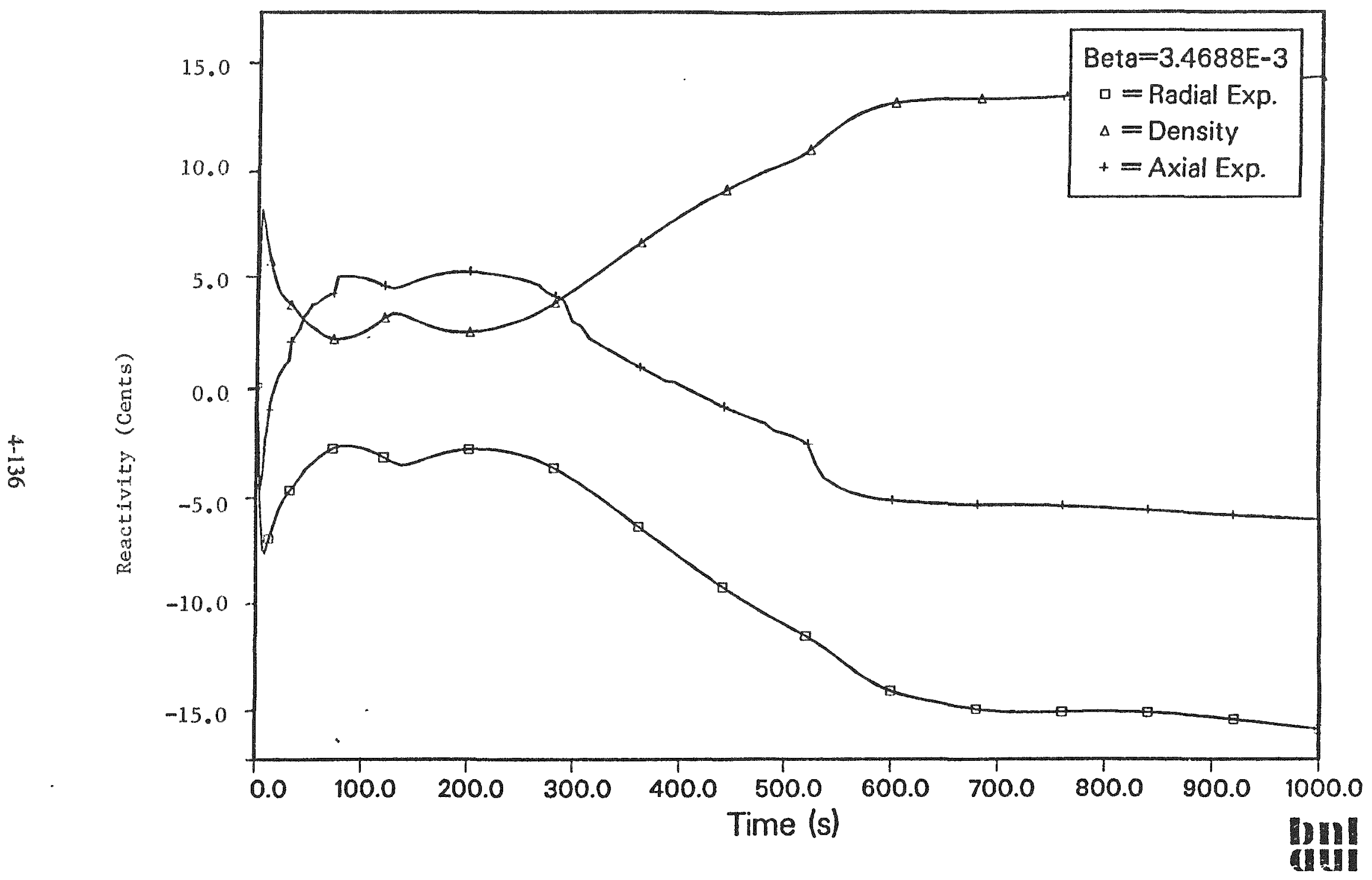

Figure 89. Predicted Core Radial Expansion, Sodium Density, and Axial Expansion Reactivity Feedback from SSC for a ULOF with 1 Pump Seized. 


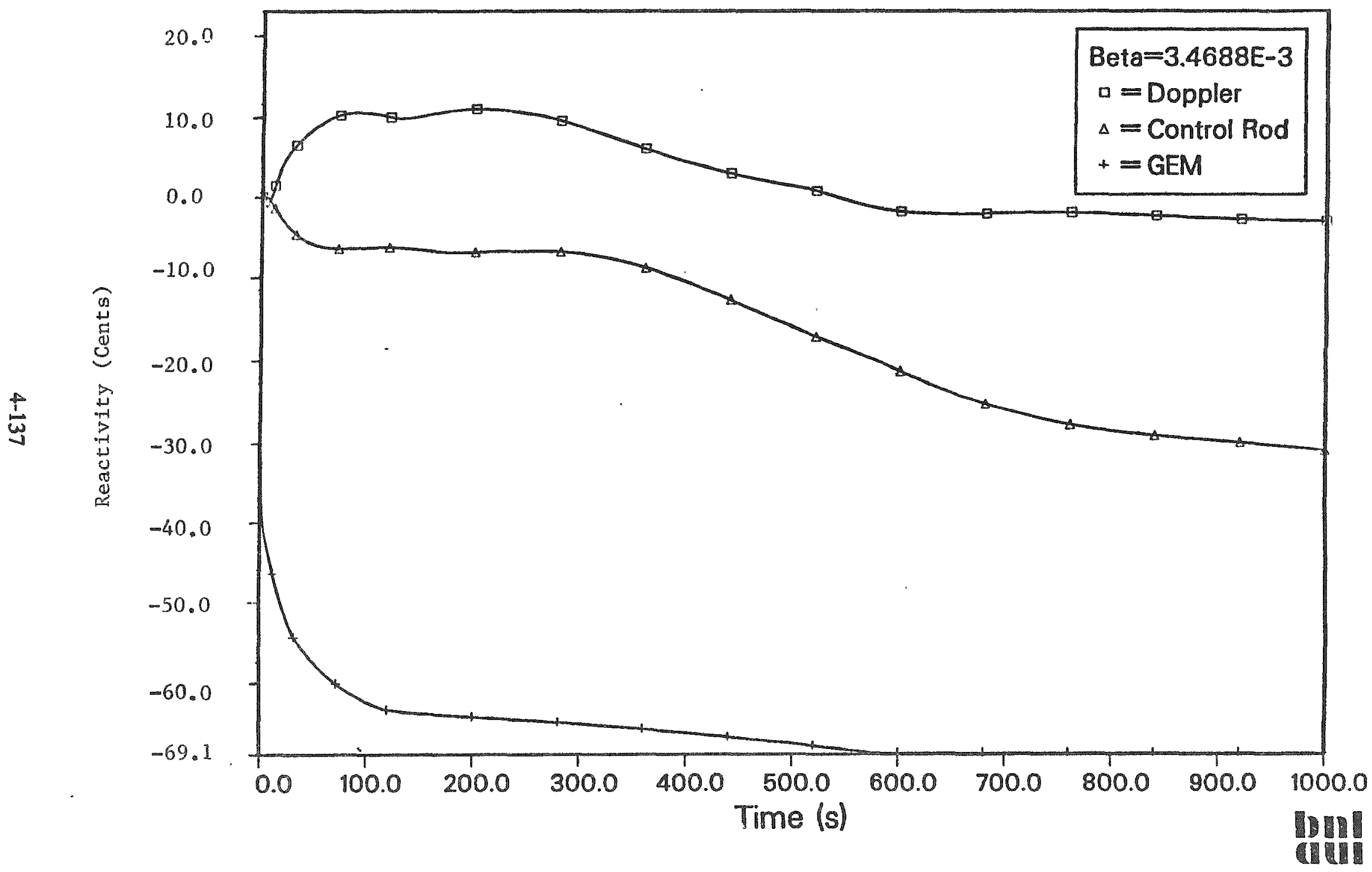

Figure 90. Predicted Doppler, Control Rod Drive Line Thermal Expansion and GEM Reactivity Feedback from SSC for a ULOF with 1 Seized Pump. 
first several seconds. This is triggered by the rapid drop in the sodium level within the GEMs, as shown in Figure 91. which drops quickly as the pumping is cut back sharply.

Peak temperatures calculated for this event are shown in Figures 92 through 95 . The peak fuel temperatures in the hot driver near the axial center of the core, shown in Figure 92, are at the beginning of the event. This is because the negative reactivity from the GEMs drops the power quickly and brings the fuel temperatures down with it. The sodium temperatures near or at the outlet of the core, are shown in Figures 93 through 95. The sodium temperatures peak at the initiation of the event, but reduce with the flow rate.

The only real safety concern here would be the cladding temperatures, and this depends on what assumptions are made regarding the cladding damage limits (see Section 4.16.3.2). This event would have to continue for a long time for significant cladding damage to occur, and even then it would be localized to the hotter parts of the core. Therefore, this event now looks fairly mild, with the GEMs making a significant contribution to reducing its severity.

While we did not analyze the highly improbable case where two coastdowns are missing, it is possible to extrapolate from other cases. We would expect some localized fuel melting and significant cladding damage, but sodium boiling would be very unlikely.

\subsection{Instantaneous Stoppage of One Pump Without Scram}

In principle, a pump could fail in such a way that no coastdown would occur, although it is questionable whether this would develop with warning signs. However, given the reduced role of the operator in ruming the PRISM reactors it's possible that any warning signs would be overlooked. If we also impose a failure to scram the reactor, the probabilities become pretty low. However, one could envision an external event, particularly a large earthquake causing multiple failures and conceivably starting this type of event.

This type of event was analyzed for the previous version of PRISM, using the MINET Code. Because of the short-circuiting of the flow, the reduction of sodium flow to the core was almost $50 \%$. Even so, our SSC calculations indicated the passive reactor shutdown could bring the power down quickly enough to prevent fuel damage.

For the recent analysis we are able to use the detailed pump models. Also, we were able to factor in the newer system pressure drops for the increased core height (from the previous design). Because the head curves are very steep in comparison to those we assumed in the previous analysis, there was less surging in the three pumps remaining operational. Therefore, the coolant flow through the core dropped quickly to $49 \%$ of the rated flow.

The normalized sodium flow rate through the reactor, as specified for SSC, and the normalized power production, as calculated by SSC, are shown in Figure 96 . The reasons for the rapid decrease in power can be seen in Figures 97 through 99, which include all of the important reactivity feedbacks. Note that the feedback from the GEMs, roughly -25 cents, develops quickly and dominates the other feedbacks. This is because the sudden loss of a pump drops the pressure quickly, dropping the sodium level in the GEMS.

Key reactor and system temperatures are shown in Figures 100 through 103 . With reduced pumping and less power production, system temperatures fall significantly. However, since the power does not remain as low as the coolant flow, the temperature rise across the core and the temperature fall 


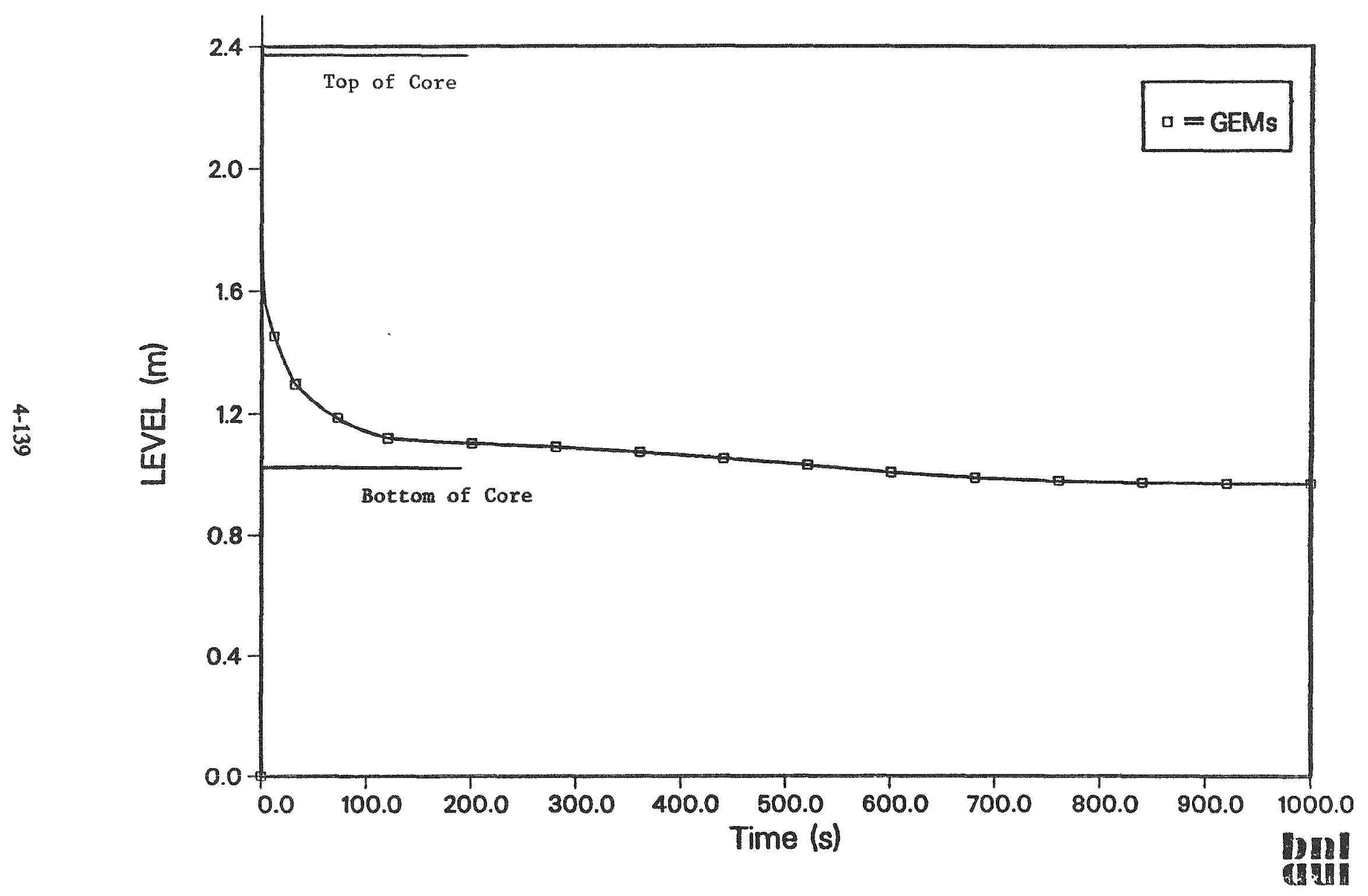

Figure 91. Predicted Sodium Level in GEMs from SSC for a ULOF with 1 Pump Seized. 


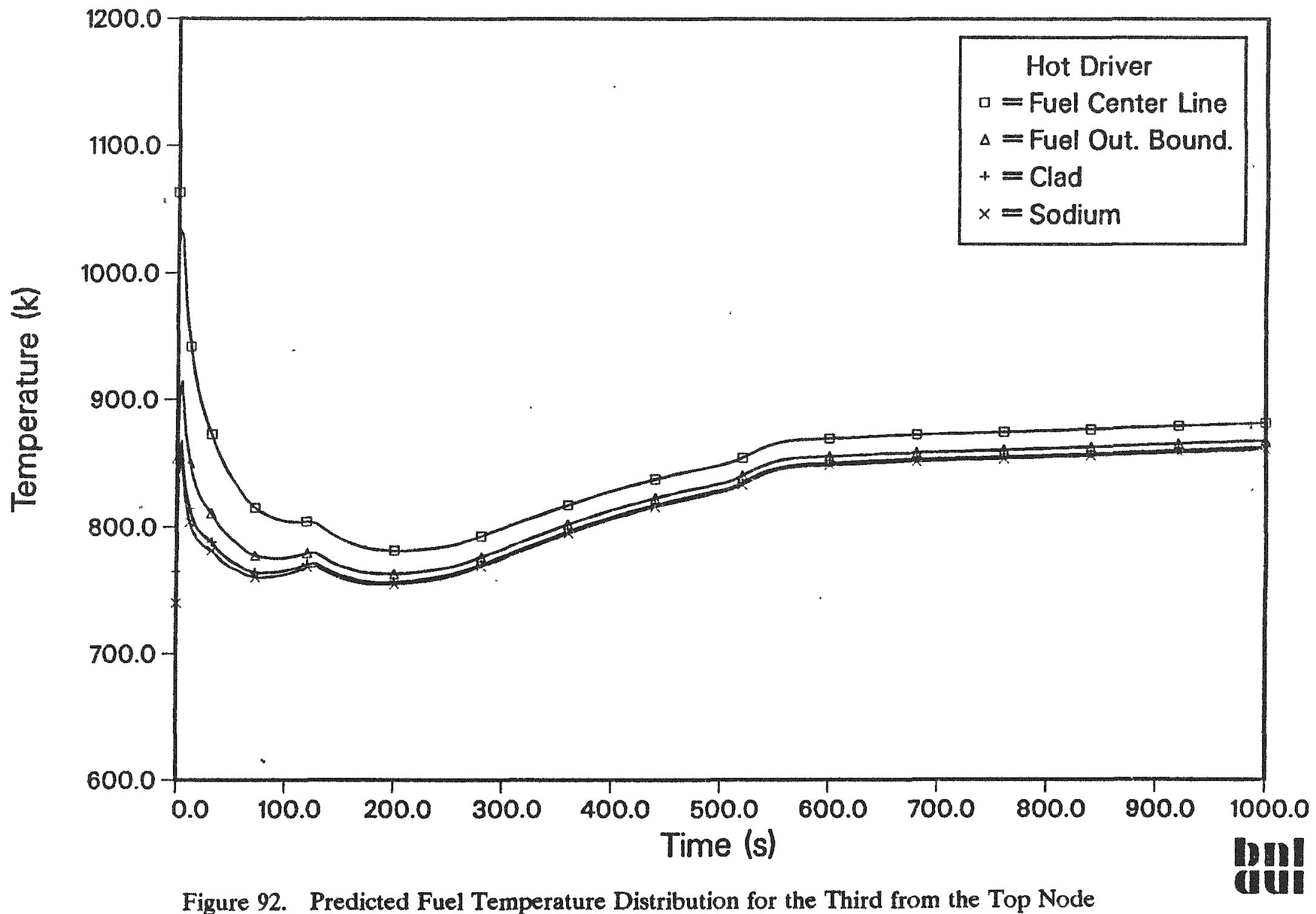

Figure 92. Predicted Fuel Temperature Distribution for the Third from the Top Node (i.e., $0.897 \mathrm{~m}-0.673 \mathrm{~m}$ ) of a Fuel Pin from SSC for a ULOF with 1 Pump Seized. 


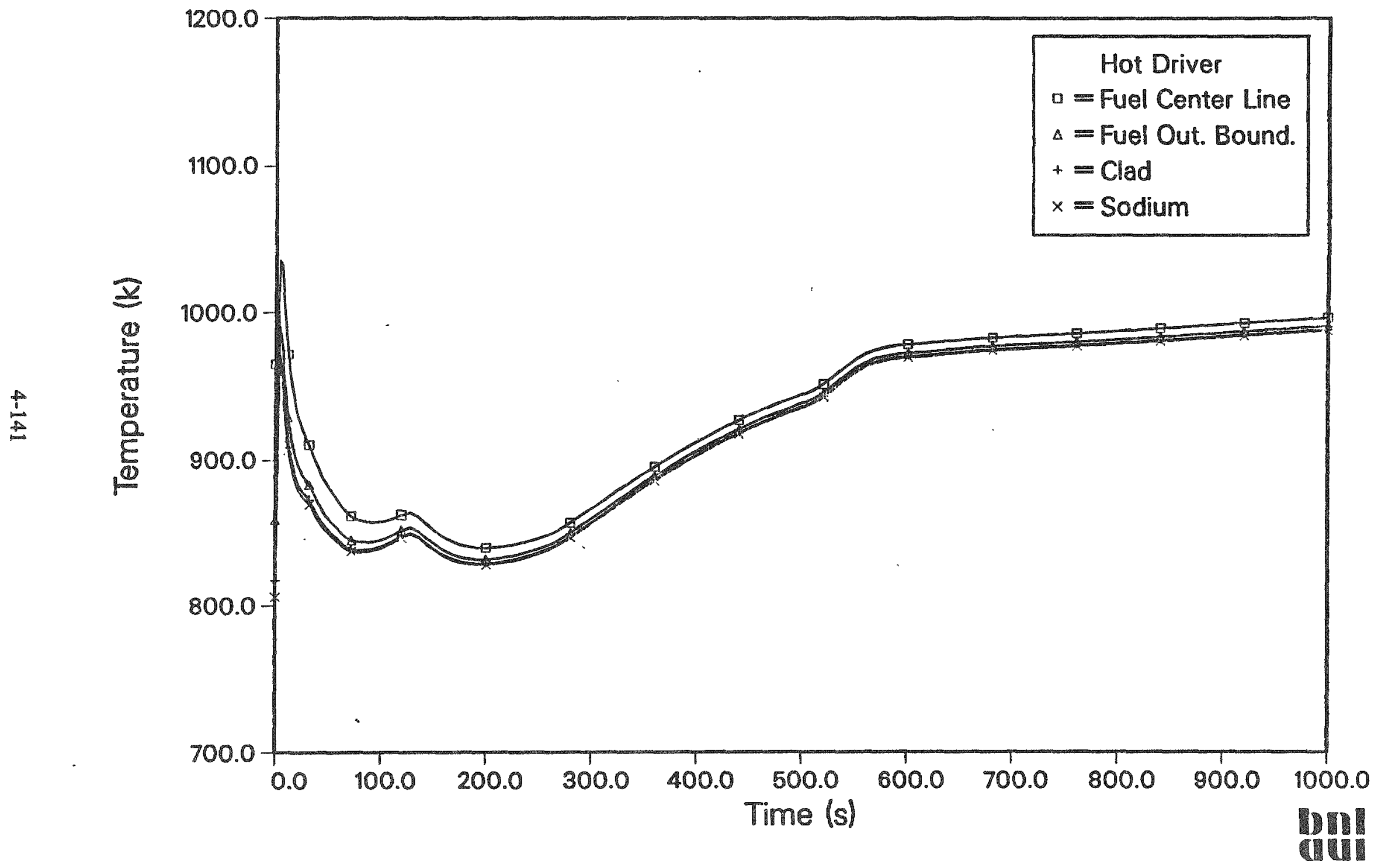

Figure 93. Predicted Fuel Temperature Distribution for the Top Node (i.e., $1.346 \mathrm{~m}$ $1.122 \mathrm{~m}$ ) of a Fuel Pin from SSC for a ULOF with 1 Seized Pump. 


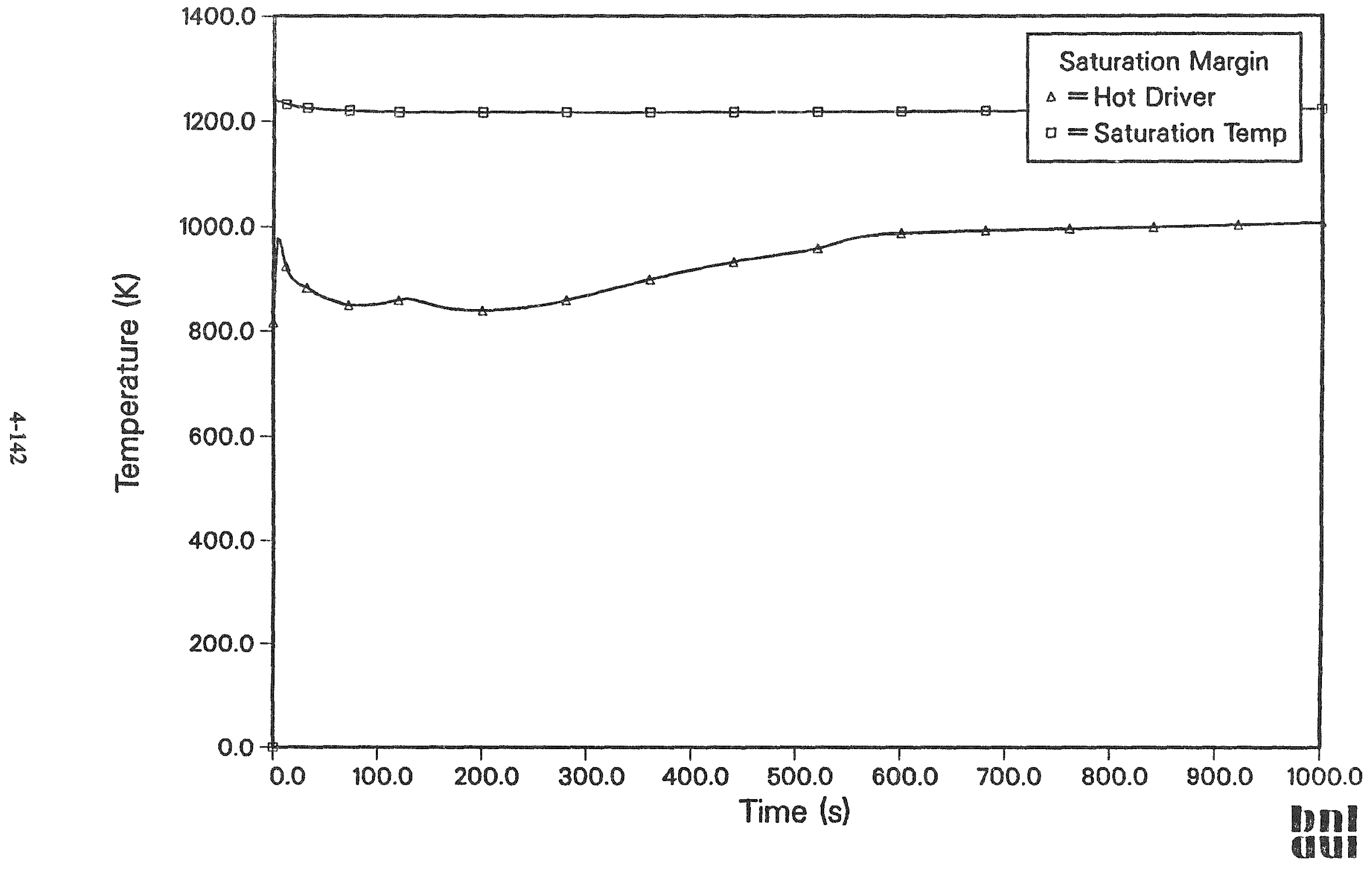

Figure 94. Predicted Margin to Sodium Saturation from SSC for a ULOF with 1 Pump Seized. 


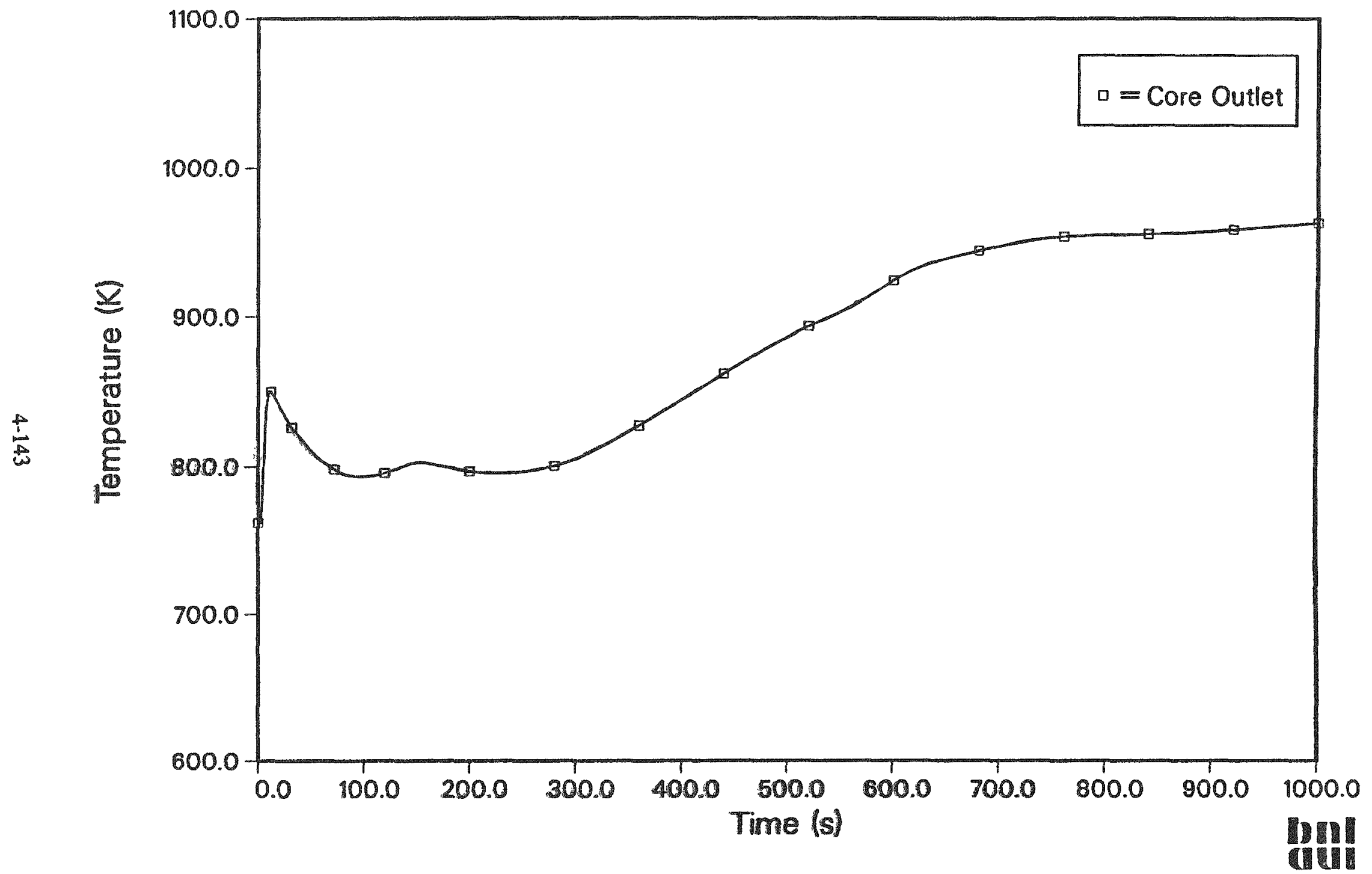

Figure 95. Core Exit Average Sodium Temperature from SSC for a ULOF with 1 Pump Seized. 


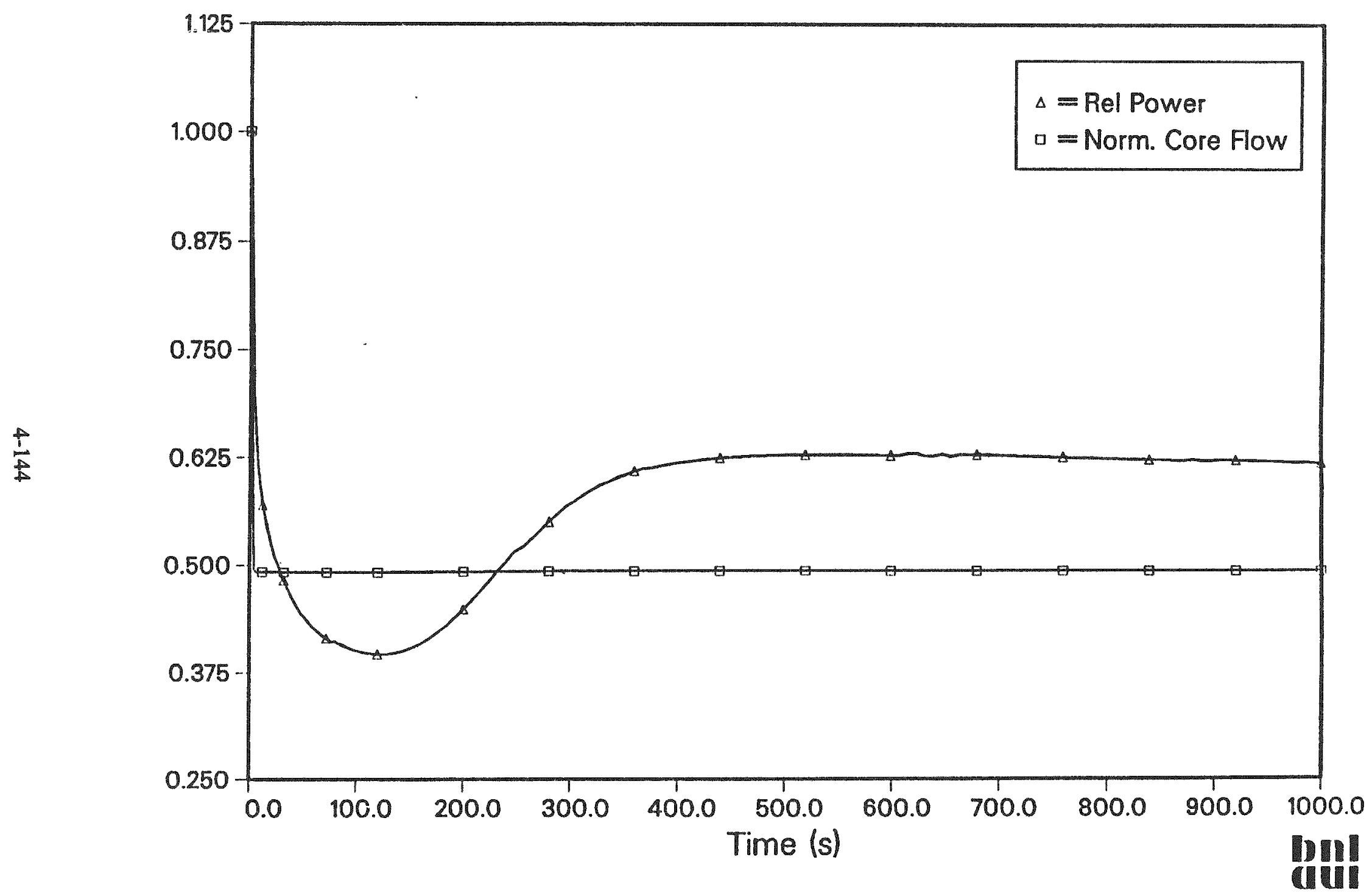

Figure 96. Predicted Relative Power and Normalized Core Flow from SSC for a Pump Seizure. 


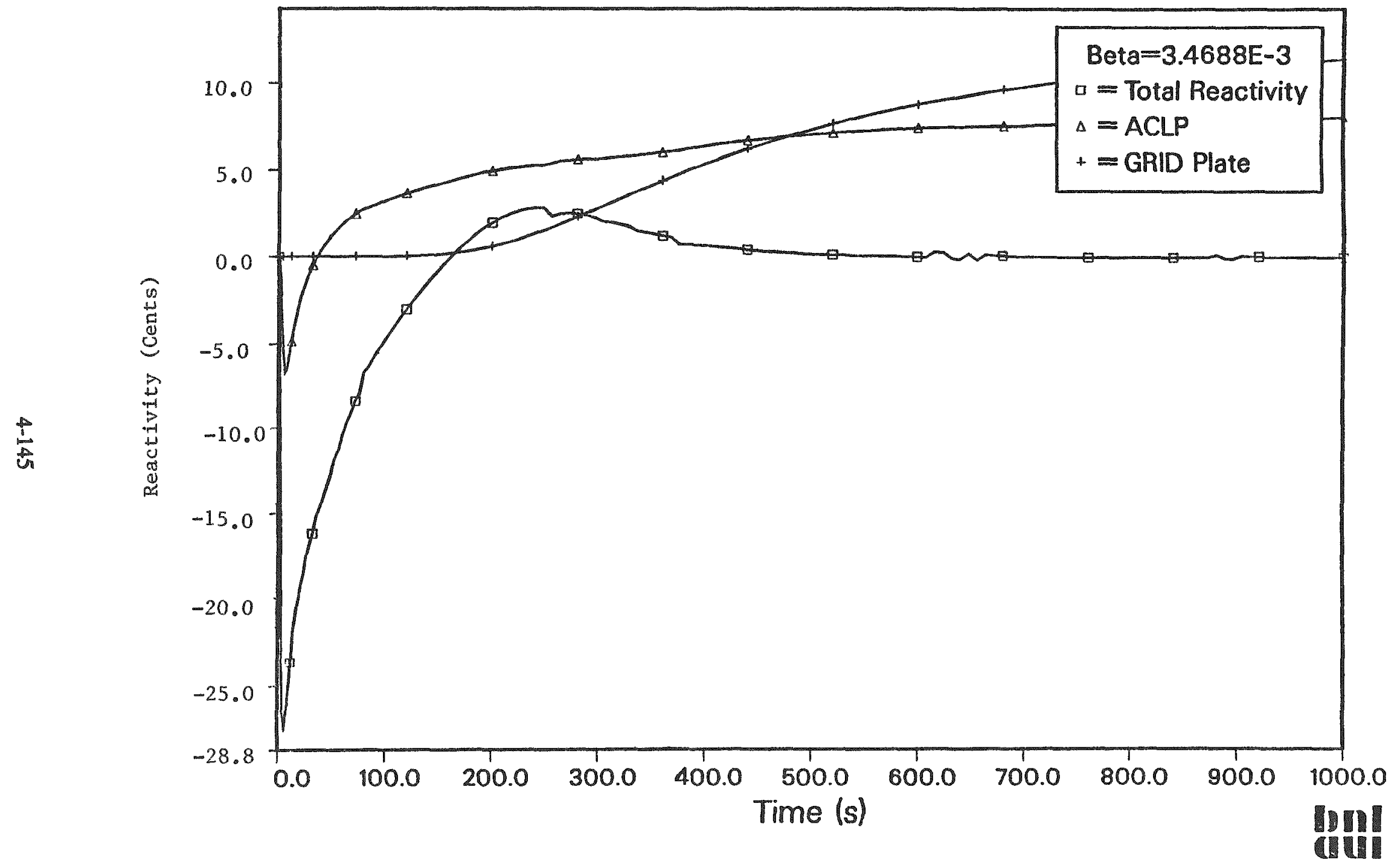

Figure 97. Predicted Total, ACLP, and Core Support GRID Plate Reactivity Feedback from SSC for a Pump Seizure. 


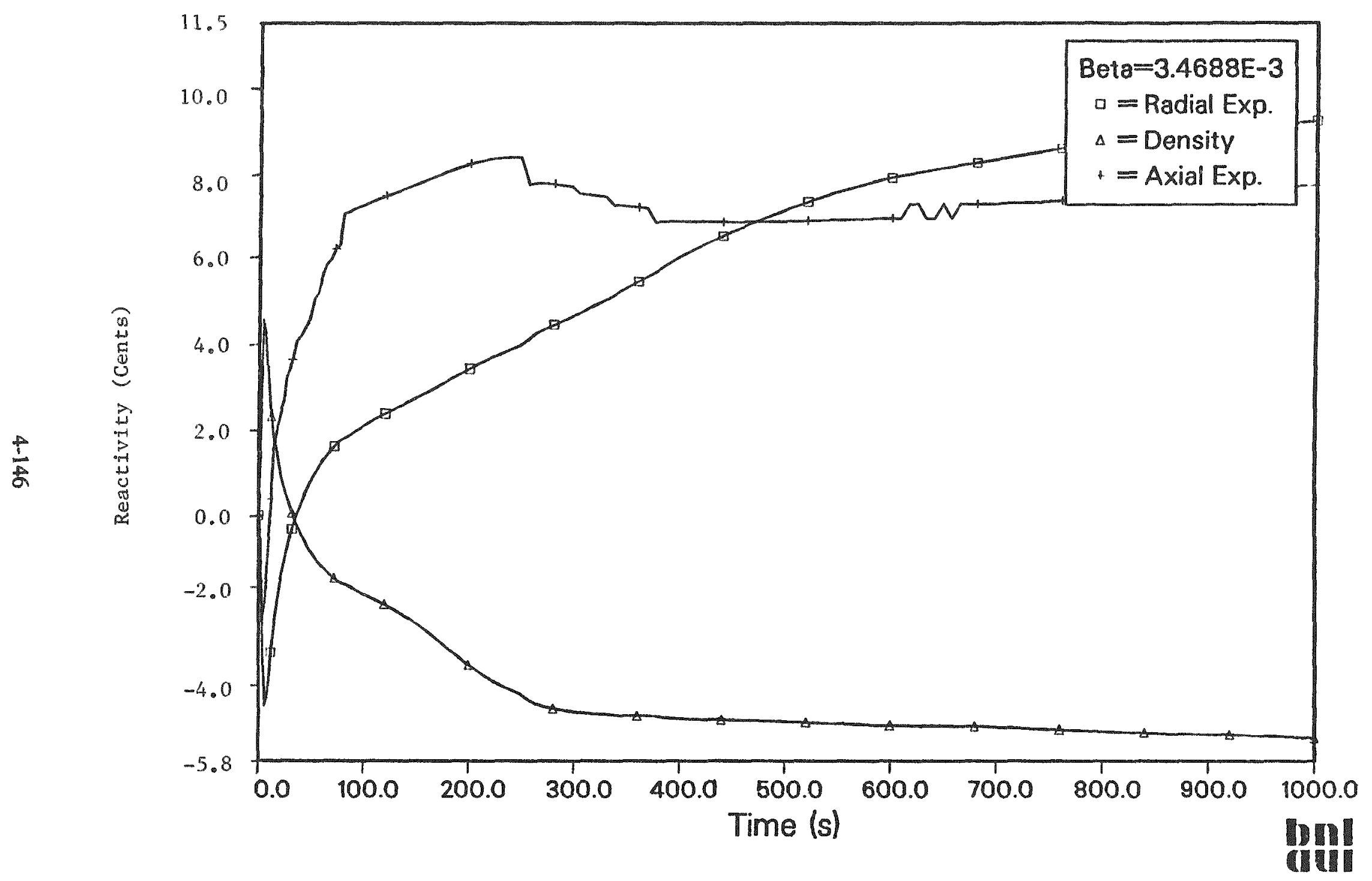

Figure 98. Predicted Core Radial Expansion, Sodium Density, and Axial Expansion Reactivity Feedback from SSC for a Pump Seizure. 


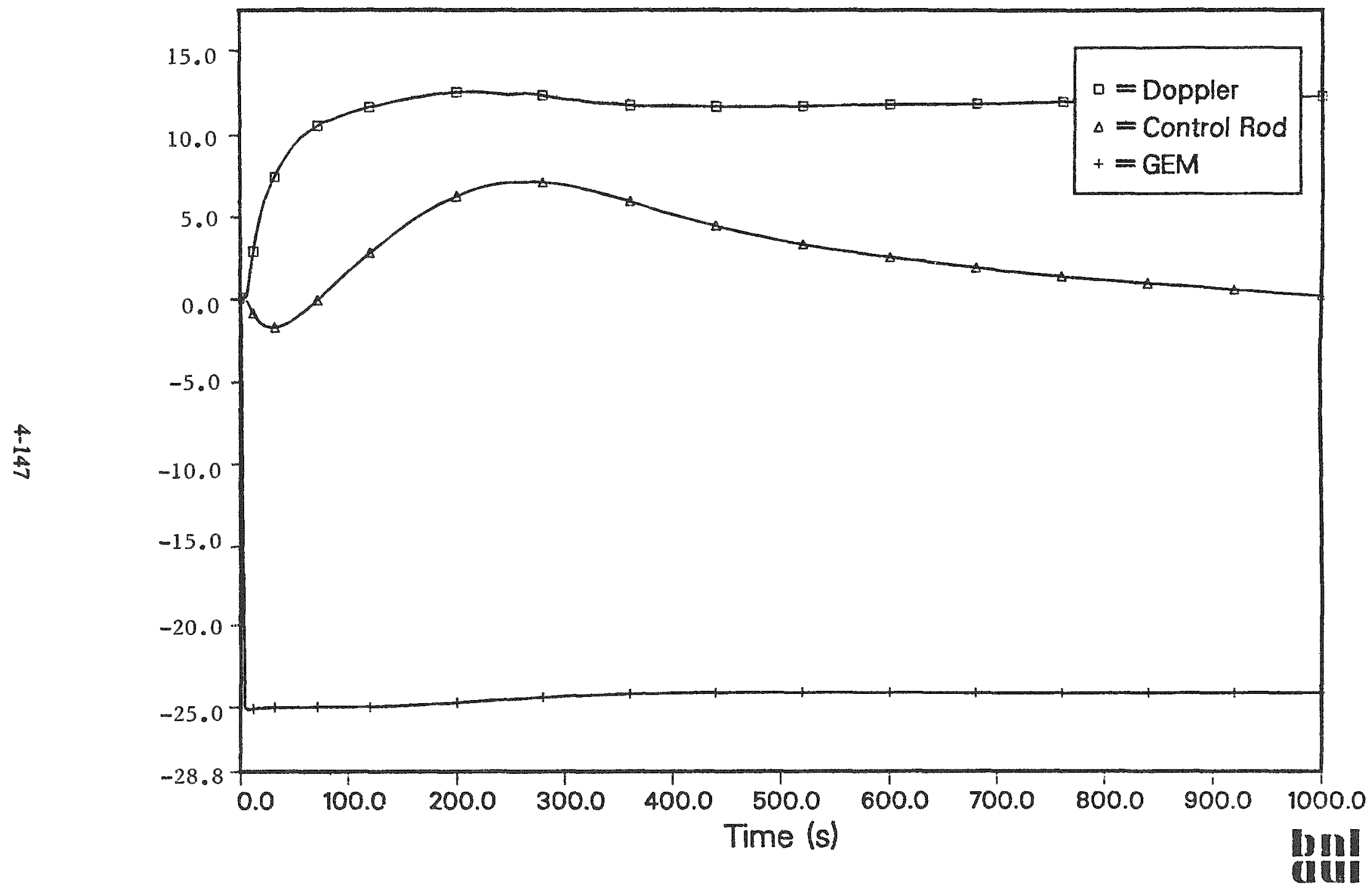

Figure 99. Predicted Doppler, Control Rod Drive Thermal Expansion, and GEM Reactivity Feedback from SSC for a Pump Seizure. 


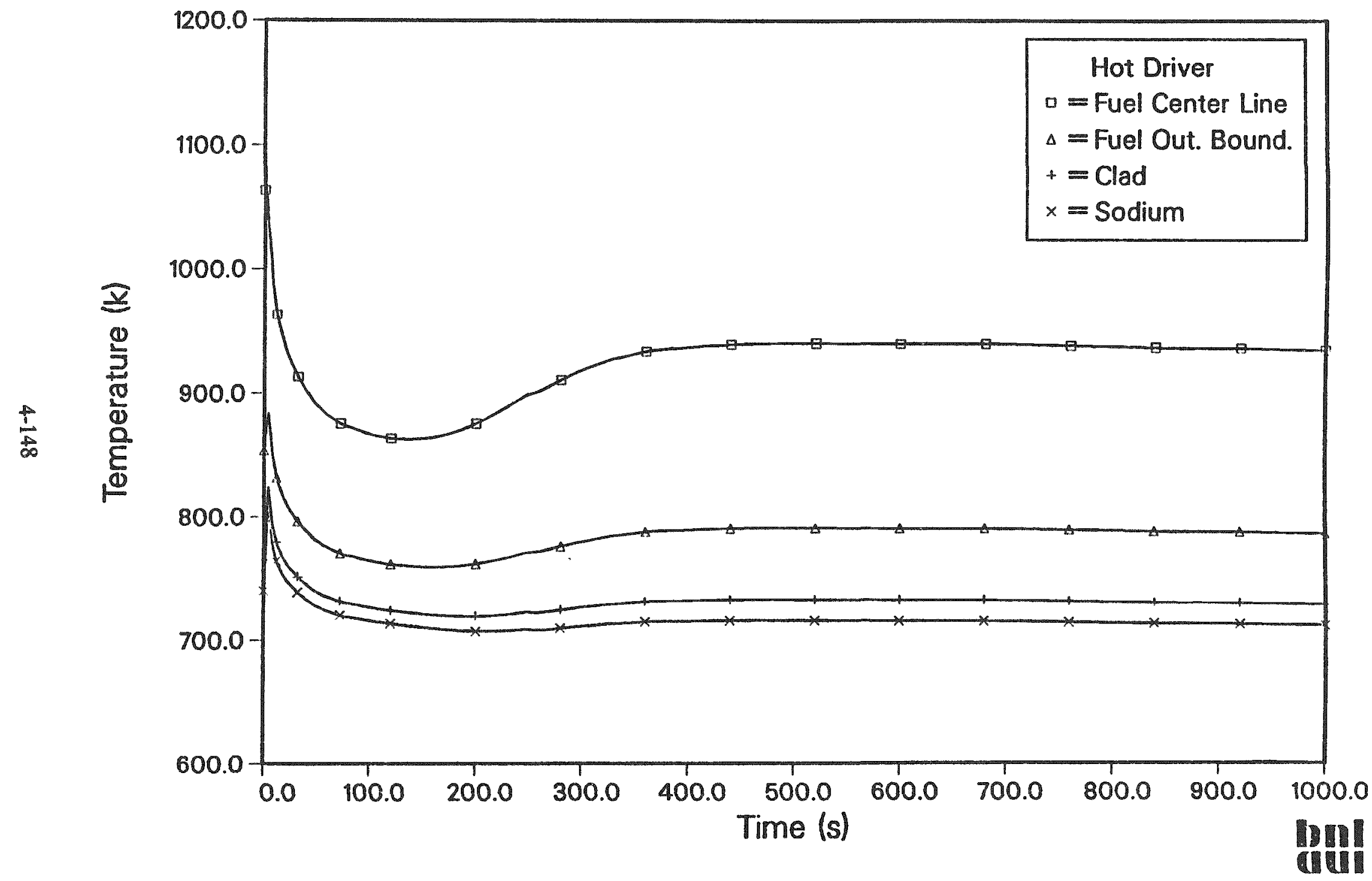

Figure 100. Predicted Fuel Temperature Distribution from SSC for the Third Node from the Top (i.e., $0.897 \mathrm{~m}-0.673 \mathrm{~m}$ ) for a Pump Seizure. 


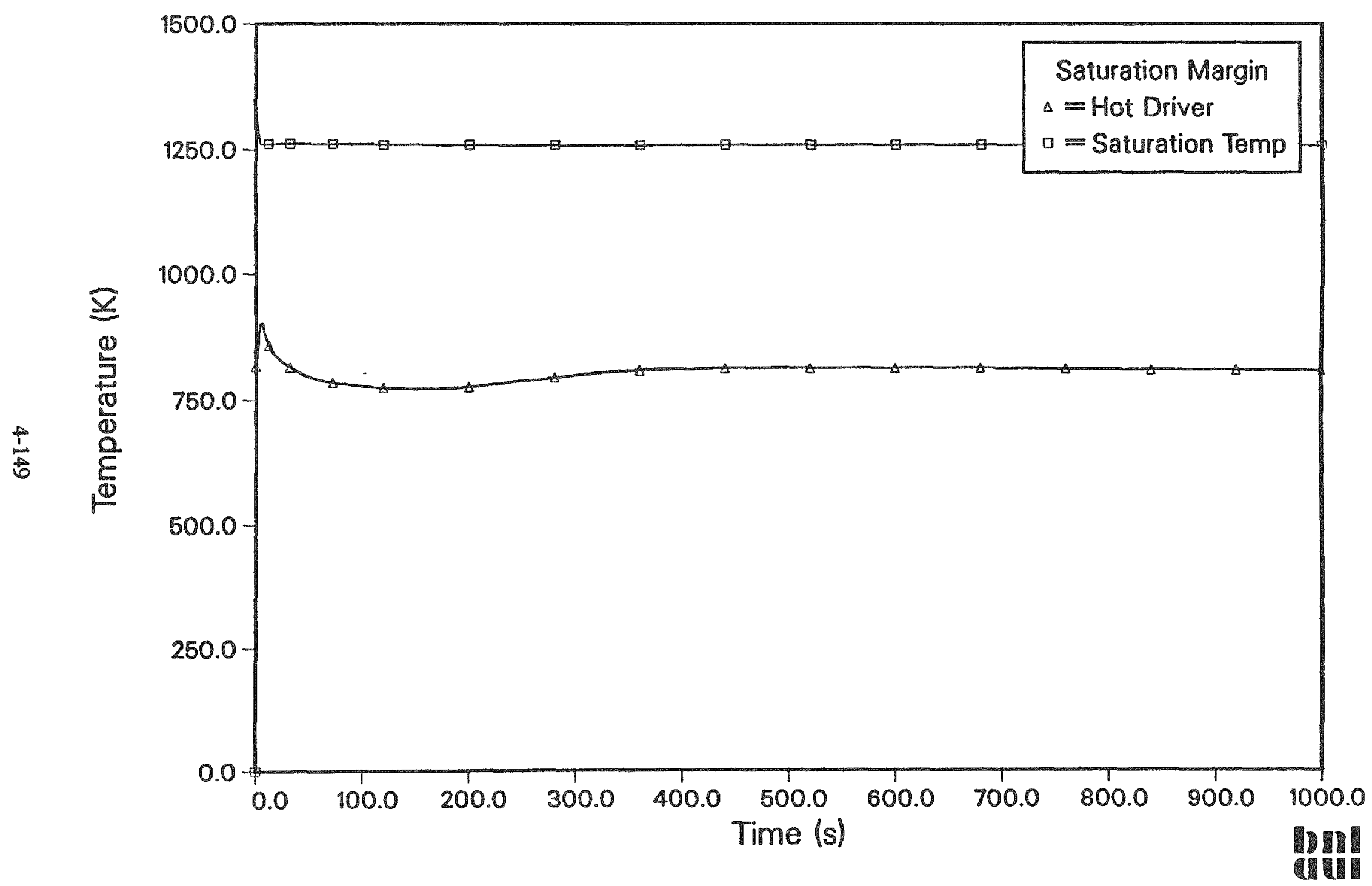

Figure 101. Predicted Margin to Sodium Saturation from SSC for a Pump Seizure. 


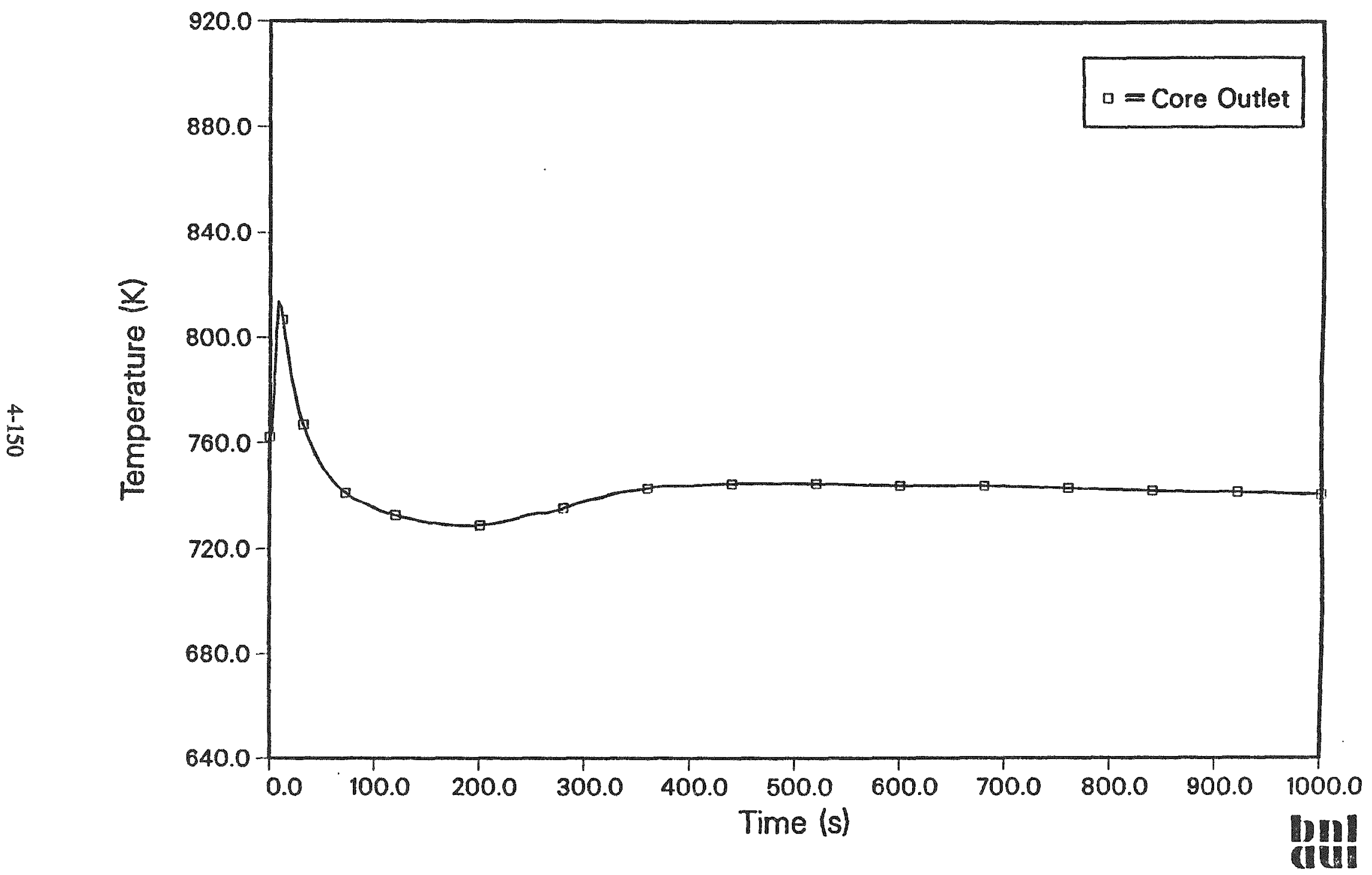

Figure 102. Core Outlet Average Sodium Temperature from SSC for a Pump Seizure. 


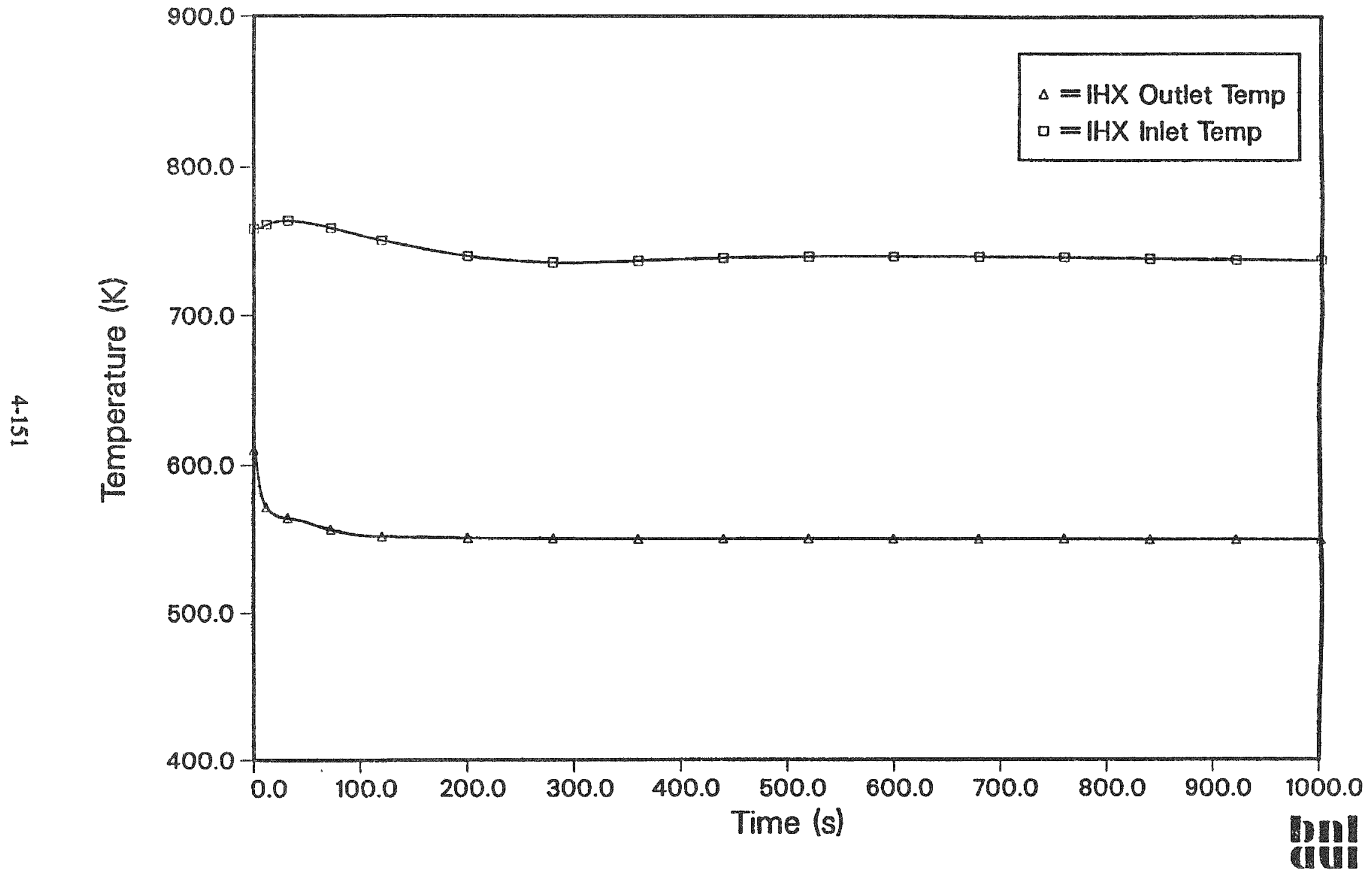

Figure 103. Predicted IHX Inlet and Outlet Sodium Temperatures from SSC for a Pump Seizure. 
across the IHX increase, as indicated in Figure 103. Also, as shown in Figure 100, the drop in temperatures are quite sudden, so the peak maximum temperatures are reached within the first 5 seconds.

Thus, our analyses, using both the MINET and SSC codes, indicates this event is largely benign. Clearly the GEMs can have a major impact when one pump is stopped suddenly.

\subsection{Instantaneous Stoppage of All Pumps Without Scram}

This event goes well beyond bounding events in probability space and could best be described as "exceedingly unlikely". In principle, a massive earthquake might be postulated that causes the loss of all energy flow (including that from the synchronous machines) to the EM pumps and completely incapacitates the scram system. If, in addition, all this occurs simultaneously and the operator doesn't trigger the USS, then this exceedingly unlikely event could occur.

Our reason for analyzing this event was to answer an intriguing "what if?" question, particularly in light of the recent addition of the GEMs. We believed there was some chance that the GEMs might bring the power down quickly enough to prevent sodium boiling. However, our analysis currently shows that for this event the sodium would boil after about 10 seconds, and that a power excursion would likely result.

The first task in the analysis was to determine the rate of flow reduction to the reactor. We used the same MINET representation as was discussed previously except this time the pump heads were cut to zero within four seconds. The calculated reactor flow rate is shown in Figure 104. The flow rate curve labelled "General Electric, ARIES" is from a very preliminary calculation, and should not be regarded as final. However, it is interesting that the flow rate estimated by GE decreases much more slowly than that from our MINET calculation. The inertia that is apparently in the GE calculation is not fully understood. We believe the sudden flow reduction predicted by MINET is more realistic. However, if the curve predicted by the applicant is correct, that would surely make for a slower transient, and sodium boiling would be delayed at the very least.

The SSC calculations were driven, using the pump head, to have the same sodium flow rate shown in Figure 104 (MINET). The resultant reactor power and sodium flow, as calculated by SSC, are shown in Figure 105. It is noted that initially the power decreases, although not nearly as fast as the flow decreases. By 20 seconds, the power is increasing, and a sodium-boiling driven power excursion develops after about 25 seconds. The reactivity feedbacks shown in Figures 106 through 108 tell the full story. The total reactivity (Figure 106) is initially dominated by the feedback from the GEMs (Figure 108), which quickly add $63 \mathrm{c}$ of negative reactivity, but is later dominated by the sodium density/void feedback (Figure 107). The sodium appears to be largely subcooled through the first 14 seconds, but the large scale sodium boiling is developing thereafter. Most of the other feedbacks are much smaller, although the Doppler feedback is accelerating at the end. The one crucial feedback that would have to limit the severity of the event is the axial expansion of the fuel. However, our model is based on thermal expansion and does not include the rapid "prefailure extrusion" (rapid axial fuel expansion) that ANL predicts for rapid temperature increase.

Some other key parameters are shown in Figures 109 through 112 . Not surprising, the sodium levels in the GEMs drop quickly, as shown in Figure 109. Peak temperatures in the hot driver clearly show sodium boiling at 9 seconds, and sodium boiling is developing in the average drivers by 16 seconds, as shown in Figure 112. 


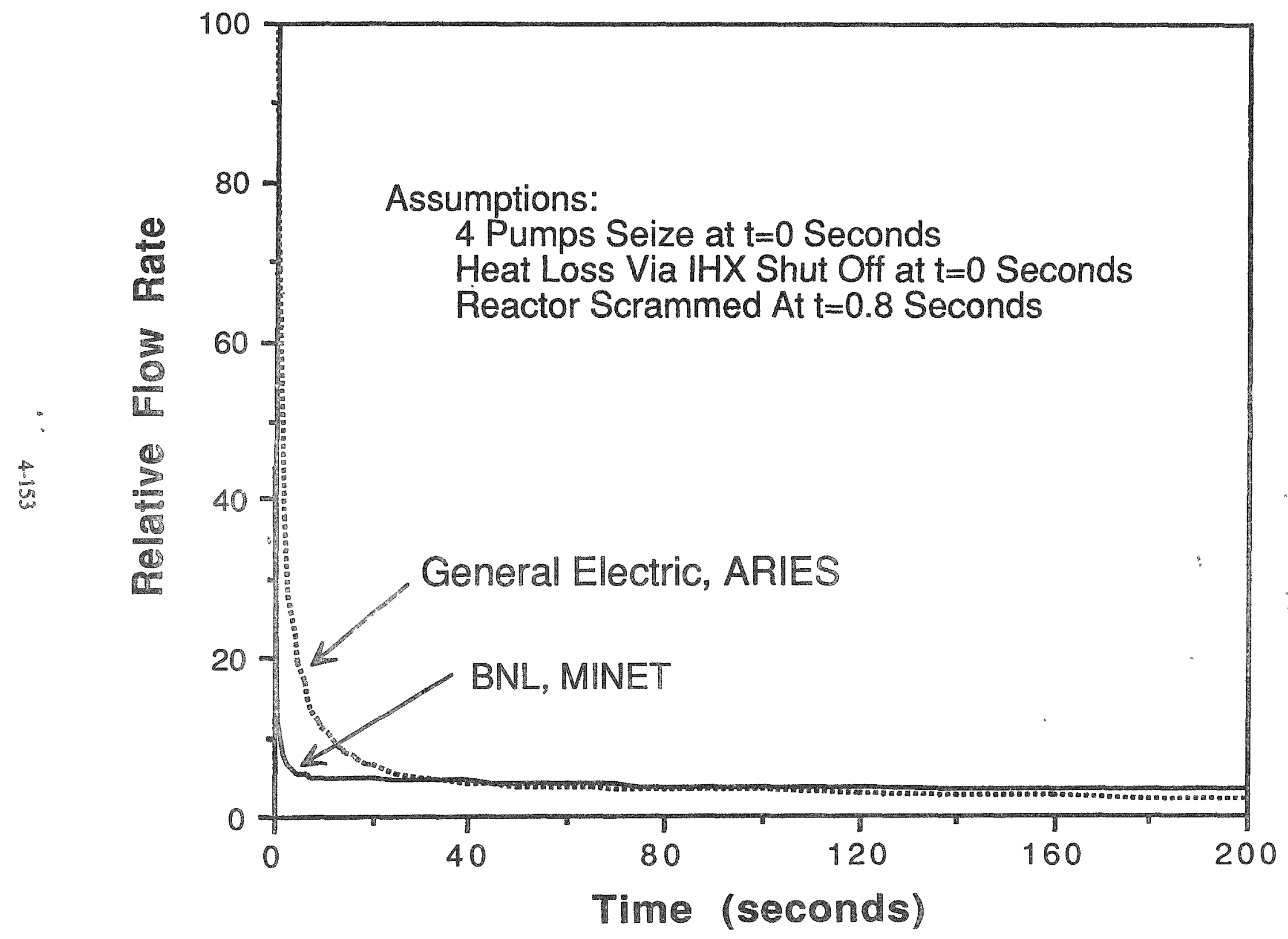

Figur 104. PRISM Core Sodium Flow Rates After 4 Pumps "Seize". 


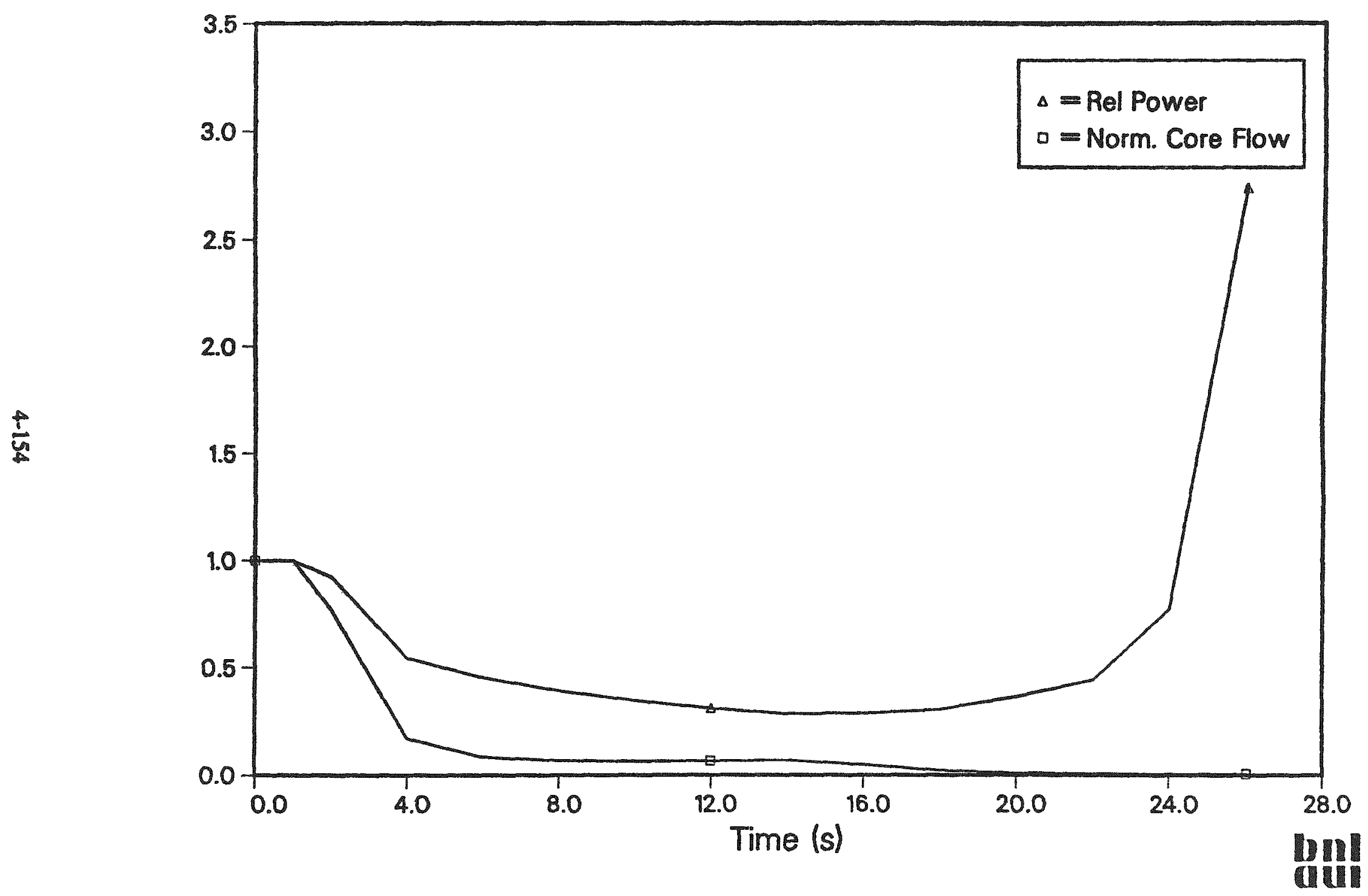

Figure 105. Predicted Relative Power and Normalized Core Flow from SSC for a Pump Seizure. 


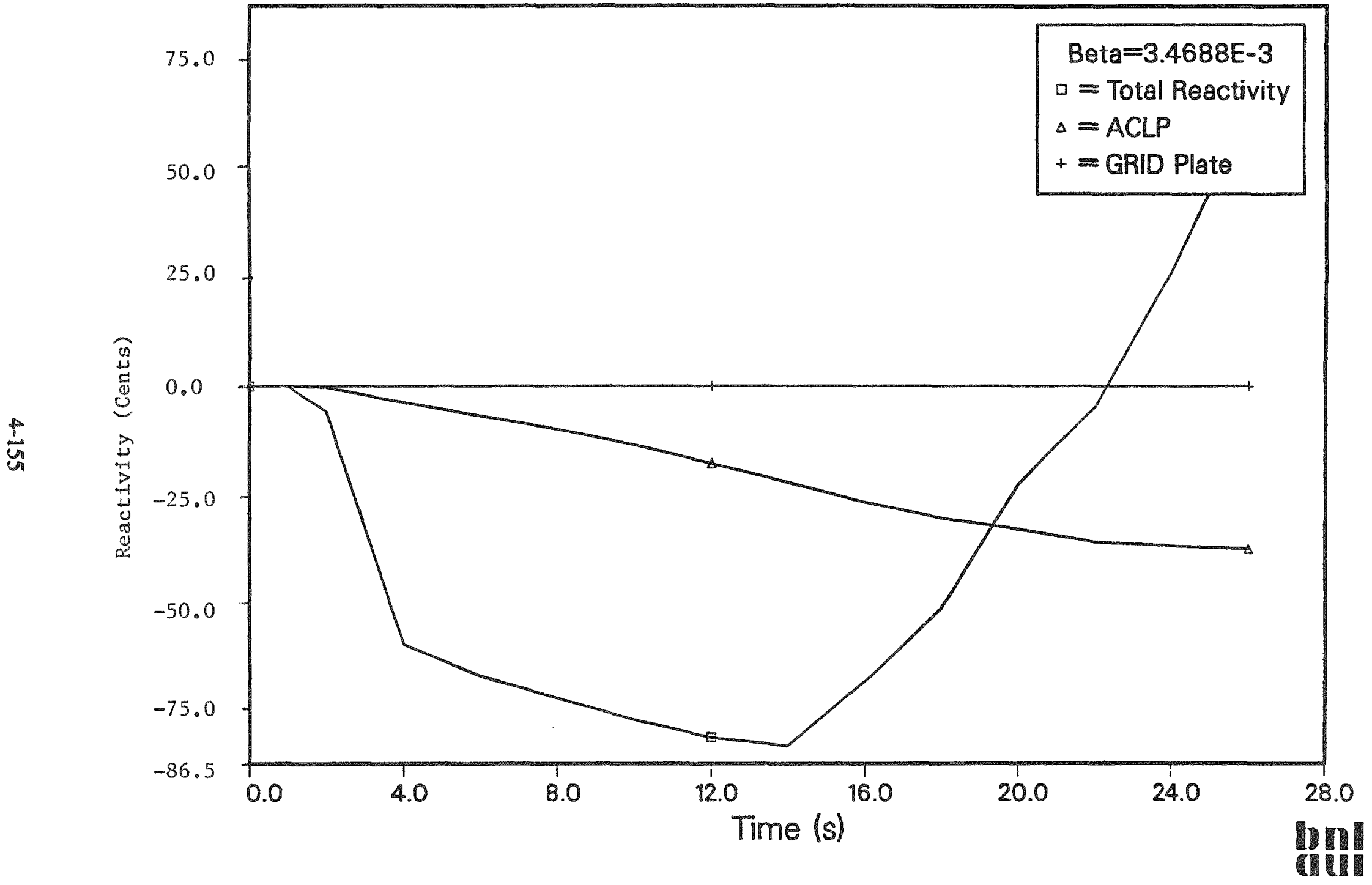

Figure 106. Predicted Total, ACLP, and Core Support GRID Plate Reactivity Feedback from SSC for a Total EM Pump Failure. 


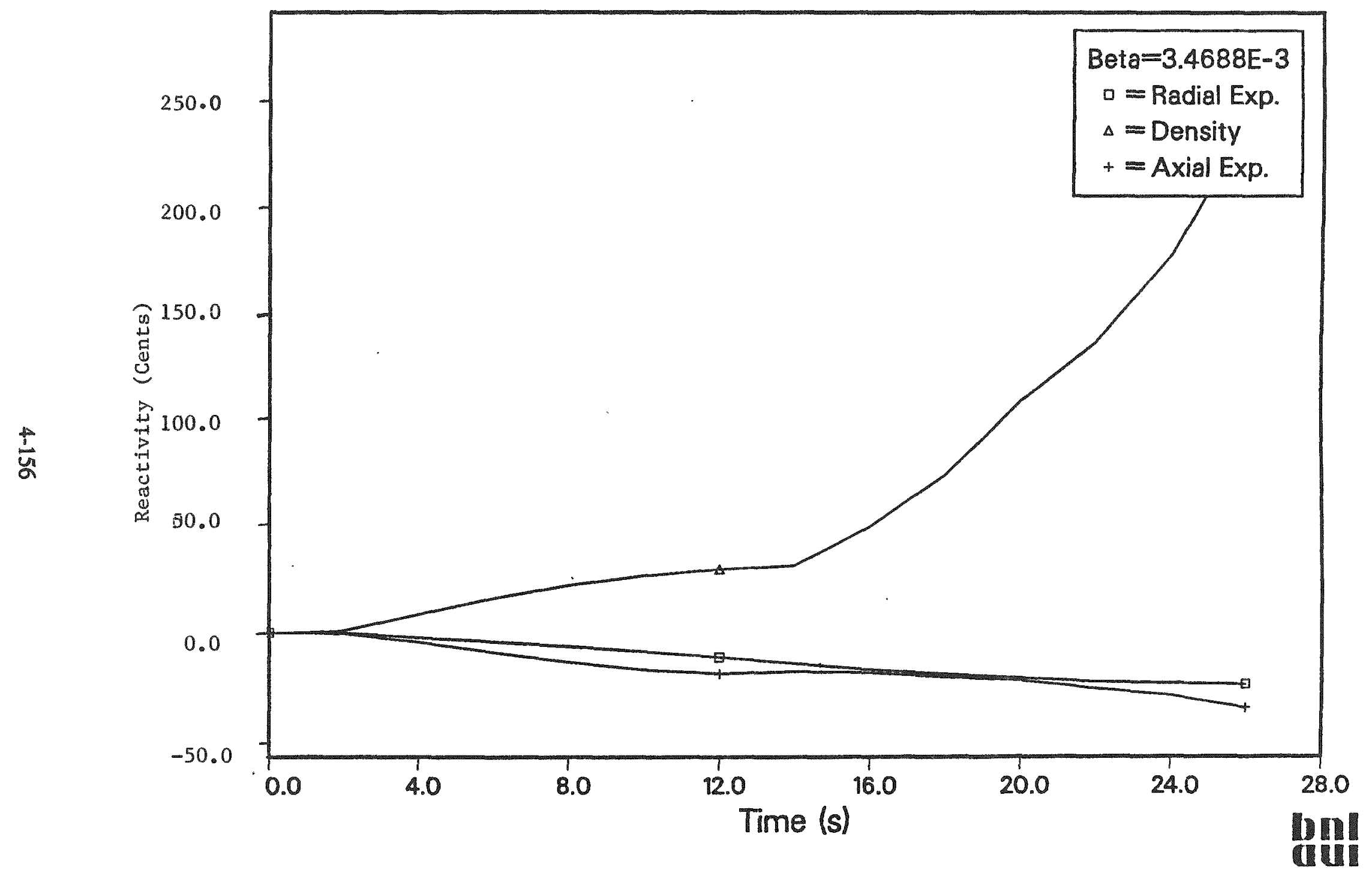

Figure 107. Predicted Core Radial Expansion, Sodium Density, and Axial Expansion Reactivity Feedback from SSC for a Total EM Pump Failure. 


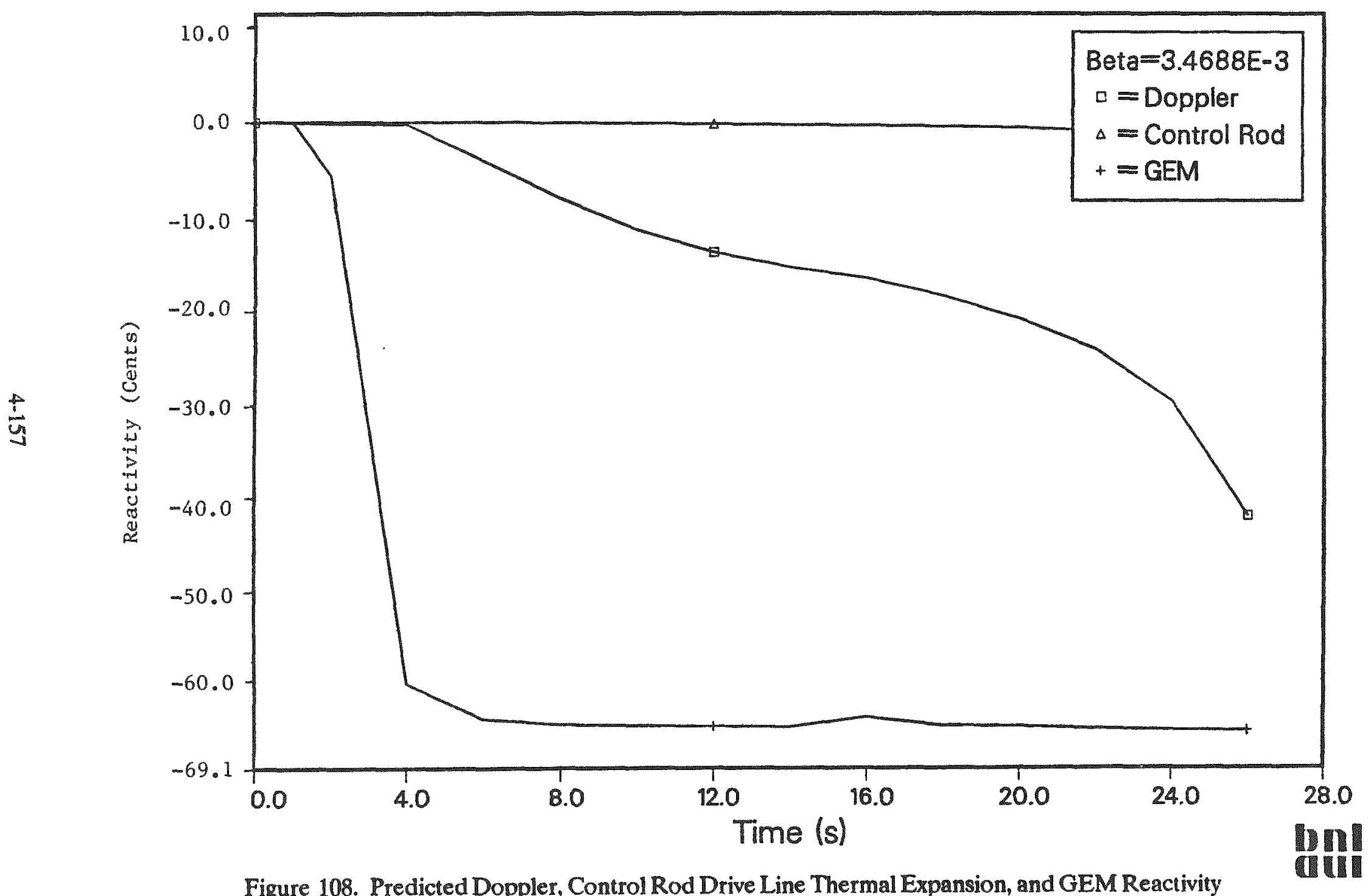

Figure 108. Predicted Doppler, Control Rod Drive Line Thermal Expansion, and GEM Reactivity Feedback from SSC for a Total EM Pump Failure. 


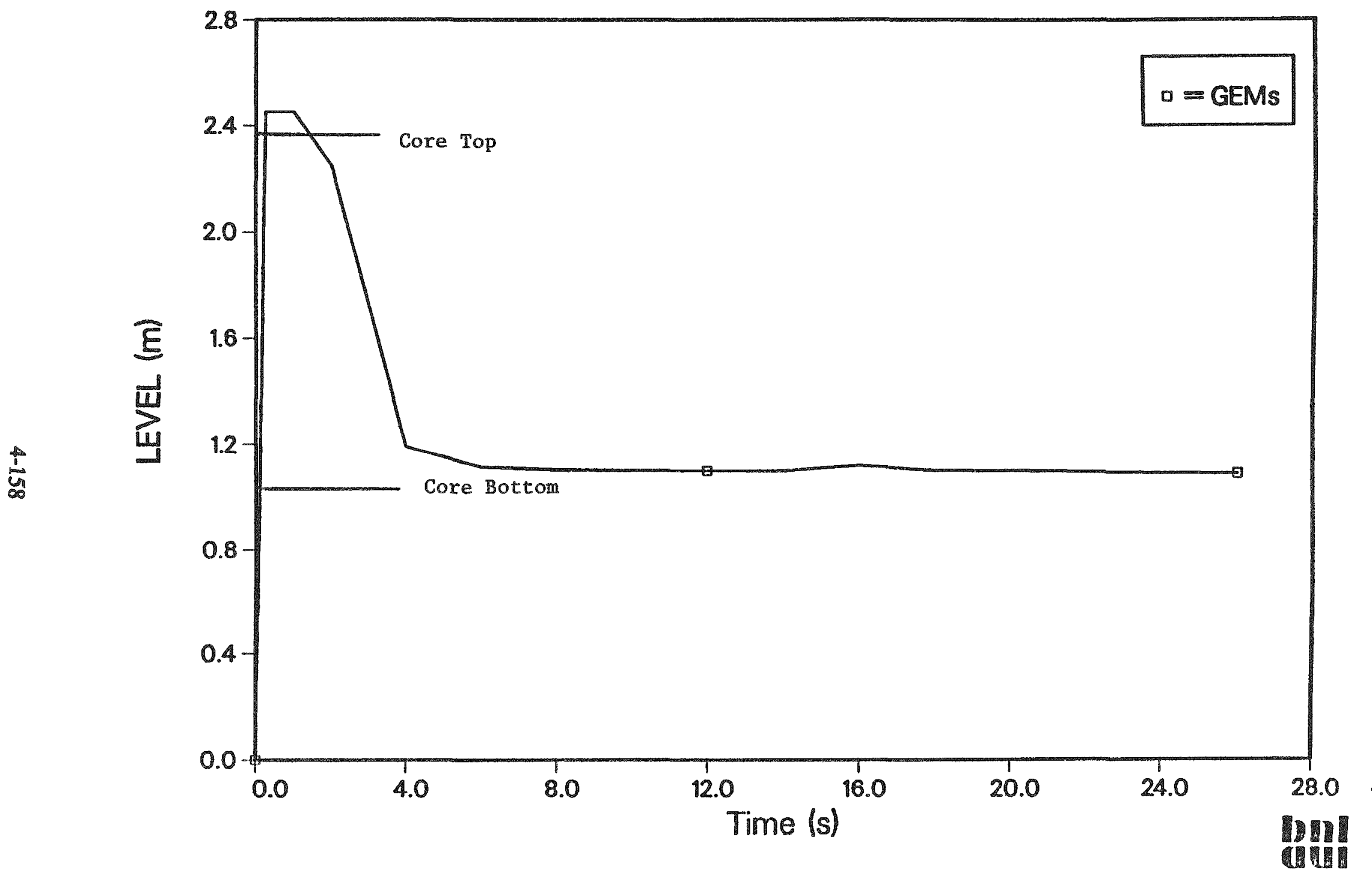

Figure 109. Predicted Sodium Level in the GEMs from SSC for a Total EM Pump Failure. 


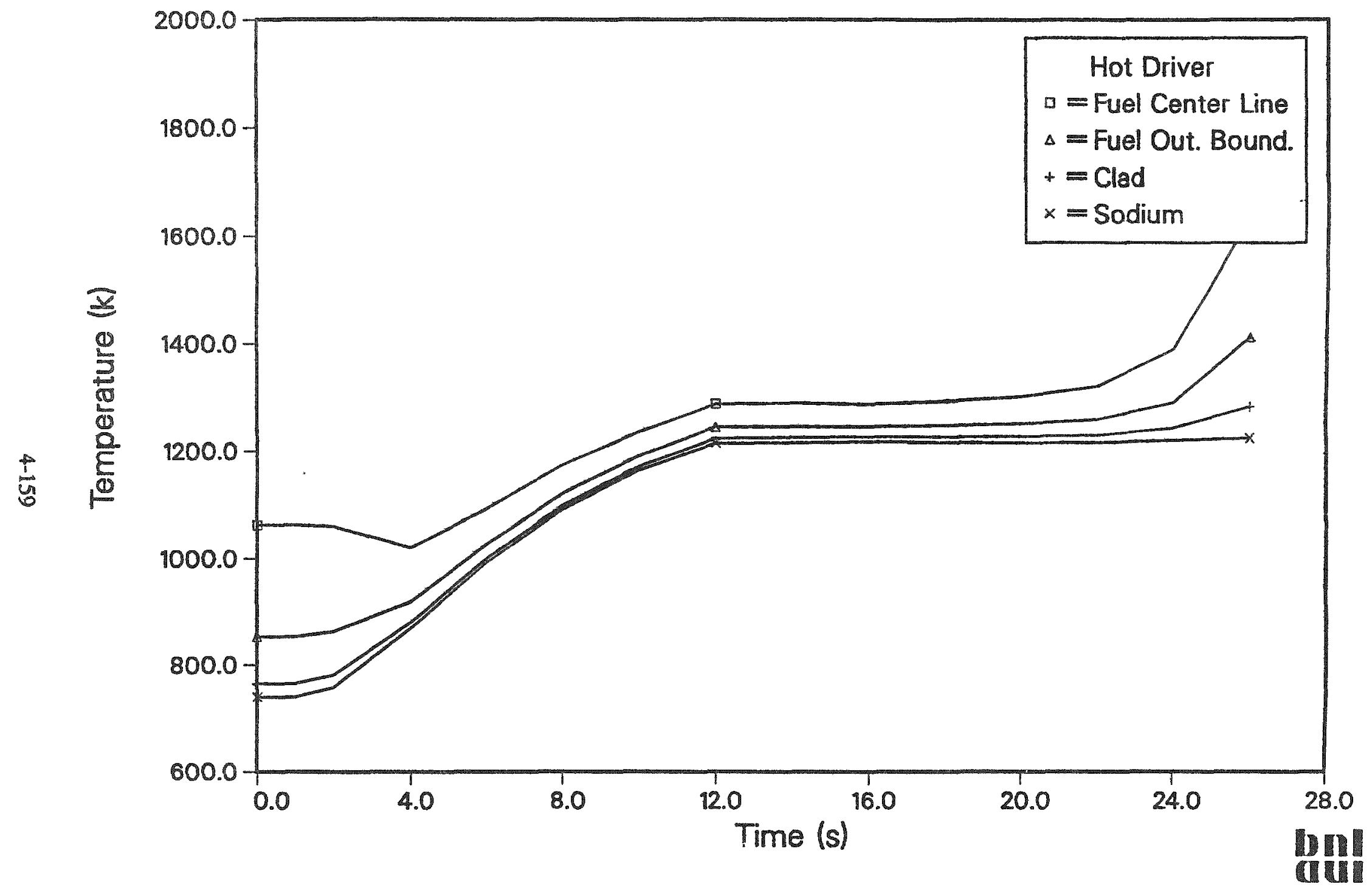

Figure 110. Predicted Fuel Temperature Distribution from SSC for the Third Node from the Top (i.e., $0.897 \mathrm{~m}-0.673 \mathrm{~m}$ ) for a Total EM Pump Failure. 


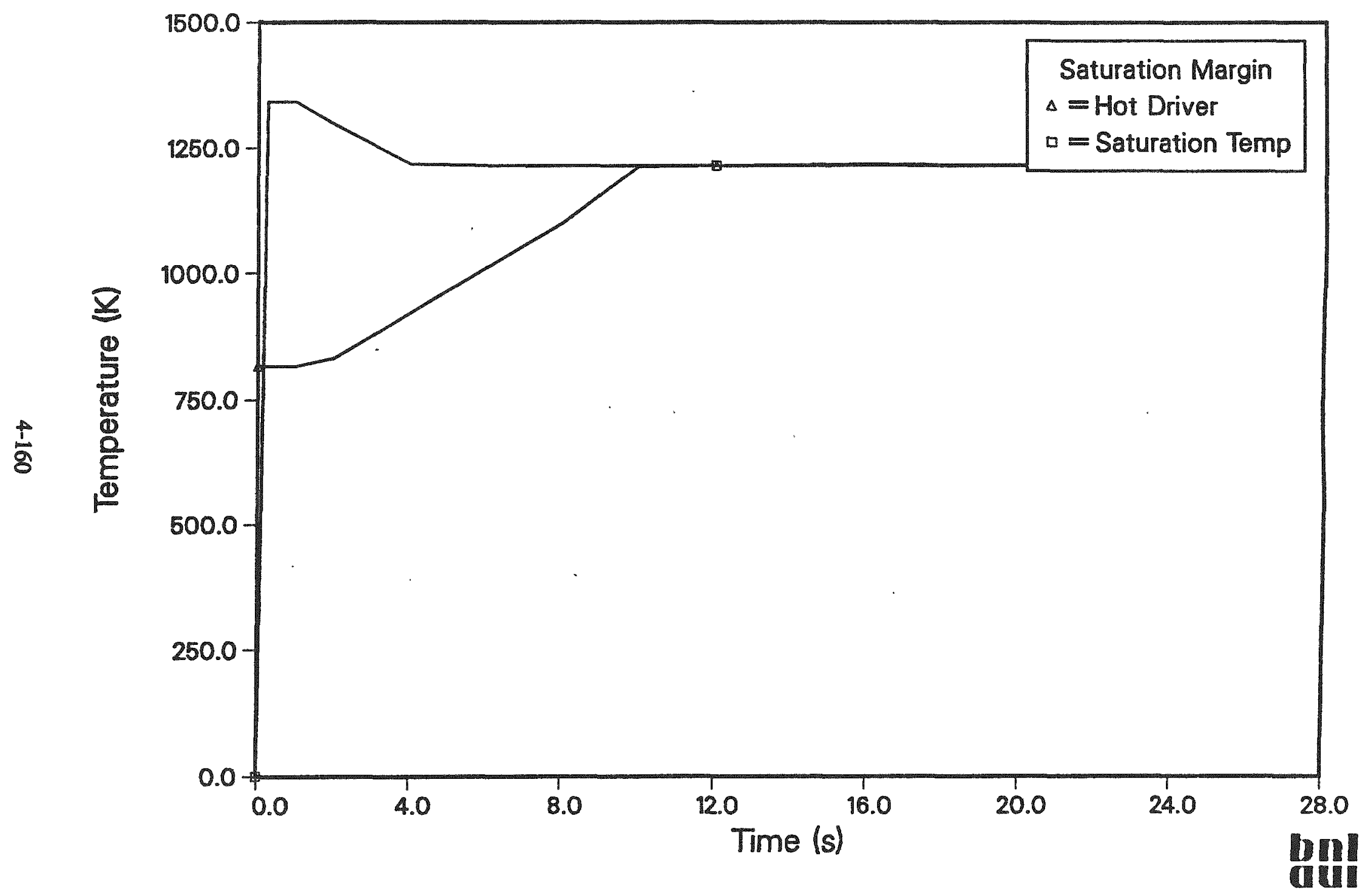

Figure 111. Predicted Margin to Sodium Saturation from SSC for a Total EM Pump Failure. 


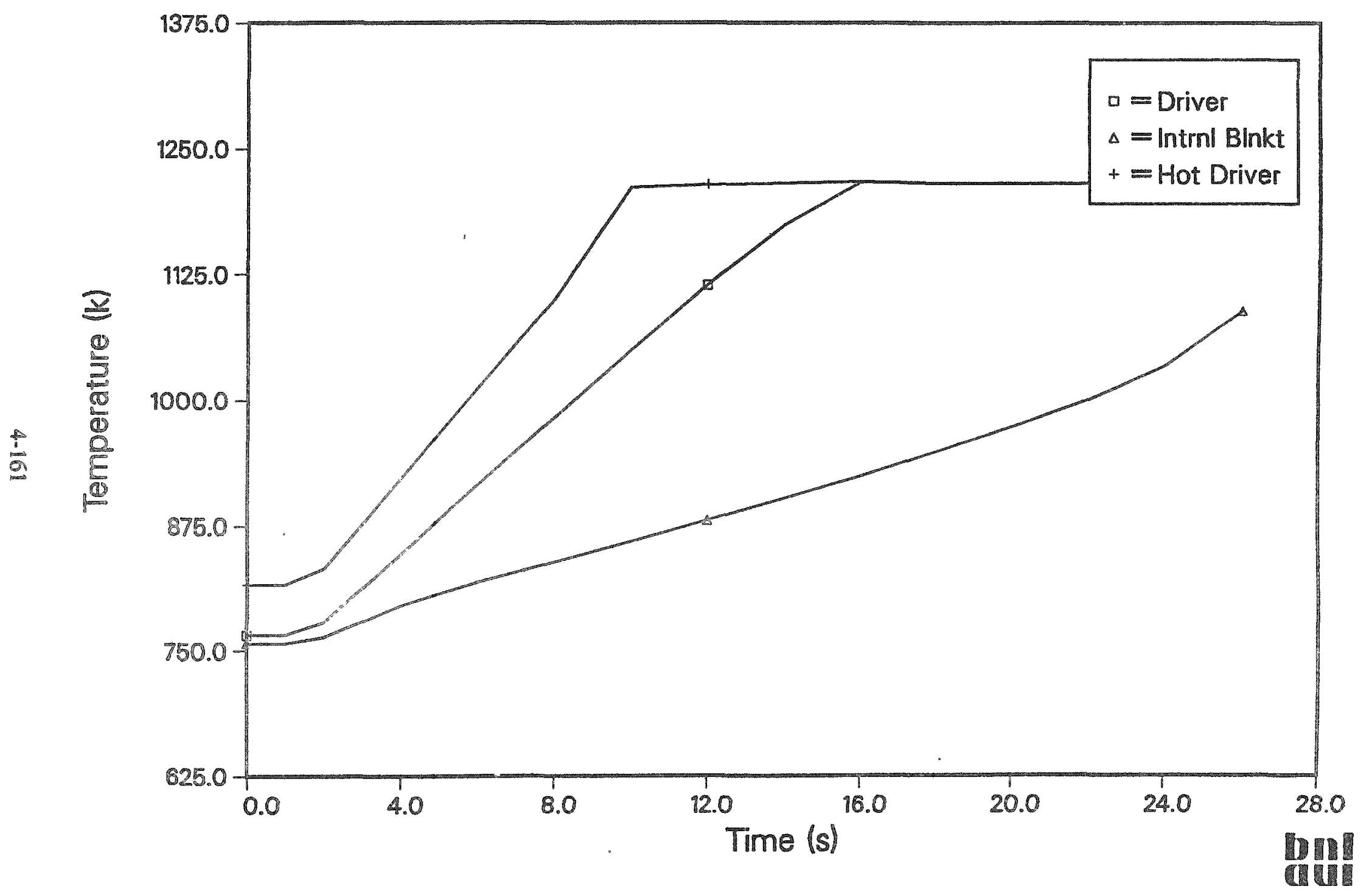

Figure 112. Channel Exit Sodium Temperature from SSC for a Total EM Pump Failure. 
Assuming our prediction of the sodium flow rate through the core (Figure 104) is correct, this is clearly an event that must be avoided. The applicant recognizes the importance of assuring a coastdown and as long as the correct steps are taken, this event should remain to have almost zero likelihood of occurring.

A second way of avoiding such a severe event is to keep the scram reliability high. An analysis of the no-coastdown event was performed using the same BNL codes, but this time with a scram coming 0.8 seconds after the pumps trip. The case was predicted with and without GEMs. In both cases, there is a large margin to sodium boiling, and the peak fuel temperatures are acceptable. Cladding damage would be minor. Thus, it is not essential to get the pump coastdowns if the scram is successful.

\subsection{Cross Comparing ULOF Variations}

Peak temperatures from the four calculations, including GE's (Appendix $G$ of Ref. 1) and three by BNL, are cross compared in Figure 113. The two sets of bars on the left should match up well, as we largely agree on the ULOF on 3 out of 4 pump coastdowns. The discrepancy in the peak fuel temperature traces to GE's prediction that the fuel temperatures start upward before falling off. We can not account for this, as we believe the reactivity feedback from the GEMs brings the power down faster than the fuel can heat up due to lower sodium flow. In this case, as in most, the difference in fuel temperature is not significant and there are substantial safety margins for each calculation. There would be some cladding damage in this case, but it should not be extensive unless the event continues for a long time.

The third set of temperature bars in Figure 113 is a sudden pump stoppage ("seizure") without scram. These peak temperatures are fairly low, and this event does not appear to be threatening.

The fourth set of temperatures is for the exceedingly unlikely instantaneous stoppage of all four pumps without scram. The temperatures shown are taken from the last part of the calculation that SSC could complete.

Overall, the addition of GEMs has helped to substantially improve the response for this category of events. Losing a pump or a pump coastdown no longer appears to be a major concern. However, the last case illustrates that the need for the pump coastdowns is very genuine and that one must always guard against anything that could eliminate all four pumps entirely and simultaneously. Finally, while the GEMs can be very helpful in this respect, there is the additional burden of monitoring the sodium level within the GEMs to ensure their prompt and effective actuation.

\subsubsection{Gas Expansion Modules}

The GEMs were added to provide additional negative reactivity in response to loss-of-pumping events. Previously, it was hoped that the inherent and passive reactivity feedbacks of the reactor would be sufficient for the passive shutdown mechanism to prevent damage in an unprotected loss-of-flow (ULOF) event. New information on the performance of the ternary metal fuel and more careful analysis of the various fuel loading anticipated indicated that additional negative reactivity might be needed in a ULOF event, so the GEMs were added.

The GEM is essentially an empty assembly duct, sealed at the top, open at the bottom, and connected to the core high pressure inlet coolant plenum. When the pumps are operating the trapped gas is compressed into a region above the active core, sodium occupies the portion of the GEM that is adjacent to the fueled region of the core. When the pumps are off, the gas region expands into the core 


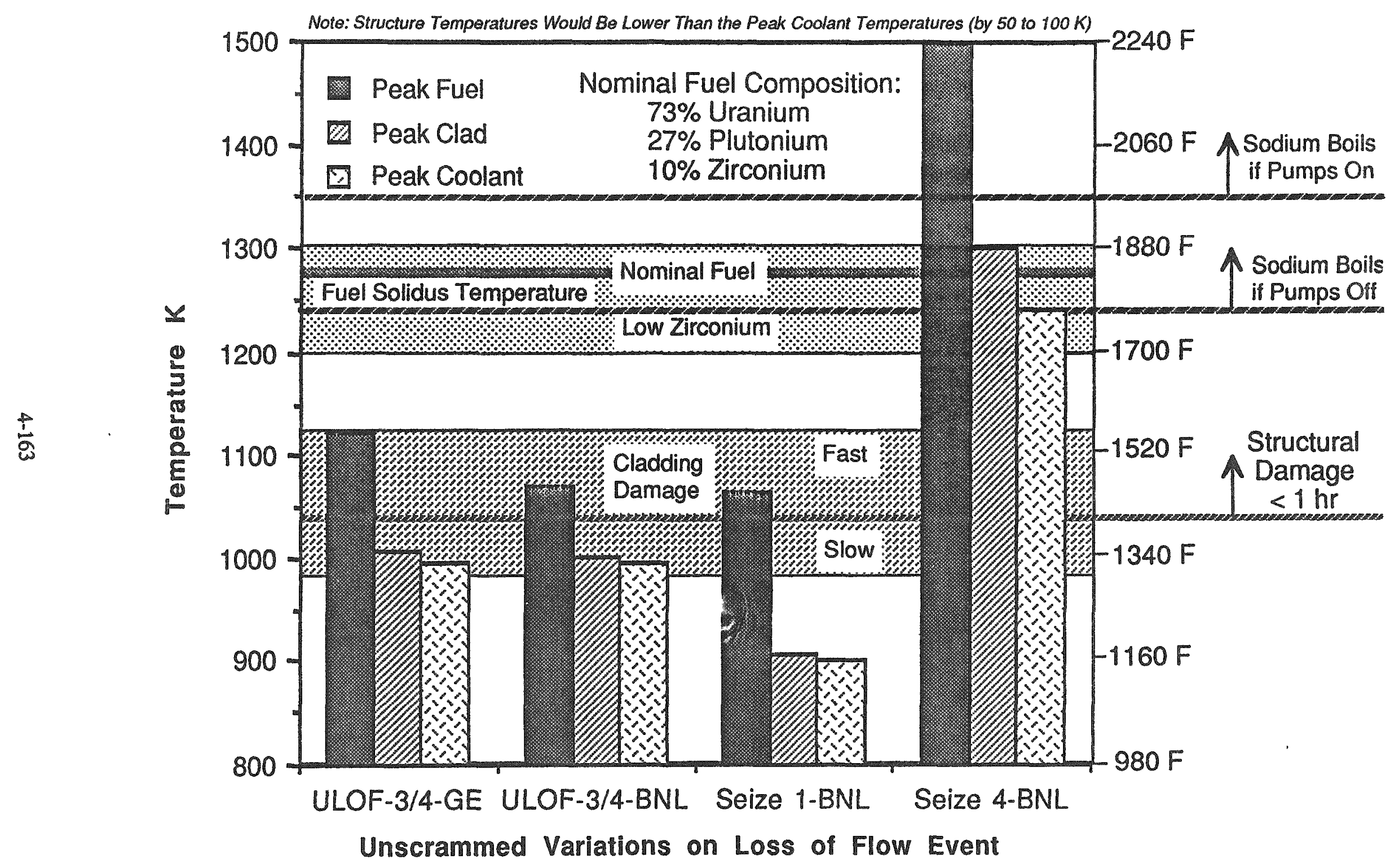

Figure 113. Peak Fuel, Clad, and Sodium Temperatures for Variations on the Unscrammed Loss of Flow Event. 
region and allows more neutrons to escape from the core (reduced scattering back into the reactor). Such devices were successfully tested in FFTF during a series of ULOF tests.

The predicted sodium levels in the GEMs for various (static) conditions are shown in Figure 18 (Section 4.16.3.1.1). For full pumping and flow, such as cases $B$ and $C$ in the figure, the gas space is well above the core. For cases where there is little or no sodium flow, such as case $\mathrm{D}$ and $\mathrm{E}$, the sodium level is near the center of the core. This refueling condition has not been considered in any detail, but most refueling operations are at cold, subcritical conditions so that the impact of any spurious reactivity insertions should be far less threatening than if they occurred at power.

Behavior of the GEMs and their impact under postulated transient conditions are discussed in Section 4.16.3. Their impact is highest for the unscrammed loss-of-flow events, especially during the pump coastdown phase. They appear to be highly effective and helpful during such an event, and even provide extra margin in case the synchronous machines are less effective than predicted, or if one or two might fail entirely.

There are potential safety issues associated with the addition of these devices. First, can they be tested in position on a routine basis? The applicant indicates that they can test the GEMs by varying the pump speeds, while the reactor is subcritical. In principle, we believe such testing could be performed safely and that the results should be fairly accurate, based on the change in subcritical neutron multiplication factors. Second, are they reliable and can deterioration in performance be detected during operation? It appears that the applicant has at least three options for monitoring the GEMs: by using tag gas that can be detected if leaked, by monitoring the neutron flux on the outer (away from the center of the core) side of the GEM duct, and by subcritical testing of the GEM worth during plant outages. Third, could the GEMs inadvertently insert $70 \mathrm{c}$ of reactivity at a crucial time? The applicant claims that it will be nearly impossible to reach full power critical without the pumps being on, and therefore the gas must be compressed into the region above the core under full power conditions. However, this argument is based largely on operating procedures and human factors, so this possibility may turn out to be the major risk from having GEMs in the core.

In summary, the applicant states that they must yet carefully weigh the risks vs. the benefits from having the GEMs in the design. With the GEMs in use, the PRISM response to ULOF events is significantly improved. On the other hand, there may be some risks associated with their utilization, although no major problems have yet been identified.

\subsubsection{Control Rod Stops}

The PRISM Control Rod Stop System is one of the more important safety systems in the current design, as it limits the potential magnitude of UTOP initiators. This importance is due in large part to the expected high reliability of other key safety systems, but also due to the potentially rapid and severe development of reactivity events in LMR cores.

While it's difficult to develop meaningful probabilistic risk numbers for a system such as PRISM, where key safety systems are new, untested, and largely passive, one can make some pretty good guesses as to the weaknesses in the system. For the PRISM design documented in Appendix $G$, the analysis points directly to the unscrammed transient-over-power (UTOP) event. It is a combination of small Doppler feedback (small inertia), zirconium, uranium, and perhaps plutonium migration, and low solidus temperatures that creates the vulnerability for the metal fuel core. The best defense against the UTOP vulnerability is to assure that only small reactivity insertions are possible. In the current PRISM design, this is achieved via the rod stops. 
The current UTOP limit of $40 c$ (including $10 \mathrm{c}$ to cover uncertainties) may be a bit too high, as shown in Sections 4.16.2 and 4.16.3.3.1. However, if this is reduced into the range of $30 \%$ to $35 \mathrm{c}$, then the UTOP predictions may move into an acceptable range. Therefore, it is quite possible the rod stops may be adjusted more frequently so as to limit reactivity insertion to less than 406.

The rod stop system performs an out-motion blocking function, but should have no impact on rod insertion, either forced or gravity driven. The rod stops are moved periodically when the top of the control rods move within some finite distance of the rod stops. The plant control system determines when the rod stops are moved and by how much, but cannot actually move the rod stops without operator permission. The operator can deny permission to move the rod stops, but cannot move the rod stops to an alternate position. Also, all normal rod movement (excluding scram) is temporarily stopped while the stops are adjusted.

GE provides some arguments as to why this approach should be safe and acceptable to the NRC. They state that the data used by the PCS to determine the next move is verified using RPS data (a safety grade system). Also, if the operator does not approve of the proposed relocation, he can prevent the rods movement. Because the burnup swing is negative, failure to move the rod stops would eventually lead to an extremely gradual reactor shutdown (but wouldn't impact on the scram capability).

The rod stop system may provide a viable means of limiting the size of potential UTOP initiators, but there may be room for improvement in a couple of areas. Giving the large non-safety grade PCS control over such an important safety system may not be the best choice. If the operator is going to be able to make a judgement regarding the proposed adjustment he will need reliable instrumentation in the control room and adequate training to be able to make such a judgement. Also, the determination of how far up the rod stops should go must be done using a fairly elaborate calculation based on rod worth curves, burn-up data, and detailed core-physics analysis. On the other hand, movement of all six rods one-half inch is worth slightly less than 10 cents, so the precision of the mechanical relocation does not seem a major concern.

We believe that GE can improve on the rod stop system they currently propose. It is probably not appropriate for their PCS to adjust the rod stops, and it may be preferable for this function to be transferred either to the RPS or another dedicated safety system. If the operators are to have the power to veto a proposed adjustment, they must have a clear and reliable picture of the current conditions. Finally, a simple algorithm could be developed to determine the proposed adjustment in position. Such an algorithm, which might be based on the impact of recent adjustments in control rod position, should be highly reliable.

\section{Key Issues/Concerns in Section 4.1.16}

- Since the postulated UTOP events lead to some localized fuel melting, some policy decisions may be necessary with respect to how much localized fuel melting is acceptable in the ternary metal fuel.

At elevated temperatures, the HT9 cladding begins to interact with the fuel to form a low-melt eutectic. The eutectic depends, in part, on the composition of the fuel in the outer radial zone, which in turn depends on the burnup level and the amount of component migration in the ternary metal fuel. It appears possible for significant eutectic formation to develop about $903 \mathrm{~K}$, which, if confirmed, would indicate some cladding damage during several postulated events. 
- The component migration in the fuel is not fully understood, although additional data is expected over the next year.

- The current postulated UTOP event is 40 cents, and would result in some fuel damage. If the applicant chooses to reduce the maximum UTOP initiator, how will this be accomplished?

- Failure of the pump coastdown devices during ULOF class events could be hazardous. While the failure of more than one of the coastdown devices is expected to be small, one should take precautions to make sure the chances of such multiple failures are indeed very small.

- The GEMs are very helpful for ULOF events, but the applicant needs to provide a means of monitoring the gas space during operations.

- The Control Rod Stops must be adjusted often and with some precision. The procedure has not been defined by the applicant.

\subsection{Station Blackout}

This is an issue that was originally raised by the NRC staff, and we defer to them regarding the applicant's response in Appendix G. We add a few comments here just to note our perspective on station blackout.

The PRISM reactor was designed to passively accommodate loss of power events, and its performance under station blackout conditions should be excellent. The requirement for safety grade (class 1E) power is low, approximately 60 kilowatts for a nine module plant, and can be supplied entirely from batteries. We believe that the lack of Class $1 \mathrm{E}$ diesel generators may actually be an advantage of this design, as one does not need to worry about the probability of a diesel generator starting up on demand.

Key Issues/Concerns in Section 4.17

- None - PRISM was designed to accommodate this event passively.

\subsection{Risk Assessment}

The original PRA included with the PRISM PSID was enlightening in several respects. It provided some insights regarding the design, especially with respect to the relative importance of some of the key safety systems. The big challenge was in assigning meaningful numbers to some of the system failure rates, as many of these systems were new and different from anything previously evaluated. In several cases there was no existing data base from which to extrapolate. Perhaps the greatest challenge was the RVACS, which was the only safety grade decay heat removal system and had no apparent failure mode other than very large earthquakes and extremely thorough acts of sabotage. In addition, key data were missing regarding the behavior of the metal fuel during severe accidents. As a result, the original PRA contained several very large uncertainties. The SER indicated that the initial PRA was interesting and represented a reasonably good effort at tackling a very difficult task. However, the outcome of the PRA was dominated by uncertainties, and it was felt that to draw firm conclusions from the PRA required some degree of caution.

The applicant's response, provided in Appendix $G$, has both encouraging and discouraging elements. They point out several instances where design changes strengthen their cases regarding the 
safety of PRISM, and in many cases we would concur. In addition, the applicant also attempted to improve on the PRA, and to use sensitivity studies to argue that some of the areas with a lot of uncertainties are not really that important. This should be viewed with caution, however, as some of the failure rates assumed by the applicant contain enough uncertainty to distort the sensitivity study itself.

\subsubsection{Impact of Design Changes}

Several design changes are cited in Section G.4.18.2, and most appear to improve the PRISM safety picture. Key changes are discussed in the sections that follow.

\subsubsection{Seismic Isolation of the Synchronous Machines}

This appears to be a significant improvement, as these machines must provide a highly reliable "coastdown" for the EM pumps (which have no inherent coastdown). The previous arrangement had the cables between the EM pumps and the corresponding synchronous machines crossing between zones that were seismically isolated and those that were not. This appeared to be a significant safety problem, and its elimination should reduce the risk.

\subsubsection{Strengthening Buildings and Enclosures}

The refueling enclosure, steam generator building, and the control building have been tornado hardened. In addition, the refueling enclosure has been upgraded to Seismic Category I, and the steam generator building and control building have been upgraded to Seismic Category II. Clearly, these are improvements that are potentially important to safety, and should reduce the risk due to external events. In particular, providing better protection for the operator is thought to be a significant improvement.

\subsubsection{Gas Expansion Modules (GEMs)}

It is not clear whether the addition of GEMs will ultimately be considered a plus or a minus for PRISM. They will add significant negative reactivity (leakage) when the pumps slow or stop, and are therefore helpful to the survival of postulated unscrammed loss-of-flow events (ULOFs). They also insert reactivity when the pumps are started. However, for the previous design, GE and ANL believed the passive shutdown could work sufficiently well without GEMs. Newer fuel and design information indicated that the GEMs might be needed in order to survive a ULOF, and the GEMs were added to the design. As with any "gadget", there is always the possibility that they will not always perform as expected, so their addition to PRISM might trigger some new accident initiators and/or complications. It is even possible that the GEMs might actually increase the overall risk, even though they are currently required for the passive shutdown to work well for the ULOF event.

\subsubsection{Ulimate Shutdown System (USS)}

The addition of the USS should reduce the risk, particularly regarding unscrammed events. Of course, there are some questions regarding performance of the USS, but we are hopeful that these can be resolved without much difficulty. Our previously stated concerns regarding the adequacy of the passive shutdown mechanism as a "second and diverse means of reactor shutdown" are now largely resolved, particularly for the long, slow events that allow time for the USS to shut the reactor down (a minute or longer). 


\subsubsection{The Control Rod Stops}

Previously, claims by GE and ANL that the burnup reactivity swing could be limited to a few cents were regarded skeptically, although we were willing to perform our UTOP analyses based on the claim. After some more data were added and more detailed analyses were performed, the designers determined that a burnup reactivity swing in excess of $\$ 1$ was to be anticipated. As the passive shutdown is adequate for only a 30 to 40 reactivity insertion, the applicant was forced to add the rod stops, which can be adjusted occasionally to assure that the maximum UTOP initiator is small enough to allow the passive response to function properly. The need to use rod stops to prevent a larger UTOP increases risk, although their incorporation in the design at this early stage is probably prudent.

\subsubsection{Designing to Accommodate HCDAs}

This is potentially an important reducer of risk, as this class of accidents could have rapid and possibly severe consequences. If the PRISM system could indeed withstand a worst-case HCDA and maintain structural integrity, the likelihood of early fatalities from any postulated accidents would be very small. Our primary reservation here is that the data for "prefailure" (before the reactor fails) extrusion of the metal fuel is very limited, so the size of potential HCDAs is not well known. In addition, there now appears to be the possibility of the solidus temperature being exceeded in the center of the core but not near the top of the core, which could preclude the extrusion from occurring as is required to terminate an HCDA event. However, the analyses discussed in Reference 9 suggests the energy release in a metal fuel HCDA would be significantly less than the $500 \mathrm{MJ}$ currently assumed by the applicant, suggesting the PRISM system could survive a large HCDA event.

\subsubsection{Designing to Accommodate Core Meltdown}

GE's modifications to the lower core support structure may make safe accommodation of a whole core meltdown within the lower region of the vessel more likely, and thus decrease risk somewhat. However, there appear to be shortcomings. For one thing, the core must move from the normal configuration and transition to the support region without achieving recriticality. Also, the cooling mode for the vessel would be via the RVACS, primarily. However, most scenarios resulting in core melt involve failure of the RVACS, so it seems unlikely that the RVACS would be available to cool the molten core. Therefore, this design modification may reduce the risk somewhat, but the improvement may not be quite as significant as it first appears to be.

\subsubsection{The Containment Dome}

The addition of a containment dome makes PRISM more closely resemble a conventional U.S. power reactor, but the overall reduction in risk is probably far less than one gets from a LWR containment. This is due primarily to the capability to retain fission products in either the molten fuel or the sodium pool, which should reduce the type and amount of radionuclides reaching the containment atmosphere during an accident. However, the dome is helpful for mitigating sodium fires and delaying the release of fission products in a worst case accident.

\subsubsection{Sensitivity Studies}

The original PRISM PRA contained some failure probabilities that were difficult to justify. For example, failure rates for the scram system in the range of 1 in a billion, and for RVACS in the range of 1 in a trillion, were inappropriate. These are well designed systems that should have low failure rates, but no system is completely fool-proof. 
Our concerns regarding these assumed failure rates were discussed with the applicant on multiple occasions. We were encouraged when their PRA experts indicated their intent to revise the PRISM PRA. However, the materials included in the latter part of Appendix G.4.18 indicate that there remain some philosophical differences. In particular, some of the extremely low assumed failure rates may have distorted the basis for the sensitivity studies.

One can develop a fairly simple summary PRA based upon three major factors: reactor shutdown, shutdown heat removal, and retention of fission products. The two systems that stand out are the scram system and the RVACS. Of slightly less importance are the passive ("inherent") reactor shutdown, the USS, the alternate heat removal systems (normal and ACS), and the tendency of the fission products to remain in either the molten fuel or the sodium pool. To some degree, statements by the applicant are fairly consistent with these estimates. However, with such large uncertainties regarding the failure probabilities of key systems, it could be a mistake to focus too closely on the "bottom-line" risk estimates.

Key Issues/Concerns in Section 4.18

- The reliabilities for passive systems, such as RVACS, are not known and could vary by several orders of magnitude depending on the evaluator.

- For such new and radically different designs, the PRA will include many numbers that can not be adequately supported by test data or operational experience. Estimates of core damage frequencies and large release probabilities could be off by two or three orders of magnitude. Further, even sensitivity studies could contain errors large enough to distort the results.

\subsection{Mitigation of Severe Core Accidents}

While not really in response to any single safety and/or licensing issue in the SER [Ref. 2], this section addresses a broader concern that was expressed in several meetings and alluded to in several places in the SER. Prior to receiving this feedback, GE along with DOE and ANL, felt that by providing a strong case in support of their contention that severe accidents can be prevented in a metal-fueled PRISM reactor, they could avoid devoting scarce resources to accident mitigation. However, there were two problems with this approach: uncertainties and recent history. With respect to the uncertainties, the metal fuel seems to have some genuine safety advantages, but ANL's knowledge of the fuel is largely empirical, i.e., they know how it behaves because they have observed it in experiments. The problem is that there is little data available on the $26 \%$ Pu ternary metal fuel designated for PRISM, and the data on lesser-Pu ternary fuel is somewhat different than they expected based on the uranium metal fuel. With respect to recent history, based on a few complicated accidents that have developed in the last ten to twelve years, including two that were quite severe, the nuclear safety community has become more committed to providing accident mitigation capabilities, in order to respond to "what if..." questions.

Upon receiving considerable feedback regarding the desirability of providing a strong accident mitigation capability, GE, ANL, and DOE are now making an effort to strengthen the design in this regard. They have added a containment dome to PRISM, and are working to show some increased capability for accident mitigation. However, some key portions of the required data base have never been developed, and can not be developed quickly.

The bulk of Appendix G.4.19 covers two analytical efforts that attempt to use simple engineering principals to show that two worst case scenarios may not be as damaging as one might assume. This 
work reflects some degree of ingenuity, and provides some helpful reassurance, although there are currently no guarantees that the results are correct. We consider these analyses in the order they appear in Appendix $\mathrm{G}$.

\subsubsection{Impact of HCDA on PRISM Vessel and Structures}

It is assumed that the analysis that was performed to assess the magnitude of possible power excursions in FFTF, which uses oxide fuel, could be used to estimate an upper bound on the maximum excursion that could develop in PRISM. The ramp rates, which were in excess of $\$ 100 /$ second, and the energy release, which ran a few hundred megajoules, are quite high and could be considered conservative-at least for the FFTF oxide core. The analysis presented in Reference 9 suggests that the energy release from a metal core should be smaller than that from an oxide core, for reactivity insertions above $\$ 100 /$ second. Thus, it seems highly probable the $500 \mathrm{MJ}$ from the FFTF case would bound a PRISM HCDA.

The Appendix G analysis indicates that the PRISM vessel and structures could likely absorb the large energy release predicted for FFTF. Our independent estimates tend to contirm this, i.e., the large HCDA postulated for FFTF could probably be accommodated in PRISM without large scale failures resulting. However, the structural calculations don't include any radiation-induced embrittlement that might develop. One example would be the core barrel, which will see increased fluence in the sectors directly out from the $3 \mathrm{GEMs}$, and could undergo embrittlement as a result.

\section{Conclusions/Recommendations}

Since the projected FFTF HCDA energy release estimate may well bound that expected for PRISM, it is entirely possible PRISM could survive such an event without catastrophic failure of the vessels or key structures.

\subsubsection{Analysis of Molten Core on Support Plate}

Because a sodium cooled reactor is not designed to operate in its most reactive configuration, any fuel relocation could well result in a significantly supercritical mass. As a result, a molten metal fuel core would be very difficult to predict. It is possible that the fuel could gradually relocate and accumulate down on the core support plate, and that is the condition that GE/ANL chose to analyze.

The analysts considered four scenarios, including relocation of the active fuel alone, the active fuel and its cladding, the fuel and blanket materials (with cladding), and virtually the entire core. They do not specifically mention any sodium, although some experiments indicate that molten metal fuel is very porous and would certainly contain a considerable amount of sodium. They then estimated the infinite neutron multiplier, $\mathrm{k}$-infinity, for each of the four compositions. The first two composites, i.e., materials coming only from the active fuel region, have $\mathrm{k}$-infinities in the range of 1.9 . While this is quite high, there is a great deal of fissile plutonium in the PRISM active fuel, and a $\mathrm{k}$-infinity even higher than 1.9 may be possible. The remainder of the analysis involves geometric considerations, assuming the melt spreads evenly on the core support plate, and involves estimating critical heights that would have to be attained before $k$-effective reached 1.0. It turns out that the critical height can not be reached for each of the four composites, due largely to the degree of spreading on the support plate. GE also points out that if there existed a real hazard of achieving a critical mass on the plate that they could add neutron poisons to the region-just in case. 
In response to questions regarding the transition phase, the applicant analyzed a case where the fuel assemblies melted and the fuel then accumulated in the lower shields. Using some worst case assumptions, the applicant predicted a maximum $k$-effective of 1.28 . In response, the applicant showed that by using some natural $\mathrm{B}_{4} \mathrm{C}$ in the region, the maximum $\mathrm{k}$-effective could be pushed sub-critical.

Having determined that the mass lying on the core support would most likely be subcritical, GE proceeded to analyze the long term heat removal and materials damage. Presumably they have assumed porous fuel with sodium in the pores, although this is not specifically stated. GE does include some analysis of the damage to the core support structure due to the prolonged exposure to the fuel, and states that they had to add a 2-inch backup plate to compensate.

There is an apparent flaw in this analysis. A key factor in some of the scenarios that could result in core melts is failure of RVACS to remove the after-heat. However, the applicant is assuming that when the molten core is settled on the core support structure the heat is going to be removed via the RVACS (this is implied by Figure G.4.19-11, anyway). This points to a possible weakness in the PRISM containment design, i.e., if the cooling systems that are there to prevent core melt fail, it may not be possible to cool the containment vessel, so the containment may fail also.

Key Issues/Concerns in Section 4.19

- The applicant's arguments that the HCDA energy release calculated for FFTF bounds the HCDA release for PRISM has merit. It appears possible that a large HCDA could be accommodated within the PRISM vessel and head region.

- The applicant' analysis showing subcriticality of a molten core when distributed on the belowcore support plate is useful. However, there is great uncertainty involved with the process of relocating the fuel from the original core to the support plate. 


\section{SUMMARY AND CONCLUSIOUS}

The recent design revisions, made largely in response to NRC concerns, have been quite significant and have changed some of our perceptions regarding the safety of this design. The increase in reactor power (for economic reasons) may have reduced some safety margins, and the switch to a single-wall-tube helical coil stean generator may increase the likelihood of a tube leak and a sodiumwater reaction (note: this steam generator design also has good accident mitigation capabilities). Addition of the Ultimate Shutdown System (USS) and the containment dome are believed to be significant safety improvements. Modifications to the below-core structure, the seismic design (especially regarding the placements of the synchronous machines), and the IHTS Auxiliary Cooling Systen (ACS) are also considered to be substantial improvements. Some changes were also required to compensate for a more current assessment of the ternary metal fuel. These changes include reactor-redesign, and the addition of the GEMS and the control rod stop system. Our current assessment is that these three changes were necessary, and that most of the apparent shortcomings in these revisions can be reduced with time.

Our overall evaluation of these changes is that the PRISM systen design has been improved, but that a more detailed evaluation of the ternary metal fuel has revealed some significant problems. It must be recognized that the ANL experience with the high $\mathrm{Pu}(26.5 \%)$ ternary metal fuel has been quite limited, and that some problems should have heen expected. Given time, it seems likely that ANL will resolve some of the current questions about plutonium migration and low eutectic remperatures.

The research and development program outlined by GE covers many of the higher priority needs, and includes some indications that there will be increased emphasis on examining factors important to accident mitigation. It is clear that most of the R\&D support will be in the metal fuels area, particularly a fuel cycle facility (HFEF/South) and further testing in EBR-II. Part of this work will likely examine the impact of factoring minor actinides into the ternary fuel, which should take place if LWR spent fuel is to be used to produce the initial fuel loading for PRISM. If the only minor actinide feed for PRISM is that which comes along with LWR spent fuel plutonium, the impact on core physics parameters may well be acceptable. (However, a special purpose machine having more minor actinides than uranium and plutonium would have some significant reactivity problems and would bear little resemblance to PRISM).

Although GE did not discuss their planned R\&D into key severe accident issues, such as rapid axial expulsion and the behavior of molten metal fuel in sodium puols, it is clear that they, along with DOE and ANL, plan to increase the efforts in these areas. We recoguize the difficulty in developing such data, and would not expect to see major results in the near rerm.

The bulk of GE's Appendix G (Reference 1) is addressed 1019 "Safety Issues", as interpreted by GE from the draft SER (Reference 2). Our evaluation of these responses follow.

\section{Containment}

The PRISM reactor system is very different from light water reactors (LWRs), so one has quite different design objectives. With respect to a possible sodiun fre event, analysis indicates the current containment design should properly contain a worst-case sodium pool fire. Our principal concem regarding the PRISM containment is that key data for metal fuel behavior during severe accident is unavailable, so it is very difficult to characterize the potential challenges to the containment. 


\section{The Shutdown System}

Because of the large positive sodium reactivity worth, and despite the passive reactor shutdown characteristic, the reactor shutdown system is one of the most important safety systems in PRISM. The main shutdown system, based on six mostly independent control rod sub-systems, appears to be highly reliable and redundant. However, the recent addition of the Ultimate Shutdown System adds an alternate shutdown system and thereby largely addresses our previous concern. With respect to the Control Rod Stop System, we recognize the need for such a system. However, the applicant may have to do more design development work before one can fully assess this system.

\section{0-Year Plant Life}

This is an issue that is addressed primarily by the NRC staff. We do not know of any technical reasons that might preclude a 60 -year life, assuming that key components are replaced by the end of their useful lifetimes (e.g., the pumps).

\section{Seismic Isolators}

The PRISM seismic isolators are relatively simple devices which provide a significant degree of horizontal isolation, and are especially helpful for the tall and narrow PRISM system. Our main concern is with respect to data and experience, which will undoubtedly come with time.

\section{Sodium Void}

GE and ANL are making progress in trying to address our concerns regarding the large positive sodium void reactivity worth. Clearly, no one wants this design characteristic, but the options for eliminating it are limited and have undesirable impacts on other design characteristics. GE's stated response has three components. First, the chances of the sodium boiling are very small (we concur). Second, if the sodium boils the resultant HCDA could probably be absorbed without catastrophic results (could be true). Third, their most viable options for reducing the sodium void worth, using the metal fuel, is to "spoil" the geometry so as to increase neutron leakage and the "increased leakage" component of the sodium voiding reactivity feedback. Unfortunately, this increases the burnup reactivity swing, and therefore the potential size of the UTOP initiator.

\section{Flow Blockages}

The most credible scenario for a flow blockage seems to be through a manufacturing error, e.g., when someone fails to drill the holes. This should be spotted before the assembly is loaded, and should be quite obvious at low power testing - assuming the assembly is actually loaded.

\section{Electromagnetic (EM) Pumps}

The EM pumps are complex and lack an inherent coastdown mechanism, which is a disadvantage with respect to PRISM safety. However, these pumps apparently provide some real advantages in the operation of PRISM, at least in the opinion of the applicant. The addition of GEMs to the reactor system design has reduced our concerns significantly, as a loss in pumping will result in a quick reduction in reactivity and therefore reactor power. On balance, we are more inclined to accept the EM pumps than we were previously, but would be more comfortable with a pump which has an inherent inertia. 
By switching from the straight double-tube-wall steam generator (like EBR-II) to the helical-coil single-tube-wall steam generator, GE has probably increased the likelihood of a tube rupture but has also improved the capability to deal with such an event. The main objective is to prevent any damage to the IHX, and we believe the revised SWRPRS is very likely to prevent any such damage, based on both an active (isolation valves) and a passive (rupture disks plus carefully chosen piping elevations) sub-system.

\section{Reactor Vessel Auxiliary Cooling System (RVACS)}

RVACS is one of the most important safety systems in the PRISM design and is very difficult to defeat. Our calculations have consistently confirmed those submitted by the applicant, and we feel that this is an excellent decay heat removal system. It is difficult to develop any reasonable failure probabilities for this system, and we suspect a massive seismic event may be the dominant contribution. There is a very subtle failure mode that may eventually prevail for this and other passive systems. This is based on system degradation coupled with a primary failure, e.g., the reactor vessel leaks and the heat transfer surfaces are fouled. Of course, this type of failure mode may be very low, say around once in a million reactor years, but this could be the dominant mode for a system that is very highly failure resistant.

\section{Control Room}

In comparison to the current generation of nuclear power plants, the PRISM safety systems are largely passive and require almost no operator actions. GE feels that the amount of information provided to the operator and the degree of protection provided for the operator can be decreased somewhat. However, there are a few isolated cases where the operator could be very important, so it is difficult to accept GE's position. Our current perception of the PRISM system safety is that the operator must have an assured capability to scram the system and enough instrumentation to recognize the need to scram.

\section{Emergency Preparedness}

Because of the various safety characteristics of the PRISM design, GE feels that detailed emergency evacuation planning and the testing of that plan is an unnecessary burden. It is true that severe accidents appear to be very unlikely, according to current PRA estimates. On the other hand, the accident mitigation capabilities are very limited, and rely largely on characteristics such as rapid fuel extrusion during HCDAs and fission production retention during core melts. Unfortunately, the supporting data bases in these "severe accident" areas are very limited. Until these phenomena are better understood and documented, it will be difficult to assess the need for detailed emergency evacuation planning.

\section{Role of Operator}

It is apparent that the PRISM operators would play a greatly diminished safety role in comparison to those currently operating light water reactors (LWRs). This is a major advantage for this design. However, they will still have some safety responsibilities, including activating the USS if the primary scram system fails and identifying any manufacturing defects in flow assemblies before going to full power. Therefore, while the PRISM operators should not be as crucial to safety as those operating LWRs, there will be cases where they must fulfill a safety function. 


\section{Muli-Module Control}

A nine-module PRISM plant should be controlled by a state-of-the-art digital control systen. The technology is available or can be readily developed, and we do not see this as an excessively challenging endeavor. However, the size and conplexily of such a system would make quality assurance and/or independent assessment a very formidable task. The more practical approach would be to determine that the reactor is sufficiently well protected by the Reactor Protection System (RPS) and by the passive response characteristics, such that errors generated by the control system would not be damaging. This is not likely to be quite as dificult as it sounds, as we are already assuming that several limiting cases will occur, and the PRISM system response has generally been within acceptable limits.

\section{Security}

This issue has been handled primarily by the MRC staff, and we have already provided input regarding this issue.

\section{Prototype Tests}

We consider the prototype resting of the first PRISM module to be essential, particularly with respect to the passive shurdom response. Early and committed participation by the $\mathbb{N R C}$ staff should be an essential component of a successful test program.

GE's desire 10 substipute an air-dump heat exchanger for the steam generator and sodium-water reaction protection relief system makes sense economically. The prototype would be much cheaper without these large and complex systens, and the impact on the test program would be very modest. However, some testing of these systens would be necessary, perhaps using some separate facilities.

\section{Safery Analyses}

Several postular ed events were analyzed using independent codes, such as SSC and MNET. In most cases, we could duplicate GE's calculations, and we feel we understand most of the differences in our predictions.

Generally speaking, the PRISM RVACS has consistently stood up to close scrutiny, and we believe this is a very robust system. It is difficul to adequately factor this system into a PRA, because most failure modes are barely credible. We suspect the most plausible failure mode may be a single failure (blockage of one duct or a leak in the reactor vessel, for example) in combination with previously unnoticed degradation in some key performance parameters. While this combination may be unlikely, it may be more likely than either a double vessel failure or the complete blockage of all four air ducts.

The situation regarding the passive reactor shutdown is far more complex, especially since the flow of data for ternary metal fuel has only recently begun. We are hopeful that ANL will eventually obtain the performance that is required, even should a few adjustments in the fuel composition or density be required. However, we do have to express our reservations in this regard, as the ternary metal fuel behavior appears to be very complex and there is evidence of some undesirable redistribution of the uranium, zirconium, and perhaps the plutonium components.

The greatest challenge, at the monen, appears to be the unprotected transient-over-power (UTOP) event, which boosts luel centerline temperatures. We believe that a 40 initiator may be too large, given our current knowledge of the ternary fuel, and think that GE may need to adjust the rod 
stops more frequently so as to reduce the largest TOP initiator into the $30 \mathrm{c}$ range. GE clearly has some options in this area, so it seems wise to withhold judgement until GE proposes design changes to compensate.

Because of the GEMs, the passive shutdown now appears to work much better for the ULOF events. As long as there is at least a partial coastdown provided by the synchronous machines, we think PRISM would survive the ULOF category events.

The PRISM passive shutdown has always appeared to perform well for the ULOHS events, and nothing has changed significantly with the revised design. If there are problems here, they will develop only if the event continues for a long time, and the addition of the USS makes this very unlikely.

The main problem with the non-TOP unscrammed events is at the fuel-cladding interface. ANL seems confident that the rate of eutectic formation at the temperatures experienced during the unscrammed events is very slow, so minimal damage is to be expected. However, the behavior of the ternary metal fuel is very complex, and further experimental data will be needed before one can conclude that the cladding damage is minimal.

\section{Station Blackout}

The PRISM system was designed to responsd passively to a station blackout event, and we believe such an event would pose little threat to the PRISM reactor.

\section{Risk Assessment}

Because of the assorted passive systems, which are very unlikely to fail, it is very difficult to develop a credible PRA for PRISM. We believe the applicant's PRA experts are trying to accurately assess the PRISM system, but we caution restraint in the interpretation of results from the PRA. Further, the severe accident portion of the PRA is weakened by a lack of experimental data in key areas, such as HCDAs and molten metal fuel.

\section{Mitigation of Severe Core Accidents}

Substantial gaps in the data base make analysis of severe accidents with metal fuel difficult. Having recognized the need to provide some evaluation of the PRISM system response to postulated coremelt and HCDA events, GE has provided some interesting "engineering judgement" as to the most likely outcome of such events. In general, most of these arguments are thought to be plausible, and it seems likely that the netal fuel would probably behave at least as well as oxide fuel under the postulated conditions.

In conclusion, many of the PRISM reactor system changes must be considered improvements, and some of the previous safety concerns have been addressed. The increase in the reactor power production may have reduced some safety margins, although in most cases the margins are still substantial. Ironically, the principal safety issue remaining is the performance of the ternary metal fuel. Certainly there are some clear advantages to using the metal fuel, and ANL seems very confident that any remaining technical problems can be resolved. Until more work has been completed on the ternary metal fuel, one can only say that the metal fuel has the potential to be an excellent fuel in a liquid-metal cooled reactor. 


\section{REFERENCES}

1. Gyorey, G. L., Pedersen, D. R., and Rosen, S., "Safety Aspects of the U.S. Advanced Liquid Metal Cooled Reactor Program," Proceedings of the 1990 International Fast Reactor Safety Meeting, August 12-16, 1990, Snowbird, Utah.

2. Landry, R., King, T. L., Wilson, J. N., "Draft Preapplication Safety Evaluated Report for Power Reactor Inherently Safe Module Liquid Metal Reactor," Nuclear Regulatory Commission Report, NUREG-1368, September 1989.

3. Van Tuyle, G. J., Slovik, G. C., Chan, B. C., Kennett, R. J., Cheng, H. S., Kroeger, P. G., "Summary of Advanced LMR Evaluations - PRISM and SAFR," Brookhaven National laboratory Report NUREG/CR-5364, BNL-NUREG-52197, October 1989.

4. Heusener, G. et al, "The CABRI-Programmes-Motivations and Achievements," Proceedings of the 1990 International Fast Reactor Safety Meeting, Snowbird, Utah, August 1990.

5. Murata, K. K., et al, "User's Manual for CONTAIN 1.1 - A Computer Code for Severe Nuclear Reactor Accident Containment Analysis," SAND 87-2309, Sandia National Laboratories, Albuquerque, New Mexico.

6. Madni, I. K., et al, "A Simplified Model for Calculating Early Off-Site Consequences from Nuclear Reactor Accidents," Brookhaven National Laboratory Report BNL-NUREG-52153, July 1988.

7. Scholtyssek, W. and Jacobs, G., "Validation of the CONTAIN Code and Application to Accident Consequence Studies in a Large Fast Breeder Reactor," Proceedings of the 1990 International Fast Reactor Safety Meeting, Aug. 12-16, 1990, Snowbird, Utah.

8. International Atomic Energy Agency, "Status of Liquid Metal Cooled Fast Breeder Reactors," Technical Report No. 246, page 448, Vienna, Austria, 1985.

9. Om Pal Singh, et al, "Energetics of a Hypothetical Core Disruptive Accident for Different Fuels for a Medium Sized Reactor", Proceeding of the International Conference on Fast Reactors and Related Fuel Cycles, October 28 - November 1, 1991, Kyoto, Japan

10. Cahalan, J., et al, "Performance of Metal and Oxide Fuels During Accidents in a Large Liquid Metal Cooled Reactor," Proceedings of the 1990 International Fast Reactor Safety Meeting, Aug. 12-16, 1990, Snowbird, Utah.

11. Royl, R., et al, "Influence of Metal and Oxide Fuel Behavior on the ULOF Accident in 3500 MWt Heterogeneous LMR Cores and Comparison with Other Large Cores," Proceedings of the 1990 International Fast Reactor Safety Meeting, Aug. 12-16, 1990, Snowbird, Utah.

12. Dobbin, K. D., et al., "Sodium Void Reactivity Comparison for Advanced Liquid-Metal Reactor Fuels," Transactions of the American Nuclear Society, Vol. 63, June 1991, Orlando, FL, pp. 319-320. 
13. Matveev, V. I., et al, "Physical Grounds for Further Improvement of Fast Sodium Power Reactor Safety, " Proceedings of the 1990 International Fast Reactor Safety Meeting, Aug. 12-16, 1990, Snowbird, Utah.

14. Balz, W., et al, "Core Design and Safety Aspects of Large LMFBRs with Minor Actinide Recycling," Proceedings of the 1990 International Fast Reactor Safety Meeting, Aug. 12-16, 1990, Snowbird, Utah.

15. Prunier, C. et al, "European Collaboration on Mixed Nitride Fuel, "Transactions of the American Nuclear Society, Volume 62, page 244, Washington, D.C., November 1990.

16. Bardelle, P, and Prunier, C., "Synthesis, Sintering, and Characterization of Uranium-Plutonium Mononitride," Transactions of the American Nuclear Society, Volume 62, pages 244-245, Washington, District of Columbia, November 1990.

17. Ethridge, J. L., et al, "Fast Flux Test Facility Core System," Transactions of the American Nuclear Society, Volume 62, pages 255-256, Washington, District of Columbia, November 1990.

18. Leggett III, W. D., and Leggett, R. D., "A Decade of Progress in Fast Reactor Fuel," Transactions of the American Nuclear Society, Volume 62, pages 74-76, Washington, District of Columbia, November 1990.

19. Van Tuyle, G. J., et al, "MINET Code Documentation," Brookhaven National Laboratory Report, NUREG/CR-3668, BNL-NUREG-51742, Completed February 1984, Published December 1989.

20. Van Tuyle, G. J., "A Momentum Integral Network Method for Thermal-Hydraulic Systems Analysis," Nuclear Engineering and Design.91, January 1986, pages 17-28, North-Holland, Amsterdam.

21. Guppy, J. G., al, "Super System Code (SSC, Rev. 0) An Advanced Thermohydraulic Simulation Code for Transients in LMRBRs," NUREG/CR-3169, BNL-NUREG-51659, April 1983.

22. Van Tuyle, G. J., et al, "Analyses of Unscrammed Events Postulated for the PRISM Design," Nuclear Technology 21, August 1990, pages 165-184, also: Brookhaven National Laboratory Report BNL-NUREG 42762. 